\title{
WestVirginiaUniversity
}

THE RESEARCH REPOSITORY @ WVU

Graduate Theses, Dissertations, and Problem Reports

2010

\section{Laboratory and Real-World Measurement of Diesel Particulate Matter}

Yuebin Wu

West Virginia University

Follow this and additional works at: https://researchrepository.wvu.edu/etd

\section{Recommended Citation}

Wu, Yuebin, "Laboratory and Real-World Measurement of Diesel Particulate Matter" (2010). Graduate Theses, Dissertations, and Problem Reports. 3013.

https://researchrepository.wvu.edu/etd/3013

This Dissertation is protected by copyright and/or related rights. It has been brought to you by the The Research Repository @ WVU with permission from the rights-holder(s). You are free to use this Dissertation in any way that is permitted by the copyright and related rights legislation that applies to your use. For other uses you must obtain permission from the rights-holder(s) directly, unless additional rights are indicated by a Creative Commons license in the record and/ or on the work itself. This Dissertation has been accepted for inclusion in WVU Graduate Theses, Dissertations, and Problem Reports collection by an authorized administrator of The Research Repository @ WVU.

For more information, please contact researchrepository@mail.wvu.edu. 


\title{
Laboratory and Real-World Measurement of Diesel Particulate Matter
}

\author{
Yuebin Wu \\ Dissertation submitted to the \\ College of Engineering and Mineral Resources \\ at West Virginia University \\ in partial fulfillment of the requirements \\ for the degree of
}

Doctor of Philosophy

in

Mechanical Engineering

Nigel N. Clark, Ph.D., Chair

Benjamin C. Shade, Ph.D.

Hailin Li, Ph.D.

W. Scott Wayne, Ph.D.

Steven E. Guffey, Ph.D.

Department of Mechanical and Aerospace Engineering

Morgantown, West Virginia

2010

Keywords: Diesel, Particulate Matter, Real-World Emissions, Particle Sizing 


\section{ABSTRACT \\ Laboratory and Real-World Measurement of Diesel Particulate Matter}

\section{Yuebin Wu}

West Virginia University's Center for Alternative Fuels, Engines, and Emissions (CAFEE) has designed and constructed, with support from the U.S. Department of Energy (DOE), the 'next level' transportable dual-primary full-flow dilution tunnel emissions-measurement laboratory. As one of the major contributors, the author participated in the design and the fabrication processes of this laboratory. A systematic Simulink ${ }^{\circledR}$ model was built for the Particulate Matter (PM) sampling system and a parametric study of the PM system was performed. Modeling of gas chemical composition, mass and heat transfer, as well as modeling of the primary and the secondary tunnels, were conducted with this Simulink ${ }^{\circledR}$ model. This model simulated the tunnel flow and wall temperature, the PM filter face temperature, and the system's estimated theoretical PM diffusion losses. A computational fluid dynamic (CFD) model was also set up to help the selection of the location and sizes of the mixing orifice plates, as well as the configurations of the high efficient particulate air (HEPA) filter and the exhaust pipe housing plenum box. This dissertation describes the modeling processes and results from this Simulink ${ }^{\circledR}$ model and this CFD model. Each sub-system of this transportable laboratory and results of the qualification tests on the laboratory as the outcomes of the CAFFE teamwork are also described. Size distributions of ultra-fine particles in the diesel exhaust from a naturally aspirated, 2.4-liter, 40-kW ISUZU C240 diesel engine equipped with a diesel particulate filter (DPF) were studied. Tunnel dilution in the standard primary and secondary-dilution tunnels on the transportable laboratory, instrument dilution with one Portable Particulate Measurement Device (PPMD), and ambient dilution at post-tailpipe centerline of the engine were studied as three dilution methods with different dilution ratios. Particle size distribution data, during steady-state engine operation, were collected using a Cambustion DMS500 Fast Particulate Spectrometer. The CFD models were employed to predict the exhaust mixing in the primary tunnel and at the engine post-tailpipe centerline. The dilution ratios obtained from the CFD models were verified with measured dilution ratios. The CFD models then were used to provide auxiliary information on the exhaust dilution processes.

The tunnel test results indicated varying size distributions across the tunnel cross sections where the flow was still developing. Homogenous particle-size distributions were observed across the sections at locations where the primary flow was fully mixed. However, the profile of particle-size distributions continued to evolve, due to residence time, even for fully mixed primary flow conditions. Variability of size distributions at the end of the secondary dilution tunnel was also observed with varied secondary-dilution ratios. The effects of dilution ratios, dilution speeds, and residence times on the diesel particulate matter (DPM) size distributions and particle mass concentration levels were analyzed and discussed. For example, the increased residence time of particles in the primary tunnel resulted in lower total particle count but higher mass concentration level. The particle-size distributions of the post-tailpipe and the PPMD test results were also analyzed and compared to the tunnel test results. The results from the post-tailpipe study show that the particle-number-concentration levels increased along the post-tailpipe centerline, with increasing dilution ratios. It indicates that nucleation was the dominant process when the exhaust plume was diluted along the post-tailpipe centerline. The measurement results from the PPMD dilution indicate that change of particle-size-distribution curves, number and mass concentration levels were not as strongly correlated to the dilution ratios as other two tests indicated. Finally, the analyzed results showed that stack corrected and uncorrected particle-size-distributions obtained from one dilution method could be remapped to particle-size distributions obtained from another dilution method. The particle-size distributions measured inside tunnels could mimic the freshly emitted exhaust immediately post-tailpipe. For example, when the engine was running at $122 \mathrm{Nm}$ and $1800 \mathrm{rpm}$, the stack-corrected particle-size-distribution measurement at $38 \mathrm{~cm}$ of post-tailpipe centerline was found to be identical to the stack-corrected particlesize-distribution measured at the secondary dilution tunnel outlet with the primary-dilution ratio of 12.5 and the secondary-dilution ratio of 8 . 


\section{ACKNOWLEDGEMENTS}

This research was supported by the US Department of Energy, under grants DEFG26-90CH10451 and DE-FC26-08NT01920.

Dr. Nigel Clark, thank you for your friendship and for giving me the opportunity to work on this topic, without your support and advice, none of this could be done. Dr. Benjamin Shade, Dr. Hailin Li, Dr. Scott Wayne, and Dr. Steve Guffey, thank you for serving on my committee, and for providing me with the guidance, support and resources that are necessary for accomplishing my research objectives. Your valuable advice largely improved the quality of this work. Daniel Carder, thank you for your help and it was a pleasure and an honor to work with you. WVU Engine and Emissions Research Center and WVU Transportable Heavy-duty Vehicle Emission Testing Laboratory's staff, thank you for your many years of support and help. Yan and Melody, my wife and daughter, your love and unconditional support helped me to achieve this work. Thanks to all! 


\section{TABLE OF CONTENTS}

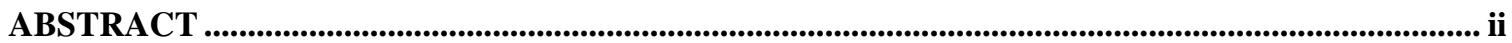

ACKNOWLEDGEMENTS .................................................................................................................... ii

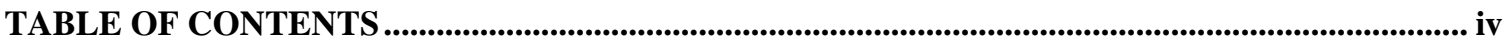

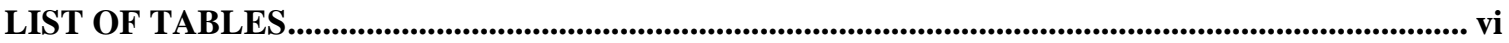

LIST OF FIGURES............................................................................................................................ vii

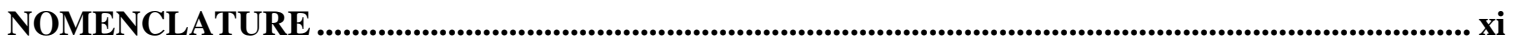

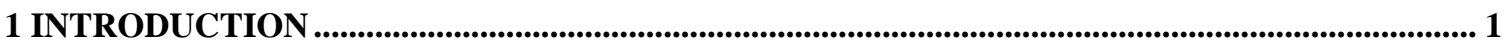

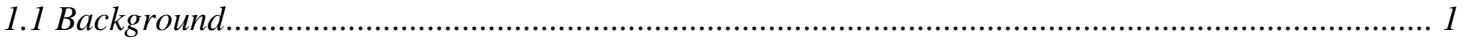

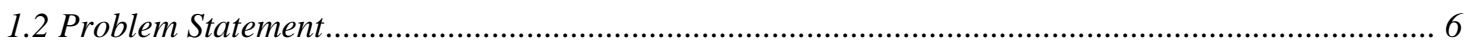

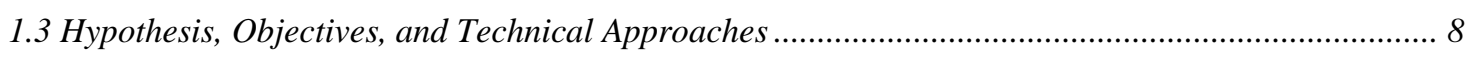

2 REVIEW OF LITERATURE............................................................................................................. 12

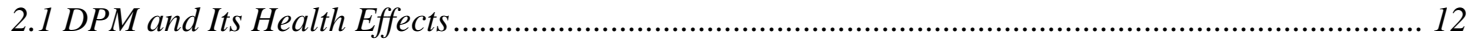

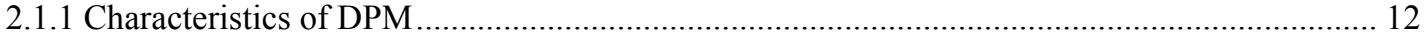

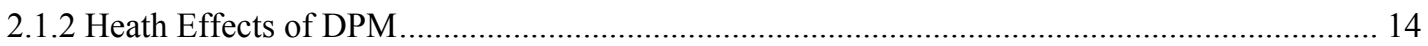

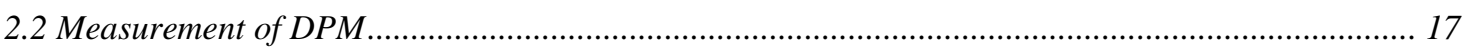

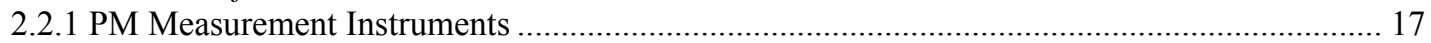

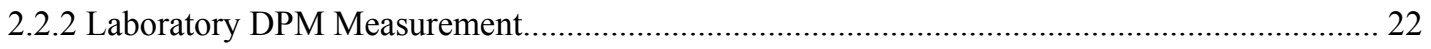

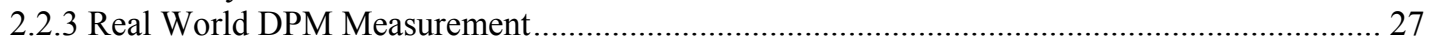

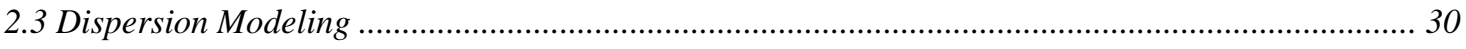

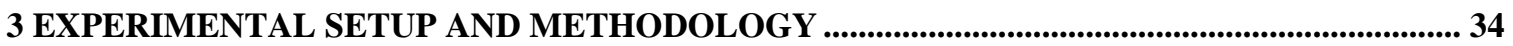

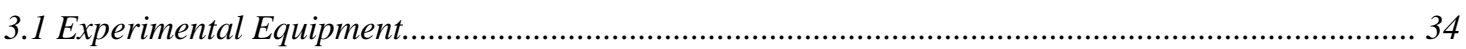

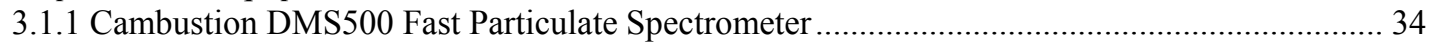

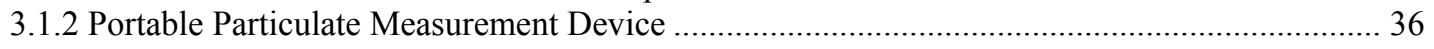

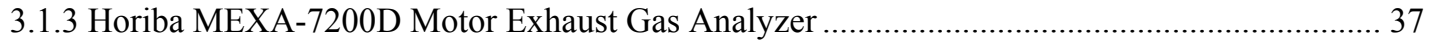

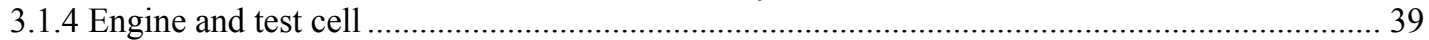

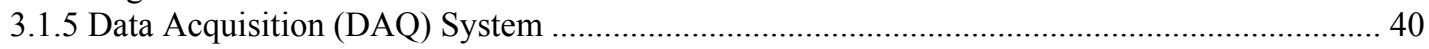

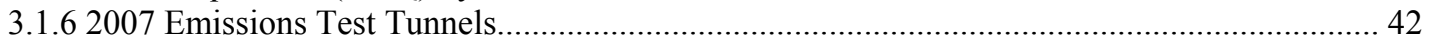

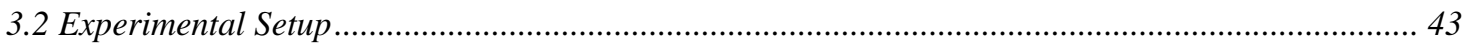

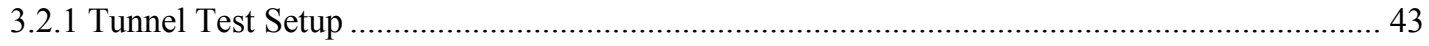

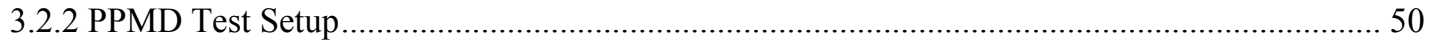

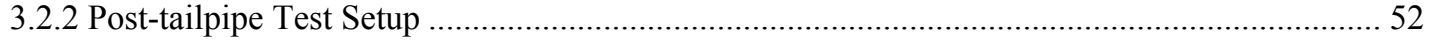

4 TRANSPORTABLE LOW EMISSIONS MEASUREMENT LABORATORY .................................... 55

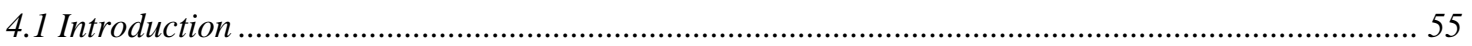

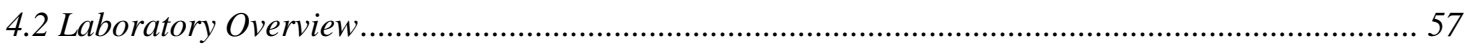

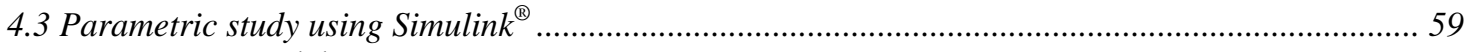

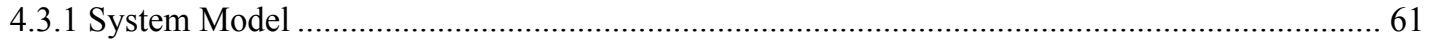

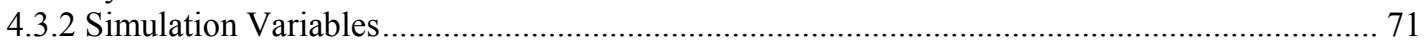




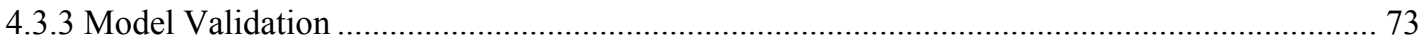

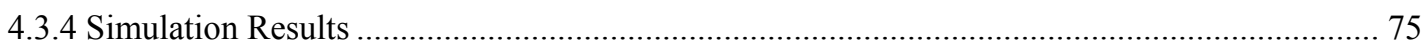

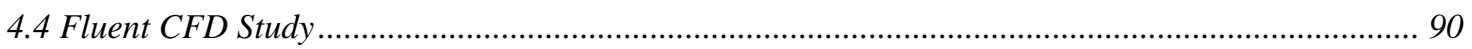

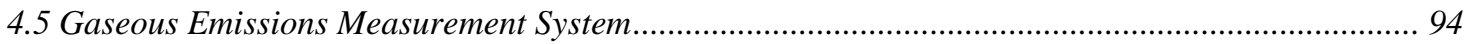

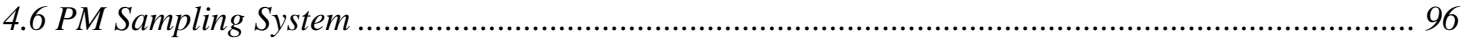

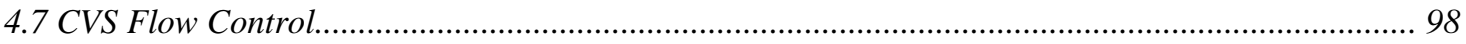

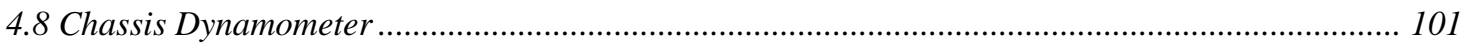

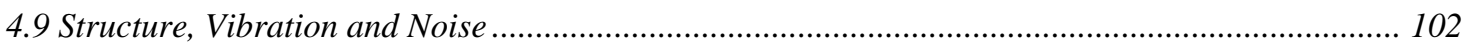

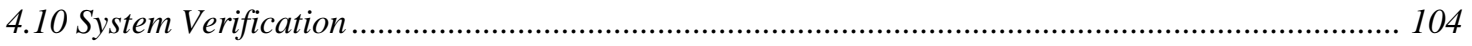

5 ANALYSIS AND EXPERIMENTAL RESULTS................................................................................... 108

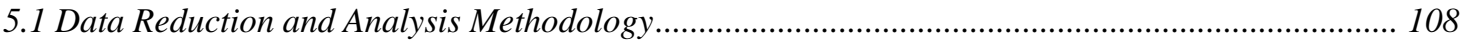

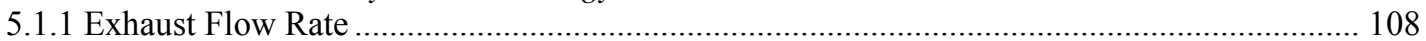

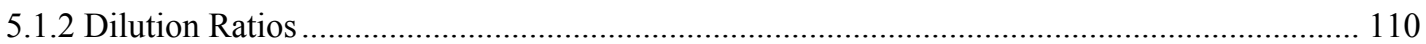

5.1.3 Comparison of Particle Size Distributions ............................................................................. 112

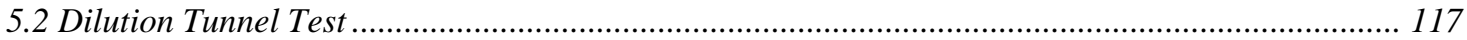

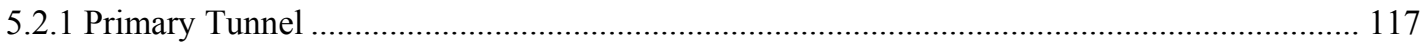

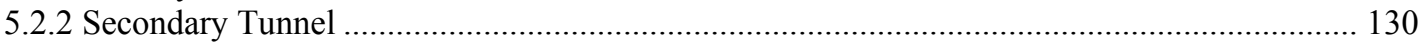

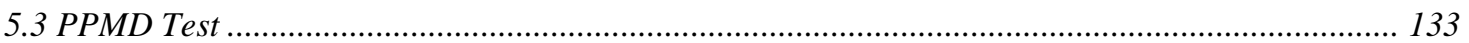

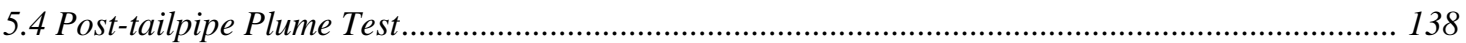

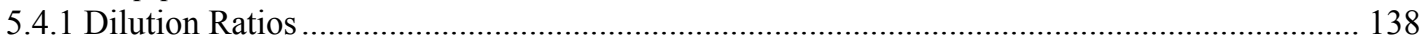

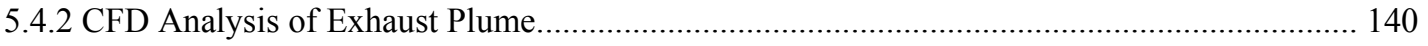

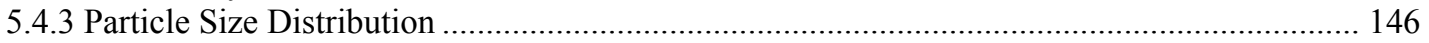

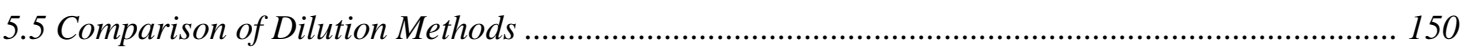

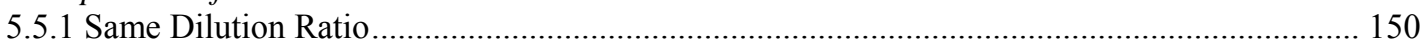

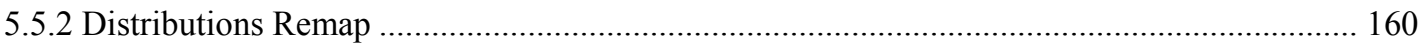

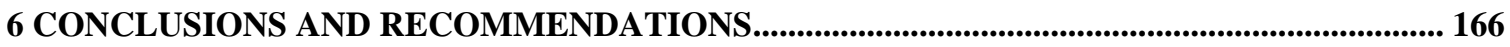

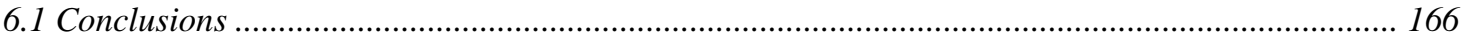

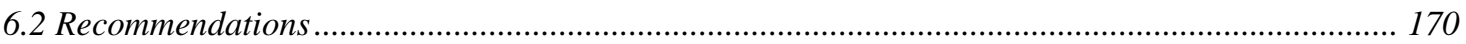

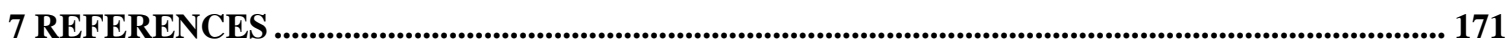

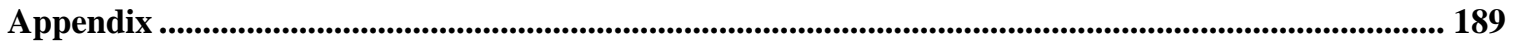

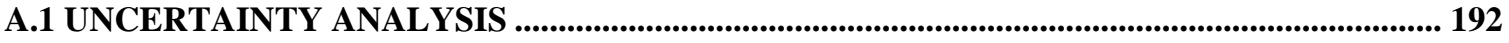

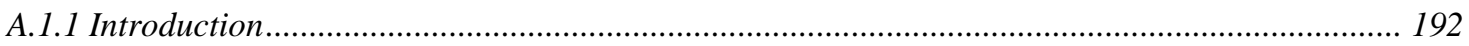

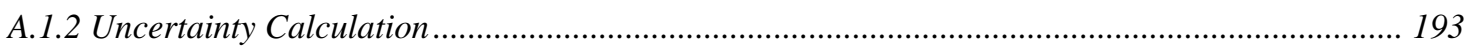

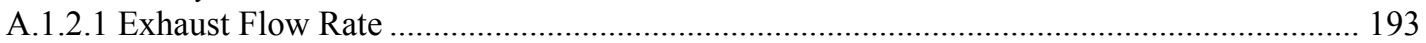

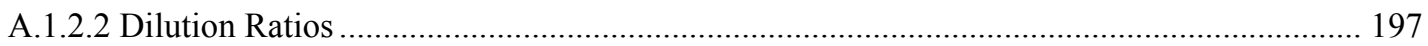

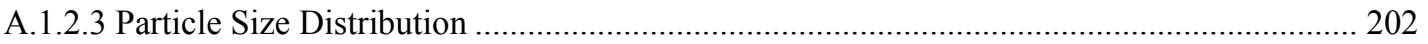




\section{LIST OF TABLES}

Table 1 Particle dynamics and transformation processes and their impacts $[45,117] \ldots . .25$

Table 2 Calibration setting of MEXA-7200D gas analyzer.......................................... 39

Table 3 Historical weather station data on 01/03/2009, South Park, Morgantown, WV . 53

Table 4 Orifice size selection guide....................................................................... 94

Table 5 Fuel consumption rate in SCFM volumetric flow rate ................................... 108

Table 6 Stack uncorrected particle size distributions remap (Kolmogorov Smirnov test)

Table 7 Stack corrected particle size distributions remap (Kolmogorov Smirnov test). 162

Table 8 Stack uncorrected particle size distributions (least square root test) ................ 163

Table 9 Stack corrected particle size distributions remap (least square root test) .......... 163

Table 10 Fuel consumption rate and uncertainty of each steady state........................... 194

Table 11 Sensor specifications for LFE flow rate measurement .................................. 195 


\section{LIST OF FIGURES}

Figure 1 Typical diesel exhaust mass and number weighted PM size distribution [8].... 13

Figure 2 Schematic of particulate emissions sampling system.................................... 23

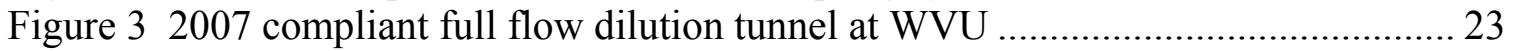

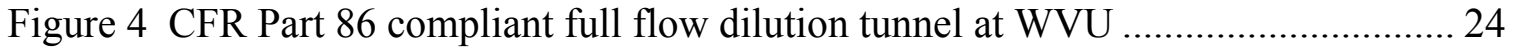

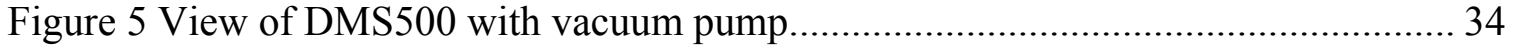

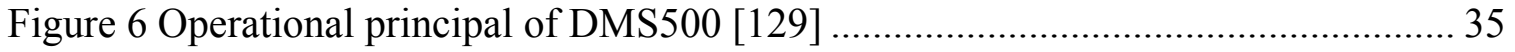

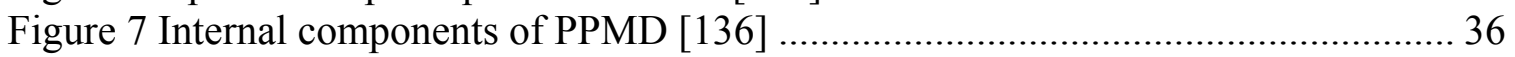

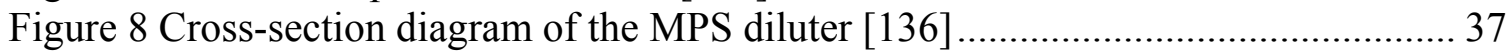

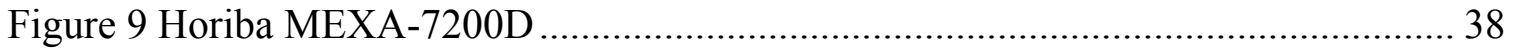

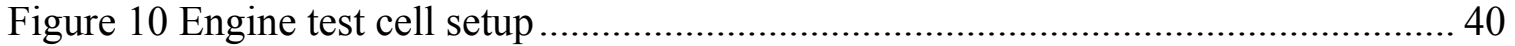

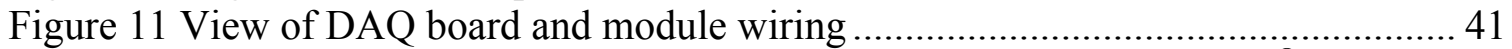

Figure 12 Partial view of the DAQ software program diagram in LabVIEW $^{\circledR} \ldots \ldots \ldots \ldots . . . .41$

Figure 13 Schematic of laboratory emission test tunnels used for testing....................... 42

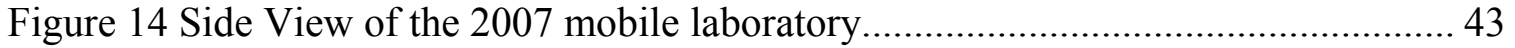

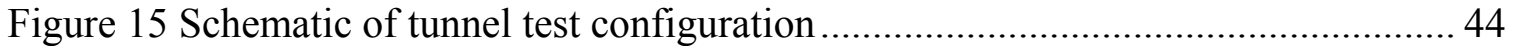

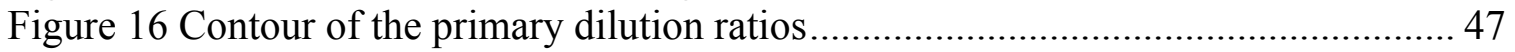

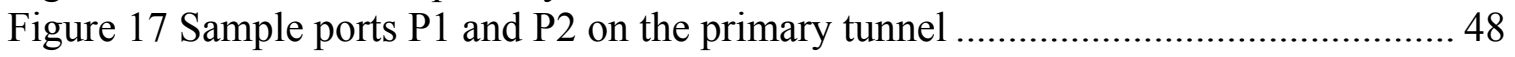

Figure 18 Measured and simulated dilution ratios................................................... 49

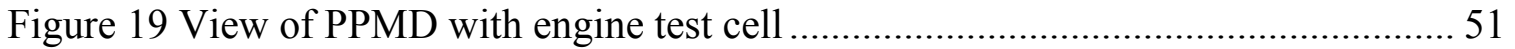

Figure 20 Front and side views of PPMD connections............................................. 51

Figure 21 After exhaust tailpipe test setup with view of the sample probe tip................ 52

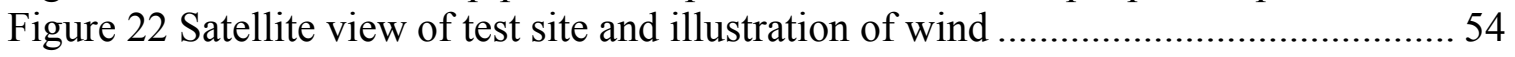

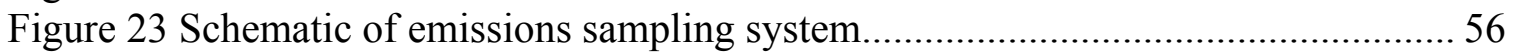

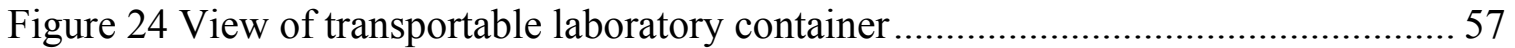

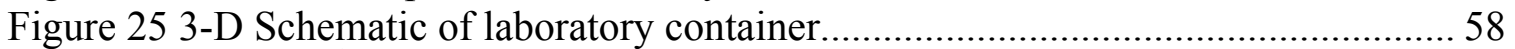

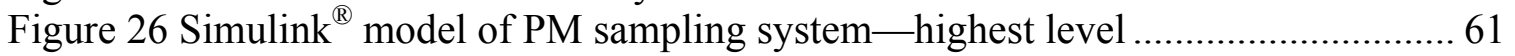

Figure 27 Kinematic viscosity of dry air and exhaust at different air fuel ratios ............ 63

Figure 28 Specific heat of dry air and exhaust at different air fuel ratios ...................... 64

Figure 29 Thermal conductivity of dry air and exhaust at different air fuel ratios.......... 64 Figure 30 Dilution ratios of the primary tunnel flow for a 2004 Cummins ISM370 engine

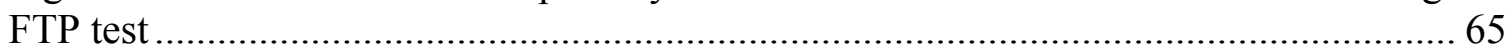

Figure 31 Simulated and measured CFV inlet temperatures ...................................... 74

Figure 32 Simulated and measured filter face temperatures..................................... 75

Figure 33 Maximum and minimum filter face temperatures with different transfer pipe

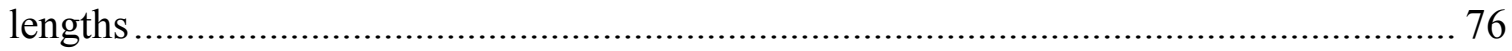

Figure 34 PM penetration percentage distributions with different transfer pipe lengths . 77

Figure 35 Maximum and minimum filter face temperatures with different secondary

tunnel lengths

Figure 36 Real time filter face temperatures for an FTP run with different secondary tunnel lengths

Figure 37 Maximum and minimum filter face temperatures with different secondary

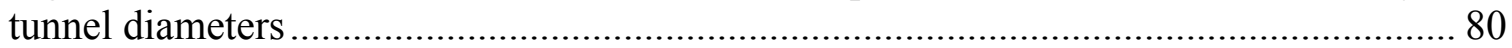

Figure 38 PM penetration percentages with different secondary tunnel diameters......... 81 Figure 39 Heat lost to the outside of the measurement system with different thickness of insulation. 
Figure 40 Cumulative total heat lost to the outside of the measurement system with different thickness of insulation and the cumulative exhaust energy delivered by the engine

Figure 41 Maximum and minimum filter face temperatures with different primary flow

rates 84

Figure 42 Real time filter face temperatures with different primary flow rates 85 Figure 43 Maximum and minimum filter face temperatures when the engine was running between at idle and at full power. 86 Figure 44 Maximum and minimum filter face temperatures with different secondary tunnel flow rates. 87

Figure 45 Maximum and minimum filter face temperatures with different preset secondary wall temperatures.

Figure 46 Real time PM emissions and deposited PM mass on the filter with 2 different CVS system flow rate settings.

Figure 47 Total PM captured with different primary flow rates, secondary flow rates and

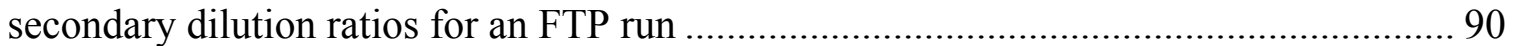

Figure 48 Tunnel model and 3-D/2-D model mesh grids ......................................... 91

Figure 49 Contours of $\mathrm{CO}_{2}$ mass fractions (\%) for different exhaust outlet locations ..... 93

Figure 50 Gaseous emissions measurement system ................................................ 94

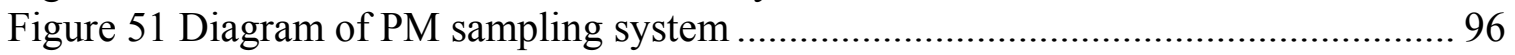

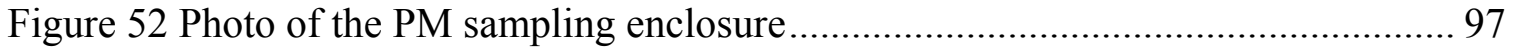

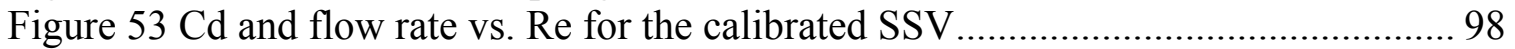

Figure 54 View of the complete flow rate control unit.............................................. 99

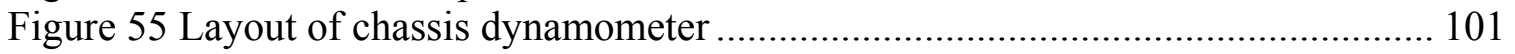

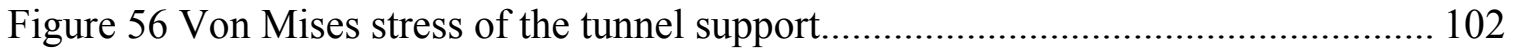

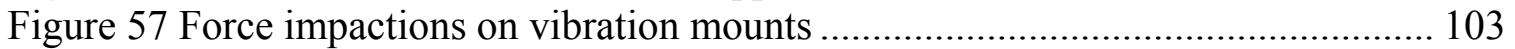

Figure 58 Example of THC analyzer linearization data ............................................ 104

Figure 59 Averaged gaseous emissions of FTP tests............................................... 105

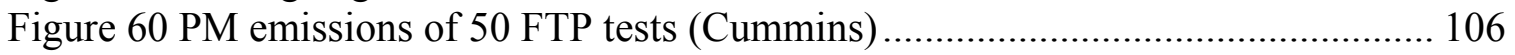

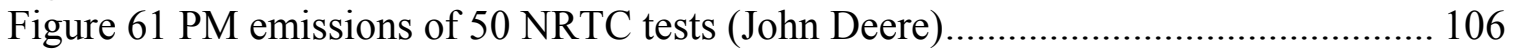

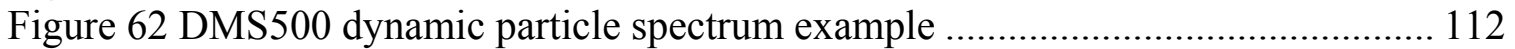

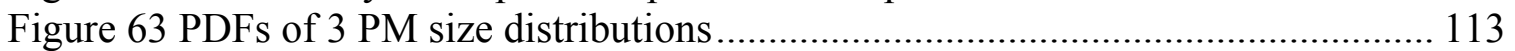

Figure 64 Dilution ratios across tunnel sections .................................................... 118

Figure 65 Particle size distributions of the dilution flow at different sampling distance

from tunnel cross section center at P1 (primary flow rate 0.378 m³ $/ \mathrm{s}, 102 \mathrm{Nm} @ 1800$

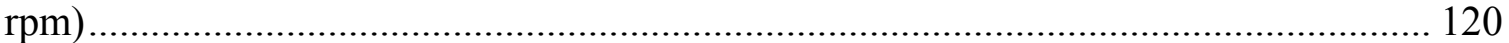

Figure 66 Particle size distributions of the dilution flow at different sampling distance from tunnel cross section center at P1 (primary flow rate 0.944 m³ $/ \mathrm{s}, 102 \mathrm{Nm} @ 1800$ rpm ........................................................................................................... 120

Figure 67 Particle size distributions of the dilution flow at different sampling distance from tunnel cross section center at P1 (primary flow rate 0.378 m³ $/ 122 \mathrm{Nm} @ 1800$ rpm).....

Figure 68 Particle size distributions of the dilution flow at different sampling distance from tunnel cross section center at P1 (primary flow rate 0.944 m³/s, 122 Nm @ 1800 $\mathrm{rpm})$ 
Figure 69 Particle size distributions of the dilution flow at different sampling distance from tunnel cross section center at P2 (primary flow rate 0.378 m³/s, $102 \mathrm{Nm} @ 1800$ rpm)...

Figure 70 Particle size distributions of the dilution flow at different sampling distance from tunnel cross section center at P2 (primary flow rate $0.944 \mathrm{~m}^{3} / \mathrm{s}, 102 \mathrm{Nm} @ 1800$ rpm).

Figure 71 Particle size distributions of the dilution flow at different sampling distance from tunnel cross section center at P2 (primary flow rate $0.378 \mathrm{~m}^{3} / \mathrm{s}, 122 \mathrm{Nm} @ 1800$ rpm)

Figure 72 Particle size distributions of the dilution flow at different sampling distance from tunnel cross section center at P2 (primary flow rate 0.944 m³/s, $122 \mathrm{Nm} @ 1800$ rpm)

Figure 73 Particle size distributions of the dilution flow at P3 and P4 (primary flow rate $\left.0.378 \mathrm{~m}^{3} / \mathrm{s}, 102 \mathrm{Nm} @ 1800 \mathrm{rpm}\right)$

Figure 74 Residence effects on total particle number concentration(102 Nm@1800 rpm)

Figure 75 Residence effects on total particle mass concentration (102 Nm @ 1800 rpm)

Figure 76 Particle size distributions at the end of the secondary tunnel with different secondary dilution ratio (primary flow rate 0.378 m³ $/ \mathrm{s}, 102 \mathrm{Nm} @ 1800 \mathrm{rpm}$ )............. 131 Figure $77 \mathrm{PM}$ mass concentrations with varied secondary dilution ratios (primary flow rate $\left.0.378 \mathrm{~m}^{3} / \mathrm{s}, 102 \mathrm{Nm} @ 1800 \mathrm{rpm}\right)$

Figure 78 Particle size distributions with single MPS dilution stage (102 Nm @ 1800

rpm)

Figure 79 Particle size distributions with single MPS dilution stage (122 Nm @ 1800 rpm)

Figure 80 Particle size distributions with double MPS dilution stage (102 Nm @ 1800 rpm).

Figure 81 Particle size distributions with double MPS dilution stage (122 Nm @ 1800 rpm)

Figure 82 Particle number concentration with PPMD dilution (102 Nm @1800 rpm).. 135 Figure 83 Particle mass concentration with PPMD dilution (102 Nm @1800 rpm)...... 136 Figure 84 Particle number concentration with PPMD dilution (122 Nm @1800 rpm).. 136 Figure 85 Particle mass concentration with PPMD dilution (122 Nm @1800 rpm)...... 136

Figure 86 Dilution ratios of exhaust plume post-tailpipe centerline............................... 139

Figure 87 Dilution ratios of exhaust plume post-tailpipe centerline zoomed view ........ 139 Figure 88 CFD grids of computation domains ……………................................... 141

Figure 89 Meshing of the tailpipe centerline cross section.......................................... 142

Figure 90 Streamline of simulated plume dispersion (running at $102 \mathrm{Nm}$ with $0.44 \mathrm{~m} / \mathrm{s}$

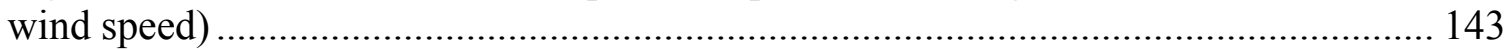

Figure 91 CFD simulation result recording points in an offset area............................. 143

Figure 92 Measured and simulated dilution ratios post-tailpipe centerline (102 Nm @

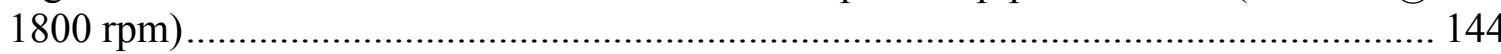

Figure 93 Measured and simulated dilution ratios post-tailpipe centerline (122 Nm @ $1800 \mathrm{rpm})$. 
Figure 94 CFD simulated centerline dilution ratios with different wind speeds (102 Nm (a) $1800 \mathrm{rpm})$

Figure 95 Measured particle size distributions post-tailpipe centerline (102 Nm @ 1800 rpm)

Figure 96 Measured particle size distributions post-tailpipe centerline (122 Nm @ 1800 rpm)....

Figure 97 Particle number concentration post-tailpipe centerline (102 Nm @ 1800 rpm)

Figure 98 Particle number concentration post-tailpipe centerline (122 Nm @ 1800 rpm)

Figure 99 Particle mass concentration post-tailpipe centerline (102 Nm @ 1800 rpm). 149

Figure 100 Particle mass concentration post-tailpipe centerline (122 Nm @ 1800 rpm)149 Figure 101 Particle size distributions of tunnel and post-tailpipe test at dilution ratios of 14 (122 Nm@ @ 1800 rpm)....................................................................................... 151

Figure 102 Particle size distributions of tunnel and post-tailpipe test at dilution ratios of 16 (122 Nm@1800 rpm).................................................................................... 151

Figure 103 Particle size distributions of tunnel and post-tailpipe test at dilution ratios of 26 (122 Nm@1800 rpm)....

Figure 104 Particle size distributions of tunnel and post-tailpipe test at dilution ratios of

31.5 (102 Nm@1800 rpm).

Figure 105 Particle mass concentration levels for tunnel and post-tailpipe test at same dilution ratios

Figure 106 Particle number concentration levels for tunnel and post-tailpipe test at the same dilution ratios

Figure 107 Particle size distributions of tunnel and PPMD test (102 Nm @ $1800 \mathrm{rpm}) 155$

Figure 108 Particle size distributions of tunnel and PPMD test (122 Nm @ 1800 rpm)155

Figure 109 Particle mass concentration levels for tunnel and PPMD test at the same dilution ratios

Figure 110 Particle number concentration levels for tunnel and PPMD test at the same dilution ratios 156

Figure 111 Particle size distributions of post-tailpipe and PPMD test (102 Nm @ 1800 rpm).....

Figure 112 Particle size distributions of post-tailpipe and PPMD test (122 Nm @ 1800

rpm)

Figure 113 Particle mass concentration levels for post-tailpipe and PPMD test at the same dilution ratios

Figure 114 Particle number concentration levels for post-tailpipe and PPMD test at the same dilution ratios

Figure 115 Example of remapped stack uncorrected particle size distributions ............ 164

Figure 116 Example of remapped stack corrected particle size distributions ................ 164

Figure 117 Intake air flow rate and uncertainties over two days of measurement ....... 197

Figure 118 Dilution ratios and measurement uncertainty......................................... 199

Figure 119 Secondary dilution ratios and corresponding uncertainty levels ................. 201

Figure 120 Total secondary dilution ratios and corresponding uncertainty levels ......... 201 


\section{NOMENCLATURE}

ATOFMS

ACFM

$\mathrm{A} / \mathrm{F}$

ANR

AP

CAFEE

CARB

CCRT

$\mathrm{Cd}$

CFD

CFM

CFR

CFV

CMD

$\mathrm{CNC}$

$\mathrm{CNG}$

$\mathrm{CPC}$

$\mathrm{CO}$

$\mathrm{CO}_{2}$

CRC

CRT

CVS

DAQ

DE

DOC

DOE

DMA

DMM

DMPS

DMS500

DISI

DP

DPF

DPM

EAA

EC

EEPS

ELPI

ELR

EMI

EPA

EERL

ESC

FID

FEA
Aerosol Time-of-Flight Mass Spectrometer

Actual Cubic Feet per Minute

Air Fuel

Analyzer Rack

Absolute Pressure

Center for Alternative Fuels, Engines, and Emissions

California Air Resources Board

Catalyzed Continuous Regenerative Trap

Coefficient of Discharge

Computational Fluid Dynamics

Fine Particle Model

Code of Federal Regulations

Critical Flow Venturi

Count Median Diameter

Condensation Nucleus Counter

Compressed Natural Gas

Condensation Particle Counter

Carbon Monoxide

Carbon Dioxide

Coordinating Research Council

Continuously Regenerating Trap

Constant Volume Sampler

Data Acquisition

Diesel Exhaust

Diesel Oxidation Catalyst

Department of Energy

Differential Mobility Analyzer

Dekati Mass Monitor

Differential Mobility Particle Sizer

Differential Mobility Spectrometer

Direct Injection Spark Ignition

Differential Pressure

Diesel Particulate Filter

Diesel Particulate Matter

Electrical Aerosol Analyzer

Elemental Carbon

Engine Exhaust Particle Sizer

Electrical Low Pressure Impactor

European Load Response

Electromagnetic Interference

Environmental Protection Agency

Engine and Emissions Research Laboratory

European Stationary Cycle

Flame Ionization Detector

Finite Element Analysis 


\begin{tabular}{|c|c|}
\hline FTP & Federal Test Procedure \\
\hline $\mathrm{GC} / \mathrm{MS}$ & Gas Chromatography Mass Spectrometry \\
\hline $\mathrm{Gr}$ & Grashof Number \\
\hline GUM & Guide to the Expression of Uncertainty in Measurement \\
\hline $\mathrm{HC}$ & Hydrocarbons \\
\hline HDD & Heavy Duty Diesel \\
\hline HDDE & Heavy Duty Diesel Engine \\
\hline HEI & Health Effects Institute \\
\hline HFID & Heated Flame Ionization Detector \\
\hline HEPA & High Efficiency Particulate Air \\
\hline HVAC & Heating, Ventilating, and Air Conditioning \\
\hline IARC & International Agency for Research on Cancer \\
\hline ID & Internal Diameter \\
\hline IFC & Interface Unit \\
\hline ISO & International Organization for Standardization \\
\hline LDDE & Light Duty Diesel Engine \\
\hline LFE & Laminar Flow Element \\
\hline MCU & Main Control Unit \\
\hline MEL & Mobile Emission Laboratory \\
\hline MOUDI & Micro-Orifice Uniform Deposit Impactor \\
\hline MPS & Micro-Proportional Sampling \\
\hline MUST & Mock Urban Setting Test \\
\hline NAAQS & National Ambient Air Quality Standards \\
\hline NDIR & Non-Dispersive Infrared \\
\hline NI & National Instruments \\
\hline NIOSH & National Institute for Occupational Safety and Health \\
\hline NIST & National Institute of Standards and Technology \\
\hline NRTC & Non-Road Transient Cycle \\
\hline $\mathrm{NO}_{2}$ & Nitrogen Dioxide \\
\hline $\mathrm{NO}_{\mathrm{x}}$ & Nitrogen Oxides \\
\hline $\mathrm{Nu}$ & Nusselt Number \\
\hline NYC & New York City \\
\hline $\mathrm{O}_{3}$ & Ozone \\
\hline $\mathrm{OC}$ & Organic Compounds \\
\hline OCTA & Orange County Transit Authority \\
\hline OEHHA & Office of Environmental Health Hazard Assessment \\
\hline PAH & Polycyclic Aromatic Hydrocarbon \\
\hline PAS & Photoelectric Aerosol Sensor \\
\hline PASS & Photo Acoustic Soot Sensor \\
\hline $\mathrm{PC}$ & Personal Computer \\
\hline PDF & Probability Density Function \\
\hline PDP & Positive Displacement Pump \\
\hline PFSS & Partial Flow Sampling System \\
\hline PID & Proportional-Integral-Derivative \\
\hline PM & Particulate Matter \\
\hline ppm & Parts per Million \\
\hline
\end{tabular}




$\begin{array}{ll}\text { PPMD } & \text { Portable Particulate Measurement Device } \\ \text { PPG } & \text { Project Prairie Grass } \\ \text { Pr } & \text { Prandtl Number } \\ \text { PSL } & \text { Polystyrene Latex } \\ \text { PSU } & \text { Power Supply Unit } \\ \text { PTFE } & \text { Polytetrafluoroethylene } \\ \text { QCM } & \text { Quartz Crystal Microbalance } \\ \text { Ra } & \text { Rayleigh Number } \\ \text { RANS } & \text { Reynolds-averaged Navier-Stokes } \\ \text { Re } & \text { Reynolds Number } \\ \text { RTD } & \text { Resistance Temperature Detector } \\ \text { Sc } & \text { Schmidt Number } \\ \text { SCFM } & \text { Standard Cubit Feet Per Minute } \\ \text { Sh } & \text { Sherwood Number } \\ \text { SI } & \text { Spark Ignition } \\ \text { SHS } & \text { Sample Handling Unit } \\ \text { SMPS } & \text { Scanning Mobility Particle Sizer } \\ \text { SO } & \text { Sulfur Dioxide } \\ \text { SOC } & \text { Soluble Organic Compound } \\ \text { SOF } & \text { Soluble Organic Fraction } \\ \text { SWri } & \text { Southwest Research Institute } \\ \text { SSV } & \text { Subsonic Venturi } \\ \text { SVS } & \text { Solenoid Valve Unit } \\ \text { TC } & \text { Total Carbon } \\ \text { TEOM } & \text { Tapered Element Oscillating Microbalance } \\ \text { THC } & \text { Total Hydrocarbons } \\ \text { ULSD } & \text { Ultra-Low Sulfur Diesel } \\ \text { UV } & \text { Ultra-violet } \\ \text { v-TDMA } & \text { volatility Tandem Differential Mobility Analyzer } \\ \text { WHO } & \text { World Health Organization } \\ \text { WHSC } & \text { World Harmonized Steady-State Cycle } \\ \text { WHTC } & \text { World Harmonized Transient Cycle } \\ \text { WVU } & \text { West Virginia University } \\ & \end{array}$




\section{INTRODUCTION}

\subsection{Background}

Particle pollution, also known as particulate matter (PM), is a generic term for material suspended in the air in the form of small particles and liquid droplets. The size of a particle is described as an in aerodynamic diameter. "The aerodynamic diameter is the diameter of a sphere of unit density, which assumes in a mechanical force field at low Reynolds numbers $(\mathrm{Re})$ the same steady state velocity relative to the gas phase of the aerosol as the actual particle [1]." When these particles are inhaled into a human being's respiratory systems, they can deposit deep inside the lung region, and some of them (several nano meters $(\mathrm{nm})$ in aerodynamic diameter) can even go into the blood system by penetrating across cellular membranes within the lungs [2]. Numerous scientific studies have shown evidence that exposure to PM in ambient air is associated with various adverse health effects ranging from aggravated asthma to increased premature mortality [3-25]. PM pollution is also known to be the culprit for several environmental concerns, such as reduced visibilities in parts of U.S. cities and national parks.

Quantitative information on air particulate pollution toxication has been described as early as being traced back to 1930. In the Meuse Valley, Belgium, a thick mist lasted for three days straight in the surrounding area in early December 1930. It caused several thousand acute pulmonary attacks and 63 deaths. Particles of soot emitted from factories were blamed for this disaster [10]. Burning of low-quality, high-sulfur coal for heating in London caused an up surge of air pollution in early December 1952. In the end, the Great Smog killed 4,000 people in the early weeks and another 8,000 in the following weeks and months $[11,12]$. Those and other catastrophes in modern history have shown the world the astonishing danger of air pollution and motivated the modern environmental protection movement.

To protect public health and welfare, the legislation of air quality standards has been carried out world-wide, and has been piloted by the USA since the US Congress passed the Clean Air Act in 1963. Currently, the United States Environmental Protection 
Agency (US EPA) sets National Ambient Air Quality Standards (NAAQS) for six pollutants. They are PM, ozone $\left(\mathrm{O}_{3}\right)$, carbon monoxide $(\mathrm{CO})$, sulfur dioxide $\left(\mathrm{SO}_{2}\right)$, nitrogen dioxide $\left(\mathrm{NO}_{2}\right)$ and lead. The US EPA first issued standards for PM in 1971 and revised the standards in 1987, 1997 and 2006 based on reviewing scientific studies of PM's health and environmental impacts. The newest 2006 ambient air quality standard regulates two categories of PM pollution: fine particles (PM2.5) (particles are 2.5 micrometers $(\mu \mathrm{m})$ in aerodynamic diameter or smaller) and inhalable coarse particles (PM10) (particles are smaller than $10 \mu \mathrm{m}$ and greater than $2.5 \mu \mathrm{m}$ in aerodynamic diameter). The 2006 standards reduced the 24-hour fine particle standard's allowed levels from 1997's 65 micrograms per cubic meter $\left(\mu \mathrm{g} / \mathrm{m}^{3}\right)$ to $35 \mu \mathrm{g} / \mathrm{m}^{3}$, and the annual fine particle standard at $15 \mu \mathrm{g} / \mathrm{m}^{3}$. The US EPA kept the existing 24-hour PM10 standard at $150 \mu \mathrm{g} / \mathrm{m}^{3}$, and it revoked the annual PM10 standard for lack of sufficient evidence linking long term PM10 exposure to health effects [26]. Air quality standards in Europe require member states of European Union to maintain PM10 concentration level below an annual level of $40 \mu \mathrm{g} / \mathrm{m}^{3}$ and a daily level of $50 \mu \mathrm{g} / \mathrm{m}^{3}$ [27]. Air quality standards in Japan require suspended PM (particles with a diameter smaller than or equal to $10 \mu \mathrm{m}$ ) daily concentration to be below $100 \mu \mathrm{g} / \mathrm{m}^{3}$ and the hourly concentration to be less than 200 $\mu \mathrm{g} / \mathrm{m}^{3}[28]$.

Particulate matter in the atmosphere is either emitted directly from sources ("primary" particulate) or formed from gas condensations, such as Nitrogen Oxides $\left(\mathrm{NO}_{\mathrm{x}}\right)$ and/or oxidants of sulfur in the atmosphere ("secondary" particulate). One of main sources of PM is from diesel combustion of on-road or off-road diesel engines. Diesel Particulate Matter (DPM) is the particulate component of exhaust from diesel engines. It is defined by a prescribed collection method. DPM size distributions are generally distinguished by three modes, which are "nuclei" mode (5 to $50 \mathrm{~nm}$ ), "accumulation" mode or "soot" mode (50 to $500 \mathrm{~nm}$, some literatures range 50 to $300 \mathrm{~nm}$ or 50 to $1 \mu \mathrm{m}$ ), and "coarse" mode $(>1 \mu \mathrm{m})$. For modern diesel systems, the smallest mode (nuclei mode) may account for more than $90 \%$ of the total Diesel particle number while account for only $1 \%$ to $20 \%$ of the total DPM mass. The accumulation mode particles contain the majority of DPM mass, while the coarse mode particles typically account for $5 \%$ to $20 \%$ 
DPM mass with a small number count [8]. PM smaller than $100 \mathrm{~nm}$ in diameter is also defined as "ultrafine" PM, and PM smaller than 50nm in diameter is defined as "nano" PM.

Atmospheric particles are a mixture from multiple sources. As one of the major sources of PM in the atmosphere, DPM has been identified as a toxic air contaminant that can cause several health complications. In 1988, the National Institute for Occupational Safety and Health (NIOSH) classified diesel exhaust (DE) as "a potential occupational carcinogen" [13]. In 1998, the California Air Resources Board (CARB) declared DE to be a Toxic Air Contaminant backed by a detailed investigation conducted by the California Office of Environmental Health Hazard Assessment (OEHHA) [14, 15]. In 1999, the Health Effects Institute (HEI) confirmed the association between lung cancer risk and exposure to diesel emissions [16]. In 2002, US EPA concluded that long-term exposure to DE may "pose a lung cancer hazard to humans" and short-term exposure "can cause irritation and inflammatory symptoms of a transient nature" [17].

The adverse health effects of PM exposure have been shown in many studies. As well as lung cancer, the adverse health effects associated with PM level may include premature death [18] and diseases such as cardiovascular disease [19, 20], stroke [21], respiratory disease [22, 25, and 29], birth defects [30], and impairment of the immune and nervous systems [22]. Most of the studies of environmental exposure of PM have not separated DPM from PM of other sources. DPM's contribution to adverse health effects is supported by studies of human volunteers and animals inhaling diesel engine generated particles [23, 24]. Occupational exposure to DPM is also a known hazard to mineworkers, warehouse workers, railroad workers, and truckers who operate diesel powered equipment. Studies have shown significant increases in the risks of lung cancer $[16,25]$ and permanent central nervous system damage among those workers [22]. Three other specific groups of people are also sensitive to PM pollutions. These include people with pre-existing lung and heart diseases, young children, and the elderly [17, 31]. Recently, special health concerns have been raised for DPM pollution in the ultrafine and nano size particle ranges [25, 31]. 
DPM contributes to about $10 \%$ of total PM2.5 in ambient air and this could go up to an upper bound of $40 \%$ during a severe PM2.5 scenario [32]. In order to achieve the NAAQS PM standards, the US EPA has regulated controllable PM sources, including diesel engines. PM emissions from internal combustion engines have been traditionally regulated based on the total particulate mass. The total exhaust flow is diluted with ambient air in a tunnel which is a part of a device called a "constant volume sampler" (CVS). A secondary probe collects PM by drawing the diluted exhaust through a high efficiency particulate filter. The PM mass deposited on the filter face is determined by comparing the weight of the contaminated filter with that of clean filter. In the mean time, the gaseous emissions, including hydrocarbons $(\mathrm{HC}), \mathrm{CO}$, carbon dioxide $\left(\mathrm{CO}_{2}\right)$ and $\mathrm{NO}_{\mathrm{x}}$, are measured by drawing samples from the diluted exhaust via sample lines to specific analyzers. The requirement of diluting the exhaust first instead of measuring the raw exhaust directly is not out of concerns for technical issues but rather "by the need to express the result in a specific manner" [33].

It would be convenient to use partial dilution tunnels since a partial dilution tunnel is portable compared to the full flow CVS system. Investigation of using partial flow tunnels as an alternative to a full flow CVS has been carried out for several years. In Europe and Japan, the PM measurement using a partial dilution tunnel has also been approved. However, the partial dilution system is more complex and more difficult to calibrate. The US EPA did not approve the use of partial dilution tunnels for heavy duty diesel engine (HDDE) PM emission tests in the 2007 standard. The US EPA determined there was insufficient evidence to support that results form partial dilution tunnels correlated well enough to results form full scale tunnels in measuring diesel emissions. However, use of partial flow dilution tunnels for HDDE emission tests is allowed upon the approval of the US EPA [34].

US EPA regulations require PM emissions measured on the Federal Test Procedure (FTP) cycle from heavy-duty, on-highway diesel engines of model year 2007 or later to be less than 0.01 gram per brake horse power hour (g/bhp-hr). The on-road HDDEs are tested in a computer-controlled test cell. The FTP cycle mimics a vehicle 
undergoing rural driving situations, including steady states, accelerations, decelerations, and overrun conditions. During the test, the entire engine exhaust is injected into a primary dilution tunnel and well mixed with clean dilution air. At the end of the primary tunnel, a fraction of the fully diluted exhaust is drawn into a secondary dilution tunnel and diluted a second time. A PM filter is located at the outlet of a PM cyclone connected to the outlet of the secondary tunnel. The PM emissions mass in $\mathrm{g} / \mathrm{bhp}-\mathrm{hr}$ is calculated based on the PM mass collected on the filter and the engine work over the test cycle. Since 1988, DPM emissions standards for on-road HDDEs have been reduced by $98 \%$ $[35,38]$. The PM emission test procedures for HDDEs are also regulated and available in 40 Code of Federal Regulations (CFR) Part 1065 and 40 CFR Part 86 subpart N.

Most industrialized countries have DPM emission standards equivalently stringent to the US standards. The European emission standards are usually designiated "Euro I" to "Euro VI". Currently, the Euro IV standard regulates the PM emission from HDDEs tested over the European Stationary Cycle (ESC) and European Load Response (ELR) cycles to be less than 0.02 gram per kilowatt hour $(\mathrm{g} / \mathrm{kWh})$. The Euro VI standard (effective 2013.04) will reduce the limit to $0.01 \mathrm{~g} / \mathrm{kWh}$. Environmental legislation in Japan requires PM emissions from on-road HDDEs tested over a JE05 transient cycle (simulates Tokyo driving conditions) to be less than $0.027 \mathrm{~g} / \mathrm{kWh}$ since 2005. For HDDEs of model year 2010 or later in Japan, the standard maximum value is $0.01 \mathrm{~g} / \mathrm{kWh}$ [39]. Many developing countries, such as China and India, employ Euro emissions standards but have five to ten years of effective date lag compared to European Union.

A solid particle number (SPN) emission limit of $6 \times 10^{11}$ particles $/ \mathrm{km}$ becomes effective at the EURO V/VI stages for light duty diesel vehicles. The European Euro VI Certification procedures also require that on-highway heavy duty diesel engines satisfy emissions standards/limits, including particle number count based upon the UN Particle Measurement Program (PMP) protocol. The brake specific particle number limit under trap out conditions for a World Harmonized Transient Cycle (WHTC) cycle has been finalized at $6 \times 10^{11}$ particles/ $\mathrm{kWh}$. The particle number limit was set to be $8 \times 10^{11}$ particles/ kWh for the World Harmonized Steady-State Cycle (WHSC) cycle test. These 
particle number limits have to take into account particles during regeneration similar to how regeneration emissions are taken into account for other emissions species [39].

In 2010, CARB released its proposal for the next tier of emission requirements for light-duty vehicles, known as LEV III. The LEV III proposal does not include a mandatory particle number standard. However, an optional PM number standard of $6 \times 10^{12}$ particles/mile is being introduced for 2014 year vehicles, which could be chosen by manufacturers instead of the PM mass standard. The FTP cycle would be used to measure the SPN using an approach similar to the European PMP methods [39].

To achieve low DPM emission at the 2007 level of the US standard or other counties' comparably stringent standards, the HDDEs are required to be equipped with the DPM aftertreatment equipment. For current production engine technologies, these new DPM standards are unachievable through engine tuning and design only without the employment of the aftertreatment systems. However, the sulfur content of diesel fuel can degrade the performance of advanced DPM pollution control. As regulated by EPA, the sulfur content of on-highway diesel fuel must be less than 15 parts per million (ppm), referred to as ultra-low sulfur diesel (ULSD). ULSD fuel for highway use had been made available in the US beginning in June 2006, which meant the HDDE manufacturers would achieve the 2007 standard with modern DPM pollution control technology. With the implementation of HDDE and diesel fuel regulations, the US EPA investigated the long-term benefits and estimated that 8,300 premature deaths can be avoided and $\$ 70.4$ billion can be saved in 2030 [40].

\subsection{Problem Statement}

Current US federal regulations on PM emissions from diesel engines are measured by an aggregate gravimetric method [34]. However, PM emitted from diesel engines is distributed in different size particles. Toxicology studies have shown that the degree and the type of toxicity of PM particles vary with sizes and chemical compositions [25]. On-road HDDEs which meet the latest PM requirements usually increase the PM emissions in particle number over the nano scale range while decreasing mass emissions 
comparing to older engines which are not equipped with particulate traps. The PM levels emitted from spark ignition (SI) gasoline engine are usually lower than those of diesel engines. However, when the gasoline SI vehicles undergo hard accelerations, the PM size distributions are similar to HDD vehicles equipped with post traps. "Emissions of extremely small particles (diameter $<10 \mathrm{~nm}$ ) at high road speed may be very high $\left(10^{14}\right.$ $10^{16}$ count/kg fuel) even for nominal low emitters" [41]. Recently, special health concerns were raised for PM emissions in the ultrafine and nano scale size range. Concerns about engines with lower PM mass emissions are that they may produce more harmful PM emissions to public health at reduced aggregate mass levels, which defects the purpose of particulate regulations.

The EPA leaves some room for interpretation in the 2007 regulations in CFR subpart $\mathrm{N}$ of part 86, title 40 for 2010 and newer model year HDDEs PM emissions measurement regulation in CFR Part 1065. For example, the EPA does not give specifications for the secondary tunnel's volume and length. It does not state any restrictions on the primary and secondary dilution ratios (at least 2 for primary and 5 to 7 for secondary tunnel in total in CFR Part 1065). There is only an upper bound of 100 $\mathrm{cm} / \mathrm{sec}$ for the filter face velocity. It does not limit the filter media to one specific material though pure polytetrafluoroethylene (PTFE) is recommended in CFR Part 1065. It does not provide an upper limit for the primary and secondary dilution air temperature $\left(25^{\circ} \mathrm{C} \pm 5^{\circ} \mathrm{C}\right.$ in CFR Part 1065). The effects of these factors on PM measurement have been recognized but are still not fully understood.

Various studies have been conducted to investigate the effects of different factors on DPM size distributions, such as dilution process specifics, aftertreatment devices, fuelwater emulsion, and fuel sulfur content levels [42-44]. The use of a partial dilution device for DPM measurement is allowed upon approval from the US EPA. However, the dilution processes of EPA certified partial dilution devices may have different effects on the particle size distributions compared to the dilution processes inside a full scale dilution tunnel. The use of partial or full scale dilution shares the same purpose: to mimic the dilution process of freshly emitted raw exhaust, which is however not "mature" 
atmospheric PM. However, there is little information available regarding DPM size distribution maturation inside full scale primary dilution tunnels. Likewise, minimal published literature has focused on particle size distributions at the DPM sample filter location, after various treatment strategies within secondary dilution tunnels, with partial dilution devices, and at ambient locations after the exhaust tailpipe.

\subsection{Hypothesis, Objectives, and Technical Approaches}

The main objective of this research is to advance the understanding of diesl PM formation and measurement by investigating how the DPM evolves in a full flow CVS tunnel and a partial dilution flow device and how the collected DPM represents the realworld emissions from a diesel engine. The central hypothesis of this proposed research is that the PM size distributions measured inside the full flow dilution tunnel and at the end of the secondary dilution tunnel can be mapped to the PM size distributions at specific locations after the engine tailpipe in the atmosphere. The measurement of PM size distributions after the engine tailpipe is limited to a stationary engine in this research to minimize the influences of the atmospheric variations. The investigation of variables affecting the PM size distribution focused on the effects of the exhaust dilution ratio. The second objective of this research is to conduct a parametric study to quantify the design and operating envelope of a PM measurement system that will allow for compliance with 2007 standards, and to perform a comparison of study predictions with actual tunnel performance. Technical approaches performed to reach the objectives of this study are summarized below:

Phase I: A 2007 compliant dilution tunnel system for the HDDE emissions measurement (the function of this tunnel system is not restricted to 2007 HDDE testing) was designed and built as the platform of the tunnel DPM size distributions measurement in the first phase of the research. In order to assist the design of the tunnel system and analysis of measurement results, models that reasonably represent real dilution behavior in a full scale dilution tunnel were developed. These included a one-dimensional system model for bulk properties and a Computational Fluid Dynamics (CFD) model for detailed study of flow properties. The system model was developed using Simulink ${ }^{\circledR}$ and it is able 
to calculate the diluted exhaust bulk flow properties along the system. Those properties included the flow temperature, dilution ratio, filter face temperature, and particle penetration losses for different dimensional configurations and engine operating conditions. The CFD model was developed in the environment of commercially available software (Fluent) and this model was used to examine the development of the diluted flow. The parametric model and the CFD model were verified against the experimental data measured from the 2007 compliant WVU tunnel during later phases of this research.

Phase-II: The second phase of this research involved the repeatable and reliable PM size distribution and condensation measurement from a diesel engine tested over a 2007 compliant full flow tunnel. In order to perform such a measurement task, preparation work included setting up an engine test system with a diesel engine, an eddy current dynamometer, a digital throttle controller, a digital dynamometer controller, and a data acquisition system for the engine test. Software for engine test control and data logging were developed in National Instruments (NI) LabVIEW ${ }^{\circledR}$. The diesel engine for the test was an ISUZU C240 rated at $52 \mathrm{bhp}$. This engine was operated at different steady state conditions at $1800 \mathrm{rpm}$ for PM size distribution data collection. Control and data logging software was developed for this engine test system. The gaseous emissions concentration levels were monitored during the test. The DMS500 fast particulate spectrometer was used to monitor the PM size distribution in different locations across the tunnel sections and at the end of the secondary tunnel.

The 2007 emission test laboratory has two tunnels available for testing conventional diesel or clean (such as compressed natural gas (CNG)) engines. The test was conducted with a diesel engine so that the diesel emission tunnel (referred as the dirty tunnel) was used. A PM sampling probe was used to sample PM data at 4 different locations after the mixing orifice plate of the dilution tunnel. At each location of sampling, the probe was located at 18 different radial depths across the tunnel crosssections to take the PM size distribution data. A simulation using the CFD model for the dilution tunnel was also performed to predict flow pattern inside the tunnel hence to select appropriate probe locations from the orifice plate. Besides the measurement of PM 
size distributions inside the main tunnel, the PM size distributions at the end of the secondary tunnel were also measured with DMS500. The total engine exhaust flow rates were determined by monitoring the engine intake air flow rate and fuel consumption rate with the blow-by neglected.

Along with the change of the engine running conditions, PM sizing test during this phase was carried out with different primary flow rates and with a Diesel Particulate Filter (DPF). The primary tunnel flow was set to between 800 standard cubic feet per minute (SCFM) and 2000 SCFM. While measuring the PM size distribution of the secondary tunnel, different total dilution ratios were set by varying the secondary dilution ratio.

Phase-III: During the third phase of this research the dilution stages of a Sensors Semtech Portable Particulate Measurement Device (PPMD) was used to dilute the raw exhaust flow and measured the particle size distributions of the diluted exhaust flow. The dilution stages of the PPMD were composed of two Micro-Proportional Sampling (MPS) devices which were capable of providing dilution ratios above 5. The PPMD was configured to provide a single stage and a double stage dilution and the measured particle size distributions were analyzed to investigate the effects MPS dilution.

Phase-IV: The final phase of this research was to measure post-tailpipe PM size distributions of the ISUZU C240 engine. The engine was operated at different steady conditions for the measurement. The sample probe for PM size distribution and gaseous emission levels was located at different locations after the engine's tailpipe centerline. The engine tailpipe was set horizontally and about 1 foot above the ground. Similar to the tunnel measurement test, the purpose of measuring gaseous emissions levels was to use them to determine the dilution ratios of the diluted exhaust. The test date of this phase of testing was carefully chosen so that meteorological conditions had the minimal influence on the exhaust dilution in the ambient condition. The exact locations of the sample probe were determined and adjusted during the test with observed measurement results. The 
modeling part of phase-I was conducted throughout all phases to provide guidance for tunnel design and these research test procedures. 


\section{REVIEW OF LITERATURE}

\subsection{DPM and Its Health Effects}

\subsubsection{Characteristics of DPM}

Diesel emissions are composed of hundreds of organic and inorganic compounds in gas and solid phases. DPM is mainly composed of solid carbonaceous material and volatile and sulfur compounds (at the exhaust manifold upstream of the trap if the engine is trap-equipped and without using ULSD fuel) [45]. Solid aerosol is formed in fuel rich mixture under high temperature during combustion process of the diesel engine. Soluble organic fraction (SOF) or soluble organic compounds (SOCs) is the volatile or semivolatile components of DPM mainly existing in liquid droplet forms. The sources of SOF include engine lubrication oil, unburned fuel, and lower level of partial combustion and pyrolysis products. The percentages of unburned oil and fuel in SOF vary with test cycles and engines [46, 47]. A typical composition of SOF of DPM from non-trap equipped engines is about $25 \%$ unburned fuel and $75 \%$ unburned lubrication oil, with very small fractions of partial combustion and pyrolysis products. The percentage by mass of SOF in DPM can be lower than $10 \%$ or higher than $90 \%$ depending on the engine type and operational condition [48].

Research was conducted by investigators to find the chemical compositions of the organic fraction of DPM. Schuetzle found around $57 \%$ of the extracted organic mass from DPM of a light duty diesel engine (LDDE) is in nonpolar molecules [49]. Pierson and Brachaczek pointed out that the aliphatic $\mathrm{HCs}$ from around $\mathrm{C}_{14}$ to about $\mathrm{C}_{40}$ constitute $90 \%$ of this nonpolar organic fraction [50]. Rogge et al. analyzed the composition of extracted fraction of fine DPM from two 87 model HDDEs using capillary gas chromatography mass spectrometry (GC/MS) [51]. The analysis showed that $90 \%$ mass of the organic compounds (OCs) were unidentified and shown to be mainly branched and cyclic HCs. The rest of the identified OCs mainly consisted of $\sim 60 \%$ n-alkenes, $\sim 20 \%$ n-alkanoic acids, $\sim 3.5 \%$ polycyclic aromatic hydrocarbon $(\mathrm{PAH})$ 
and $\sim 3.3 \%$ oxygen-containing PAH (oxy-PAH). PAHs are known by their mutagenic and carcinogenic character.

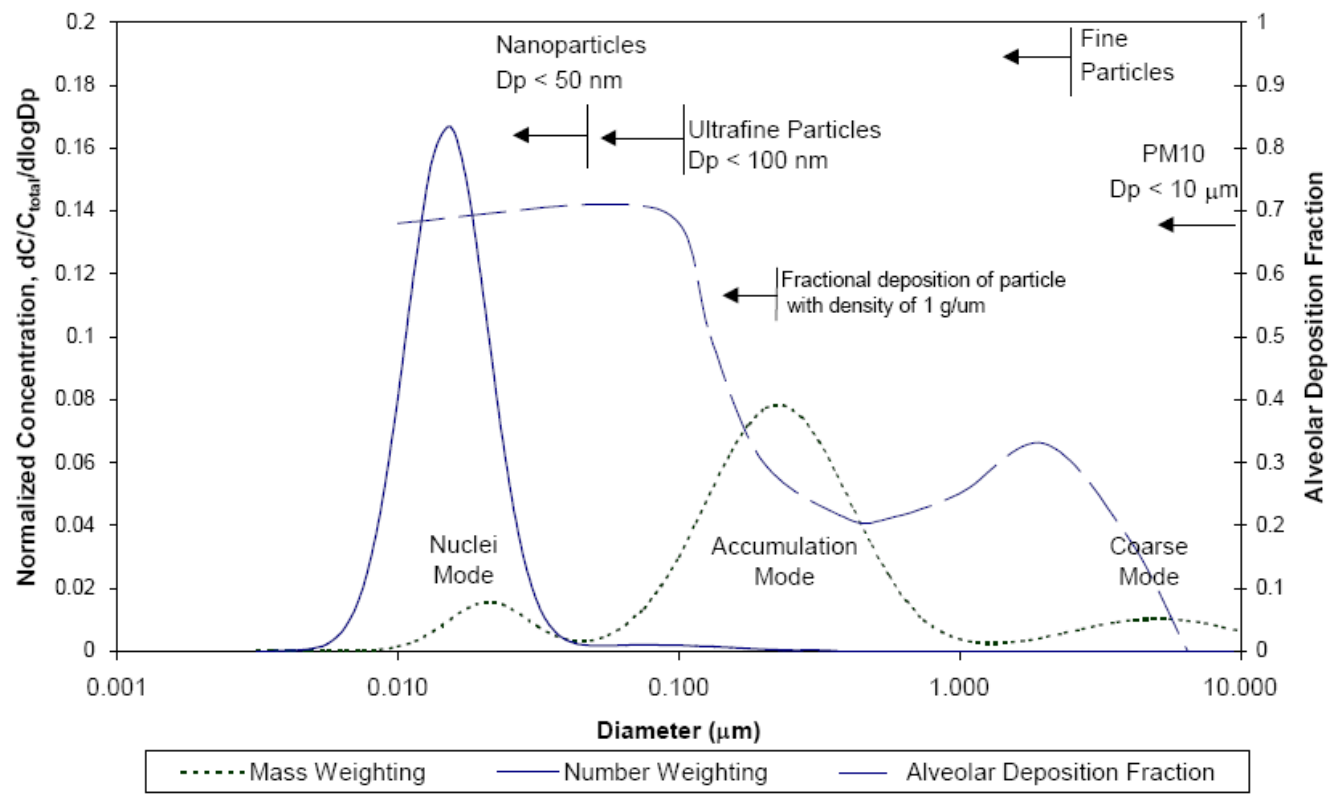

Figure 1 Typical diesel exhaust mass and number weighted PM size distribution [8]

Figure 1 illustrates the typical number and mass weighted size distributions [8, 52] with the alveolar deposition curve [8, 100, 102]. However, the actual DPM size distribution measured from a heavy duty diesel engine may differ substantially from Figure 1. The aerosol dynamics during dilution and sampling are very nonlinear. A minor change of dilution and sampling condition may result in a huge difference in particle size distribution especially for nano size (diameter less than $50 \mathrm{~nm}$ ) particle number. Up to two orders of magnitude of number in nanoparticle count were observed by changing the dilution ratio while keeping identical engine operating conditions [102]. The dilution temperature was also identified as a major factor affecting PM size distribution [11]. Recently, concerns about nano PM from diesel engine have been raised due to the fact that low-emission diesel engines equipped with emission control equipment such as oxidation catalysts or DPFs showed increased number of nanoparticles [53].

Once the primary DPM is emitted from diesel engines into the atmosphere, it undergoes a series of atmospheric transportations and transformations to form secondary DPM. Some active components of DPM will have short lifetimes in the atmosphere 
whereas some more stable components will stay in the atmosphere longer and be transported greater distance. It is essential to better understand the physical and chemical transformation of DPM in the atmosphere for assessing its environmental and health risks [17]. Ultrafine DPM undergoes nucleation, coagulation and condensation to form larger particle in the atmosphere. DPM in the atmosphere can be removed via dry deposition and wet deposition. Wet deposition happens when the Diesel particles act as water vapor condensation nuclei or are washed down by precipitation. Small Diesel particles $(<1 \mu \mathrm{m})$ will stay in the atmosphere longer than the larger particles. Diesel particles usually have residence times of several days [54].

Secondary DPM is formed after the primary DPM has undergone chemical and physical transformation and transportation in the atmosphere. There are no rigid rules to tell when and where the pollutant can be called secondary after emitted from the primary sources. The "aged" exhaust usually refers to exhaust that is more than 1 or 2 days old. The "aging" of DPM varies depending on the environment where the primary DPM undergoes transformations. The toxicity of DPM changes over time during the "aging" process. The overall toxicological consequence of DPM "aging" is not clear because some compounds transform to be more toxic while some become less toxic. Different levels of available reactants in the atmosphere would also alter the toxicological outcome of DPM "aging". For example, a toxicological study showed that DPM exposed to low level of ozone $(0.1 \mathrm{ppm})$ for 48 hours was more toxic than DPM that was not exposed to ozone [55]. The "aged" secondary DPM pollutants are generally more oxidized, hence with more polar fractions and water solubility [56, 57]. However, minimal hygroscopic growth (2.5\%) of diesel particles was observed for freshly emitted DPM [58]. The formation, transportation and concentration of nitro-PAH products in the secondary DPM are one of great concerns because of its more mutagenic character than other PAH products [11].

\subsubsection{Heath Effects of DPM}

As one of the major sources of PM in the atmosphere, DPM has been identified as a toxic air contaminant which can cause various health complications. In 1988, the 
National Institute for Occupational Safety and Health (NIOSH) classified DE as "a potential occupational carcinogen" [13]. In 1989, the International Agency for Research on Cancer (IARC) identified that diesel engine exhaust is probably carcinogenic to humans. HEI and the World Health Organization (WHO) found evidence showing connections between exposure to DE and lung cancer [59, 60]. In 1998, the California Air Resources Board (CARB) declared DE to be a Toxic Air Contaminant backed by a detailed investigation conducted by the California OEHHA [14, 15]. In 1999, the HEI correlated the association between the lung cancer risk and exposure to diesel emissions [16]. In 2002, US EPA concluded that long-term exposure to DE may "pose a lung cancer hazard to humans" and short-term exposure "can cause irritation and inflammatory symptoms of a transient nature" [17].

Many studies have been conducted all over the world to address the effects of daily variations in PM pollution to mortality at low exposure level. WHO summarized the risk estimated from 18 studies conducted in 18 different locations [61]. In those studies, particles in or close to the size range of PM10 were actually measured. There is $0.74 \%$ increase of joint estimated risk on daily mortality (average daily number of deaths) for every $10 \mu \mathrm{g} / \mathrm{m}^{3}$ increased 24-hour average PM10 concentration level. Even though the number of increased risks linked with slight increase in daily PM10 level may look small, the number of deaths estimated to be associated with such risks can be statistically significant on a yearly basis for a large population. Schwartz et al. investigated the health effects of air pollution in six different cities in USA [62]. The results show that the mortality was strongly associated with PM2.5 and PM10 levels rather than particulate level with size between PM2.5 and PM10.

Studies focused on the associations between hospital admissions and air pollution were also conducted over the last decades. Schwartz et al. found hospital emergency room visits for asthma were significantly associated with the previous day's PM10 concentration level in Seattle, Washington [63]. However, analyzed data in this study showed no evidence that sulfur dioxide and ozone were responsible for asthma visits. Stephen et al. investigated the increase of rate of hospitalization and/or emergency room 
visits for respiratory conditions associated with exposure to PM2.5 in the California Central Valley [64]. The study suggested that every 10\% increase of PM2.5 concentration level was associated with a $7.5 \%$ and a $4.1 \%$ increase in chronic and acute respiratory hospitalizations, as well as a $6.5 \%$ and a $5.2 \%$ increase in chronic and acute respiratory emergency room visits.

The adverse health effects of acute and short-term exposure to DE can be summarized as inducing irritations, inflammatory responses in the airways and the lung typical of asthma, and possible immunological and allergenic effects. Dozens of human studies on short-term DE exposure were carried out over past decades as well as animal studies. Rudell et al. conducted several human studies by exposing volunteers to diluted $\mathrm{DE}$ in an exposure chamber. Symptoms of irritation of the eyes and nose and unpleasant smell were reported [65-67]. Kahn et al. and Wade et al. showed occupational short-term overexposure to DE could be responsible for symptoms developed by workers such as persistent asthma, headache, heartburn as well as vomiting [68, 69]. Studies of short-term effects of DE on laboratory animals showed that overload of Diesel particles can be lethal. However, little evidence exists that short-term exposure to DE at lower level impairs lung function [17].

The chronic and long-term exposure to DPM is likely to pose a lung cancer hazard to humans and it may also cause respiratory symptoms, possible neurological and behavioral effects, as well as liver effects. There have been numerous scientific studies indicating that "environmental exposure to DE may present a lung cancer hazard to humans [17]." The particulate fraction of DE holds the most responsibilities for DE's carcinogenic effect. The noncancer health effects of chronic occupational exposure to DE have been evaluated with several epidemiologic studies. Reger et al. studied the respiratory health status of 823 coal miners exposed to DE with 823 miners not exposed to DE, found higher occurrence rates of cough and phlegm for miners exposed to DE than miners not exposed to DE [70]. Gamble et al. reported diesel bus garage workers had a higher incidence of cough, phlegm and wheezing than workers not exposed to DE [71]. However, there was no study with enough scale to look at consistent effect on pulmonary 
function with chronic exposure to DE to date. Several studies were conducted to compare the health effects of whole DE and filtered DPM. Heinrich et al., Brightwell et al. and Iwai et al. evaluated the toxic effects of whole DE and filter DPM on laboratory animals [72-76]. Those studies indicated that Diesel particles are the primary etiologic agents of noncancer health effects in laboratory animals.

Diesel engine laboratory emission studies showed an increase of nanoparticle emissions from low PM emission engines, usually equipped with Diesel Oxidation Catalyst (DOC) and/or DPF [8]. With the enforcement of new emission regulations, special health concerns have been raised for DPM pollution in the ultrafine and nano size particle ranges. Murphy et al. found the smaller particles are more toxic, with a larger surface area, in a carbon black and diesel exhaust particles bioreactivity study [77]. Wichmann et al. illustrated that fine and ultrafine particles were associated with increased mortality. However, the ultrafine particles showed more delayed effects than the fine particles with about four days lag [78]. Renwick et al. showed that ultrafine particles damage macrophage phagocytosis more severely than fine particles compared on a mass basis [79]. Oberdorster et al. demonstrated ultrafine elemental carbon (EC) particle translocated to the liver after one day's inhalation exposure in animal studies [80].

\subsection{Measurement of DPM}

\subsubsection{PM Measurement Instruments}

Dozens of instruments have been developed for PM measurement over past decades [81]. Some of them focus on the measurement of PM mass, and others focus on

the particle number, surface area or chemical compositions. PM measurement usually involves the stage of exhaust dilution. The dilution stage may happen before instrumentation sampling or happen inside of the instrument. Available dilution methods can be summarized as [82]:

- Full flow CVS

- Secondary dilution tunnel

- Partial dilution tunnel (partial flow CVS)

- Ejector dilution system 
- Rotating disc dilution system

- Instrument-internal dilution system

- Raw flow (no dilution / dilution ratio equals one)

The parameters of PM sample measured by the individual instruments can be particle number, size, surface area, composition and mass. Each particle measurement instrument uses one or a combination of following detection techniques to perform the measurements $[81,82]$ :

- Gravimetric microbalance (filter weighting)

- SOF extraction

- Optical (light scattering / attenuation )

- Laser-induced incandescence

- Photo-acoustic absorption

- Oscillating

- Coulometry

- Microscopic

- Gas absorbing

- Differential pressure

- Analytical chemistry

- Condensation

- Aerodynamic mobility

- Electrical mobility

- Diffusion battery

- Radioactive

- Mass spectrometry

The combined method of full flow CVS and gravimetric microbalance for PM mass measurement is the most researched and well known because it is the regulated procedure by the US E.P.A. Instruments used for DPM measurement are briefly discussed in the following list. The listing of instrumentation is not an exhaustive one. However, most commonly seen instruments in the literature study are included. 
Condensation Particle Counter (CPC) [83]: Another name for CPC is Condensation Nucleus Counter (CNC). The particles flow via a saturated vapor and the vapor becomes supersaturated and condenses on the particles once cooled. When the particle diameter grows it can be easily detected with an optical system. CPC's can be operated in counting mode or opacity mode. The counting mode measures light scattering from each individual particle and requires low concentration level. The opacity mode measures high concentration level. It has greater error than the counting mode and needs frequent and careful calibration.

Differential Mobility Particle Sizer (DMPS) [84]: The electrical mobility of charged particles is measured by detecting their drift in an electrical field. The DMPS measures a particle size distribution by increasing the analyzer voltage stepwise. The DMPS scans the size distribution by using a CNC to count the particles pass through the mobility analyzer. Because of the DMPS's relatively long scan time (about 20 minutes) for a complete run, it may have biased results if PM particle concentration and size change during scan.

Scanning Mobility Particle Sizer (SMPS) [85]: The SMPS is similar to the DMPS, but instead it uses a continuous ramp scanning voltage. SMPS significantly reduces the amount of time to finish a size distribution measurement, proximately from DMPS's 20 minutes to be as short as 30 seconds. A SMPS also applies a CNC to counter particles passing through the mobility analyzer.

Electrical Aerosol Analyzer (EAA) [86]: The EAA measures a numberweighted particle size distribution based on particle electric mobility. Charged particles of a specific electric mobility pass through an electrical mobility analyzer and a downstream electrometer measures the current induced by the passed particles. The EAA used to be the most popular instrument used in US but is hardly seen now since the emerging of DMPS and SMPS.

Tapered Element Oscillating Microbalance (TEOM) [87]: The TEOM determines the mass of particles collected on the filter by measuring the change of resonance frequency of the tapered element, which supports the filter. 
Quartz Crystal Microbalance (QCM): The QCM measures the change of resonance frequency of a quartz crystal plate on which particles are deposited by electrostatic precipitation.

Electrical Low Pressure Impactor (ELPI) [88, 89]: The ELPI is a cascade impactor with lowered pressure to decrease lower cut-off diameter. Particles are first charged in a unipolar corona charger then enter a cascade low pressure impactor. The particles are collected on electrically insulated impactor stages and the induced currents on stages are measured with sensitive electrometers. The measured currents are used to calculate the particle number concentration and size based on the known relationship between the collection stage and aerodynamic size of the particles. The scan time of ELPI is as low as 2 seconds.

Differential Mobility Spectrometer (DMS500): Particles are classified with their electric mobility. The DMS500 developed by Cambustion Limited is used in this study. Details of this instrument can be found in Section 3.1.1.

Photo Acoustic Soot Sensor (PASS) [90]: A near infrared light beam irradiates the diluted PM sample. The resulting resonance acoustic wave is measured to determine the black carbon mass in the sample.

Micro-Orifice Uniform Deposit Impactor (MOUDI) [91]: The MOUDI is a 10 stage cascade impactor with the four lower stages having micro-orifice impaction plates. Gravimetric analysis is performed for articles collected on aluminum stages and the mass weighted size distribution is generated. The lower cutoff aerodynamic diameter of the MOUDI is $56 \mathrm{~nm}$. Nano-MOUDI [92] is a modified version of MOUDI. Another four stages with low-pressure operation are added to reduce the cutoff particle size to $10 \mathrm{~nm}$.

Photoelectric Aerosol Sensor (PAS) [93]: A ultra-violet (UV) light irritates the particles to emit electrons and the particles become positive charged. The emitted electrons are quickly removed by an electric field and the charge on particles is measured by an electrometer when the particles are collected on a filter. The measured photoemission is linearly related to the particle surface area and the quantity of particlebound PAH.

Aerosol Time-of-Flight Mass Spectrometer (ATOFMS) [94, 95]: The ATOFMS is an instrument capable of measuring both particle size and chemical 
composition up to 600 particles per minute. The aerodynamic mobility of particles is first measured by counting the time of particles flight between two lasers. The particles then are evaporated by a strong laser and the chemical compositions are analyzed with a mass spectrometer.

Epiphaniometer [96]: The epiphaniometer is an instrument that measures the surface concentration of particles in the nuclei and accumulation mode. The particle surface concentration is determined by counting the number of attached lead atoms produced by a radioactive source.

Research focused on evaluating performances of different instruments was also carried out over past decades. Maricq showed good measurement agreement exists between SMPS and ELPI [97]. However, Coen et al. indicated that both the SMPS and the ELPI underestimate the actual size of Diesel particles but the ELPI gives a more realistic estimation of the actual particle size.

Andersson et al. reported the performance of regulated filter method, SMPS, CPC, QCM, TEOM and DMS [98]. Agreements between QCM and regulated PM mass were found in this study. Mohr et al. compared twenty-one PM measurement systems to each other [82]. Based on data collected, this study suggested four major criteria (sensitivity, reproducibility, health impaction relevance, and calibration traceability) for selecting an instrument.

Khalek reported the DPM measurement comparison with the regulated filter method, the engine exhaust particle sizer (EEPS, similar to DMS), SMPS, Dekati mass monitor (DMM-230) and QCM [35]. Good correlation was observed between filter method and EEPS, DMM-230 and SMPS. However, the EEPS gave a higher estimation of ultrafine particle concentration than the SMPS. The QCM showed early saturation and inconsistency during the measurements and its results were not reported.

Price et al. compared the measurements of PM from a Direct Injection Spark Ignition (DISI) vehicle with DMS500, Dekati ELPI, TSI CPC and AVL PASS [100]. The 
instruments were found to be correlated against each other. However, the correlations were found to be slightly weaker than measurements made on diesel engines as observed in the literature.

\subsubsection{Laboratory DPM Measurement}

Although the EPA reduced legal PM emission levels, most of the measurement system layouts for HDDE emission certification have remained the same [2]. For example, a primary tunnel is used to dilute the full flow of engine exhaust for the 2007 compliant tunnel at WVU, and the diluted engine exhaust is drawn by a blower through a subsonic venturi. At the primary tunnel's entrance, the entire engine exhaust is injected via an exhaust transfer pipe into the primary tunnel upstream of a mixing orifice plate. The exhaust transfer pipe is "the total length of tubing from the exit of the engine exhaust manifold, turbocharger outlet, or after-treatment device to the primary dilution tunnel" [38]. The streams of diluted air and engine exhaust are merged in the mixing region downstream from the orifice plate. At a downstream distance of about 10 times of the tunnel diameter, sample probes are installed to collect diluted gaseous exhaust samples, which are then monitored by instruments capable of continuous analysis. At the end of the sampling region, diluted exhaust is drawn into a secondary dilution tunnel. Conditioned dilution air is also injected into this secondary tunnel. The double-diluted PM sample flow is drawn though a cyclone separator that removes mechanically generated coarse particles. The flow is then drawn across a PM collection filter, which enables the identification of the mass of DPM collected during a test cycle via gravimetric analysis. After flowing across the filter, the PM sample flow is pumped back into the primary tunnel sometimes. Figure 2 shows the schematic of the 2007 standards compliant PM sampling system at WVU. This system is based on the subsonic venturi constant volume sampler (SSV-CVS) concept. A critical flow venturi (CFV) is used at WVU in the older CFR Part 86 system. Figure 3 and Figure 4 shows the new 2007 compliant emissions sampling tunnel and the old emissions sampling tunnel at Engine and Emissions Research Laboratory (EERL) of WVU. The 2007 compliant emission sampling system was designed for but not limited to the testing of HDDEs. With appropriate equipment, instruments and test cycles, it can be used for emissions tests of 
engines or vehicles such as non-road diesel engines, marine diesel engines, and large non-road spark-ignition engines.

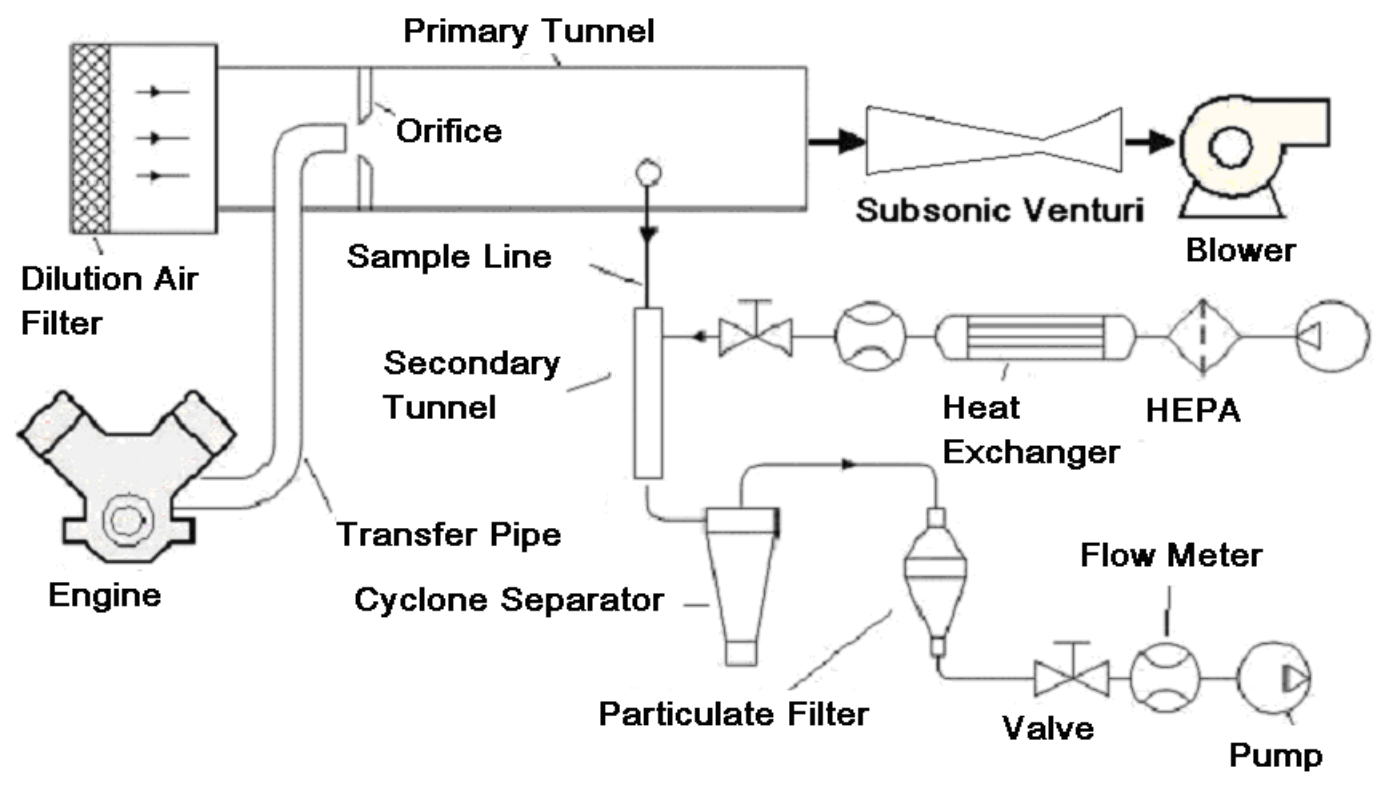

Figure 2 Schematic of particulate emissions sampling system

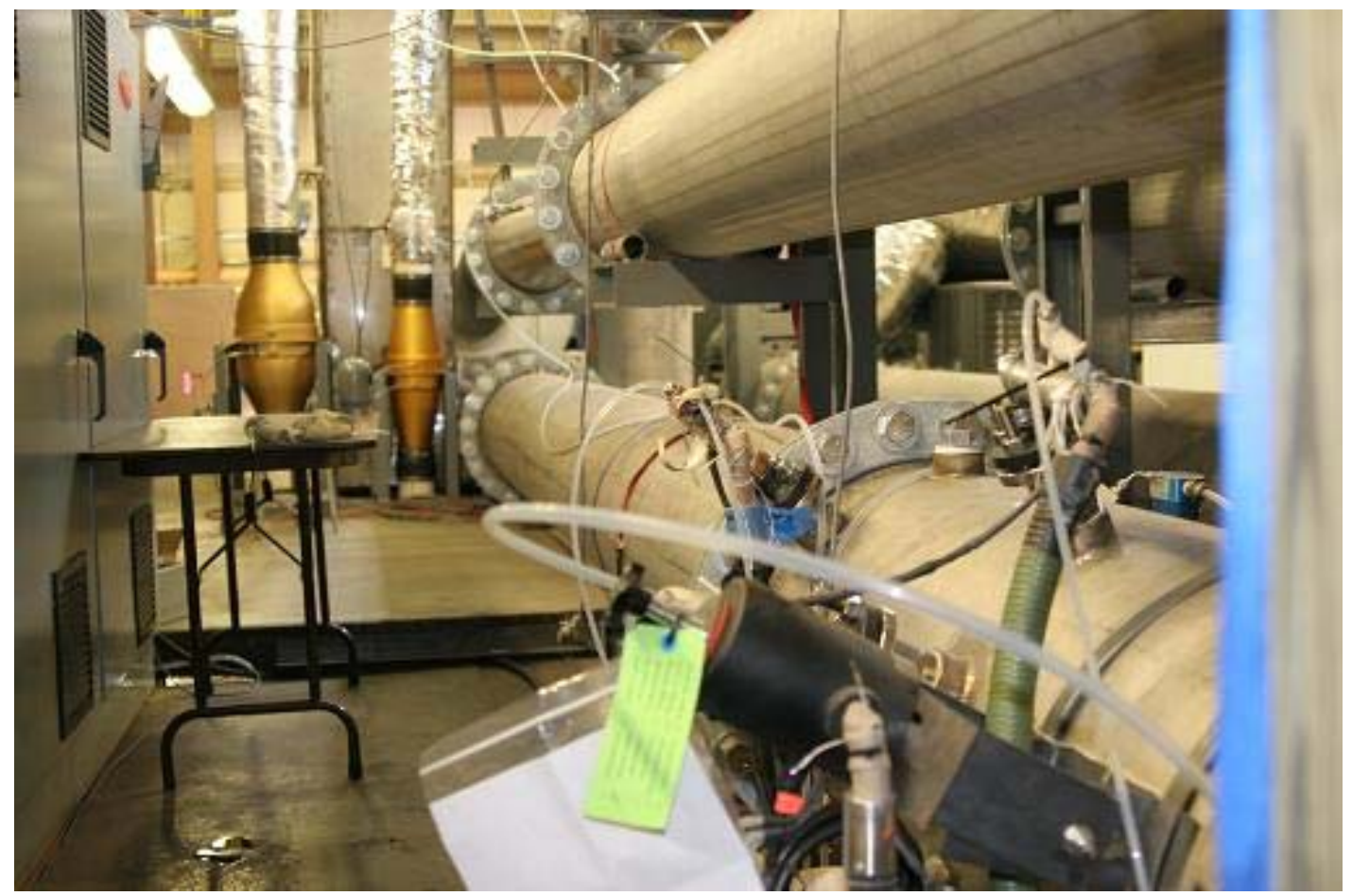

Figure 32007 compliant full flow dilution tunnel at WVU 


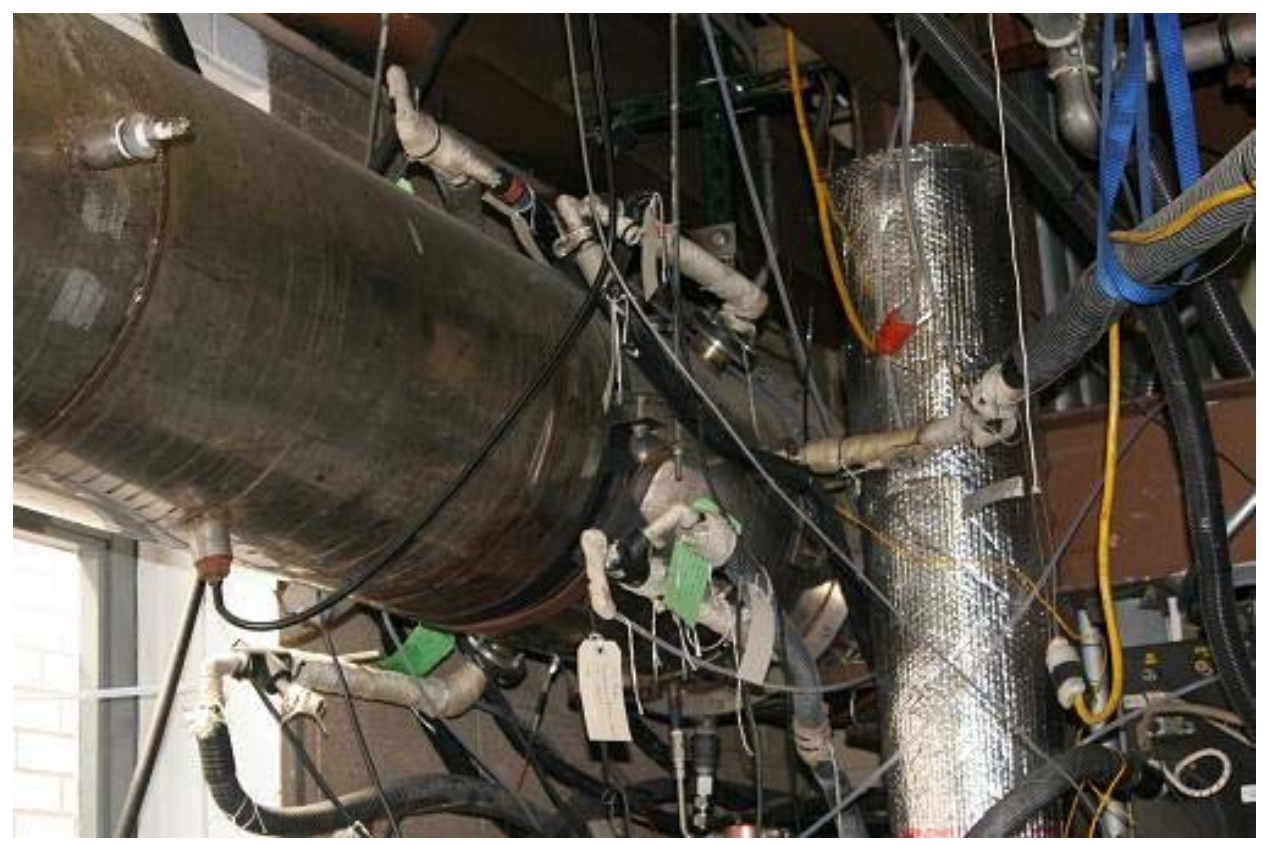

Figure 4 CFR Part 86 compliant full flow dilution tunnel at WVU

The walls of sampling system interact with DPM aerosol during measurement and those interactions could cause the losses of particles. Kittelson et al. summarized the particle-wall interaction mechanisms as of inertial impaction, electrostatic deposition, thermophoretic deposition, diffusion deposition and gravitational deposition [45]. Recommendations to minimize the particle-wall losses in this report include avoiding sharp changes in bending angles of sample lines, avoiding Teflon sample lines, thermally insulating sample lines, using short sample lines and avoiding unnecessarily long horizontal section of sample lines.

Particle dynamic and transformation behaviors could also have impacts on the DPM measurement. Those processes include coagulation, adsorption/desorption, nucleation and condensation/evaporation. The impacts of those processes are summarized and shown in Table 1.

Dilution ratio and resulting saturation ratio, residence time, humidity and temperature have been identified as key parameters affecting DPM emission measurement $[45,48]$. Dolan et al. frequently observed nuclei mode of DPM when the diesel exhaust is rapidly diluted in the atmosphere or inside the dilution tunnel [104]. Nuclei mode of DPM is formed by nucleation and it is triggered with higher saturation 
ratios. Kittelson and Dolan showed the highest saturation ratios reached with dilution ratios of 5 to 50 for two representative condensable hydrocarbons of a diesel exhaust [105].

Table 1 Particle dynamics and transformation processes and their impacts $[45,117]$

\begin{tabular}{|c|c|}
\hline Process & Impact \\
\hline Particle coagulation & 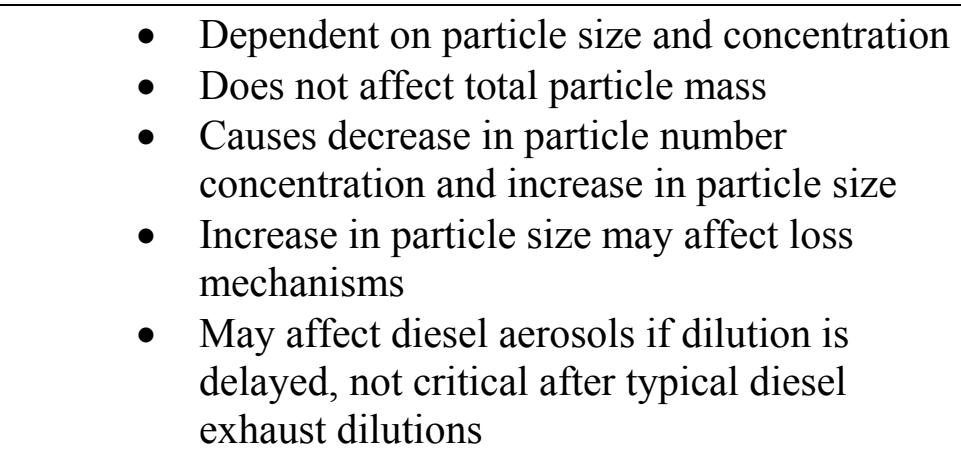 \\
\hline Adsorption/desorption & $\begin{array}{l}\text { - Adsorption/desorption of volatile } \\
\text { components will affect size and mass of } \\
\text { measured particulate matter } \\
\text { - Availability of particulate surface will affect } \\
\text { degree of adsorption/desorption } \\
\text { - Driven by saturation ratio }\end{array}$ \\
\hline Nucleation & $\begin{array}{l}\text { - Homogeneous nucleation may create large } \\
\text { numbers of new particles } \\
\text { - Nucleation rates are highly nonlinear } \\
\text { functions of saturation ratio } \\
\text { - Heterogeneous nucleation leads to the growth } \\
\text { of existing particles } \\
\text { - Increases number and volume concentration } \\
\text { - Increase mass concentration }\end{array}$ \\
\hline Condensation/evaporation & 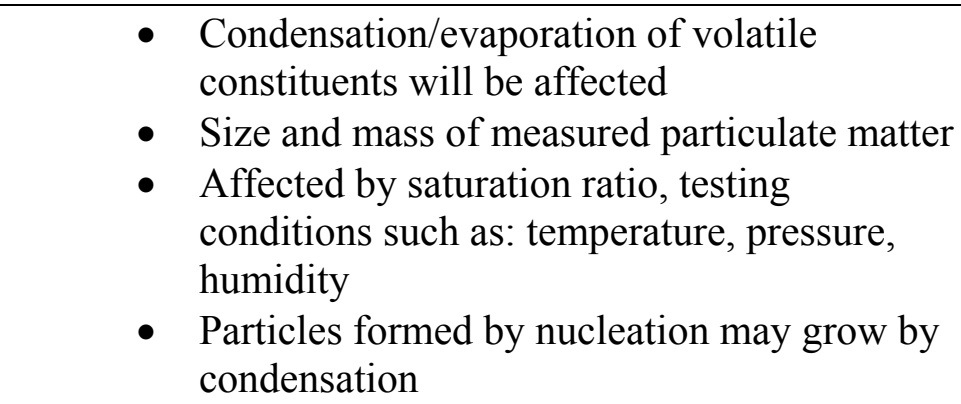 \\
\hline
\end{tabular}


An intermediate residence time also favors particle nucleation. A dilution rate that is too slow would reach the characteristic times of absorption and the nucleation would be suppressed. Kittelson showed a typical characteristic time of adsorption/condensation as of $0.27 \mathrm{~s}$ [105]. A dilution rate that is too fast would not give enough time for nucleation to happen. Khalek et al. reported the nuclei mode number concentration increased by 4 orders of magnitude when the primary residence time increased from 40 ms to $6 \mathrm{~s}[106]$.

The characteristics of engine emissions also play an important role on the DPM emissions. Diesel engines of older technologies have higher concentrations of carbonaceous agglomerates and hence have larger available absorption surface areas. Newer engine technologies (post-1990) largely reduced the DPM emission in carbonaceous part rather than in volatile material fraction. DPM emissions of older engines with higher absorption surface areas reduce saturation ratios by VOF absorption and prevent triggering the particle nucleation due to saturation ratios that are not high enough. The newer engines emit DPM emission with little surface available for absorption and hence nucleation is more likely to happen [45, 48].

Filter media play a role in the DPM capture efficiency. Chase et al. reported organic vapor artifact deposition differences of two different filter media: X40 (Teflon coated fiberglass) and Teflon [107]. The deposited organic artifacts accounted for 10 $20 \%$ deposited PM mass for Teflon filter and 30-50 \% for TX40 filter. An earlier study comparing filter media, TX40 and T60A20, showed a statistically insignificant difference of $0.3 \%$ in PM capture efficiency [107].

The most recent comprehensive DPM measurement study for DPM emission at near or well below 2007 level was the Coordinating Research Council (CRC) sponsored project E-66 conducted by the Southwest Research Institute (swri) [35-37]. The investigated effects of factors on DPM emissions included filter media, filter face velocity, dilution conditions (dilution ratio and residence time), and performance of different instruments. Five different partial flow sampling systems (PFSS) were also 
studied: an AVL-SPC, a Cummins-AEI, a Horiba-MLDT, a Sensor micro proportional sampling (MPS) system and a Sierra-BG3.

The results of the E-66 study suggested using the Teflon membrane filters with narrowed filter face velocity to reduce laboratory to laboratory measurement variations [35]. The primary and secondary dilution ratio and residence time showed significant influence on the real time measurement of PM emissions. A lower dilution ratio and a longer residence time lead to higher PM emissions due to the higher saturation pressure ratio and more time allowed for particle nucleation and growth [36]. All five PFSS systems were able to follow a transient cycle with $5 \mathrm{~Hz}$ data and the measurements yielded similar PM results as the secondary dilution sampling system with a full flow CVS [37].

\subsubsection{Real World DPM Measurement}

Several studies focused on the ambient fine particle and DPM concentrations in urban areas were carried out during last decades. Gray et al. conducted an ambient particle monitoring study across 10 monitoring sites in Los Angeles County, California in 1982 [108]. It was found that carbonaceous aerosols typically constituted of about 40\% total fine particle mass across most monitoring sites. The ratio of total carbon (TC) to EC was used as an indicator of strength of the secondary organic aerosol formation. The primary source emissions were observed to be the overwhelming contributor to the longterm fine particle concentrations and secondary organic aerosols formation was weak during 1982 in this area. The annual average outdoor EC concentrations were 3 to $5 \mu \mathrm{g} / \mathrm{m}^{3}$ in 1982. The majority of EC emissions were estimated to be emitted from diesel engines. The estimated average total DPM concentration level of 1982 in Los Angeles area was $5.7 \mu \mathrm{g} / \mathrm{m}^{3}$ while EC emissions accounted for $59.35 \%$ of the total DPM mass [109].

Maricq et al. compared PM size distributions measured from the standard laboratory dilution tunnel with PM size distributions measured direct at the tailpipes from gasoline and diesel engines [110]. The gasoline vehicles showed similar PM number and size results between tunnel and tailpipe measurements for transient cycles. Distortions 
were observed for Diesel particle size distribution measurements using a dilution tunnel compared to measurements made at the tailpipe with transient cycles. Sampling probes, transfer pipe and dilution tunnel were recommend to be kept clean for DPM tunnel measurement. Materials similar to silicone rubber were identified to be unsuitable for the exhaust transportation lines.

Kinney et al. studied the ambient PM2.5 and DPM concentrations from 4 sampling sites on Harlem sidewalks located at the dense urban core neighborhood of New York City (NYC) [111]. Moderate site to site concentration variations (37 - 47 $\mu \mathrm{g} / \mathrm{m}^{3}$ ) were reported for PM 2.5. However, the EC concentrations varied 4 times from site to site $\left(1.5-6 \mu \mathrm{g} / \mathrm{m}^{3}\right)$. Results of this study indicated the strong association between EC concentrations and local diesel engine emissions sources.

Kittelson et al. measured on-road particle size distributions from the exhaust plume of heavy-duty diesel vehicles powered by a 1988 Cummins L10 engine and a 1999 Cummins LSM engine under the CRC E-43 program [8]. A mobile emission laboratory (MEL) was designed and built to measure diesel PM exhaust in chase experiment under real highway running conditions. The roadway experiments of PM size distribution measurement were well replicated for a given operating and sampling environment. The results showed more nuclei mode particles were produced for the cooler day, which identified the ambient temperature as the main sampling parameter to influence the size distribution.

Vogt et al. measured the exhaust plume particle size distributions by chasing a diesel vehicle cruising on the highway and compared the results with the laboratory measurement of the same vehicle and same speeds [112]. The measurement of the particle size distributions showed good agreements for the soot mood particles. The nucleation mode appeared for the exhaust plume measurement on highway with 360ppm sulfur fuel and a regular oxidation catalyst at 100 and $120 \mathrm{~km} / \mathrm{h}$. However, the nucleation mode disappeared with 10ppm sulfur fuel or without the oxidation catalyst installed. 
Gertler et al. reported an investigation on real-world PM and gaseous emissions from motor vehicles in a highway tunnel funded by HEI [113]. PM emissions in the Tuscarora Mountain Tunnel located on the Pennsylvania Turnpike were studied and compared with measurements over a 25-year period. A dramatic reduction of PM mass emissions was observed over 25 years. The study also showed that diesel engines emitted more PM emissions than did gasoline engines on the mass per mile basis and the ultrafine PM dominated the PM particle number from both sources. The HD and LD PM emissions were separated by applying regression analysis over the range of fleet composition. The observed PM2.5 mass emission factors for HD and LD vehicles were $0.217 \pm 0.029 \mathrm{~g} / \mathrm{mile}$ and $0.022 \pm 0.021 \mathrm{~g} / \mathrm{mile}$, respectively. The chemical species measurement revealed that Organic Carbon (OC) and EC constituted 66\% and 11\% of LD PM2.5 mass, and $49 \%$ and $48 \%$ of the HD PM2.5 mass. PM particle size distributions were also measured during this study. The Count Median Diameter (CMD) of particle size distributions was 17 to $16 \mathrm{~nm}$ with periods of higher HD vehicle fraction ( $65 \%$ to $79 \%$ ). Whereas with periods dominated by LD vehicles ( $85 \%$ to $87 \%)$, the CMD was smaller at $13 \mathrm{~nm}$.

Hasegawa et al. studied the contributions of vehicle traffic activities and meteorological conditions to the nanoparticle number and size distributions from roadside atmosphere in early autumn and winter [114]. Under conditions of low, increased total traffic and/or increased diesel engine vehicles in traffic the number concentration of the 18.4-50 nm particles increased in the morning. Such a rise in PM concentration level was accompanied with the increased level of $\mathrm{NO}_{\mathrm{x}}$. A bi-modal size distribution of ultrafine particles was observed. The first peak appeared at around $30 \mathrm{~nm}$ and the second peak was observed at around $90 \mathrm{~nm}$. The first peak particles were regarded as originating from vehicle emissions and the second peak particles were considered mainly to consist of background particles, since the second peak remained at midnight and on Sunday. A thermal denuder test showed that the first peak consisted of mainly volatile material and the second peak consisted of mainly solid material. 
Bernemyr et al. invested the influence of dilution on the characteristics of tailpipe exhaust particle sizing with and without a Continuously Regenerating Trap (CRT) [115]. The exhaust was directly drawn from the tailpipe then diluted by a rotating disc diluter and analyzed by a volatility tandem Differential Mobility Analyzer (DMA) (v-TDMA, an instrument gives particle size distributions of unheated and heated aerosol sample). The CRT reduced the number of particles by $85 \%$ to $99 \%$ with different engine running conditions. However, the use of the CRT did not reduce the particle number in the sub-20 $\mathrm{nm}$ range. The particle sizing without the CRT was not as sensitive to the dilution ratios as the sizing with the CRT. While equipped with the CRT, higher dilution ratios or heating were likely to suppress the particle nuclei mode formation but had little effect on the accumulation mode. Further investigation on the fuel sulfur content suggested that higher sulfur content fuel (400ppm) would largely increase the particle number of nuclei mode compared to the $3.5 \mathrm{ppm}$ sulfur content fuel [42].

\subsection{Dispersion Modeling}

The near- and far-field plumes from internal combustion engines or other chemical reaction fields differ significantly. For the exhaust plume from a truck on highway, the plume process may be only significant for seconds before the pollutants are dispersed by air turbulence. Under such circumstances, only the near-field plume development needs to be considered for investigating aerosol size distribution evolution. For the exhaust plume from a ship or an aircraft, the dispersion process may last for minutes. This allows for more time for the oxidation of pollutants and aerosol aging. The plume from a large open explosion or forest fire burn may have a diameter of hundreds of meters and be slowly diluted over hours or days depending on the atmospheric stability. Once the exhaust plume is dispersed in the atmosphere, the PM composition and size continues to evolve in the atmosphere, but far more slowly compared to the PM inside a visible plume. For all kinds of plumes, the dilution rate over time and space is always the key variable to look into to predict the dispersion process [116].

To predict the pollutant dispersion in the atmosphere, empirical, analytical and numerical models were built over years to predict the concentration variation in a plume 
dispersion process. Dispersion models not only provide approaches for ambient DPM concentration evaluation as well as secondary pollutants quantification, but also are promising methods to distinguish on-highway and non-road DPM contributions to the ambient PM. Those models can be categorized as empirical Gaussian models, Lagrangian model, similarity models, probability density function models, statistical models, $k-\varepsilon$ models, large eddy simulation models, meandering plume models and second order closure models [117]. Each of those models has its own assumptions, limitations, hence its own focus. Among those models, the method of CFD simulation is becoming a promising technology for short-range pollutant dispersion modeling. Project Prairie Grass (PPG) data and Mock Urban Setting Test (MUST) data were widely used in dispersion modeling [118]. The PPG study was a classic field diffusion experiment performed in 1956. A total of 68 runs of sampling were performed by releasing tracer gas $\mathrm{SO}_{2}$ in an open agricultural field and measuring on arcs at different distances. The MUST study was a large outdoor field study conducted in 2001 for urban dispersion model development and validation. The propylene was used as the tracer gas and a 12 by 10 array of cargo shipping containers in a 0.5 mile square were used as obstacle arrays [119].

The Gaussian plume models are the most widely used dispersion modeling approaches. They predict the pollutant dispersion by analytically solving the Eulerian advection-diffusion equation. A steady state continuous emission and the normal distribution of lateral and vertical concentration profiles are assumed [120]. Gaussian models have limited applications under idealized meteorological conditions. Gautam et al. showed that the plume concentrations predicted by the Gaussian model were correlated well with measured concentrations in the far field region after adjusting the diffusion coefficients according to ambient conditions [121].

Cass et al. estimated the source contributions of ambient PM concentration with a Lagrangian particle-in-cell model [109]. The model indicated that 1982 annual DPM concentrations ranged from $1.9 \mu \mathrm{g} / \mathrm{m}^{3}$ to $5.6 \mu \mathrm{g} / \mathrm{m}^{3}$ in the Los Angeles area. It also predicted that HD trucks contributed $66 \%$ of the ambient DPM for southern California. Kleeman and Cass remodeled a Lagrangian model to predict aerosols' secondary 
transportation and transformation [122]. The modeling results were compared with PM measurement data in Claremont, CA, on August 27-28, 1987. The PM10, EC, OC concentration levels and the size distribution of the aerosols were reasonably simulated.

Hyvönen et al. used data mining approaches to model factors influencing aerosol formation during dispersion [123]. A dataset of 8 years of sampling of atmospheric aerosol at the boreal forest station in Southern Finland was analyzed using statistical models including clustering and classification. A total of 80 variables such as radiation, humidity, $\mathrm{SO}_{2}$ level, ozone level, and present aerosol surface area were collected during the study. The data mining and modeling results indicated that two key factors, relative humidity and condensation sink (pre-existing aerosol particle surface), explained $88 \%$ of the aerosol nucleation occurrences which only appeared with low relative humidity and condensation sink values.

Wei et al. applied steady-state Reynolds-averaged Navier-Stokes (RANS) Equations with the $k-\varepsilon$ model to test the PPG and MUST datasets [124]. The simulation results demonstrated that the $k-\varepsilon$ model can simulate the atmosphericsimilar boundary layer and the plume dispersion over an open field with and without building obstacles well.

Five independent investigation groups (CFD Research Corporation, GexCon, Lawrence Livermore National Laboratory, George Mason University, and Air Resources Laboratory) applied five different CFD models (CFD-Urban, FLACS, FEM3MP, FEFLO-Urban, and Fluent-Urban) in 2006 to simulate dispersion of chemical of biological agents released into the atmosphere in large cities [125]. The same atmospheric boundary layer conditions measured were applied to those five CFD models. The simulated wind flow patterns of five models showed good agreement with each other and with the measure winds during a field experiment.

CFD simulations were also widely used to model the aerosol dynamics inside the exhaust plume after the engine tailpipe. Kim et al. developed a CFD model to predict PM 
formation via nucleation and coagulation inside the exhaust plume from on-road HDDE vehicles [126]. The simulations were conducted with CFD software Fluent and it was predicted that an increase of relative humidity from $10 \%$ to $90 \%$ would decrease the nucleus diameter of the particles by about $30 \%$ and increase the number concentration by sixfold with a 50ppm sulfur content fuel. The simulated values of PM size distribution and concentrations were validated with a wind tunnel experiment.

Jiang et al. performed CFD simulations to evaluate the tailpipe exhaust dispersion of a Dodge vehicle by combining an aerosol dynamic model with a three dimensional CFD software tool Banff [127]. The study examined how exhaust plume streamlines, ambient particle size distributions and vehicle and wind speed affect the particle size distributions in the exhaust plume. The results showed the vehicle and wind speed had large effects on the particle size distributions of the exhaust plume. The study also demonstrated that the ambient air particles affected the larger sizes of the exhaust plume. However, less than 5\% difference from the particle size distributions at the same distance of different streamlines in the exhaust plume were observed from this study.

Uhrer et al. conducted a CFD simulation study of dilution and aerosol dynamics within a diesel car exhaust plume and compared the simulation results with on-road measurement [128]. A physical model for particle formation and growth in the diesel exhaust plume was developed and this model was coupled with the Fine Particle Model (CFM). Fluent was set up to solve the RANS equations with modeled parameters. The simulated results for temperature, humidity and $\mathrm{CO}_{2}$ agreed fairly well with the measurements. The particle nucleation concentrations and growth were also predicted with the simulations. The simulations showed that the highest nucleation rates occurred within areas of strongest gradients of temperature, humidity, $\mathrm{H}_{2} \mathrm{SO}_{4}$ level and dilution ratio. The simulation results also indicated that organic species with very low vapor pressures may cause the growth of nucleation model particles within about one meter beyond the end of the tailpipe. 


\section{EXPERIMENTAL SETUP AND METHODOLOGY}

\subsection{Experimental Equipment}

\subsubsection{Cambustion DMS500 Fast Particulate Spectrometer}

Particle size distributions were measured using a Cambustion DMS500 Fast Particulate Spectrometer (Figure 5) [129-134], which is capable of measuring particle size distributions in multiple size bins ranging from $5 \mathrm{~nm}$ to $1000 \mathrm{~nm}$ in real time.

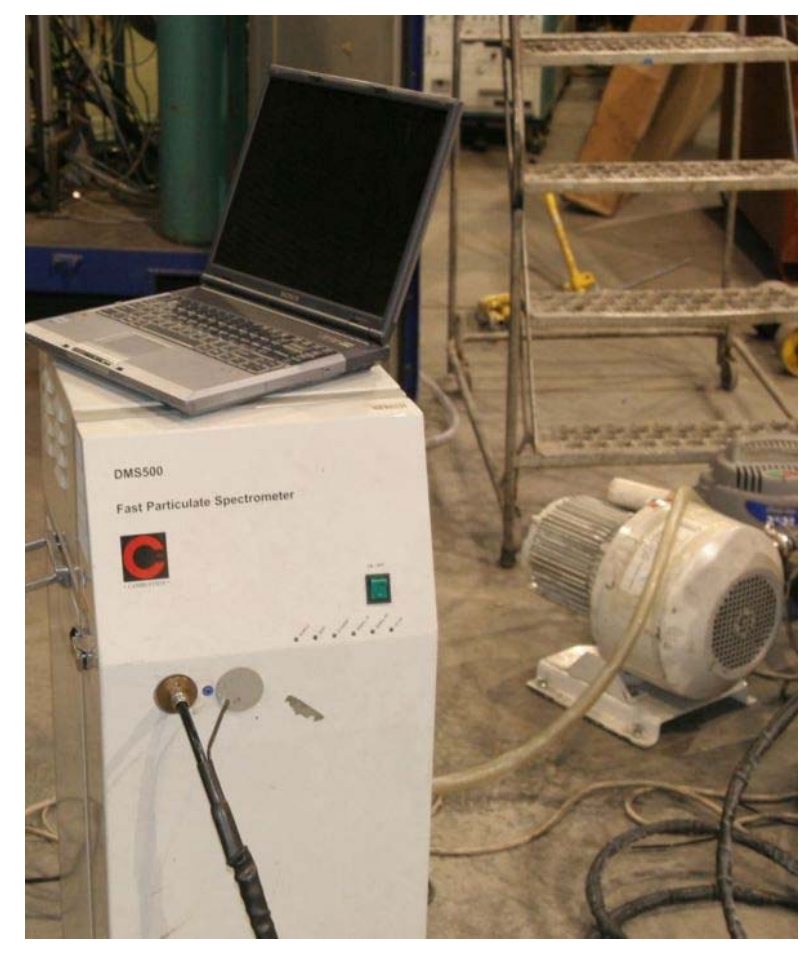

Figure 5 View of DMS500 with vacuum pump

The instrument estimates particle size distribution based upon electrical charge mobility principles. Specifically, particles are electrically charged by a high voltage corona discharger and enter a classification column with a central high voltage electrode rod as shown in Figure 6. The particles travel along the column and are deflected toward outer electrometer rings. Electrically charged particles with lower electrical mobility travel further along the column. The electrical mobility of charged particles, measured as electrical current as the particles arrive on electrometer rings, is a function of particle 
size. The instrument software converts the measured ring currents into the particle size distribution using an inversion matrix.

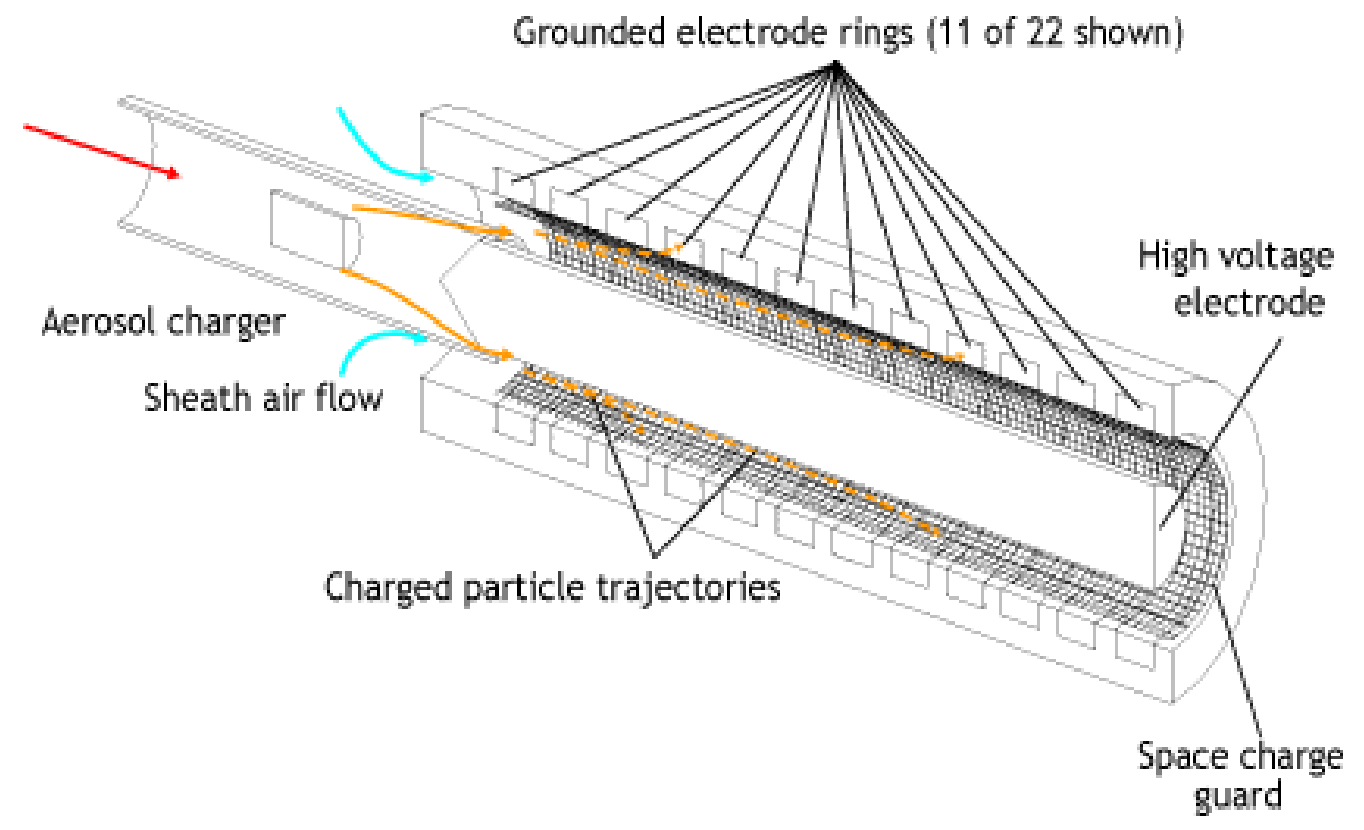

Figure 6 Operational principal of DMS500 [129]

The DMS500 fast particulate spectrometer was the key instrument used in this research to obtain the PM particle size distributions. The following description of the DMS500 particulate spectrometer can be found on http://www.cambustion.com: "The Cambustion DMS500 offers measurement of the particle size distribution from $5 \mathrm{~nm}$ to up to $2.5 \mu \mathrm{m}$. Special patented features of the differential mobility classifier give this particle sizer the fastest time response available in any ultrafine aerosol measurement. The time response is especially useful for investigation of fast changing spectra such as combustion engine emissions, drug delivery systems such as inhalers, and roadside ambient aerosol sampling. But it also dramatically increases the efficiency and convenience of experimental measurements compared with scanning instruments. The DMS500 is fully controlled by a computer up to $200 \mathrm{~m}$ from the instrument. The interface includes data logging of the spectrum and up to four auxiliary analogue signals for correlation with other measurements. Four analogue outputs can be configured to reflect the particle concentration in any desired size range. For integration with other test equipment, the instrument can be controlled by a digital remote control and it can even trigger other equipment at a preset aerosol concentration (for example a roadside 
camera). Data analysis tools are included to ease post-processing in Microsoft Excel [135]."

\subsubsection{Portable Particulate Measurement Device}

The PPMD was a device with built-in proportional diluters to collect electrically charged Diesel particles on an array of eight QCM crystal plates. The PPMD was also equipped with a exhaust flow meter to measure the raw exhaust flow rate in real time. The internal components of the PPMD are shown in Figure 7.

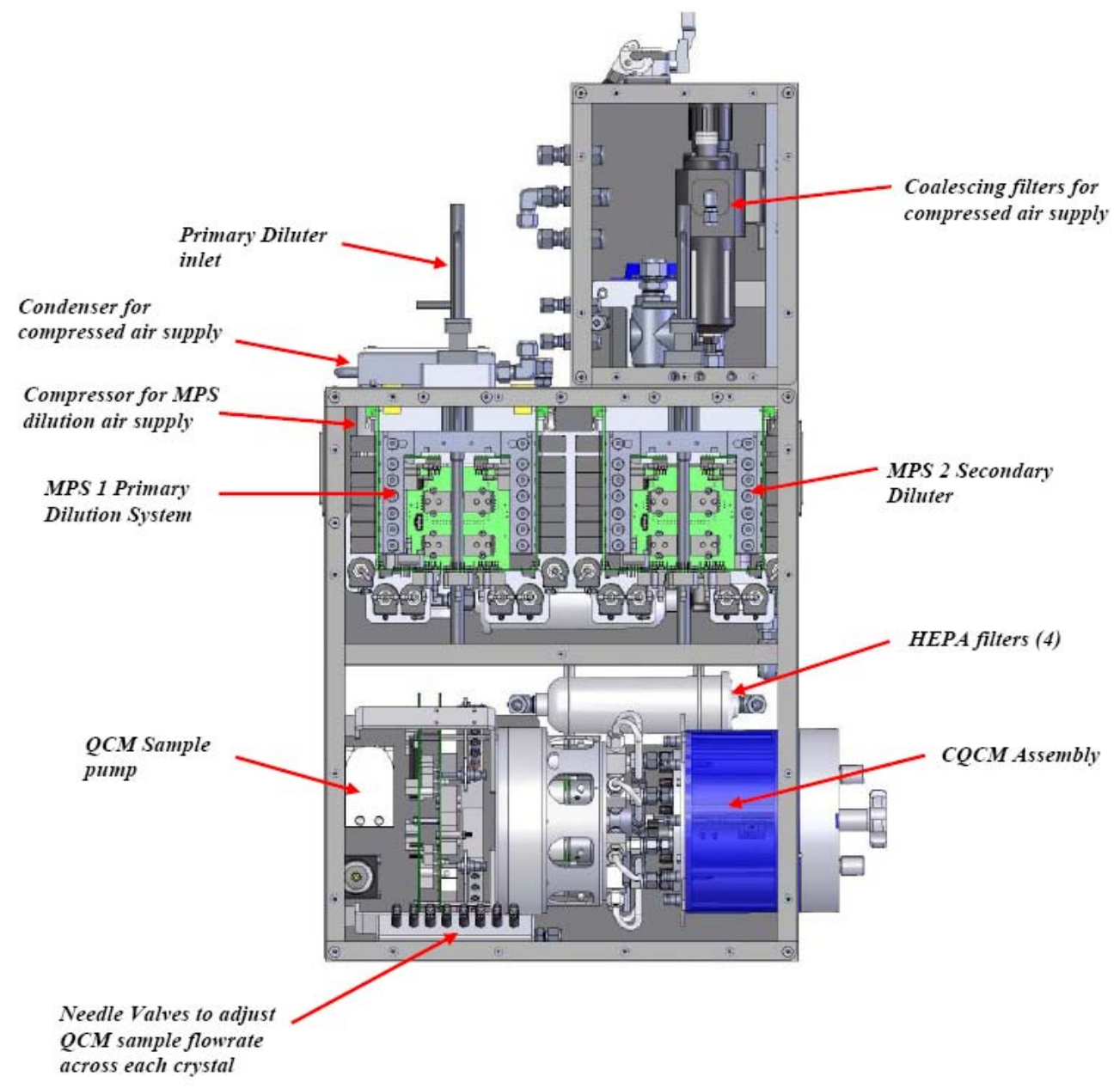

Figure 7 Internal components of PPMD [136]

Since previous measurement experience using the QCM part of the PPMD indicated low measurement reliability and repeatability, the mass measurement part of the PPMD was not used in this research and was not discussed. The diluters of the PPMD were employed to provide accurately controlled dilution ratios. Two identical MPS 
diluters functioned as the diluters of the PPMD and could be configure as a single or a two stages diluter. The operational principal of the MPS diluter is shown in Figure 8.

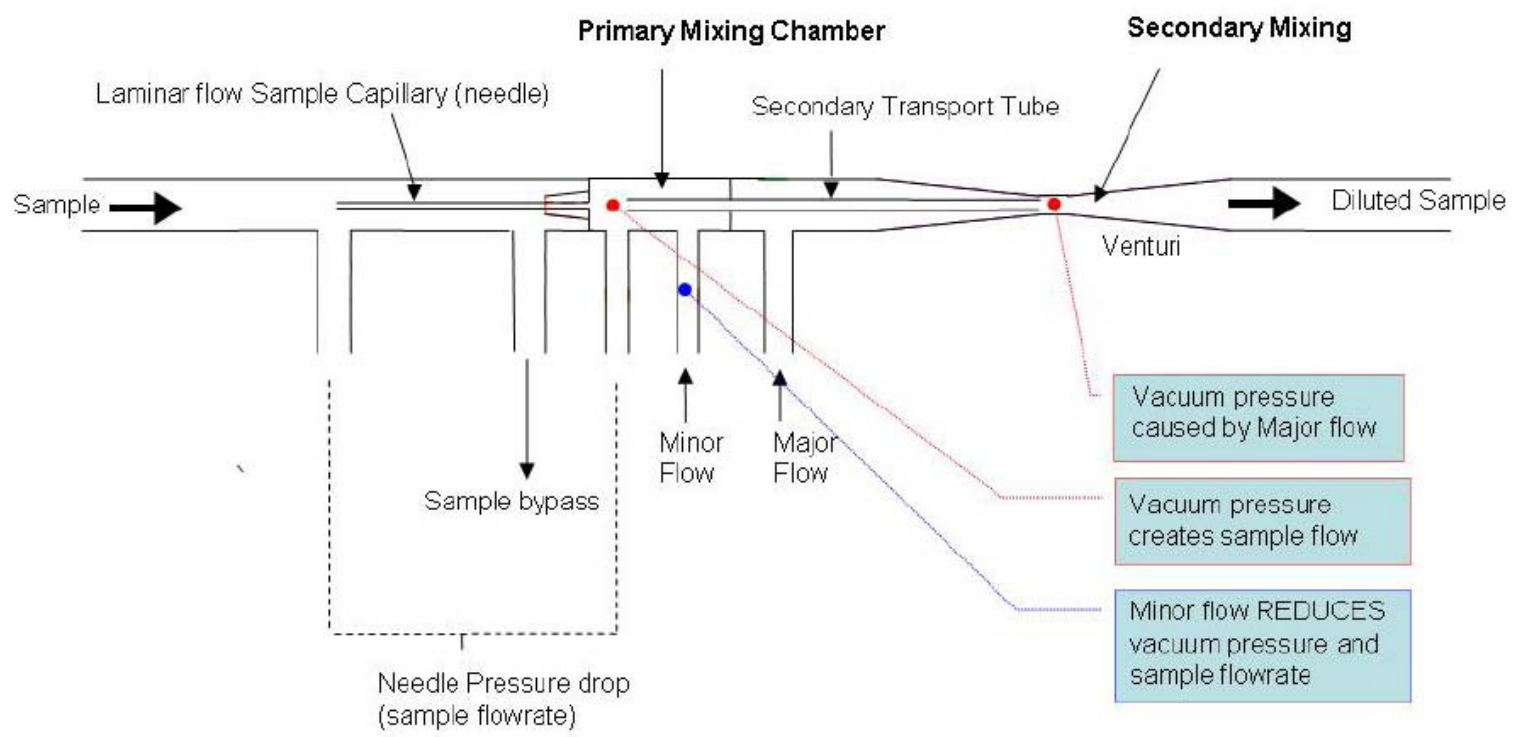

Figure 8 Cross-section diagram of the MPS diluter [136]

The following description illustrates how the diluter worked: "The exhaust sample is extracted through a laminar flow capillary and immediately mixed with the primary (MAJOR) dilution flow. This diluted exhaust then flows through a secondary transport tube to the throat of the venturi where it mixes with the secondary (MINOR) dilution air. The MAJOR dilution flow rate passing through the venturi creates the suction needed to draw the sample. This allows a sample to be extracted and the diluted sample to be delivered at or near atmospheric pressure" [136]. The dilution air provided for the PPMD was high efficiency particulate air (HEPA) filtered shop air and the pressure was regulated inside the PPMD. The flow rates of the primary and secondary dilution air were controlled by two banks of seven solenoids and critical orifices of different sizes. A TSI Series 4100 reference flow meter was used for calibration of dilution flows and sample flow.

\subsubsection{Horiba MEXA-7200D Motor Exhaust Gas Analyzer}

The Horiba MEXA-7200D motor exhaust gas analyzer (Figure 9) was equipped for the 2007 mobile laboratory and it was used to measure gaseous emissions levels in 
this research for determination of exhaust dilution ratios. The current configuration of MEXA-7200D can be used to measure emissions including Total Hydrocarbons (THC), $\mathrm{CO} / \mathrm{CO}_{2}$, and $\mathrm{NO}_{\mathrm{x}}$. It is composed of eight basic units: the gas divider, the main control unit (MCU), the interface unit (IFC), the analyzer rack (ANR), the power supply unit (PSU), the solenoid valve unit (SVS), the sample handling unit (SHS) and the OVN-700 module. The MCU is based on a personal computer (PC) architecture running the Linux RedHat as the operating system. The software of Horiba MEXA-7200D on the MCU controls and monitors all other modules of MEXA-7200D and can be operated locally as well as be controlled remotely over a laboratory host PC. The IFC is a networking device providing communication between all other modules of the MEXA-7200D. The ANR can be fitted with a maximum of 5 analyzer modules [137].

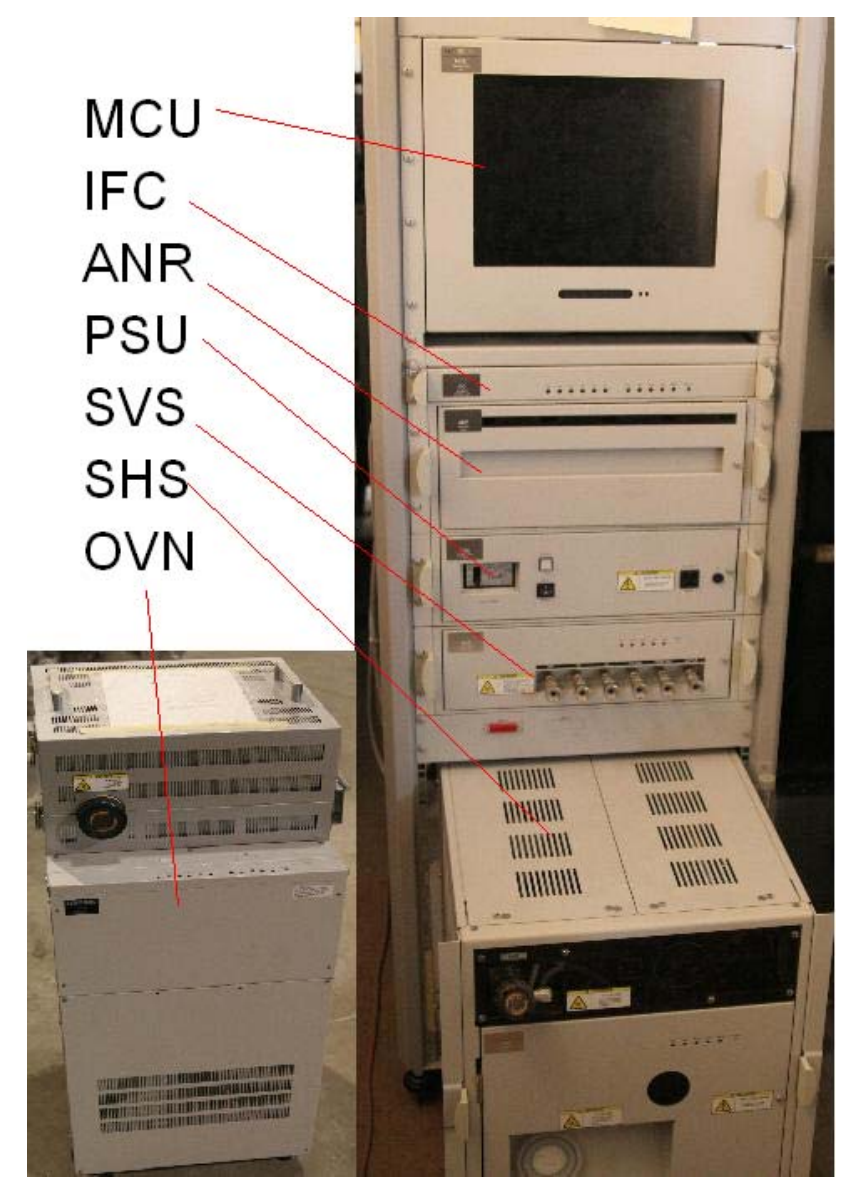

Figure 9 Horiba MEXA-7200D

The analyzer modules can be installed to and removed from the ANR without using any tools with a connection panel. Currently, one AIA-721A CO analyzer and one AIA-722 $\mathrm{CO} / \mathrm{CO}_{2}$ analyzer are installed on the ANR. The AIA-721A CO analyzer can 
measure $\mathrm{CO}$ emissions over two setting ranges: 50-2,500ppm and 100-5,000ppm. The AIA-722 CO/ $\mathrm{CO}_{2}$ analyzer measures $\mathrm{CO}$ levels over $0.5-12$ volume percent (vol \%) and $\mathrm{CO}_{2}$ levels over 3-20 vol \%. The SVS controls the operational and calibration gases to the analyzer modules. The SHS filters, conditions and pumps the exhaust sample gas to the analyzer modules. The SHS unit is located on a standalone cart and moved into the bottom section of MEXA-7200D rack. The OVN-700 module combines a FIA-725A THC analyzer and a CLA-720MA $\mathrm{NO}_{\mathrm{x}}$ analyzer as a joint solution for analyzers operated under sample-heated conditions. The FIA-725A THC analyzer measures THC emission over 10-50,000ppm and the CLA-720MA $\mathrm{NO}_{\mathrm{x}}$ analyzer measures $\mathrm{NO}_{\mathrm{x}}$ emission over 1010,000ppm. Gas analyzers in Mexa-7200D were calibrated to ranges shown in Table 2.

Table 2 Calibration setting of MEXA-7200D gas analyzer

\begin{tabular}{|l|l|l|}
\hline & Range & Span \\
\hline $\mathrm{CO}(\mathrm{L})$ & $250 \mathrm{ppm}$ & $100 \mathrm{ppm}$ \\
\hline $\mathrm{CO}_{2}$ & $12 \%$ & $3 \%$ \\
\hline $\mathrm{HC}$ & $500 \mathrm{ppm}$ & $300.9 \mathrm{ppm}$ \\
\hline $\mathrm{NO}_{\mathrm{x}}$ & $1000 \mathrm{ppm}$ & $505 \mathrm{ppm}$ \\
\hline
\end{tabular}

\subsubsection{Engine and test cell}

This study was conducted on a naturally aspirated, 2.4L, 40kW ISUZU C240 diesel engine equipped with a Cleaire ${ }^{\circledR}$ DPF. Previous studies have reported PM mass concentration levels at or lower than 2007 standard levels can be achieved by running this engine with a DPF and ULSD fuel [138]. As a primary indirect injection engine, its relatively high $\mathrm{NO}_{\mathrm{x}}$ emission levels in raw exhaust make it easier to determine dilution ratios inside the primary tunnel by measuring $\mathrm{NO}_{\mathrm{x}}$ concentration levels. Because this study was not aimed at transient PM characteristics performance of an engine, the engine was operated at steady state conditions of $1800 \mathrm{rpm}$ at load settings of 102 and $122 \mathrm{Nm}$. The fuel used for this study was ULSD with $6 \mathrm{ppm}$ sulfur content. The ISUZU C240 diesel engine was connected to a Mustang eddy current dynamometer. The engine's and 
dynamometer's running conditions were set by a digital throttle controller (DyneSystems DTC-1) and a digital dynamometer controller (DyneSystems DynLocIV). A Meriam 50MC2-2 laminar flow element (LFE) was used to measure the engine intake air flow rate [139]. The diesel fuel used for this research was held in a fuel barrel and weighed with a digital scale during the test. The weight changes were recorded during the test for the calculation of total exhaust flow rate based on intake air flow rate and fuel consumption rate. The engine test cell setup is shown in Figure 10.

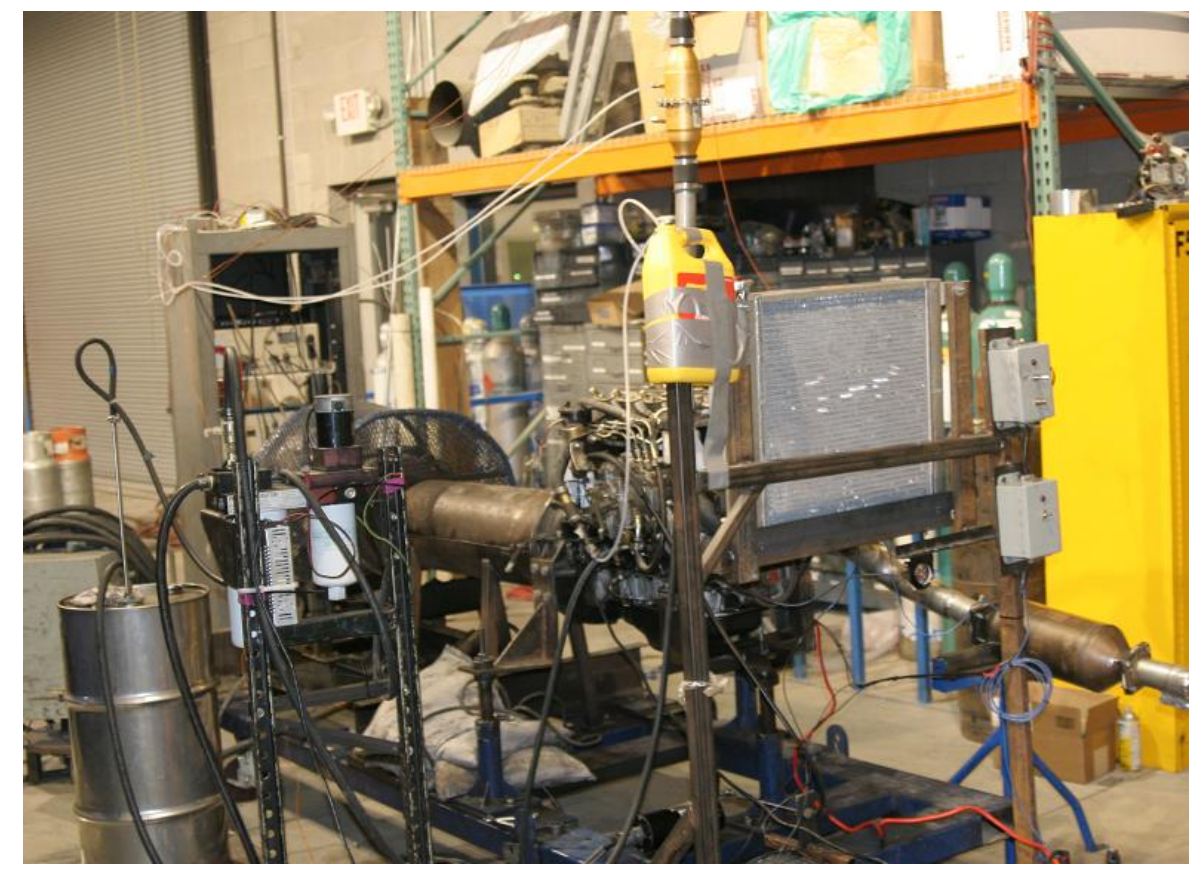

Figure 10 Engine test cell setup

\subsubsection{Data Acquisition (DAQ) System}

A DAQ system for this research was prepared to collect data. A NI SC-2345 ${ }^{\circledR}$ shielded carrier was used to house NI SCC signal conditioning modules. Those SCC modules were used to collect signals including engine speed, torque, coolant temperature, exhaust temperature, intake air temperature, intake air humidity, LFE differential and absolute pressures, and the DPF differential pressure. All the signals conditioned on the SC-2345 board were sent to a NI 6062E DAQ PC-MCIA card on a laptop computer for further processing. The DAQ software running on the laptop computer was written in NILabVIEW $^{\circledR}$ with the option to save data in different frequencies. Figure 11 shows the 
view of the DAQ board NI SC-2345 shielded carrier with corresponding modules installed. Figure 12 shows the partial view of program diagram of the DAQ software in LabVIEW $^{\circledR}$.

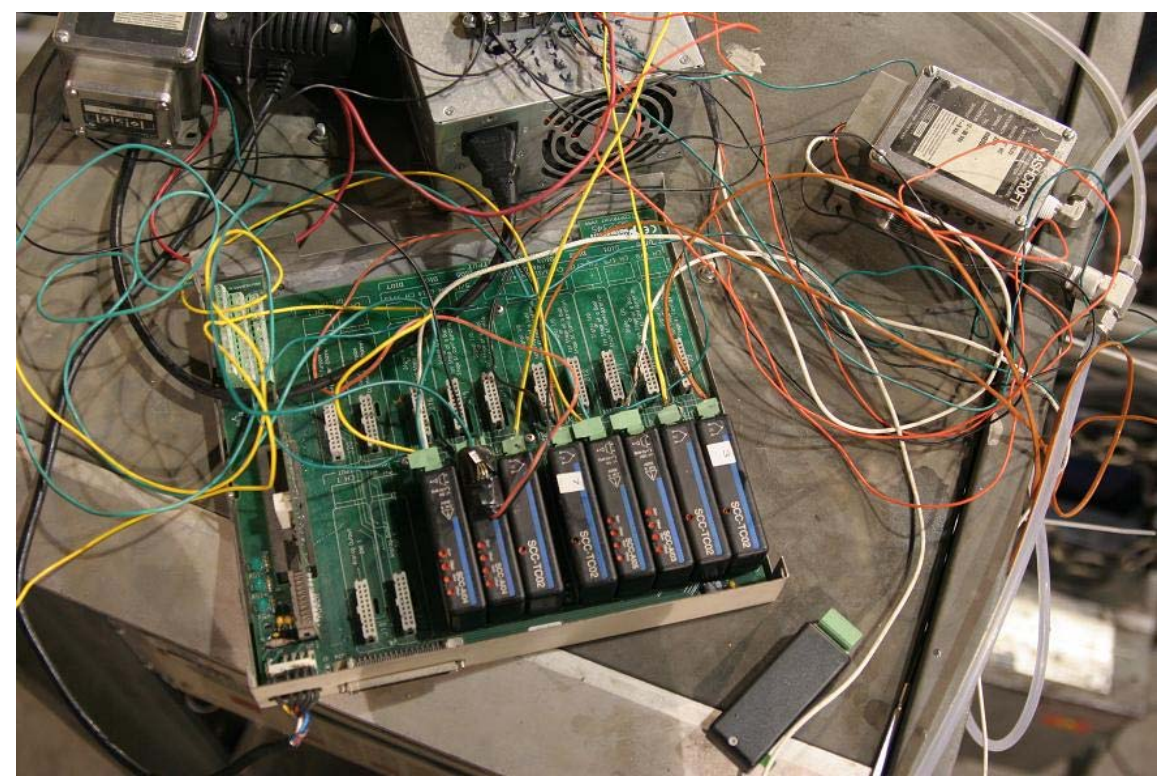

Figure 11 View of DAQ board and module wiring

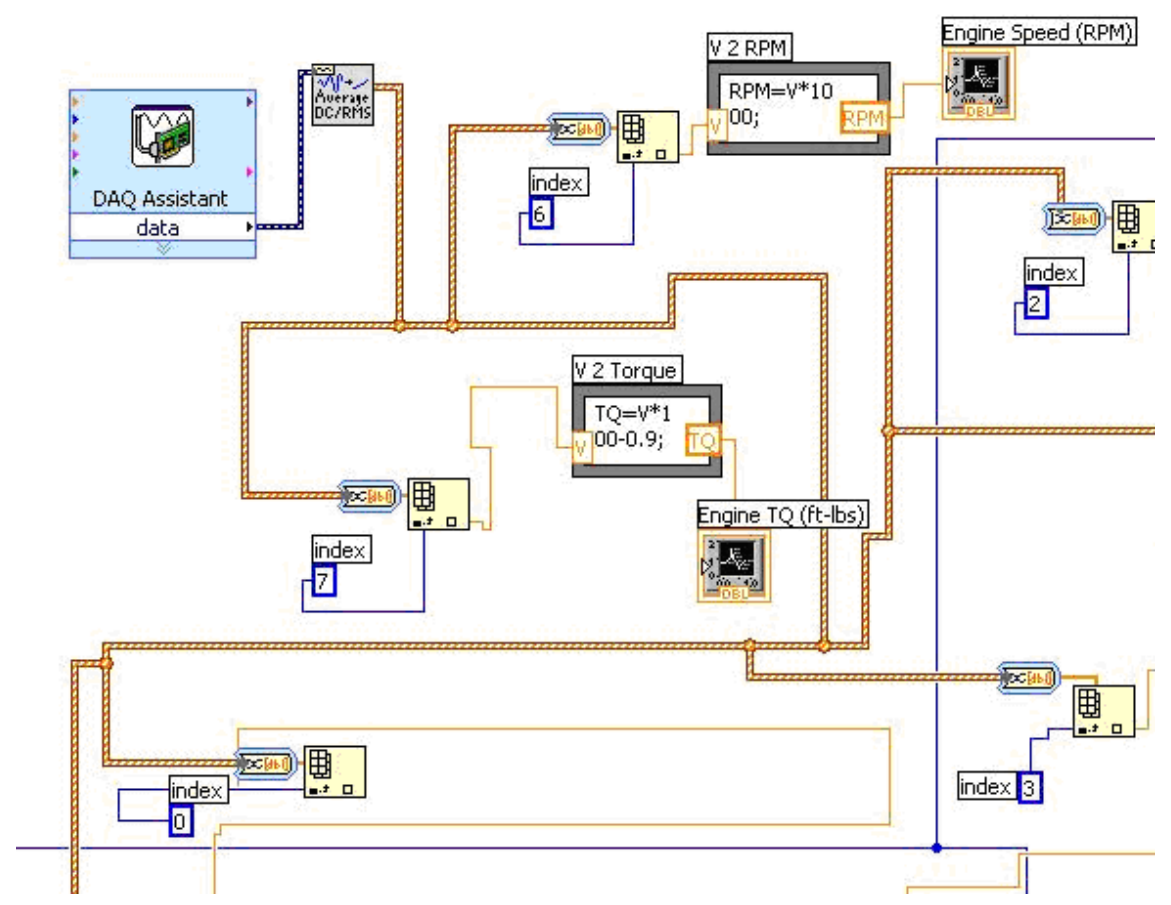

Figure 12 Partial view of the DAQ software program diagram in LabVIEW ${ }^{\circledR}$ 


\subsubsection{Emissions Test Tunnels}

WVU CAFEE researchers have designed and built a transportable (or mobile) low emissions measurement laboratory which is compliant with CFR Part 1065 in terms of emissions sampling and measurement (The system is not fully compliant for dlution air conditioning and/or proportional bag flow at the time of this dissertation). The engine test procedures defined in CFR Part 1065 apply to wide categories of engines or vehicles of new model years (the term "new" is defined in corresponding CFR parts) [34]. The new WVU mobile emissions laboratory was motivated by the need to quantify and characterize emissions at reduced exhaust concentrations and to provide a tool for benchmarking on-board emissions measurement systems.

Figure 13 shows the 2007 emission test tunnels which were used for the phase II test. The emission test tunnels follow the concept described in Figure 2 but with the capability of measuring relatively clean engine (for example CNG engine) emissions or dirty conventional engine (diesel engine) emission with specifically assigned tunnels. The top tunnel is used for the clean engine emissions test and the middle tunnel is used for the dirty engine emissions test. The dirty diesel tunnel was used in this research. While the test of diesel engine emissions was going on, the end of the diesel tunnel was connected to the bottom tunnel connected to blower. The exhaust inlet and the end of CNG tunnel is sealed during the diesel emissions test.

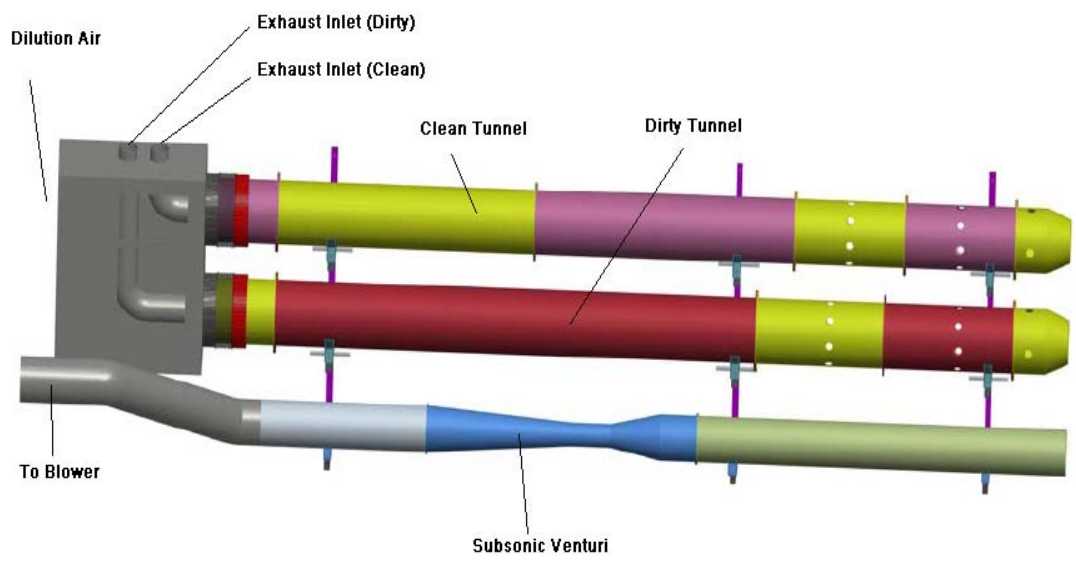

Figure 13 Schematic of laboratory emission test tunnels used for testing 
One important mission of this dissertation is to provide detailed description of the 2007 mobile laboratory trailer. The next chapter is dedicated to discuss the 2007 mobile laboratory. Figure 14 shows the side view of the 2007 mobile laboratory (without the view of the chassis dynamometer).

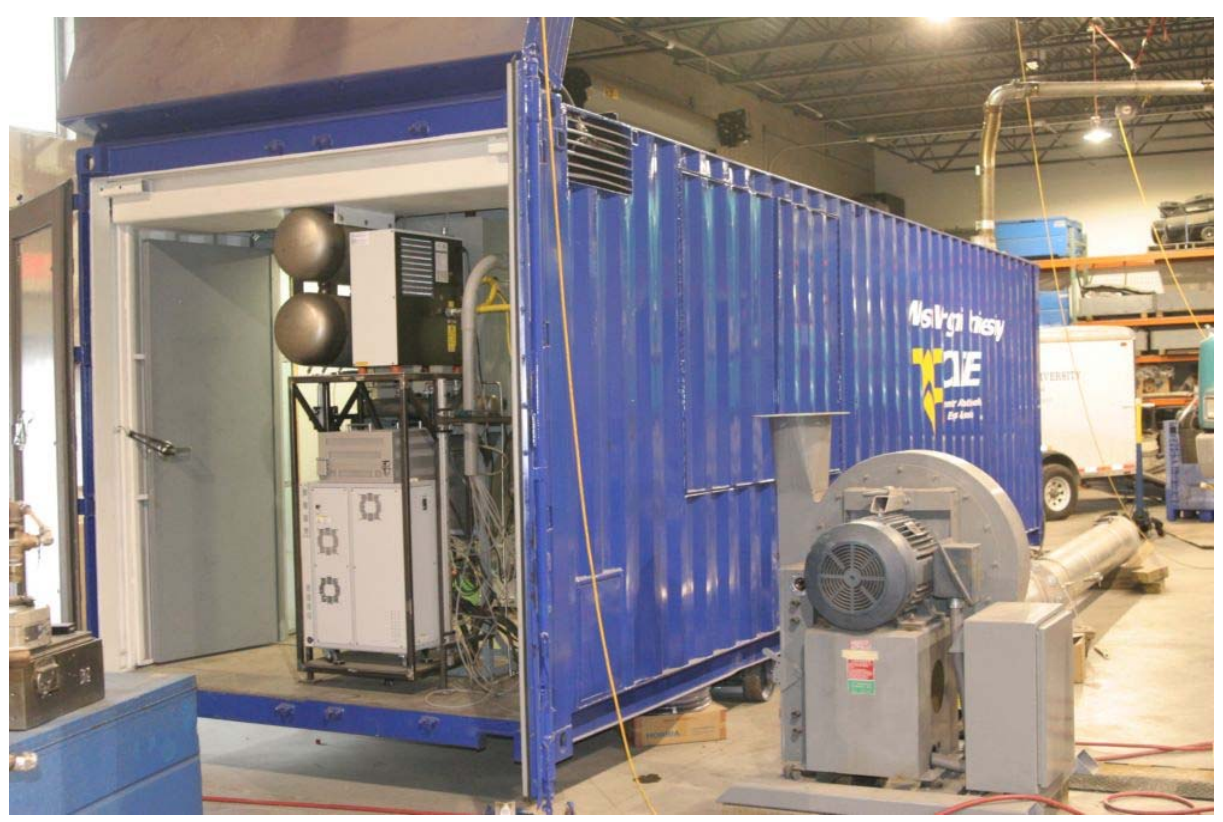

Figure 14 Side View of the 2007 mobile laboratory

\subsection{Experimental Setup}

\subsubsection{Tunnel Test Setup}

The dilution tunnel used in this study was part of the transportable dual primary full-flow dilution tunnel emissions measurement laboratory newly designed and constructed by West Virginia University's (WVU) Center for Alternative Fuels, Engines, and Emissions (CAFEE). This $9.14 \mathrm{~m}$ long cargo container was constructed to house a portable emissions measurement facility, comprised of a HEPA filter for the primary exhaust dilution air, two primary full-flow dilution tunnels, a subsonic venturi with variable speed blower, a secondary particulate matter sampling system, a gaseous emissions analytical bench instrumentation system, a computer based data acquisition and control system, full air conditioning and ventilation system, and chassis dynamometer control systems. Two tunnels, $0.46 \mathrm{~m} \mathrm{ID}$ and $6.1 \mathrm{~m}$ long, were designed to provide dedicated measurement capability for both low PM vehicles and legacy diesel- 
fueled vehicles. This provision reduced tunnel history effects between test programs which address differing exhaust composition and PM loading: it is known that PM hangup from prior tests can mar accurate PM measurement from low emissions vehicles. CFR Part 1065 allows for the use of a full flow CVS tunnel with a secondary dilution tunnel for PM sampling. The two stage dilution system of a full flow primary tunnel has the advantage of controlling the filter face temperature more precisely, compared to a single-stage dilution tunnel. Also, to achieve filter face temperature requirement $\left(47^{\circ} \mathrm{C} \pm\right.$ $5^{\circ} \mathrm{C}$ ) with a full flow tunnel alone would require the use of a substantially larger tunnel, with a larger venturi and blower to establish adequate flow rates. The single tunnel would necessitate a high dilution ratio that would result in gaseous exhaust species concentrations that would be difficult to quantify accurately. When a primary and secondary tunnel is used, the gaseous species are less diluted at the sampling plane than the particulate matter stream at the filter, because the gaseous species experience only the primary dilution prior to sampling.

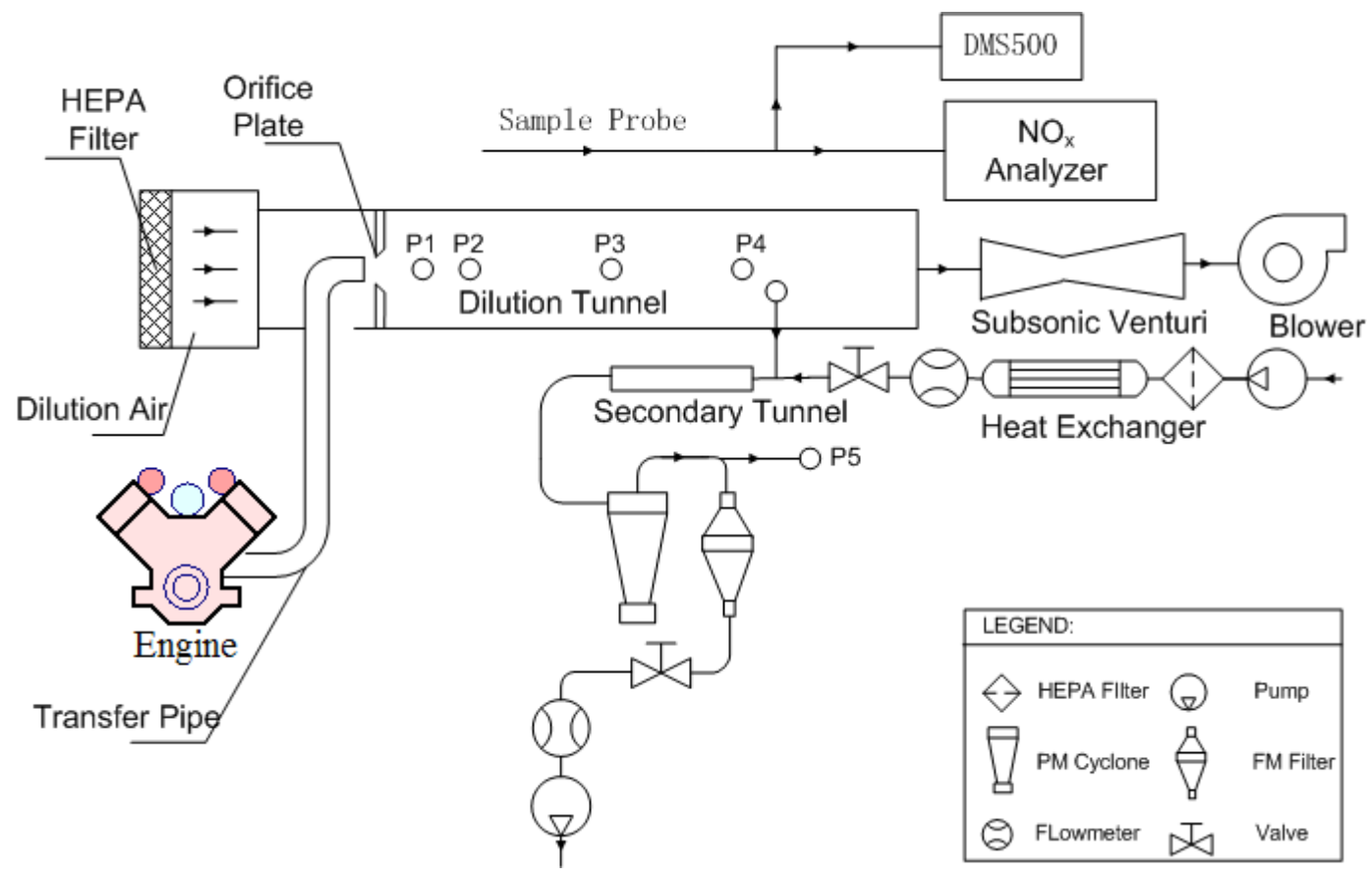

Figure 15 Schematic of tunnel test configuration

The laboratory grade analytical system could be transported to virtually any location with a demand for emissions testing, either with or without WVU's transportable 
medium or heavy duty chassis dynamometers. Alternatively, the system can be loaded onto a flatbed trailer in order to test emissions while a vehicle capable of towing the trailer is operated over the road [140]. A schematic of system configurations for this study is shown in Figure 15. Four sampling ports (noted as P1 through P4) were tapped with Swagelok ${ }^{\circledR}$ fittings on the primary tunnel for access of the sampling probe. An additional sample port (noted as P5) was located after the PM cyclone and upstream of the PM filter holder.

During testing, ports that were not used for sampling were capped to prevent system leaks. One $0.5 \mathrm{~m}$ in length, $9.5 \mathrm{~mm}$ OD sampling probe was used to obtain the PM sample by inserting it into the tunnel and spanning the cross section. The sample was then drawn into the DMS500 and CLA-720MA $\mathrm{NO}_{\mathrm{x}}$ analyzer for measurements of size distributions and $\mathrm{NO}_{\mathrm{x}}$ concentration levels. An overall dilution ratio was assumed to be achieved where the flow was fully developed and mixed. The overall dilution ratio was calculated with the maintained constant primary tunnel flow rate and the engine exhaust flow rate. The engine exhaust flow rate was estimated by the summation of engine intake air flow rate, measured by a LFE, and fuel consumption rate ignoring mass loss post cylinder rings. Since the engine was running under steady-state conditions, with a constant exhaust flow rate, and the primary flow rate was maintained constant, the ratio of measured dilute $\mathrm{NO}_{\mathrm{x}}$ concentration levels, the background $\mathrm{NO}_{\mathrm{x}}$ concentration levels, and the $\mathrm{NO}_{\mathrm{x}}$ concentration level of the raw exhaust could be used to obtain estimations of local dilution ratios at different sampling locations across the tunnel sections.

DPM size distributions inside the primary and secondary dilution tunnels, with different dilution ratios, were investigated in this study. The dilution ratios of the secondary dilution flows were obtained by maintaining total tunnel flow rate and dilution air flow rate with two mass flow controllers, as shown in Figure 15. The sample flow of the DMS500, extracted upstream of the filter holder, was also accounted for during secondary dilution ratio calculations. The capability of the secondary PM sampling system to achieve desired dilution ratios had been tested and verified, and the results have been published [140]. Verification of the system involved measuring THC concentration 
levels with a Horiba FIA-725A heated flame ionization detector (FID) HC analyzer at the outlet of the secondary tunnel while a sample flow with a known THC (propane) concentration level was diluted to various dilution ratios. Overall primary dilution ratios were achieved by setting different primary flow rates, while the engine was running at a steady state with a constant exhaust flow rate. The primary flow rates were established by varying the blower speed, which was controlled with feedback from pressure and temperature measurements in the subsonic venturi upstream of the blower.

Particle size distributions across P1-P4 sections were measured with the primary flow rate set at $0.378 \mathrm{~m}^{3} / \mathrm{s}$ and $0.944 \mathrm{~m}^{3} / \mathrm{s}$ (800 SCFM and $\left.2000 \mathrm{SCFM}\right)$. Data were collected from at least eight sampling locations spanning the tunnel cross-section, from the centerline to the outer wall. Sample location distribution was apportioned such that each location would represent an equal sample surface area, that is, the centerline sample zone surface area was equated with successive annular zones in a radially increasing fashion. Specifically, measurements were taken at 0.0, 8.6, 12.2, 15.0, 17.3, 19.3, 21.1 and $22.9 \mathrm{~cm}$ away from the tunnel centerline. Since the sample points equally divided the tunnel cross-section and the preliminary measurements suggest the flow to be axisymmetric, the measured size distributions were assumed to be representative of the part of flow passing the divided area where the sampling probe tip was located. However, excessive dilution ratio gradients between the first (section center) and second $(8.6 \mathrm{~cm}$ from center) sampling points were observed when the samples were taken at P1 and P2. In order to provide additional data regarding the impact of dilution ratio and particle size distributions, measurements at additional sampling points near the tunnel centerline were also performed at P1 and P2 tunnel locations.

Particle size distributions of diluted flow were also measured at the outlet of the secondary dilution tunnel and upstream of the filter holder, with different secondary dilution ratio settings. The engine was operated at $102 \mathrm{Nm}$ and $122 \mathrm{Nm}$, at $1800 \mathrm{rpm}$, with a $0.378 \mathrm{~m}^{3} / \mathrm{s}$ primary flow rate during these tests. The total secondary tunnel flow rate was maintained at $63.7 \mathrm{lpm}$, and the tunnel wall temperature was controlled at $47^{\circ} \mathrm{C}$. 
In order to determine the appropriate positions of sampling ports on the primary tunnel, a CFD model was developed to examine flow development and mixing inside the primary tunnel, under test conditions encountered in this study. The commercially available CFD software, Fluent (Canonsburg, PA), was used to investigate the diluted flow behaviors inside the tunnel. A 2-D, axisymmetric, $\mathrm{k}-\varepsilon$ viscous model, with standard wall functions for the turbulent flow simulation, was employed. With the employment of the standard wall function approach, the viscous sub-layer of the near wall region was not resolved and only the fully turbulent region of the flow was computed. This "standard wall functions" approach saved computational resources without compromising the results of the turbulent region. At the mean time, a species transport modeling option was also enabled and configured to predict $\mathrm{NO}_{\mathrm{x}}$ concentration levels inside the tunnel.

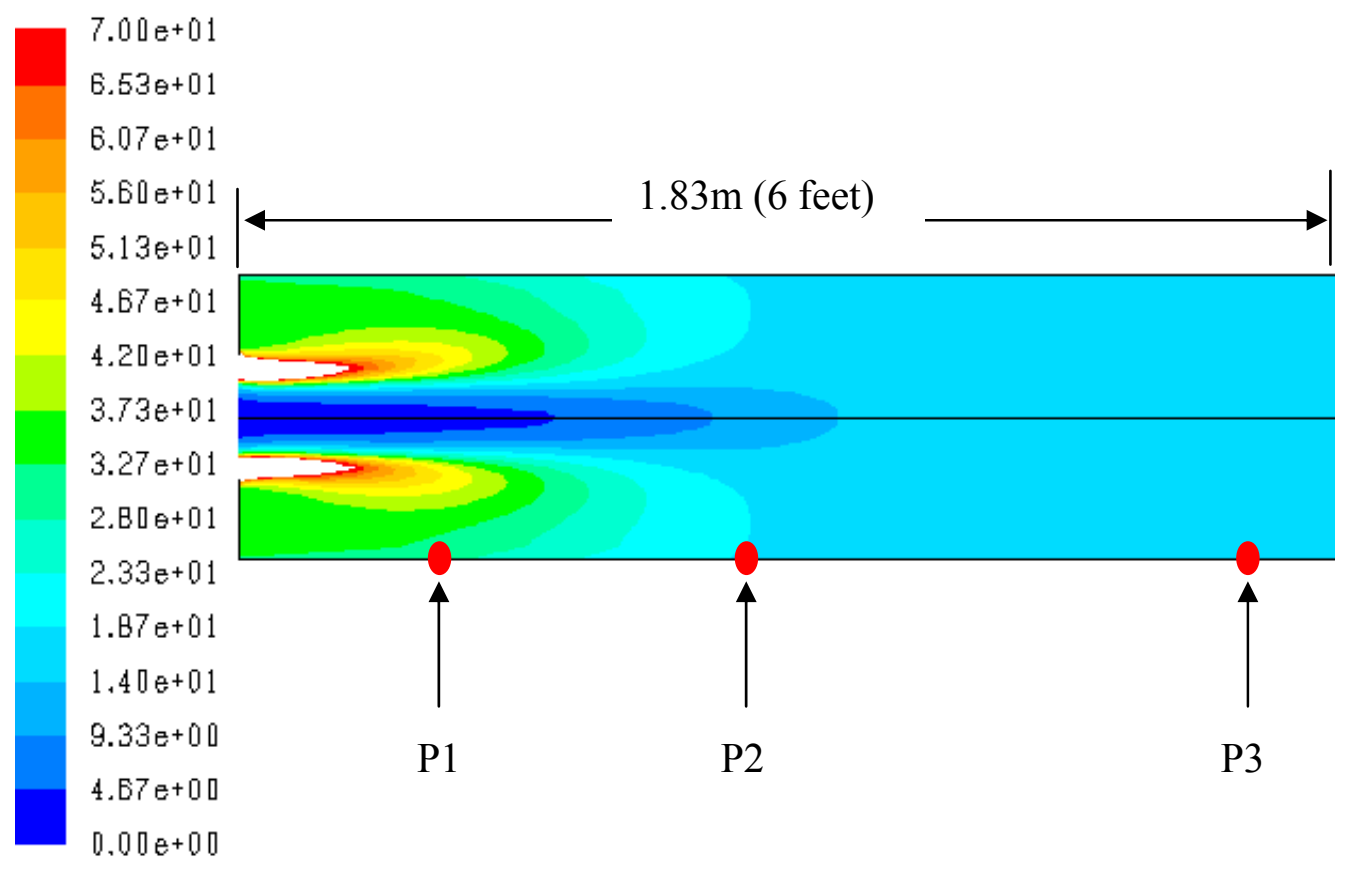

\section{Figure 16 Contour of the primary dilution ratios}

A fully mixed tunnel condition was assumed to exist when the measured minimum $\mathrm{NO}_{\mathrm{x}}$ concentration level across the tunnel section was at least $99.5 \%$ of the maximum $\mathrm{NO}_{\mathrm{x}}$ concentration level across the tunnel section. Figure 16 shows an example of the simulated dilution ratio contour within $1.83 \mathrm{~m}$ downstream of the orifice plate with P1 to P3 ports' locations. This contour graph was obtained at an engine operating condition of $102 \mathrm{Nm}$ torque output at a speed of $1800 \mathrm{rpm}$, with a primary flow rate 
setting of $0.472 \mathrm{~m}^{3} / \mathrm{s}(1000 \mathrm{SCFM})$ in the CFD model. The chemical component mass percentages of the raw exhaust were estimated by a chemical combustion balance equation and applied in the CFD model. The exhaust properties used for the CFD model are listed in Table A1 of the Appendix. For the estimation, dilution air $\mathrm{NO}_{\mathrm{x}}$ concentration levels were considered to be zero, while measured raw exhaust $\mathrm{NO}_{\mathrm{x}}$ concentrations of $500 \mathrm{ppm}$ were used. A simple custom field function, defined by dividing 500ppm by the simulated $\mathrm{NO}_{\mathrm{x}}$ concentration level values, was used to generate dilution ratio values in this model. The dilution ratio contour was obtained later during the post processing procedure.

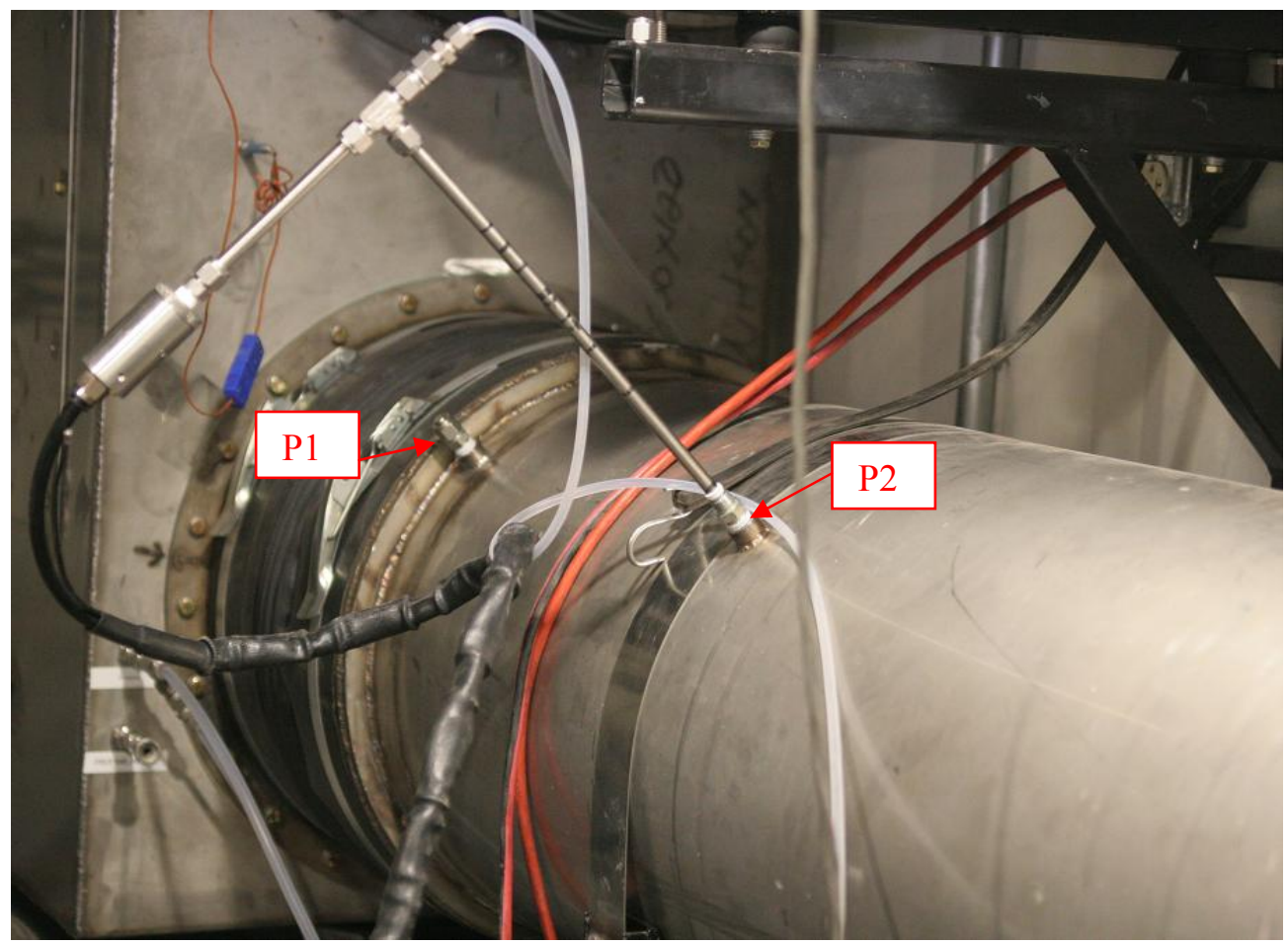

Figure 17 Sample ports $\mathrm{P} 1$ and $\mathrm{P} 2$ on the primary tunnel

A set of criteria to determine the locations of sampling ports was established by examining the CFD model results. The locations P1 and P2 were selected so that the flow was at its early and late stages of mixing across the respective sections. At these sections, flow recirculation was still present, therefore location P3 was selected to be at the point where the flow was just fully mixed and no more flow recirculation occurred. The location of P4 was selected as close as possible to the secondary dilution tunnel inlet in 
order to obtain information of particle size distributions before introduction into the secondary tunnel. Changes of size distribution, even in the presence of fully mixed flow, could then be investigated by comparing sizing measurements results at P3 and P4. Along with simulated dilution ratios and flow velocity profiles inside the primary tunnel, access ergonomics associated with sample port locations were also taken into consideration. Final locations of P1 through P4 were set as 0.30, 0.76, 1.68, and $4.57 \mathrm{~m}(1,2.5,5.5$, and 15 feet) downstream of the orifice plate. A picture of locations P1 and P2 on the primary tunnel is included below (Figure 17). The sample probe was installed in sample port P2 when the picture was taken.

Further verification that the CFD model was able to provide sufficient information for flow development and mixing was obtained by comparing measured and simulated dilution ratios across sample port sections of the tunnel. The simulated results shown in Figure 16 were compared to measured values in Figure 18 for locations at P1 and $\mathrm{P} 2$ across the tunnel sections.

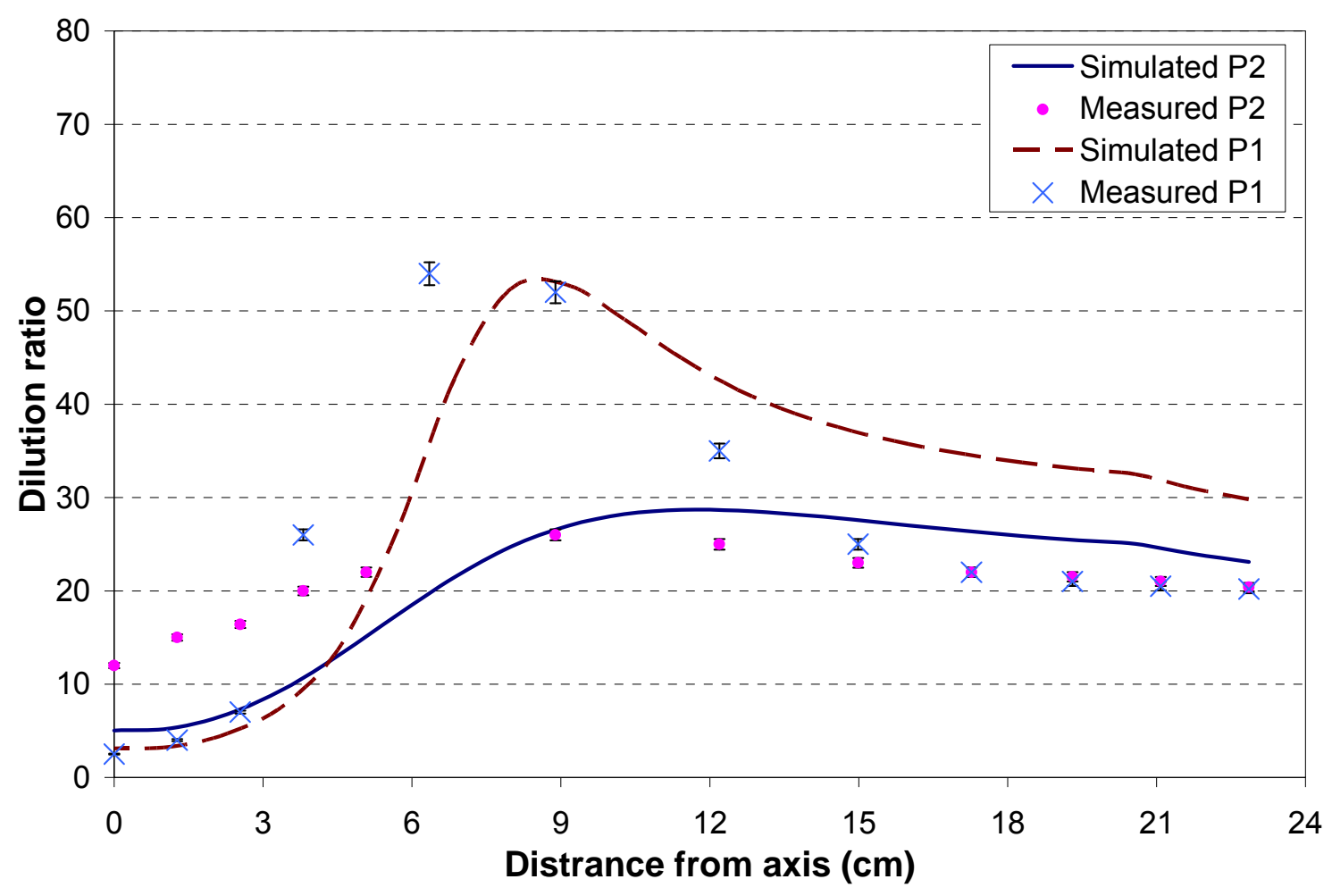

Figure 18 Measured and simulated dilution ratios 
The results in Figure 18 indicate that the CFD model could estimate the dilution ratio profiles across tunnel sections with reasonable capability of prediction. Differences between simulation and measurement results can be explained by model simplification, inaccuracy of sample probe pinpointing, and influence on the flow field caused by sample probe intrusion and suction. The purpose of the CFD model was not for accurate prediction of dilution flow behaviors, but for guidance of locating sampling ports and provisions of auxiliary information for particle size distribution measurements. Assumptions were made to simplify the modeling process, including axisymmetric flow behaviors, adiabatic smooth tunnel walls, and uniform inlet velocity profiles. The measured $\mathrm{NO}_{\mathrm{x}}$ concentration levels across $\mathrm{P} 1$ and $\mathrm{P} 2$ sections verified the assumption of axisymmetric flow behaviors. Thus dilution ratios of one half-side of the cross sections were displayed in Figure 18. Measurement results at P3 and P4 showed nearly identical $\mathrm{NO}_{\mathrm{x}}$ concentration levels across and between both sections, which confirmed simulation results.

\subsubsection{PPMD Test Setup}

The test setup for PPMD dilution test is shown as in Figure 19 and Figure 20. The exhaust pipe was connected to the PPMD exhaust pipe adapter downstream of the DPF. The shop air was regulated to $620.5 \mathrm{kPa}$ (90psi) before feeding into the PPMD to provide dilution air. The QCM part of the PPMD was not used so that all diluted sample went out of the sample out port of the PPMD. The DMS500 sample probe was connected to the sample out port of the PPMD to measure the particle size distributions of PPMD diluted flow. A tee connector was used between the DMS500 sample probe and the sample out port of the PPMD with one end open to the atmosphere. The excess of diluted exhaust flow went to the atmosphere and rest of it was drawn into the DMS500 for size distribution measurement. The MPS dilution stage of the PPMD was configured to run at constant dilution ratio mode instead of proportional sampling mode. Particle size distributions of diluted exhaust flow were measured with different setting values of the dilution ratios. The MPS diluters were set to operate in single dilution stage and double dilution stage to examine the dilution effects of the PPMD on the particle size distributions. 


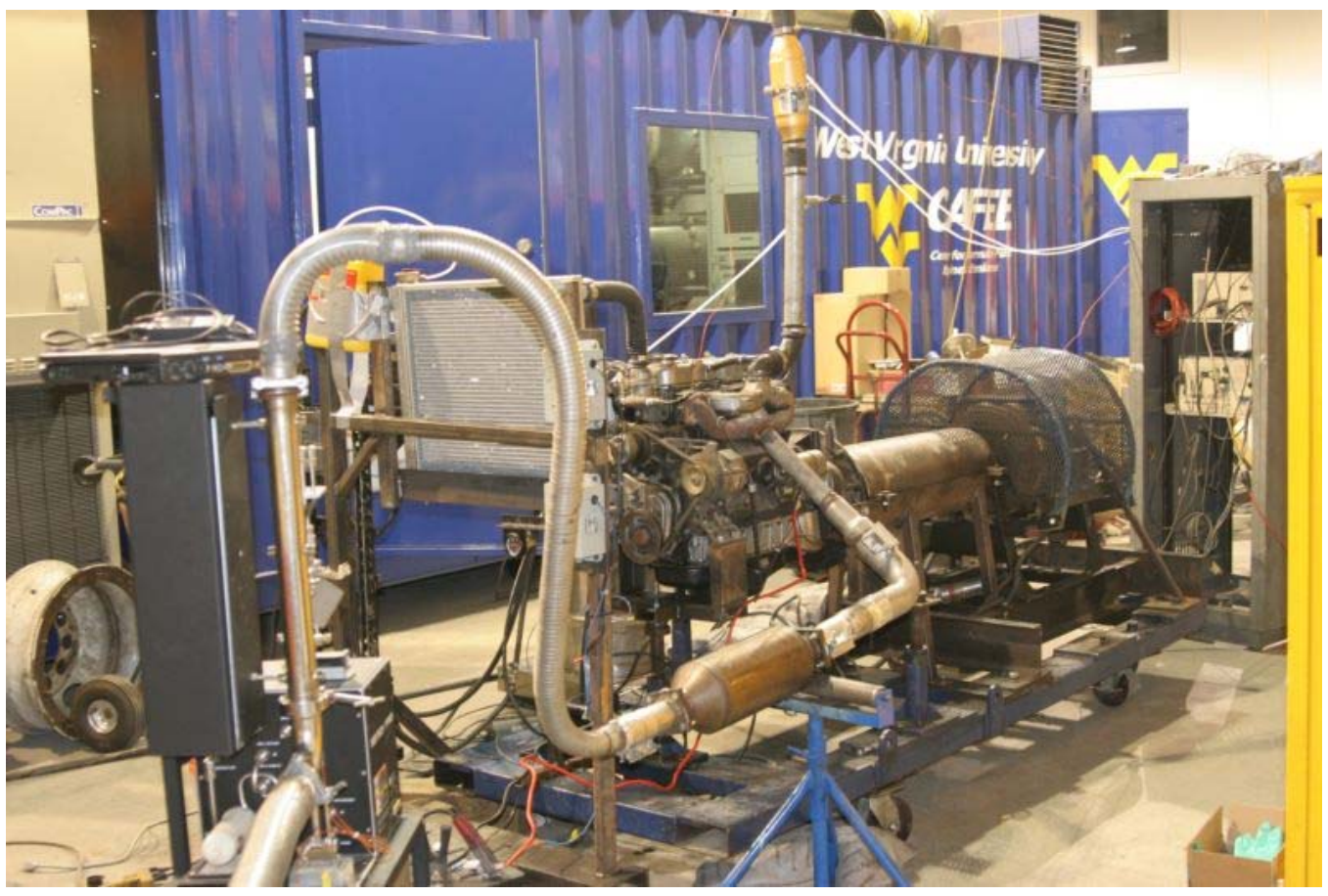

Figure 19 View of PPMD with engine test cell
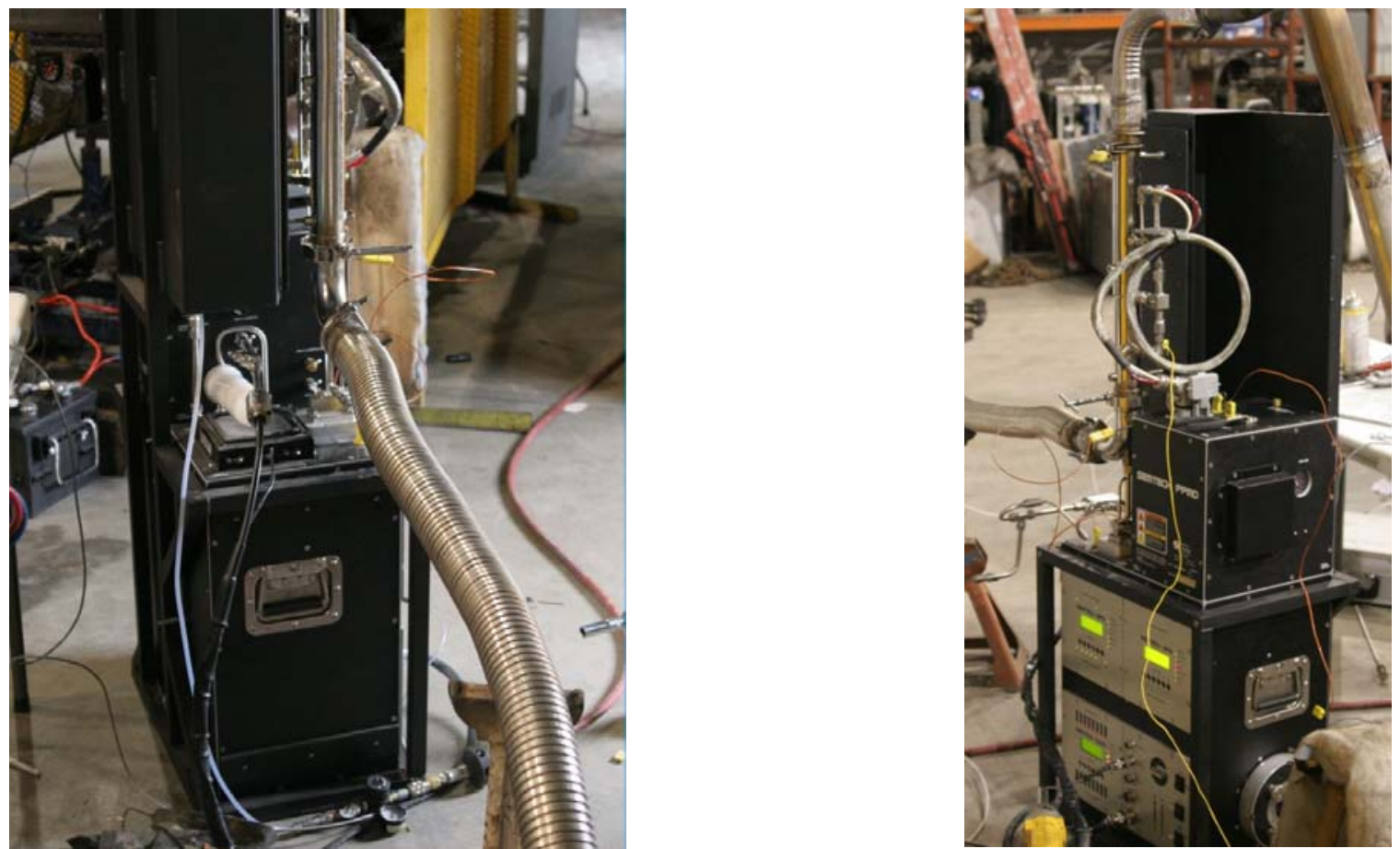

Figure 20 Front and side views of PPMD connections 


\subsubsection{Post-tailpipe Test Setup}

DPM size distributions after the exhaust tailpipe centerline were investigated. Gaseous emissions were also measured to determine the dilution ratios of measured locations. The exhaust pipe was extended from the outlet of the DPF to outside of the buildings where the engine test cell was set up. The outlet of the exhaust tailpipe was supported as horizontally at about 26 inches high. A sample probe with a coned tip was built to take diluted exhaust sample. The sample probe tip was made to reduce sampled area of sampling locations. At the end of the sample probe, the diluted exhaust sample flow was routed to two separate sample lines. One stream of sample flow went to the DMS500 for the measurement of particle size distributions, and other stream of sample flow went to MEXA-7200 gas analyzers for the measurement of gaseous emissions. Figure 21 shows the test site configuration with a magnified view of the sample probe tip. The vehicle shown in the picture was moved away during the test.

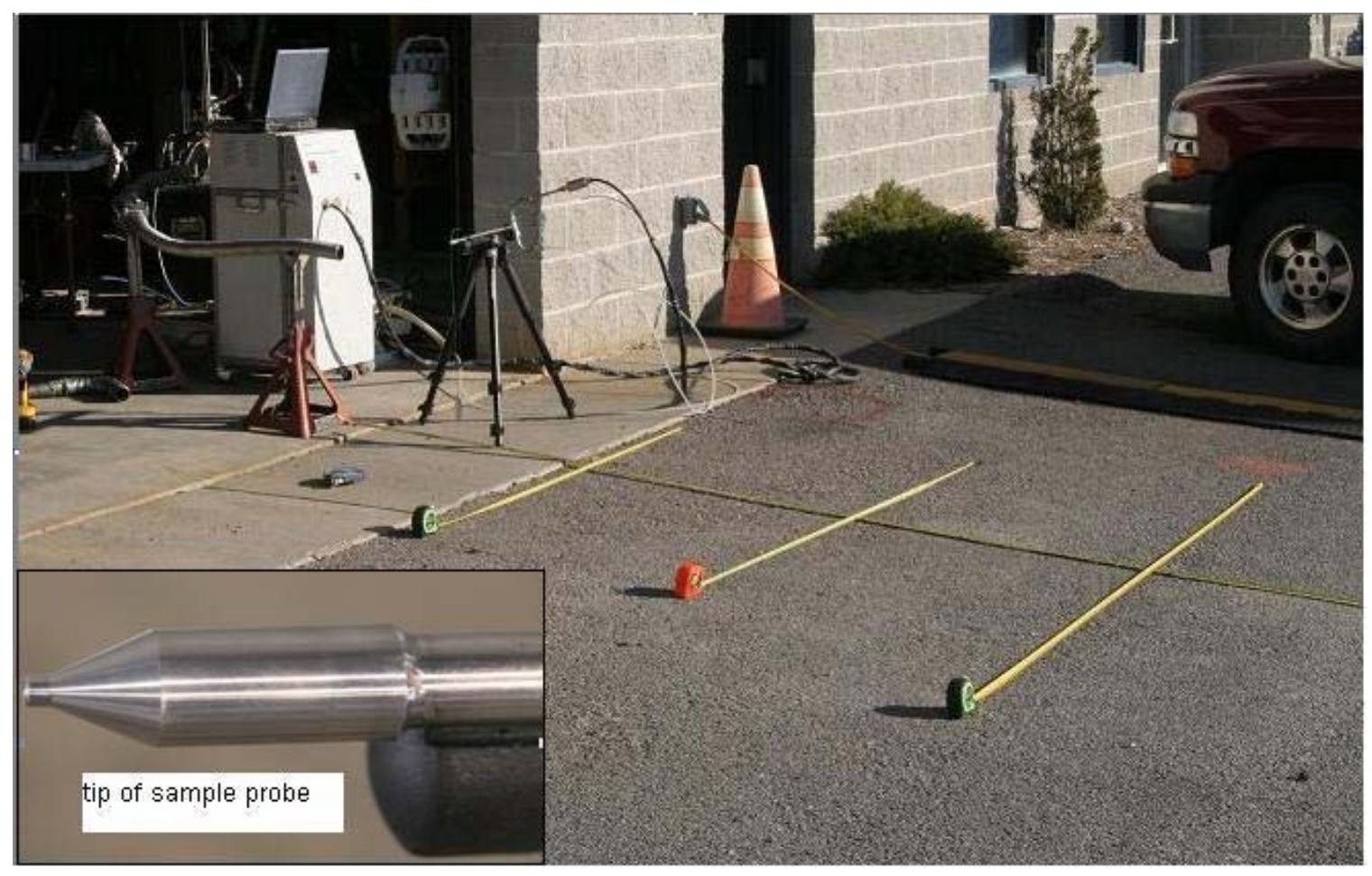

Figure 21 After exhaust tailpipe test setup with view of the sample probe tip 
The sampling locations of the post-tailpipe test were determined during the test. The principle of sampling location selection was to observe and capture changes of dilution ratios and particle size distributions along the post-tailpipe centerline without sharp measurement gradients. Since the ambient post-tailpipe test was conducted in the outdoor environment, a least windy (least windy here means that wind speed is less than $2 \mathrm{mph}$ ) day with high visibility, and no precipitation was selected to perform the test $(01 / 03 / 2009)$ in order to reduce the effects of meteorological conditions. Table 3 shows the historical weather data recorded by the weather station closest to the test site in Morgantown, WV on 01/03/2009. This table shows how the meteorological conditions changed during the test period at a nearby site. However, the real meteorological parameters could be slightly different from the nearby weather station especially for the wind speeds and directions. The whole test was performed between 10:00 AM and 5:30 PM and mainly between 3:00 pm and 4:00 pm. A Kestrel® 4000 Pocket Wind Meter was also used to provide wind speed information during the test. It indicated that the wind was blowing from eastern side of the building (Monongahela River) around $1 \mathrm{mph}$ most of time during the test in the test field. A satellite view of the test site is shown in Figure 22 to provide visual information of the test location and wind.

Table 3 Historical weather station data on 01/03/2009, South Park, Morgantown, WV

\begin{tabular}{|c|c|c|c|c|c|c|c|}
\hline Time (EST): & Temp.: & $\begin{array}{c}\text { Dew } \\
\text { Point: }\end{array}$ & Humidity: & $\begin{array}{c}\text { Sea Level } \\
\text { Pressure: }\end{array}$ & Visibility: & $\begin{array}{c}\text { Wind } \\
\text { Dir: }\end{array}$ & $\begin{array}{c}\text { Wind } \\
\text { Speed: }\end{array}$ \\
\hline $9: 53 \mathrm{AM}$ & $30.0^{\circ} \mathrm{F}$ & $19.0^{\circ} \mathrm{F}$ & $64 \%$ & 30.17 in & 10.0 miles & Calm & Calm \\
\hline $10: 53 \mathrm{AM}$ & $33.1^{\circ} \mathrm{F}$ & $19.0^{\circ} \mathrm{F}$ & $56 \%$ & 30.17 in & 10.0 miles & Calm & Calm \\
\hline $11: 53 \mathrm{AM}$ & $35.1^{\circ} \mathrm{F}$ & $19.0^{\circ} \mathrm{F}$ & $52 \%$ & 30.16 in & 10.0 miles & Calm & Calm \\
\hline $12: 53 \mathrm{PM}$ & $37.0^{\circ} \mathrm{F}$ & $15.1^{\circ} \mathrm{F}$ & $41 \%$ & 30.14 in & 10.0 miles & Variable & $3.5 \mathrm{mph}$ \\
\hline $1: 53 \mathrm{PM}$ & $39.9^{\circ} \mathrm{F}$ & $10.9^{\circ} \mathrm{F}$ & $31 \%$ & 30.12 in & 10.0 miles & NNW & $4.6 \mathrm{mph}$ \\
\hline $2: 53 \mathrm{PM}$ & $41.0^{\circ} \mathrm{F}$ & $12.0^{\circ} \mathrm{F}$ & $31 \%$ & 30.13 in & 10.0 miles & NNE & $4.6 \mathrm{mph}$ \\
\hline $3: 53 \mathrm{PM}$ & $39.0^{\circ} \mathrm{F}$ & $12.9^{\circ} \mathrm{F}$ & $34 \%$ & 30.14 in & 10.0 miles & North & $4.6 \mathrm{mph}$ \\
\hline $4: 53 \mathrm{PM}$ & $37.0^{\circ} \mathrm{F}$ & $12.9^{\circ} \mathrm{F}$ & $37 \%$ & 30.16 in & 10.0 miles & Calm & Calm \\
\hline $5: 53 \mathrm{PM}$ & $34.0^{\circ} \mathrm{F}$ & $12.9^{\circ} \mathrm{F}$ & $42 \%$ & 30.17 in & 10.0 miles & NNE & $3.5 \mathrm{mph}$ \\
\hline
\end{tabular}




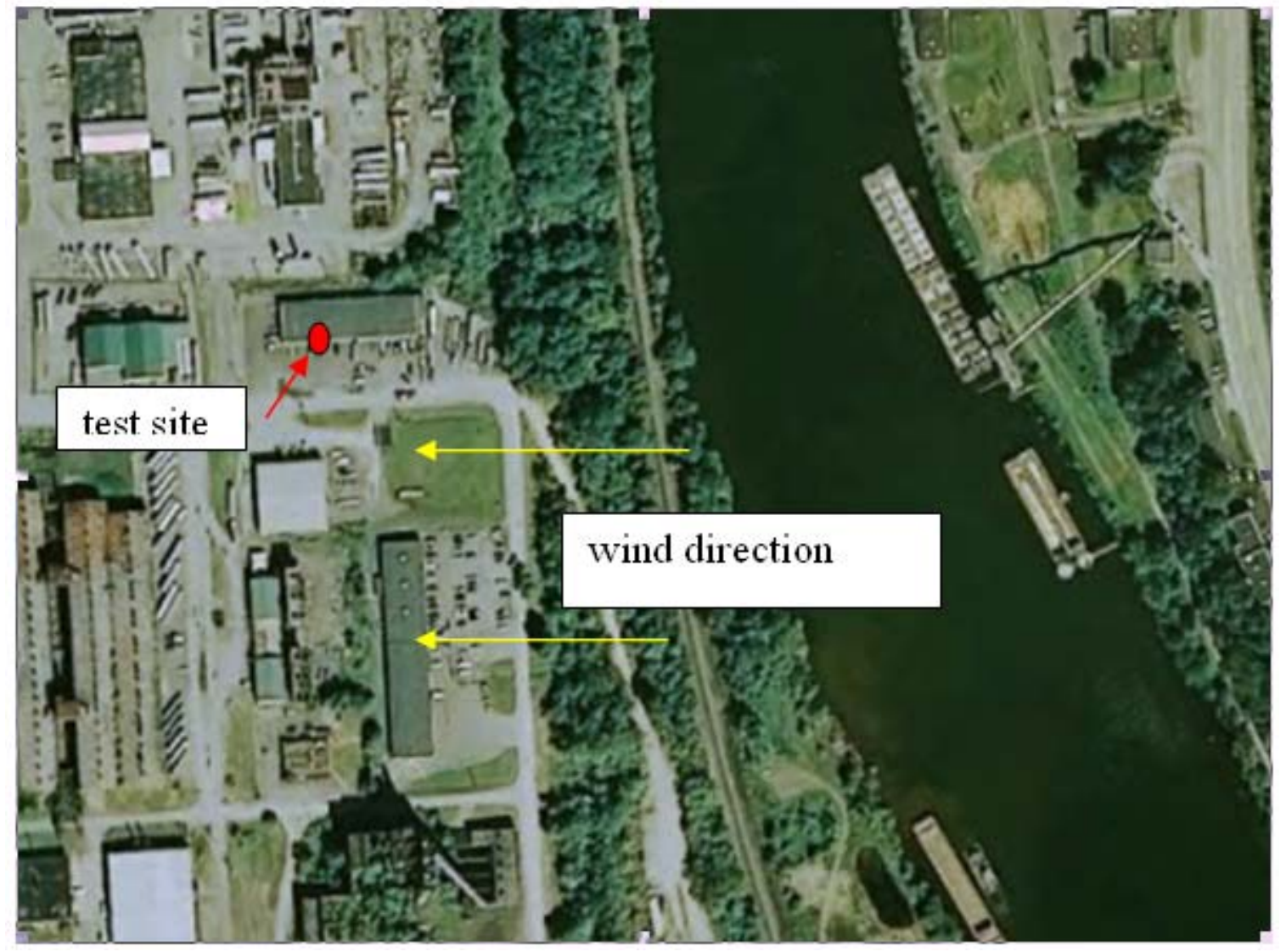

Figure 22 Satellite view of test site and illustration of wind 


\section{TRANSPORTABLE LOW EMISSIONS MEASUREMENT LABORATORY}

This chapter provides an overview of the design and construction techniques used in the new transportable laboratory. A major part of this chapter is reorganized verbatim copy of publications $[140,141$,$] . The various components making up the new$ transportable emissions testing laboratory were being verified at the time of this dissertation, and further results will be reported. In addition, in order to conform to the CFR Part 1065 standard or performance for this new transportable laboratory, it was also important to have engine fuels (40 CFR §1065.704), lubricants (40 CFR §1065.740), and engine work input and output (40 CFR $§ 1065.210$ ) to meet 1065 standards.

The completion of this transportable emissions testing laboratory was the results of the whole CAFFE's teamwork. The author conducted the PM sampling system's parametric and CFD modeling study and was involved in the day to day design and manufacturing processes.

\subsection{Introduction}

In response to the growing concerns of environmental pollution, the US EPA adopted more stringent new emissions policies. For example, certification standards for on highway HDDEs were reduced from $0.1 \mathrm{~g} / \mathrm{bhp}-\mathrm{hr}$ to $0.01 \mathrm{~g} / \mathrm{bhp}-\mathrm{hr}$, for PM, and $2.5 \mathrm{~g} / \mathrm{bhp}-\mathrm{hr}$ to $0.20 \mathrm{~g} / \mathrm{bhp}-\mathrm{hr}$, for $\mathrm{NO}_{\mathrm{x}}$, in 2007 , representing an order of magnitude reduction in pollutant levels. In the meantime, pressures on fossil fuel supply have increased the demand for alternative fuels and the Department of Energy (DOE) has made stricter energy policies to meet the need today and plan for the future. Although the US EPA has advanced emissions certification standards substantially over past decades, the primary measurement techniques for heavy duty engine emissions remained largely unchanged $[38,34]$. A schematic of the emissions sampling system of WVU's new low emissions mobile laboratory is included in Figure 23. The biggest change in schematic shown in Figure 23 compared to what shown in Figure 2 is the inclusion of gaseous sampling ports and analyzer blocks. 


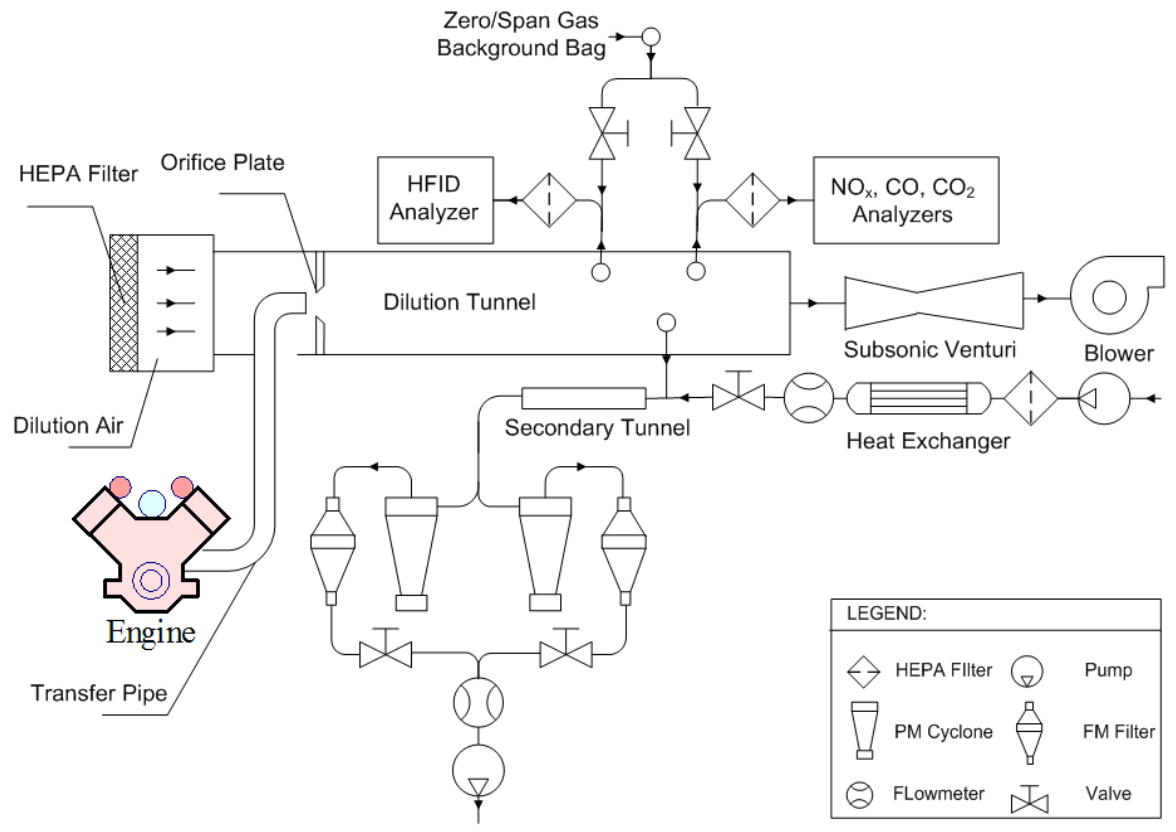

Figure 23 Schematic of emissions sampling system

The emissions sampling system shown in Figure 23 is based on the SSV-CVS concept. A primary dilution tunnel is used to dilute the full flow of engine exhaust with HEPA-filtered air, the mixture being metered by a subsonic venturi and a speedcontrolled blower. At the primary tunnel's entrance, the entire engine exhaust is introduced, via a transfer pipe, into the primary tunnel plenum. The exhaust is mixed with HEPA filtered dilution air immediately upstream of a mixing orifice plate. The two streams are further mixed to a CFR Part 1065-compliant minimum dilution ratio of 2, in the mixing region downstream from the orifice plate. At a downstream distance of approximately 10 times the tunnel diameter, sample probes are installed to collect diluted gaseous exhaust samples, as well as aerosol samples for analysis of PM. The gaseous samples are then delivered to a Horiba MEXA-7200D gaseous emissions analyzer in order to quantify concentrations of $\mathrm{THC}, \mathrm{CO}, \mathrm{CO}_{2}$, and $\mathrm{NO}_{\mathrm{x}}$. THC emissions are measured with a Heated Flame Ionization Detector (HFID), $\mathrm{CO}$ and $\mathrm{CO}_{2}$ emissions are measured with Non-Dispersive Infrared (NDIR) gas analyzers, and $\mathrm{NO}_{\mathrm{x}}$ emissions are measured with both heated (wet) and cold (dry) chemiluminscence analyzers. The PM sample is drawn into a secondary dilution tunnel, further diluted with conditioned HEPA filtered air, and passed through a cyclone separator in order to remove mechanically 
generated coarse particles. The flow is then drawn across a PM sample filter for gravimetric analysis.

Using a partial flow tunnel as an alternative to a full flow CVS has been investigated for several years [37, 142 - 145]. The partial dilution tunnel is portable, and inexpensive to use compared to the full flow CVS system. However, the partial dilution system is more complex and more difficult to calibrate. The US EPA did not approve the use of partial dilution tunnels for HDDE PM emissions tests in CFR Part 1065. The US EPA determined there was insufficient evidence to support that a partial dilution tunnel correlated well enough to a full scale tunnel in measuring diesel emissions. However, using a partial flow dilution tunnel for HDDE emissions test is allowed upon the approval of the US EPA.

\subsection{Laboratory Overview}

The housing for the new transportable laboratory is a reconstructed $9.1 \mathrm{~m}$ (thirtyfoot) long cargo container which houses a HEPA primary dilution unit, two primary fullflow dilution tunnels, a subsonic venturi, a secondary particulate matter sampling system, a gaseous emissions analytical bench instrumentation system, a computer-based DAQ and control system, a heating, ventilating and air conditioning (HVAC) system, and chassis dynamometer control systems. In addition to the container view shown in Figure 14, Figure 24 presents another photograph of the outside view of the container.

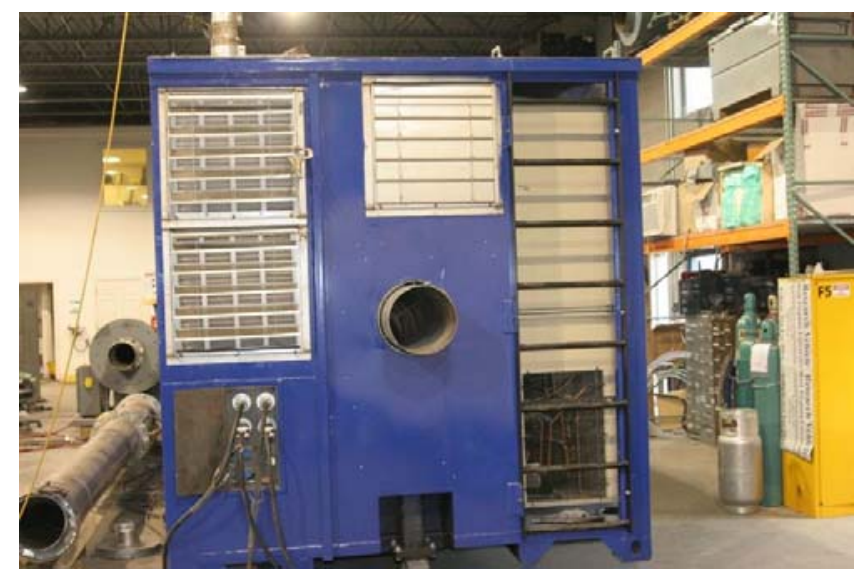

Figure 24 View of transportable laboratory container 
The two primary dilution tunnels inside the container, of $0.46 \mathrm{~m}$ (18 inches) ID and $6.1 \mathrm{~m}$ (20 feet) long, were designed to provide dedicated measurement capability for both low PM emissions ("clean") vehicles (with the upper tunnel referred as the "clean tunnel"), as well as traditional diesel-fueled vehicles with high PM levels (lower tunnel referred as "dirty tunnel"). This provision reduces tunnel history effects between test programs of differing exhaust emission composition. Figure 25 shows the simplified 3-D schematic of the housing cargo container.

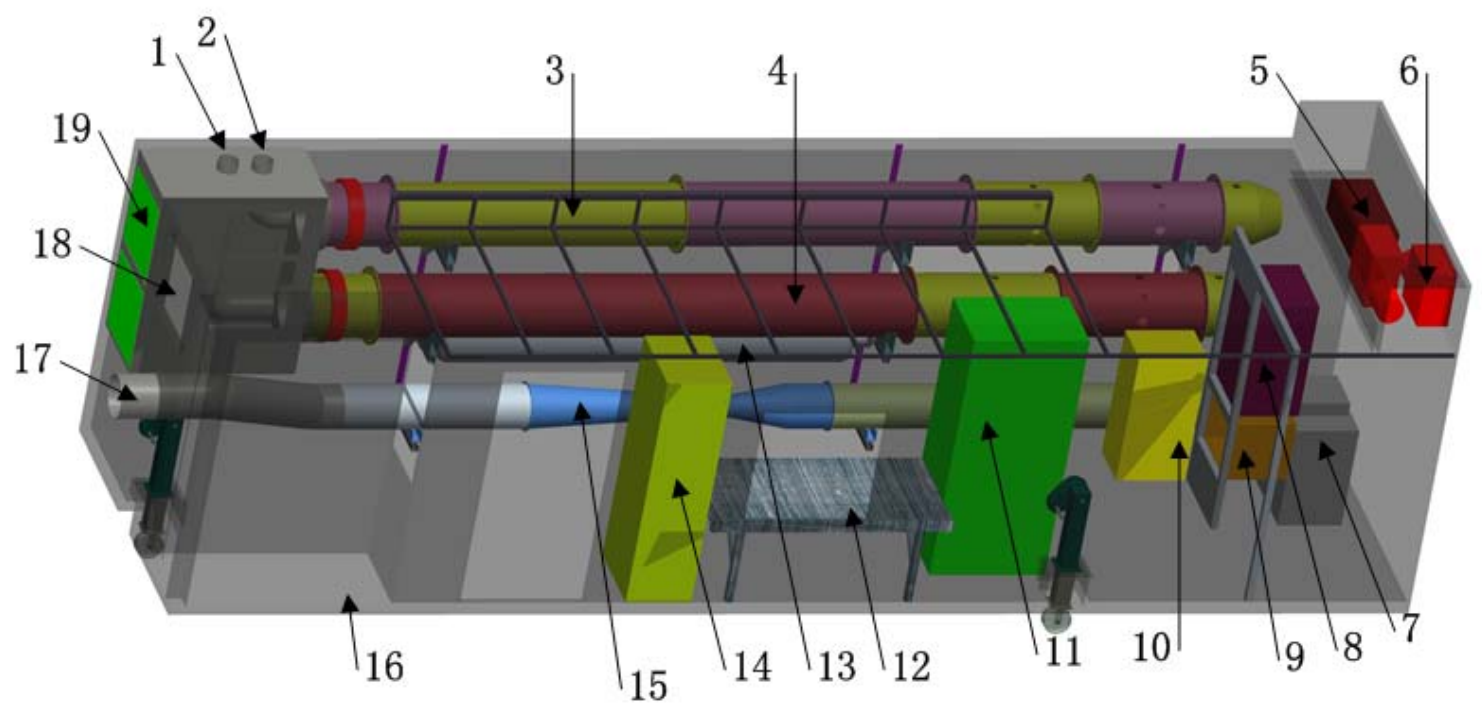

Figure 25 3-D Schematic of laboratory container

1- Exhaust inlet of dirty tunnel; 2- Exhaust inlet of clean tunnel; 3-Clean tunnel; 4- Dirty tunnel; 5- Air compressor; 6- Vacuum pumps; 7- Oven; 8- PM sampling box; 9Glove box; 10- Zero air generator; 11- MEXA-7200D motor exhaust gas analyzer; 12Computer table; 13- Air tank; 14- DAQ rack; 15- Subsonic venturi; 16- Air conditioner deck; 17- Outlet to blower; 18-Ventilation fan; 19- HEPA filters

As shown in Figure 25, the design of the transportable laboratory conforms to the conceptual diagram shown in Figure 23. A stainless steel plenum box houses two HEPA filters for filtering primary dilution air, as well as twin dual-wall exhaust transfer inlet tubes dedicated as exhaust inlets for the upper and lower tunnels. The HEPA plenum is connected into the main dilution tunnels, which are selectively connected to the subsonic 
venturi via stainless elbow sections. The air compressor and two vacuum pumps are installed inside a noise isolating overhead. An air tank stores compressed air and provides shop air to the zero air generator (a device removes PM and THC) for instrumentation use. A PM sampling box for the secondary dilution tunnels is located alongside the primary tunnels, downstream of tunnels' sample zones. The secondary PM dilution tunnel of either the dirty or clean tunnel is connected to the PM sampling box for PM measurement during the test. Most key components of the transportable laboratory have been shown in Figure 25. However, views of some components, such as secondary tunnels, secondary dilution air conditioning unit, and gas bottle rack are omitted for clarity.

The laboratory grade analytical system conforms to the applicable specifications defined in CFR Part 1065. It can be transported to virtually any location where emissions testing is to be conducted, with or without WVU's transportable medium or heavy duty chassis dynamometers. The design of the heavy duty chassis dynamometer and the results of emissions testing using the previous generations of WVU's mobile testing laboratories have been discussed in prior publications [146, 147]. The system may even be loaded onto a flatbed trailer to conduct emissions tests while the tractor vehicle is operated over the road. Currently, a Landoll 435 trailer bed is used for this housing container at WVU.

\subsection{Parametric study using Simulink ${ }^{\circledR}$}

For diesel engines to meet the 2007 standard, they must practically be equipped with catalyzed particulate filters. The PM downstream of the filter composition is expected to be mainly composed of both volatile and semi-volatile materials [35]. The current sampling protocol creates technical challenges for the accurate measurement of volatile PM mass. The main challenge is to capture and measure a very low PM mass deposited on a filter, but other factors including filter handling, filter media employed, artifact, sampling, system conditioning, and accurate monitoring of dilution parameters could also affect measurement results $[35,45]$. 
To account for the 2007 PM measurement challenges, the EPA defined PM collection specified in 40 CFR Part 86 and in CFR Part 1065. The filter face temperature requirement was changed from "below $52^{\circ} \mathrm{C}$ " to a range of "between $42^{\circ} \mathrm{C}$ and $52^{\circ} \mathrm{C}$ $\left(47^{\circ} \mathrm{C} \pm 5^{\circ} \mathrm{C}\right) "$ [2]. The EPA introduced a subsonic venturi as a third alternative device for the CVS in 40 CFR, Part 86 and in Part 1065 as shown in Figure 1. In order to reduce the effects of humidity and stray particle capture further during filter weighting, the EPA required more stringent specifications on ambient conditions for filter stabilization and weighting (a temperature at $22^{\circ} \mathrm{C} \pm 3^{\circ} \mathrm{C}$ and a dew point of $9.5^{\circ} \mathrm{C} \pm 1{ }^{\circ} \mathrm{C}$ for the filter stabilization environment and a temperature at $22^{\circ} \mathrm{C} \pm 1{ }^{\circ} \mathrm{C}$ and a dew point of $9.5^{\circ} \mathrm{C} \pm$ $1{ }^{\circ} \mathrm{C}$ for the immediate microbalance workstation environment) [38]. The EPA also recommended a Class 1000 clean room (A class 1000 clean room maintains less than 1000 particles larger than 0.5 microns in each cubic foot of air space) as the microbalance and filter stabilization environment [38]. With changes in both the regulation of the secondary tunnel as well as the filter media, filter handling, and weighting chamber, the 2007 regulations attempted to improve quantification, while reducing the variation of measured PM mass emissions [35].

However, the EPA left some room for interpretation in the 2007 regulations. For example, the EPA does not give specifications for the secondary tunnel's volume and length. It does not state any restrictions on the primary and secondary dilution ratios. There is only an upper bound of $100 \mathrm{~cm} / \mathrm{sec}$ for the filter face velocity. It does not limit the filter media to one specific material. It does not provide an upper limit for the primary and secondary dilution air temperature. The effects of these factors on PM measurement have been recognized but are still not fully understood $[35,45]$.

In light of the flexibility in sampling geometry for the 2007 standard PM sampling system, a systematic study of variables affecting PM measurement was performed. The main objective of this parametric study was to quantify the design and operating envelope that will allow for compliance with 2007 standards. 


\subsubsection{System Model}

\section{Overview}

Exhaust is diluted using a CVS system. Gaseous emissions are sampled from the primary dilution tunnel and analyzed by instruments capable of continuous measurement. PM is diluted twice before being collected on a particulate filter. Figure 26 shows the highest-level diagram of a Simulink ${ }^{\circledR}$ model for 2007 PM emission sampling system. "Simulink ${ }^{\circledR}$ is a platform for multi-domain simulation and Model-Based Design for dynamic systems. It provides an interactive graphical environment and a customizable set of block libraries [148]."

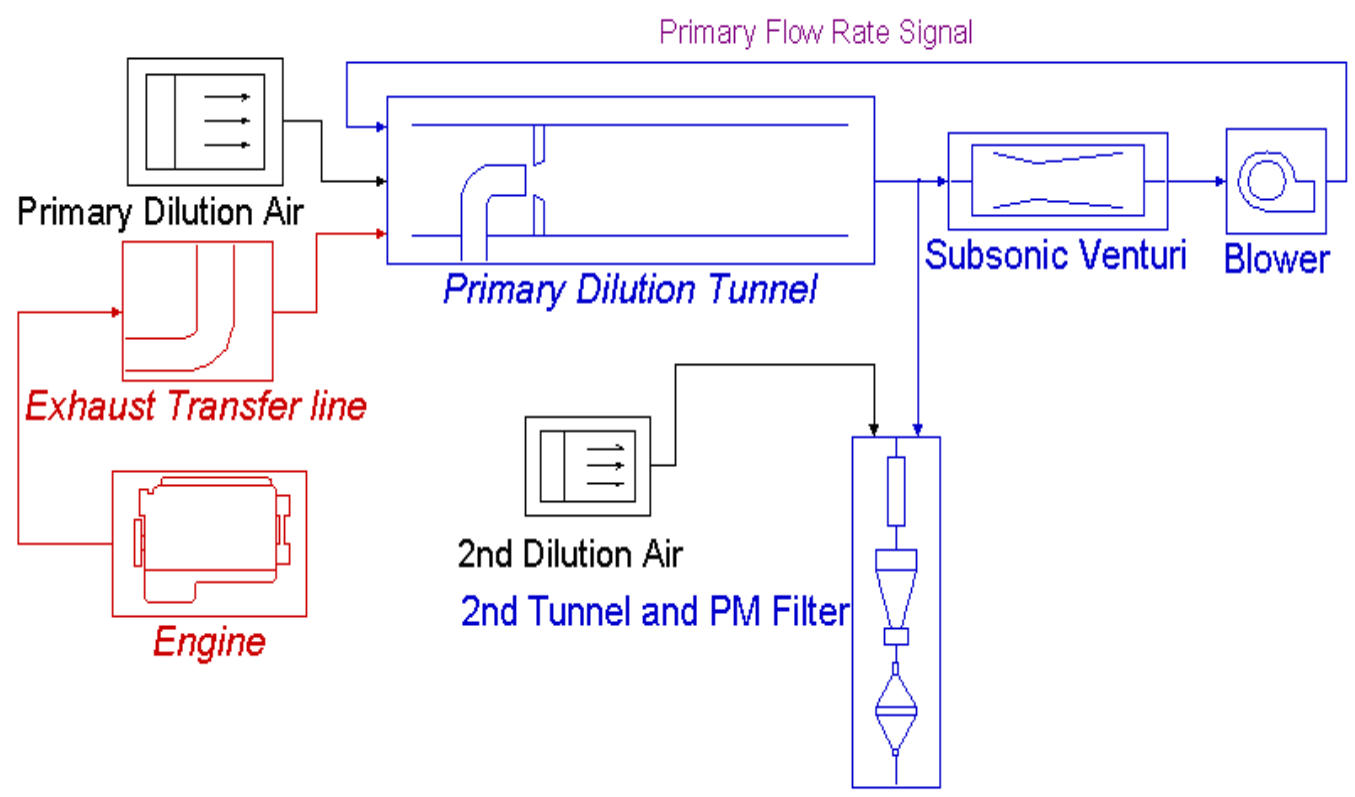

Figure 26 Simulink ${ }^{\circledR}$ model of PM sampling system-highest level

Modeling of the PM sampling system shown in Figure 26 was based upon the physical configuration shown in Figure 23. The SSV-CVS concept was used because it offers both a high degree of control and excellent energy efficiency. This was also the basic design selected for use at WVU for 2007 compliant measurement. Other geometries within the constraint of the 2007 EPA test procedures are available and can also be examined by altering this model. 


\section{Gas Composition}

The exhaust's chemical composition and its thermodynamic properties change during a real time test with corresponding engine conditions, flow temperatures, and dilution ratios.

At idle, it is common to have a diesel engine run at an air fuel (A/F) ratio of 100. At full power, most diesel engines need to run near stoichiometrically with the lowest $\mathrm{A} / \mathrm{F}$ ratio. The stoichiometric ratio for diesel fuel varies slightly depending upon the fuel's composition. A diesel fuel with an average molecular formula $\mathrm{C}_{12.5} \mathrm{H}_{22.2}$ was used in this system model. It has a stoichiometric $\mathrm{A} / \mathrm{F}$ ratio of 14.5 . To simplify modeling of exhaust and to explore a wide $\mathrm{A} / \mathrm{F}$ range, it was assumed that $\mathrm{A} / \mathrm{F}$ was 16 (which is an extreme case that would imply heavy smoking) when the engine runs at full power and 100 when the engine runs at idle.

Considering that small pressure variation has little effect on the exhaust's thermodynamic properties, exhaust pressure during a test can be considered as a constant value at $0.1 \mathrm{MPa}$, which is $0.32 \%$ lower than atmospheric pressure considering pressure drops in tunnels. The temperature of exhaust gas was the variable needed to calculate exhaust's thermodynamic properties after its composition was determined. Thermodynamic properties of exhaust at $\mathrm{A} / \mathrm{F}$ ratios of 16 and 100 were calculated for mixtures of all gaseous compositions using a third order virial equation of states based on the gas property data published by The National Institute of Standards and Technology. Results are shown in Table A1, Figure 27, Figure 28, and Figure 29.

As shown in Table A1, dry air has nearly identical density to engine exhaust on a wet basis. Their average molecular weights are almost equal. When the diesel engine was running at idle, very little fuel was injected into the combustion chamber to burn. Then, the exhaust at idle was mostly composed of air, and it was comparable to dry air. Any differences between the two should be negligible when the exhaust was further diluted in the primary and the secondary tunnel. It is reasonable to approximate the exhaust at idle as dry air for concerned thermodynamic properties. 
Dry air's kinematic viscosity value is, on average, $8 \%$ higher than the value of raw engine exhaust at full power (Figure 27). Kinematic viscosity was used to determine the flow's condition (laminar, transitional, or turbulent) by calculating the Re. Then the corresponding equations were selected for the system model. For real time tests, exhaust flow through the test system would be a fully turbulent flow, and an $8 \%$ difference in kinematic viscosity value should not change the flow's condition based on the fact that the $\operatorname{Re}$ is maintained at a high value $(>10,000)$ during the emissions test for the primary dilution flow.

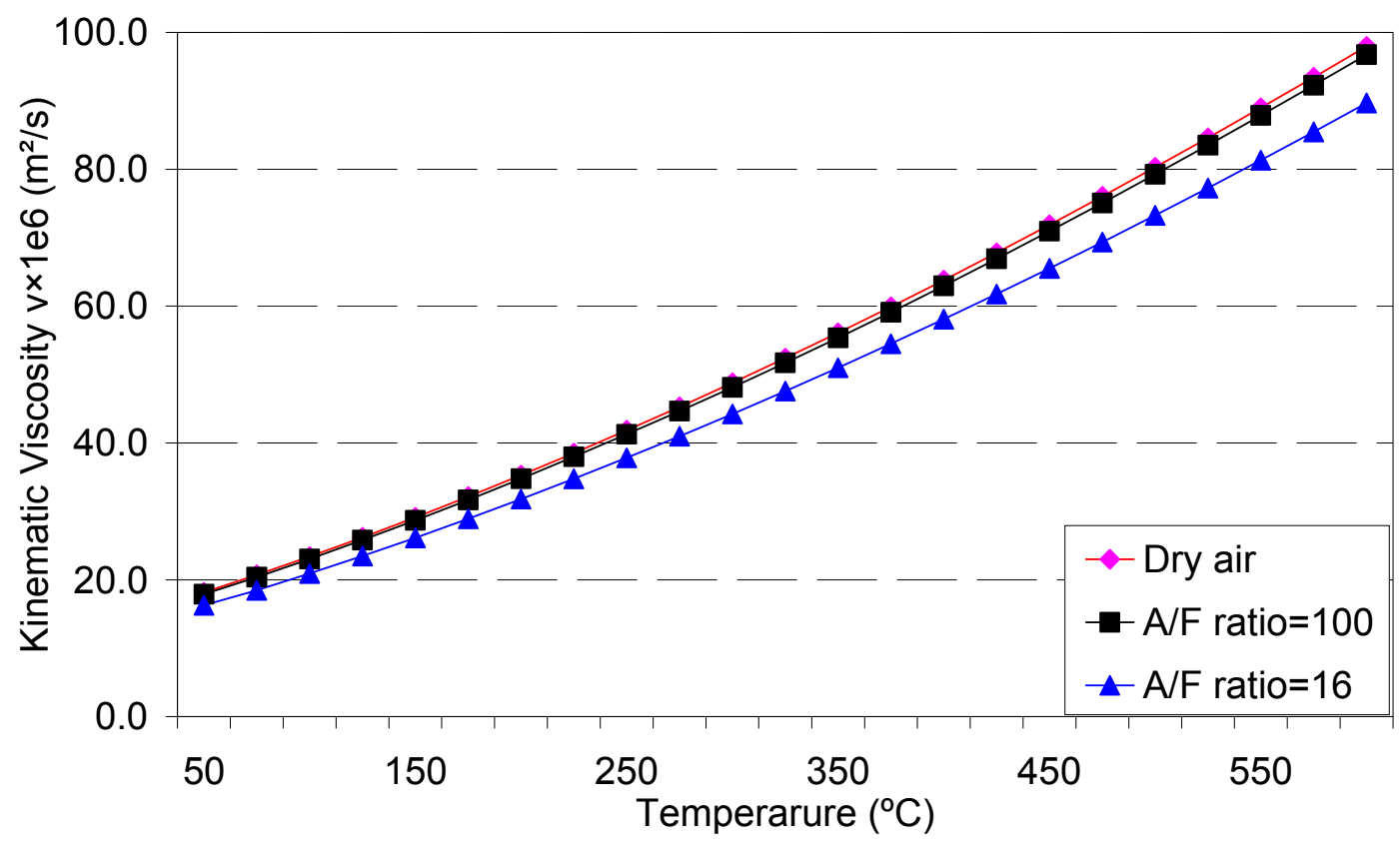

Figure 27 Kinematic viscosity of dry air and exhaust at different air fuel ratios

Dry air has an average of 15\% lower specific heat value and $8.5 \%$ higher thermal conductivity value than those of raw engine exhaust at full power (Figure 28, Figure 29). When the engine is running at full power, the typical dilution ratio inside the primary tunnel is 3 to 5 (CFR Part 1065 require a minimum value of 2). As a result, simply using dry air's properties to model the exhaust at full power through the test system is unsatisfactory. 


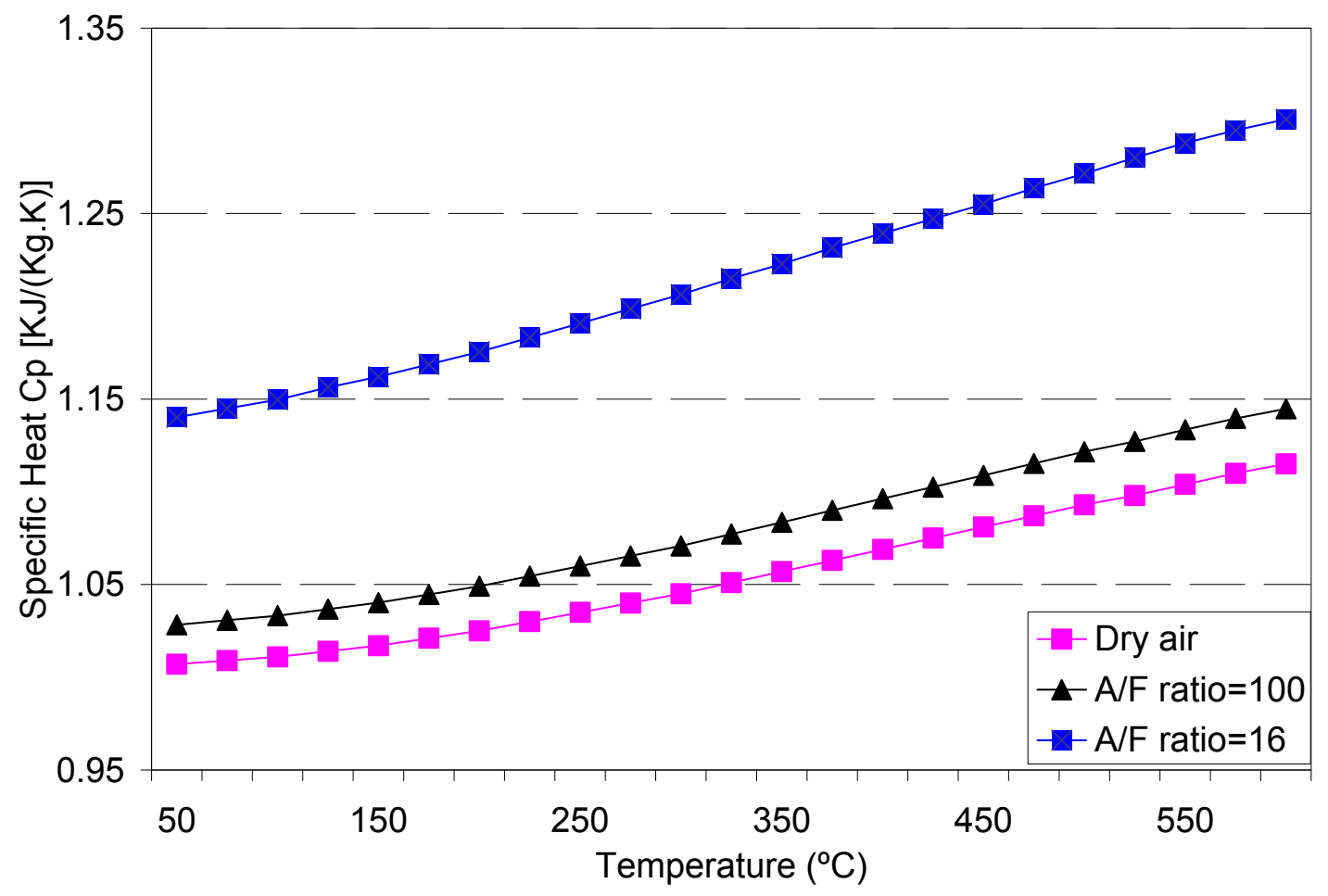

Figure 28 Specific heat of dry air and exhaust at different air fuel ratios

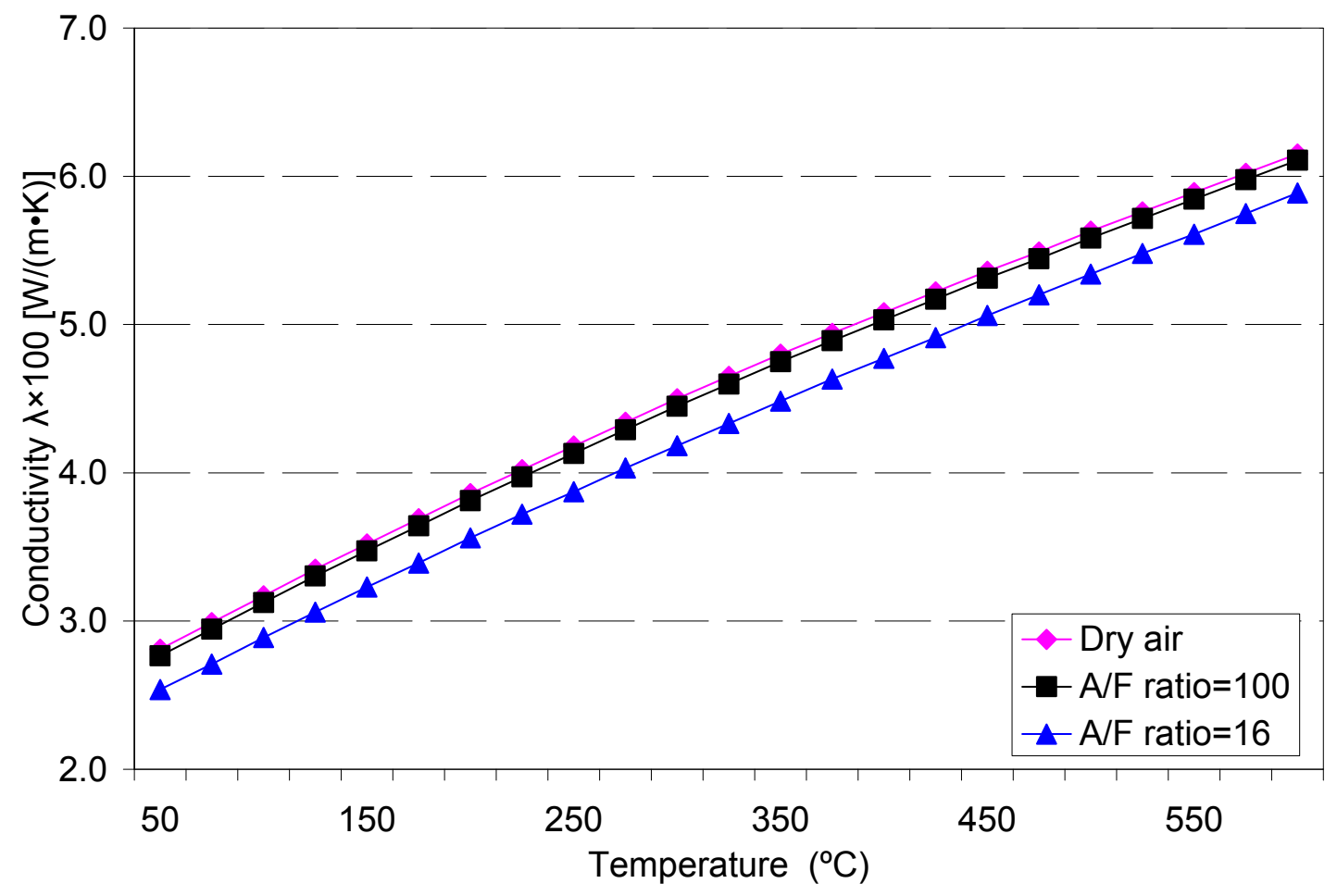

Figure 29 Thermal conductivity of dry air and exhaust at different air fuel ratios 


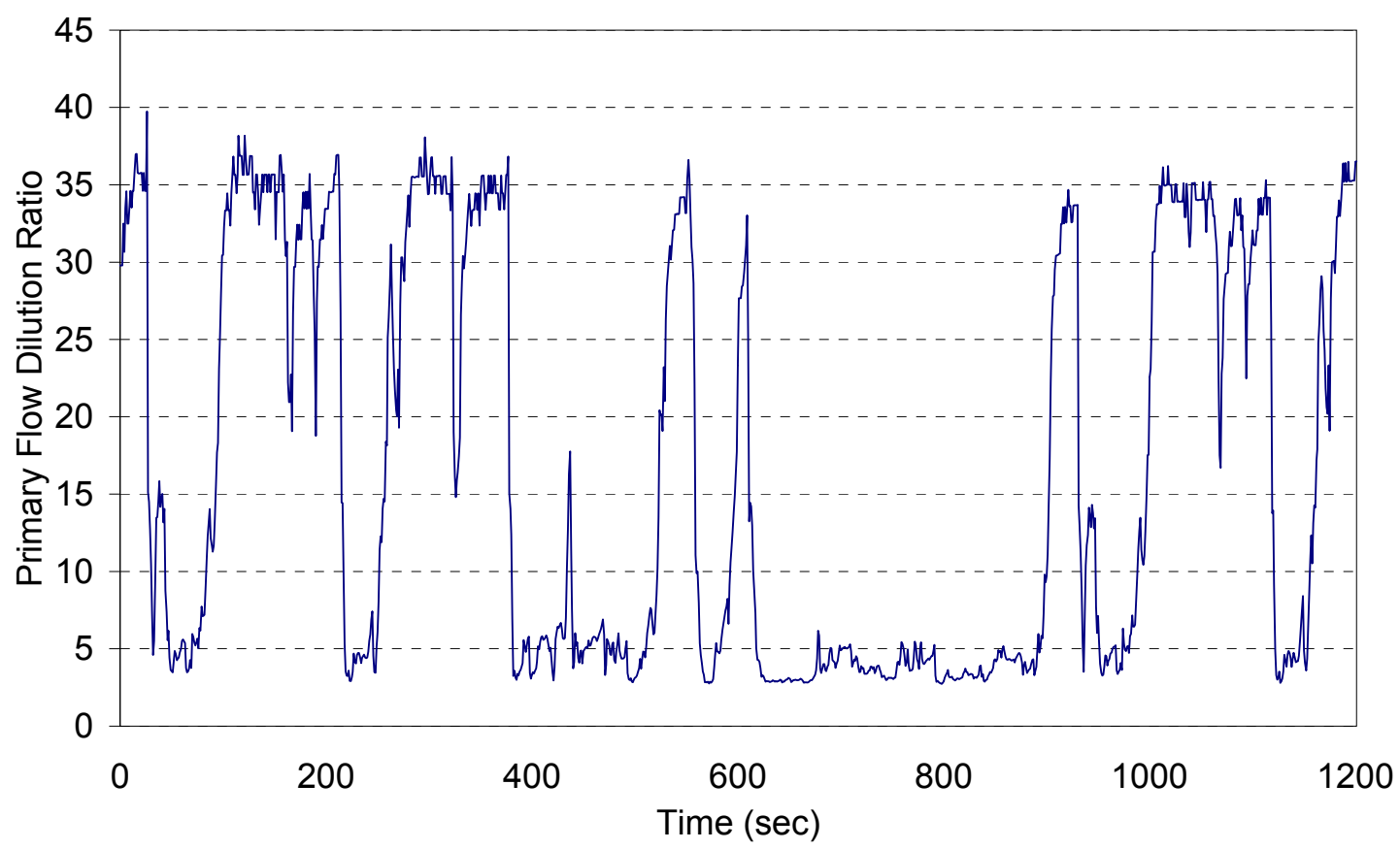

Figure 30 Dilution ratios of the primary tunnel flow for a 2004 Cummins ISM370 engine FTP test

The higher conductivity value of the most diluted exhaust (mostly air) than the raw exhaust at full power flowing through the test system enhances the magnitude of forced convection heat transfer between the flow and the walls, because the Nusselt number $(\mathrm{Nu})$ represents the ratio of the convective heat transfer coefficient to the conductive heat transfer coefficient. Hence, more energy is transfered. Meanwhile, the reduction in the specific heat value of the most diluted exhaust will increase or decrease the flow's temperature faster given same amount of heat exchanged. The differences of the specific heat and conductivity values between the dry air and the raw exhaust at full power can cause a $27 \%$ deviation on the calculated temperature change for the dry airflow compared to the raw engine exhaust flow at full power. Figure 30 shows that when the engine was running at near full power, the dilution ratios were about 3 to 5 when the primary flow was maintained around $1.0 \mathrm{~m}^{3} / \mathrm{sec}$, which is a value typical for an on-road diesel engine test. Calculations showed that at such dilution ratios, around a $5 \%$ to $9 \%$ error in simulated flow temperature changes occurred by using only the dry air's thermodynamic properties. 
Finally, the exhaust's chemical composition and its thermodynamic properties, except density, were modeled as a function of the $\mathrm{A} / \mathrm{F}$ ratio and the dilution ratio. Exhaust's density was modeled as being identical to dry air's density.

\section{$\underline{\text { Relative Humidity }}$}

It is known that the relative humidity of diluted exhaust can affect the total measured PM number concentration. Higher relative humidity usually results in a higher number of PM nano-particles [45]. However, the effect of realative humidty on PM emissions is minimal and not fully quantitatively understood [45]. Since the increased particle number from particle nucleation had negligible mass. Exhaust humidity was not included as a factor affecting PM measurement in this model. PM mass was defined as the mass retained by a filter after standardized conditioning.

\section{Heat Transfer}

Temperature modeling of the diluted exhaust gas through the secondary tunnel and PM filter surface is critical for the compliance of the PM sampling system with the 2007 regulations. The primary tunnel was modeled as a round pipe with its outside surface exposed to ambient temperature. The secondary tunnel was modeled as a round pipe with an isothermal wall. To simplify the modeling of heat transfer, it was also assumed that the exhaust and dilution air were mixed immediately after the exhaust was injected into the primary tunnel. In reality, dilution air may provide an annular sheath for a short distance.

The Re number associated with the flow was first calculated to determine the flow's condition (laminar, transitional, or turbulent). Then, the length of thermal developing region was identified. Finally, the entrance effects were considered for choosing corresponding equations within the model to calculate $\mathrm{Nu}$ and convective heat transfer coefficients. Although there are some analytical solutions for heat transfer coefficients of laminar flows available, most equations used for transitional and turbulent flows are based on empirical data $[151,152]$. Applications of those empirical equations usually have restriction on the value range of Prandtl number (Pr) of the gas flowing 
through the tunnel $[151,152]$. With an exhaust gas Pr of approximately 0.7 , empirical relationships that considered the entrance effects for calculating $\mathrm{Nu}$ were used in this Simulink ${ }^{\circledR}$ model [152].

For a laminar flow through a round tube, the average value of $\mathrm{Nu}$ for the thermal developing region is 3.66 if the tube has an isothermal wall and 4.36 if it has a uniform wall heat flux [152]. When the laminar flow was entirely in thermal entrance region, $\mathrm{Nu}$ was determined by the equation of Kays and Perkins [152, 153]:

$$
N u=3.66+\frac{0.688 \operatorname{Re} \operatorname{Pr}(d / X)}{1+0.04\left[(\operatorname{Re} \operatorname{Pr}(d / X)]^{2 / 3}\right.}
$$

where

$\mathrm{d}=$ inner diameter of the tube $(\mathrm{m})$

$\mathrm{X}=$ length of tube $(\mathrm{m})$.

If a flow were at the transitional condition from laminar to turbulent ( $\mathrm{Re}$ between 2,300 and 10,000), $\mathrm{Nu}$ would be obtained by Gnielinski's empirical expression [152, 154]:

$$
N u=0.0214\left(\operatorname{Re}^{0.8}-100\right) \operatorname{Pr}^{0.4}\left[1+\left(\frac{d}{X}\right)^{2 / 3}\right]\left(\frac{T_{f}}{T_{w}}\right)^{0.45}
$$

where

$$
\begin{aligned}
& T_{f}=\text { mean flow temperature }(\mathrm{K}) \\
& T_{w}=\text { wall temperature }(\mathrm{K}) .
\end{aligned}
$$

When a flow inside the tunnel becomes fully turbulent, with Re being greater than 10,000 , the system model calculates Nu using B. S. Peukhov's relationship $[152,155]$ :

$$
\begin{gathered}
N u=\frac{\frac{f}{8} \operatorname{Re} \operatorname{Pr}}{1.07+12.7\left(\frac{f}{8}\right)^{1 / 2}\left(\operatorname{Pr}^{2 / 3}-1\right)} \\
f=(0.790 \ln (\mathrm{Re})-1.64)^{-2}
\end{gathered}
$$

Equation 4. 3

Equation 4. 4 


$$
0.5 \leq \operatorname{Pr} \leq 2000,10^{4} \leq \operatorname{Re} \leq 5 \times 10^{6}
$$

where

$$
f=\text { Darcy friction factor. }
$$

The primary tunnel and its associated flow are required to be configured so that fully turbulent conditions are generated. However, the capability of handling laminar flow inside the primary tunnel gives this model more flexibility and may allow it to be applied to small diameter partial dilution systems in the future.

Heat transfer through convection and radiation occurs outside of the tunnel walls. It was assumed that tunnel was installed horizontally. Churchill and Chu's equations were then used to determine the $\mathrm{Nu}$ of natural convection from the outside surface. Those empirical equations usually had $10 \%$ to $15 \%$ uncertainty levels $[152,156]$ :

$$
\begin{gathered}
N u=\left\{0.60+\frac{0.387(R a)^{1 / 6}}{\left[1+(0.559 / P r)^{9 / 16}\right]^{8 / 27}}\right\}^{2} \\
R a=G r \operatorname{Pr}, \quad G r=\frac{g\left(t_{w}-t_{f}\right) L^{3}}{v^{2}\left(t_{w}+t_{f}\right)}
\end{gathered}
$$

where

$$
\begin{aligned}
& \mathrm{Ra}=\text { Rayleigh number } \\
& \mathrm{Gr}=\text { Grashof number } \\
& \mathrm{g}=9.8 \mathrm{~m} / \mathrm{s}^{2} \\
& t_{w}=\text { solid surface temperature }(\mathrm{K}) \\
& t_{f}=\text { atmospheric fluid temperature }(\mathrm{K}) \\
& \mathrm{L}=\text { characteristic length }(\mathrm{m}) \\
& v=\text { kinematic viscosity }\left(\mathrm{m}^{2} / \mathrm{s}\right) .
\end{aligned}
$$

The above equations apply to a wide range of $\operatorname{Ra}\left(R a \leq 10^{12}\right)$ and are suitable for boundary conditions of constant wall temperature and constant heat flux [152]. 


\section{Exhaust Energy}

When the engine exhaust is drawn into the PM measurement system, energy is also delivered into the system due to high temperature of the exhaust. The cumulative exhaust energy delivered by the engine is defined in the following equation:

$$
E=\int_{0}^{t} c_{p} \dot{m}\left(T_{e}-T_{a}\right) d t
$$

Equation 4. 7

where

$$
\begin{aligned}
& E=\text { cumulative exhaust energy } \\
& \dot{m}=\text { mass flow rate }(\mathrm{kg} / \mathrm{sec}) \\
& c_{p}=\text { heat capacity of exhaust }(\mathrm{kJ} /(\mathrm{Kg} \cdot \mathrm{K})) \\
& T_{e}=\text { exhaust temperature }(\mathrm{K}) \\
& T_{a}=\text { ambient temperature }(\mathrm{K})
\end{aligned}
$$

\section{$\underline{\text { PM Loss Estimates }}$}

At low PM emission levels (2007 standard), submicron $(<100 \mathrm{~nm})$ PM particles will be the main content of PM particles [45]. For particles in this range, particles losses occur primarily due to diffusion $[35,157]$. This model's theoretical loss estimates for PM particles will help to determine the measured PM quantity. The equations used in this model for PM particle transport efficiency were previously presented by Ayala and Olson $[157,158]$. Their analysis is reproduced below:

$$
\begin{aligned}
& \eta_{\text {pipe }}=e^{-\zeta S h} \\
& S h=3.66+\frac{0.2672}{\zeta+0.10079 \zeta^{1 / 3}} \quad \text { (laminar flow) } \quad \text { (turbulent flow) } \\
& S h=0.0118 \operatorname{Re}^{7 / 8} S c^{1 / 3} \quad \mu \\
& S c=\frac{\mu}{\rho D} \\
& \zeta=\pi D L / Q
\end{aligned}
$$

Equation 4. 9

Equation 4. 10

Equation 4. 11

Equation 4. 12 


$$
D=\left(\frac{K T}{3 \pi \mu d_{p}}\right)\left(1+\frac{15.39}{P d_{p}}+7.518 e^{-0.0741 P d_{p}}\right)
$$

Equation 4. 13

where

$$
\begin{aligned}
& \eta_{\text {pipe }}=\text { particle penetration efficiency } \\
& \mathrm{Sh}=\text { Sherwood number } \\
& \mathrm{Sc}=\text { Schmidt number } \\
& \mu=\text { dynamic viscosity }(\mathrm{Pa} \cdot \mathrm{s}) \\
& \rho=\text { density }\left(\mathrm{kg} / \mathrm{m}^{3}\right) \\
& \mathrm{D}=\text { particle diffusion coefficient } \\
& \mathrm{Q}=\text { volumetric flow rate through the tunnel }\left(\mathrm{m}^{3} / \mathrm{min}\right) \\
& \mathrm{K}=\text { Boltzmann constant: } 1.3806503 \times 10^{-23} \mathrm{~m}^{2} \mathrm{~kg} \mathrm{~s}^{-2} \mathrm{~K}^{-1} \\
& \mathrm{~T}=\text { absolute air temperature }(\mathrm{K}) \\
& \mathrm{P}=\text { pressure }(\mathrm{Pa}) \\
& d_{p}=\text { particle diameter. }(\mathrm{m})
\end{aligned}
$$

Small particles attach to the tunnel walls, decreasing particle concentration near the walls, and creating a particle concentration gradient. Particles in the bulk flow then diffuse toward the wall because Brownian motion causes local mixing and effectively moves particles toward the lower concentration zone [157]. Those particles attached to the walls will accumulate, grow to larger sizes, and then be sheared off of the walls if a layer with weak mechanical properties forms. In legacy PM measurement systems, it was common to see large particles, that were shed from tunnel walls, on filters. For 2007 systems, most large particles will be removed by the cyclone ahead of the PM filter [35, 37, 38]. However, some of them will increase the measured PM data's variation. Some deposition on tunnel walls is by volatile vapor that condenses or adsorbs onto the wall and can desorb later $[45,150]$. Hydrocarbon hangup was demonstrated experimentally by Clark et al. [159]. It is important to make tunnels as short as possible within the constraint of 2007 regulations to reduce PM diffusion losses [45].

\section{Filter Face Velocity}

High filter face velocity results in less organic carbon trapped on the filter, and hence lower measured PM emissions [107, 160, and 161]. However, changes of measured 
PM emissions with higher or lower filter face velocity are also affected by what kind of engine was running and whether it was equipped with a DPF [35]. The filter face velocity is more likely to affect the mass of PM captured if the PM is low in elemental carbon content, which is likely to be true for post 2007 engines [35]. To simplify the effects modeling of filter face velocity on measured PM mass for FTP transient runs, when increasing filter face velocity from $20 \mathrm{~cm} / \mathrm{sec}$ to $100 \mathrm{~cm} / \mathrm{sec}$, there will be a $15 \%$ decrease for measured PM emissions [35].

\section{Filter Face Temperature}

Since the 2007 regulations will narrow the filter face temperature to a range of $10^{\circ} \mathrm{C}$, the effect of filter face temperature on measured PM mass was not modeled even though higher filter face temperature usually results in less trapped organic carbon [35, 107]. The model in this dissertation is actually used to see the variation of the filter temperature within the constraint of 2007's regulations.

\section{Real Time PM}

Real time PM emissions can be correlated well with $\mathrm{CO}$ emissions for certain conventional older engines [162]. Real time PM emissions from the engine in $\mathrm{g} / \mathrm{sec}$ were estimated using real time CO emissions by the following empirical relationship [163].

$$
P M_{\text {realtime }}=C O_{\text {realtime }} \frac{P M_{\text {total }}}{C O_{\text {total }}}
$$

Equation 4. 14

Real time $\mathrm{CO}$ emissions, total PM mass and total $\mathrm{CO}$ mass data were obtained from an FTP run of a 2004 Cummins ISM370 engine which was not trap equipped. Real time PM mass deposited on the filter then can be calculated with corresponding dilution ratios.

\subsubsection{Simulation Variables}

This parametric study was conducted by varying engine running conditions and geometric sizes of the PM measurement system. The heavy-duty engine used in this model was held constant to a 2004 Cummins ISM370 engine. To investigate the possible 
impact of proposed alternative PM measurement systems, the following variables were defined:

Engine air fuel ratio: $\mathrm{A} / \mathrm{F}$

Engine exhaust temperature: $T_{e}(\mathrm{~K})$

Engine exhaust flow rate: $V_{e}\left(\mathrm{~m}^{3} / \mathrm{sec}\right)$

Tunnel inner diameter (ID)*: $D_{t}, D_{p}, D_{s}(\mathrm{~cm})$

Tunnel length: $L_{t}, L_{p}, L_{s}(\mathrm{~m})$

Tunnel wall thickness: $\delta_{t}, \delta_{p}(\mathrm{~mm})$

Tunnel outer surface insulation thickness: $\delta_{t i}, \delta_{p i}(\mathrm{~cm})$

Tunnel inner surface temperature: $T_{t}, T_{p}, T_{s}(\mathrm{~K})$

Tunnel flow rate: $V_{p}\left(\mathrm{~m}^{3} / \mathrm{sec}\right), V_{s}($ Liter $/ \mathrm{sec})$

Secondary dilution ratio:

*subscripts: t(exhaust transfer pipe); p(primary tunnel); s(secondary tunnel)

Ambient air temperature was set to $27^{\circ} \mathrm{C}$. Filtered dilution air temperature for the secondary tunnel was set to $38^{\circ} \mathrm{C}$. This value is required to be equal to or greater than $15^{\circ} \mathrm{C}$ by the CFR [38]. The tunnels' wall material was constrained to stainless steel AISI 304 with density $7900 \mathrm{~kg} / \mathrm{m}^{3}$ and heat capacity $477 \mathrm{~J} /(\mathrm{kg} \cdot \mathrm{K})$. Mineral fiber foam with conductivity $0.035 \mathrm{~W} /(\mathrm{m} \cdot \mathrm{K})$ and emittance coefficient 0.85 was used to insulate the transfer pipe and the primary tunnel. A baseline model with the following assigned values was set up to discuss the influence of variables:

Tunnel ID: $D_{t}=12.7, D_{p}=50.0, D_{s}=1.0(\mathrm{~cm})$

Tunnel length: $L_{t}=5.0, L_{p}=7.0, L_{s}=1.2(\mathrm{~m})$

Tunnel wall thickness: $\delta_{t}=2.0, \delta_{p}=5.0(\mathrm{~mm})$

Tunnel insulation thickness: $\delta_{t i}=2.0, \delta_{p i}=5.0(\mathrm{~cm})$

Initial tunnel temperature: $T_{t}=376, T_{p}=304, T_{s}=320(\mathrm{~K})$

Tunnel flow rate: $V_{p}=1.0\left(\mathrm{~m}^{3} / \mathrm{sec}\right), V_{s}=1.0($ liter/sec $)$ 
Secondary dilution ratio: $D R_{s}=2.0$

Set values for the transfer pipe and primary tunnel temperatures were initial tunnel wall temperatures when the simulation begun. They were obtained when the engine was running at idle. The secondary tunnel was heated and insulated over the entire length to maintain a constant wall temperature. Flow rates mentioned in this dissertation were all referred to standard condition.

This model was used to simulate under three engine running conditions: running at idle, running at full power, and running an FTP transient cycle. Real time data for air fuel ratio, exhaust temperature, and exhaust flow rate were recorded from an FTP run of a 2004 Cummins ISM370 heavy-duty engine. Even though this was not a 2007 engine, data recorded from its running were effective to test this system model.

\subsubsection{Model Validation}

While the 2007 standard PM measurement system at WVU was under construction, the model presented here was applied to a pre-existing PM measurement system to validate the predictions of the model for the new system. The simulated results were compared with the real world test results from a 2004 Cummins ISM370 HDD engine. An Orange County Transit Authority (OCTA) chassis test cycle was run with following variables:

Tunnel ID ${ }^{*}: D_{t}=12.7, D_{p}=50.0, D_{s}=2.22(\mathrm{~cm})$

Tunnel length: $L_{t}=5.0, L_{p}=7.0, L_{s}=2.13(\mathrm{~m})$

Tunnel wall thickness: $=2.0, \delta_{p}=5.0(\mathrm{~mm})$

Tunnel insulation thickness: $\delta_{t i}=2.0, \delta_{p i}=5.0(\mathrm{~cm})$

Initial tunnel temperature: $T_{t}=370, T_{p}=302(\mathrm{~K})$

*subscripts: t(exhaust transfer pipe); p(primary tunnel); s(secondary tunnel)

Continuous real time data used in the model simulation included the engine exhaust temperature and flow rate, the secondary tunnel flow rate and dilution ratio, and 
the primary flow rate. The primary dilution air temperature was set to $35^{\circ} \mathrm{C}$ and the secondary dilution air temperature was set to $20^{\circ} \mathrm{C}$. A CFV-CVS was employed instead of a SSV-CVS in this pre-existing system. There was an $8.0 \mathrm{~m}$ long un-insulated aluminum pipe connecting the primary tunnel and the CFV, having an inner diameter of $0.3 \mathrm{~m}$ and a wall thickness of $2.0 \mathrm{~mm}$. Figure 31 illustrates the simulated and real-world measured temperatures at the inlet of the CFV. Figure 32 shows the simulated and the measured filter face temperatures.

As shown in Figure 31, the engine exhaust temperature increased from around $150^{\circ} \mathrm{C}$ to $250^{\circ} \mathrm{C}$, but only a slight increase for the simulated and the measured venturi temperatures was observed. The simulated venturi temperature had higher peaks than the measured one. This indicates that the real flow could exchange more energy with the measurement system than the simulated flow. The response time of the Resistant Temperature Detector (RTD) for the experimental data may also play a role. The average absolute error for the simulated venturi temperature compared with the measured venturi temperature was $7.6 \%$ of the measured venturi temperature. Since the heat transfer equations used for conduction were empirically derived, some deviation between the model and measurements was expected.

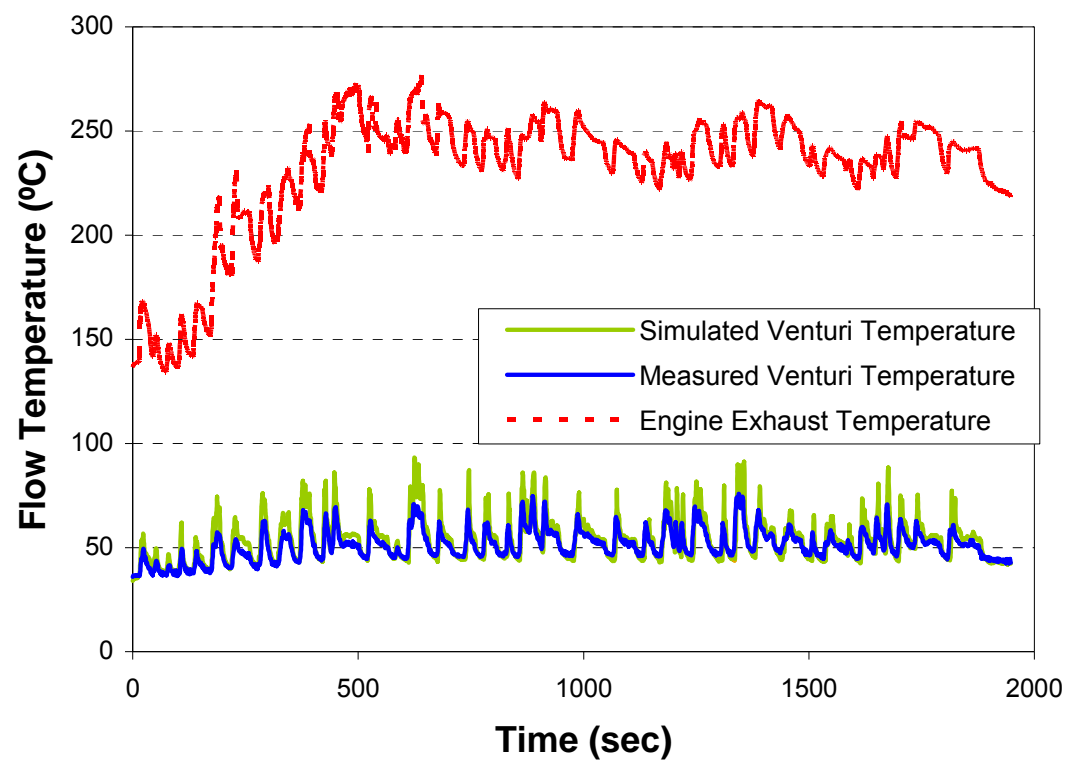

Figure 31 Simulated and measured CFV inlet temperatures 


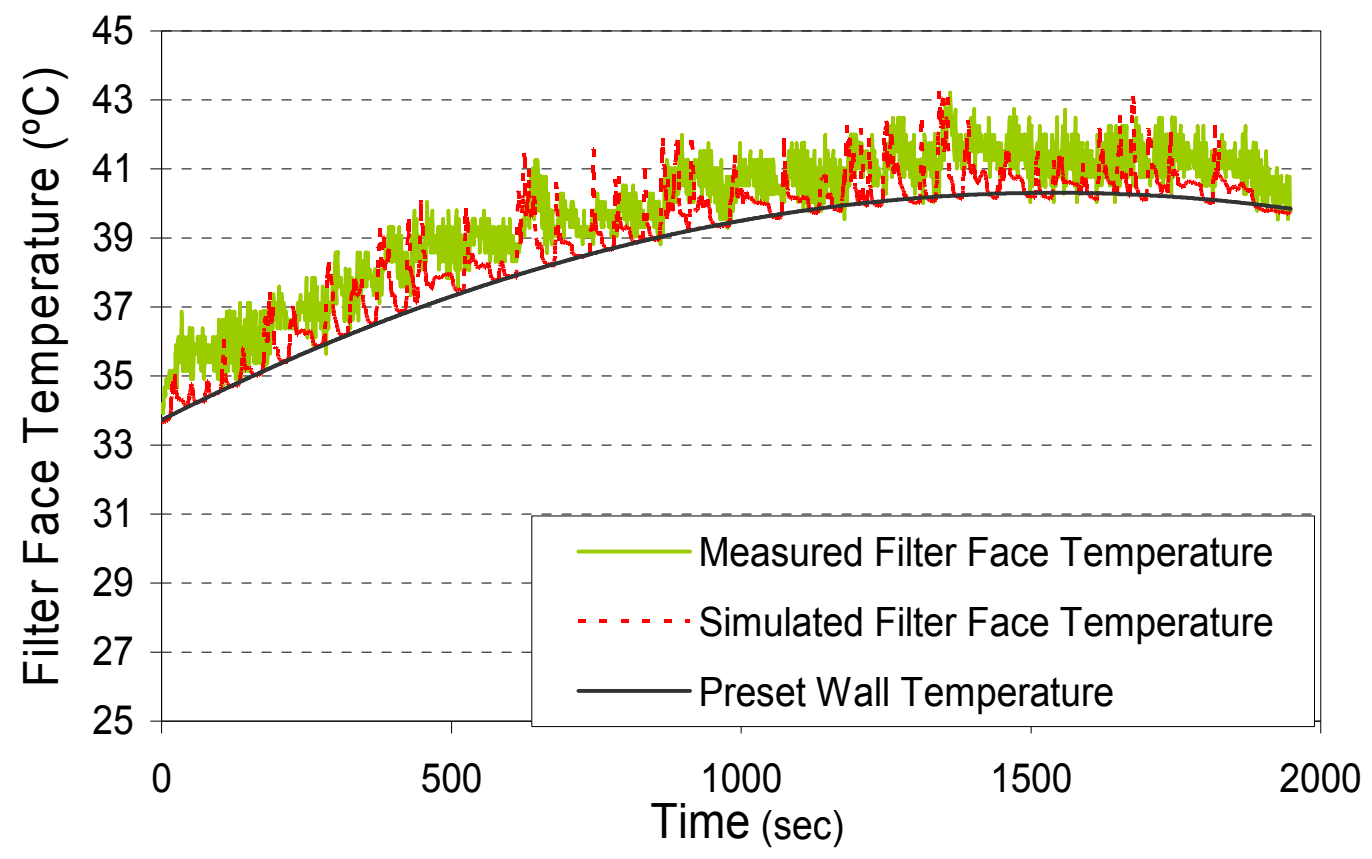

Figure 32 Simulated and measured filter face temperatures

Figure 32 presents the simulated filter face temperature with a pre-set wall temperature curve and the measured filter face temperature variation with time. The secondary tunnel's wall temperature was not held at a constant value. Both the simulated and measured filter face temperatures followed the wall temperature and had the same temperature fluctuation of $3^{\circ} \mathrm{C}$. The measured filter face temperature curve showed oscillations, but both Figure 31 and Figure 32 show that the simulated venturi and filter face temperatures correlated with real-world measured ones. The good agreements of the model with existing data have built confidence in the model's predictive ability for the new tunnel design.

\subsubsection{Simulation Results}

The impact of variables was investigated by altering variable values of the baseline model individually in the following categories. Attention was mainly focused on the filter face temperatures and the estimated PM diffusion losses as predicted by the model of Ayala and Olson. Effects of total dilution ratios on the cumulative filter PM mass were also discussed. To simplify stating variable values, all variables other than the variable concerned were the same as the baseline model if not specifically mentioned. 


\section{Effect of Transfer Pipe Size}

Filter face temperatures were investigated as the transfer pipe length changed from $1.0 \mathrm{~m}$ to $6.0 \mathrm{~m}$ while a standard 5 inch $(12.7 \mathrm{~cm})$ ID pipe was used. Figure 33 shows the maximum and minimum filter face temperatures for an FTP test with different transfer pipe lengths. Figure 34 shows the estimated average PM loss through the transfer pipe for an FTP test. A secondary tunnel with diameter of $2.0 \mathrm{~cm}$ and length of $1.0 \mathrm{~m}$ was also investigated in Figure 33.

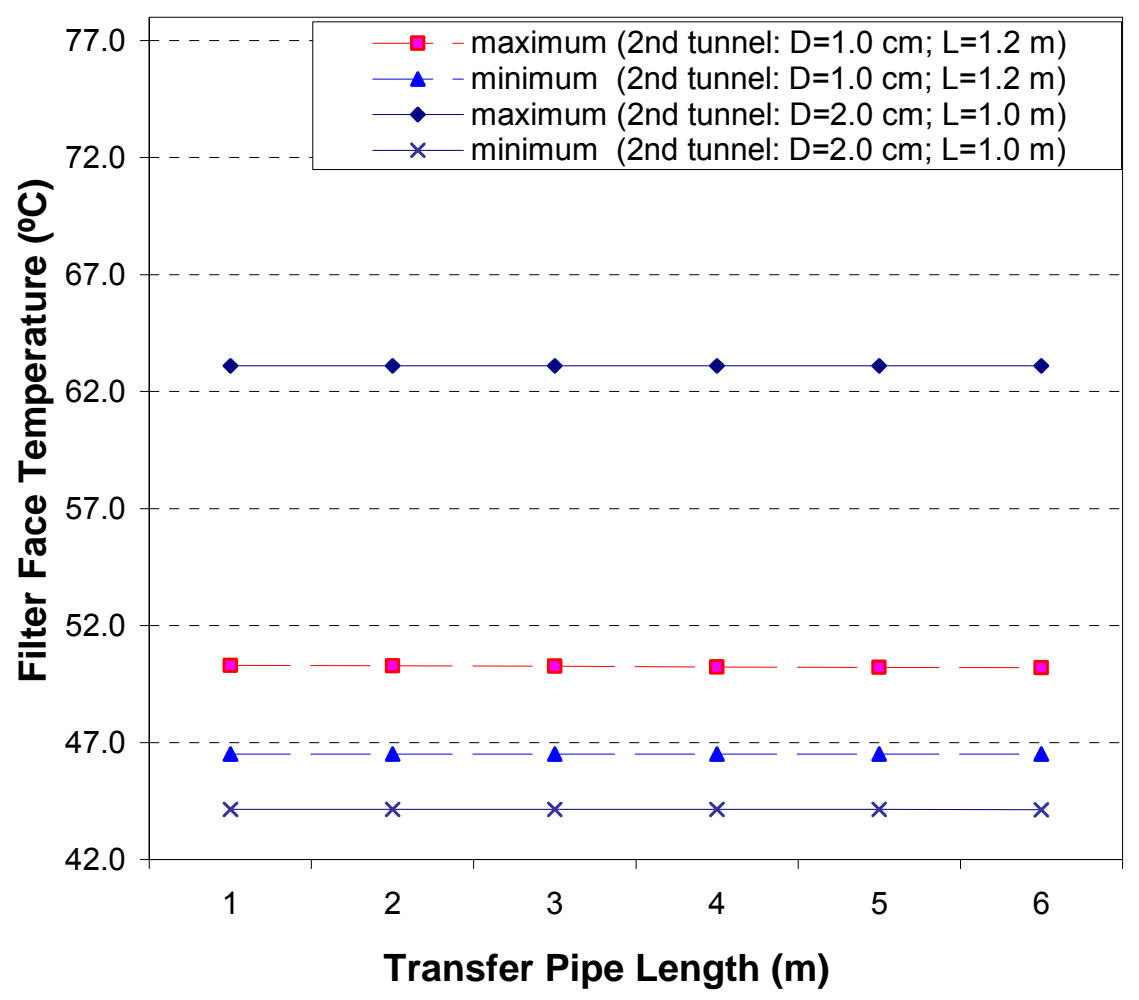

Figure 33 Maximum and minimum filter face temperatures with different transfer pipe lengths

Figure 33 illustrates that changing the transfer pipe length had little effect $(<$ $0.2^{\circ} \mathrm{C}$ of change) on filter face temperatures. For the $1.0 \mathrm{~m}$ long secondary tunnel, the maximum filter face temperature did not meet the 2007 standards. The filter maximum and minimum face temperatures for both secondary tunnel configurations showed negligible change as the transfer pipe length increased. Minimal change $\left(<0.2^{\circ} \mathrm{C}\right.$ of change) was also observed for the exhaust temperature at the inlet of the secondary tunnels. 
Figure 34 presents the average PM particle penetration efficiency distributions through the transfer pipe for different pipe lengths. As expected, longer pipes had lower penetration efficiency for PM particles and caused more particle diffusion losses. For particles with diameters larger than $10 \mathrm{~nm}$, there were almost no losses $(>97 \%$ penetration efficiency) due to particle diffusion, and this implies that there would be negligible mass lost to the pipe walls. For particles with diameters less than $10 \mathrm{~nm}$, a shorter pipe can significantly reduce the particle diffusion losses.

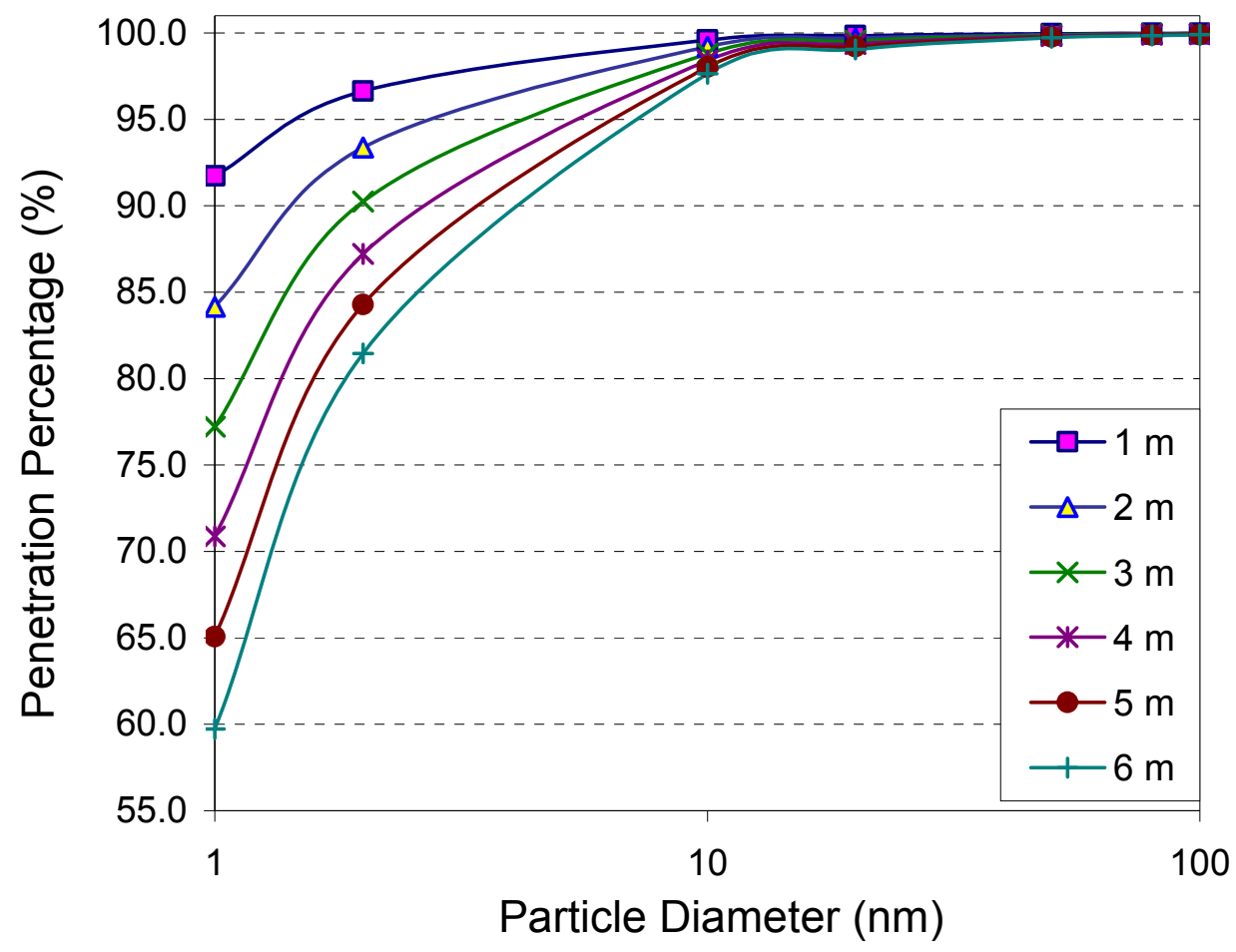

Figure 34 PM penetration percentage distributions with different transfer pipe lengths

\section{Effect of Primary Tunnel Size}

Both the diameter and length of the primary tunnel were changed to see their effects with two secondary tunnel configurations shown in Figure 7. The model was run for the primary tunnel lengths from $5.0 \mathrm{~m}$ to $15.0 \mathrm{~m}$ and for inner diameters from $20.0 \mathrm{~cm}$ to $70.0 \mathrm{~cm}$. The maximum and minimum filter face temperatures maintained similar values. Changing the size of the primary tunnel had little effect on the filter face temperature. 
PM diffusion losses through the primary tunnel showed the same pattern as Figure 34 displays with different tunnel lengths. PM diffusion losses for particles with diameters greater than $10 \mathrm{~nm}$ were negligible. PM losses for smaller particles increased with the increment of the tunnel length and decreased with the increment of the tunnel inner diameter. A primary tunnel with length of $15.0 \mathrm{~m}$ and $50.0 \mathrm{~cm} \mathrm{ID}$, or a primary tunnel with length of $7.0 \mathrm{~m}$ and $20.0 \mathrm{~cm}$ ID, had a similar PM penetration efficiency distribution with a $4.0 \mathrm{~m}$ long transfer pipe for an FTP run.

\section{Effect of Secondary Tunnel Size}

The secondary tunnel's inner diameter was changed from $5.0 \mathrm{~mm}$ to $40.0 \mathrm{~mm}$ and the length was increased from $0.5 \mathrm{~m}$ to $4.0 \mathrm{~m}$. The effect of changing the secondary tunnel size on the filter face temperatures are shown in Figure 35 and Figure 36. Modeling results of the real time flow temperatures at different locations of the PM measurement system are shown in Figure 37 for different secondary tunnel lengths. PM penetration efficiency distributions for different tunnel inner diameters are shown in Figure 38 .

Figure 35 shows the maximum and minimum filter face temperature for different secondary tunnel lengths. The minimum filter face temperature was always above the lower limit of the 2007 standard of $42^{\circ} \mathrm{C}$. However, the maximum filter face temperature exceeded the upper limit of $52^{\circ} \mathrm{C}$ for the 2007 regulation when the secondary tunnel was less than $1.0 \mathrm{~m}$ in length. When the length of the secondary tunnel was increased, maximum and minimum filter face temperatures closed rapidly to the preset wall temperature. Once the tunnel length reached $2.0 \mathrm{~m}$, filter face temperature stabilized around the preset wall temperature and no significant changes occurred with a longer secondary tunnel. 


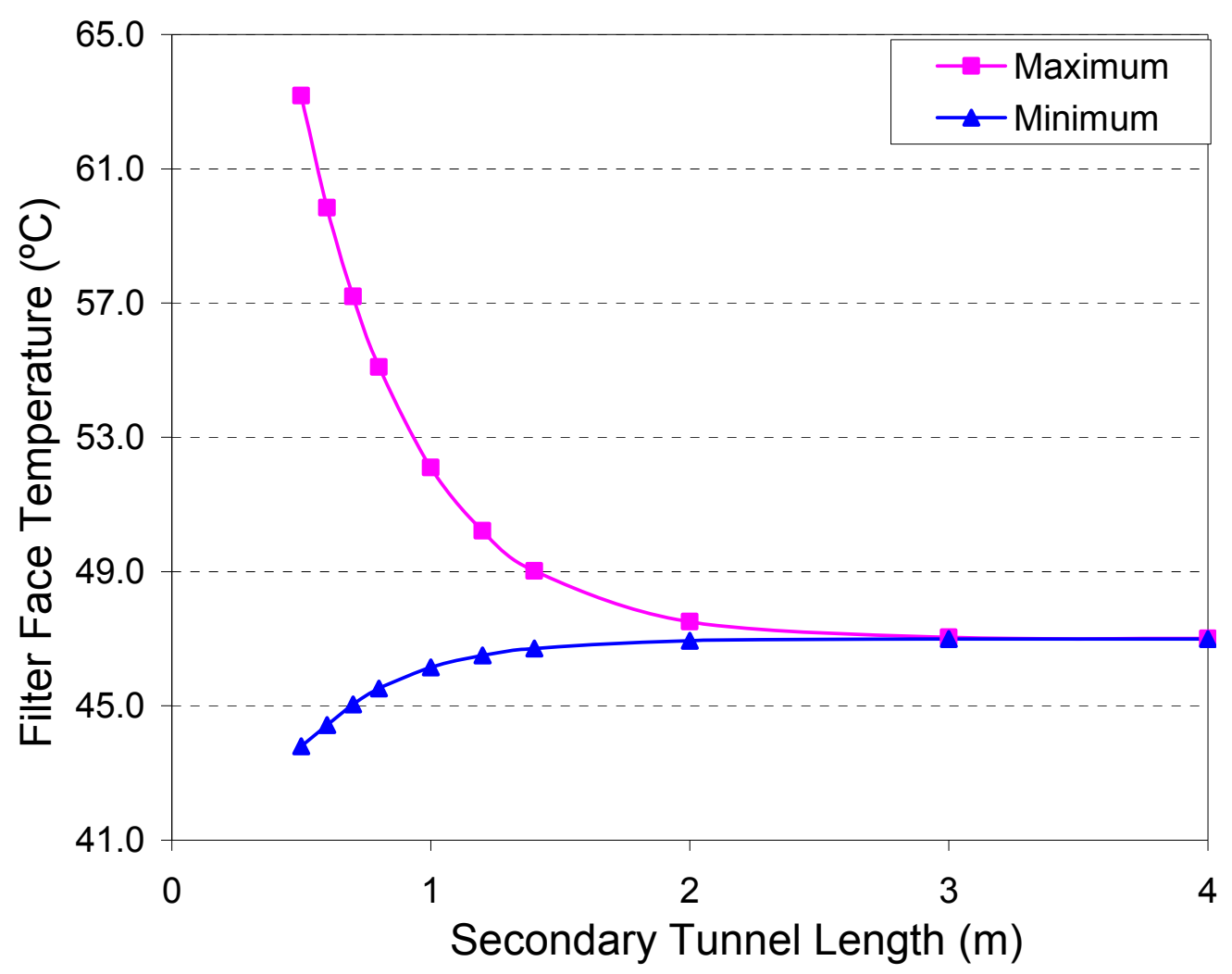

Figure 35 Maximum and minimum filter face temperatures with different secondary tunnel lengths

In Figure 36, filter face temperatures were shown with three different secondary tunnel length settings. The fluctuation of the filter face temperatures became smaller with a longer secondary tunnel. When the secondary tunnel was $0.5 \mathrm{~m}$ long, the filter face temperatures were not within the 2007 standard. The filter face temperatures of the $0.5 \mathrm{~m}$ long secondary tunnel fluctuated between $43.6^{\circ} \mathrm{C}$ and $62.1{ }^{\circ} \mathrm{C}$. The filter face temperatures of $4.0 \mathrm{~m}$ long secondary tunnel were almost steady, as shown in Figure 36.

Figure 37 summarizes the trends of the maximum and minimum filter face temperature when the secondary tunnel's diameter changed. Similar to changing of the secondary tunnel's length, changing diameter had a larger impact on the maximum filter face temperature than on the minimum filter face temperature. When the secondary tunnel's diameter was greater than $18.0 \mathrm{~mm}$, the filter face temperature was outside the limits of the 2007 standard. 


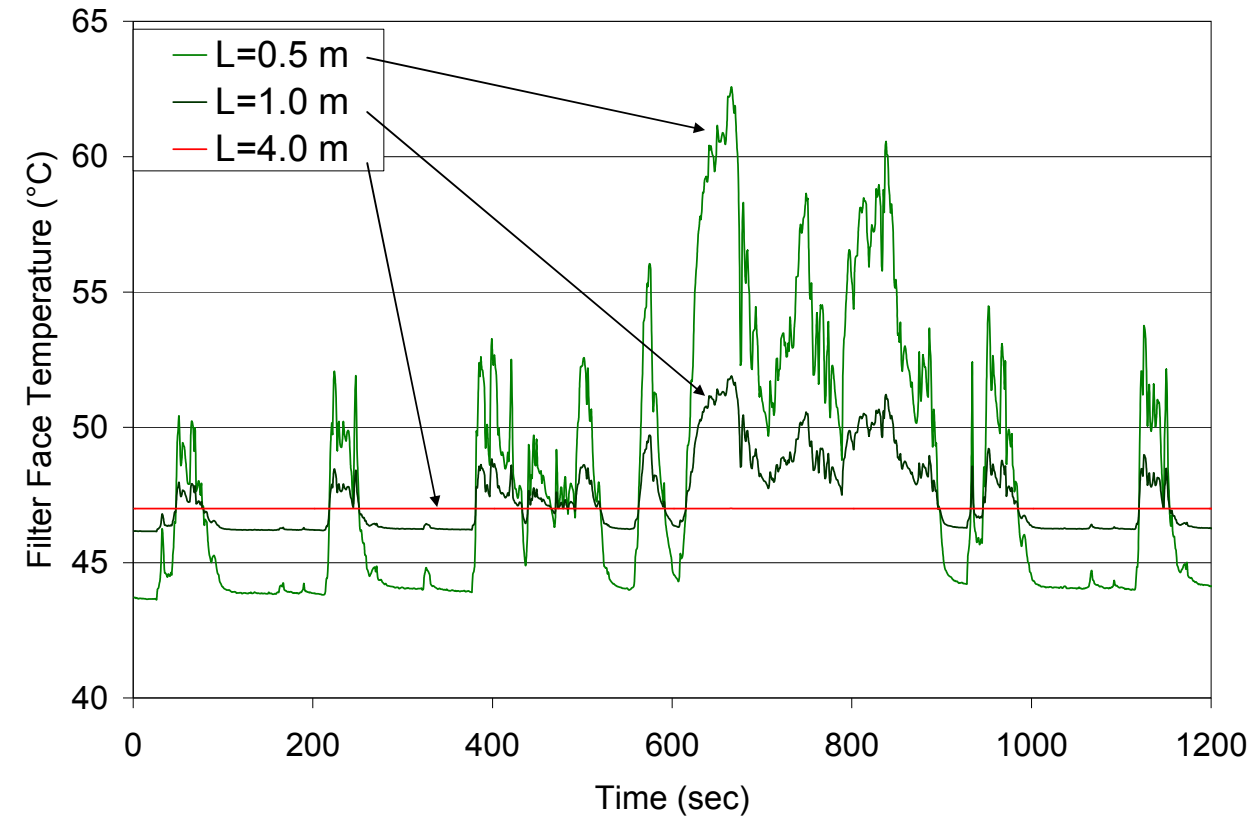

Figure 36 Real time filter face temperatures for an FTP run with different secondary tunnel lengths

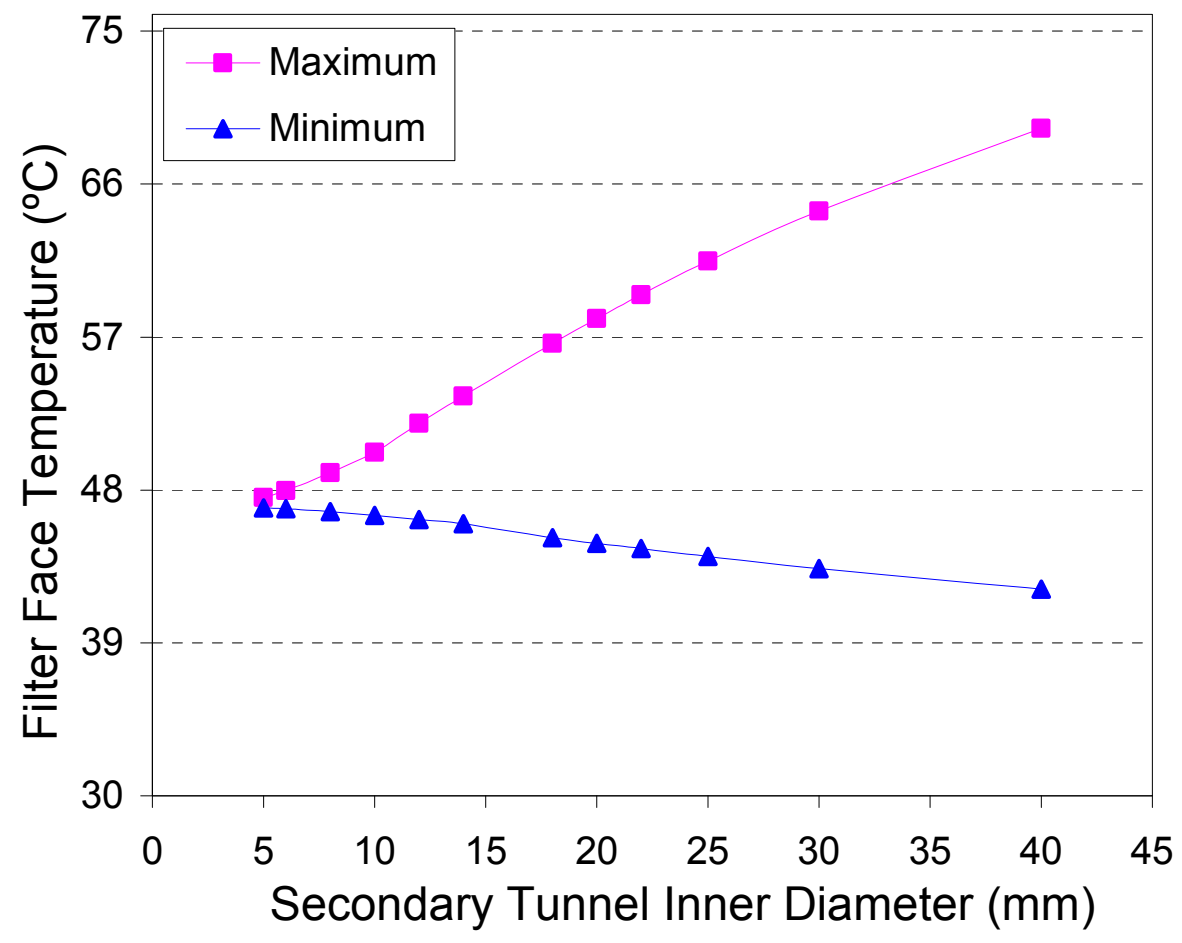

Figure 37 Maximum and minimum filter face temperatures with different secondary tunnel diameters 
Figure 38 presents PM penetration efficiency through the secondary tunnel for particles of different sizes when the secondary tunnel's inner diameter changes. The secondary tunnel with a smaller diameter had lower penetration efficiency for PM particles and caused more particle losses. For PM particles with diameters less than 10 $\mathrm{nm}$, penetration efficiency dropped dramatically as the secondary tunnel's diameter decreased. Model results also showed that longer secondary tunnels had more PM diffusion losses than expected. Compared to PM losses through the transfer pipe and the primary tunnel, PM losses through the secondary tunnel accounted for the major part of total PM diffusion losses throughout the system.

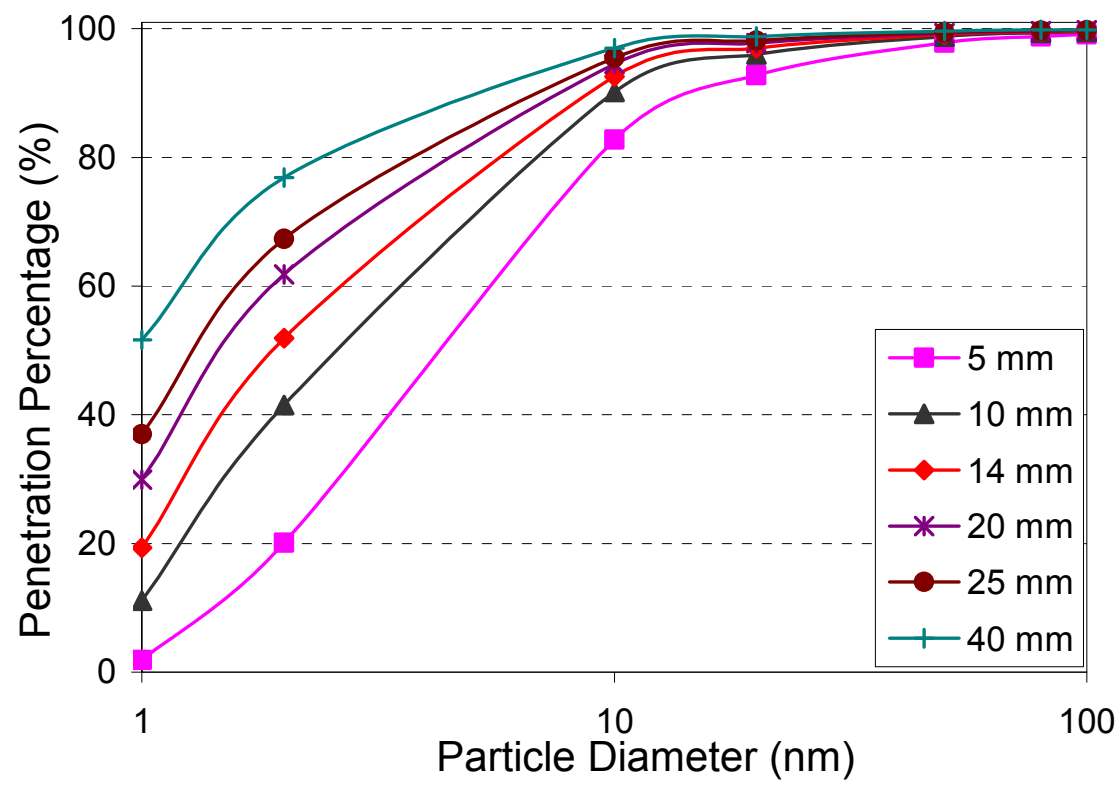

Figure 38 PM penetration percentages with different secondary tunnel diameters

\section{Effect of Insulation}

Insulation for the transfer pipe and the primary tunnel was optional for 2007 PM measurement systems but was highly recommended to minimize the heat losses outside of the system. Simulation results showed that insulation had little influence on both filter face temperature and PM diffusion losses, but did affect the system's heat losses. Total heat losses to the outside of the PM measurement system through convection and radiation for an FTP test were examined for different radiation insulation thickness of the transfer pipe and the primary tunnel. The results are illustrated in Figure 39. 


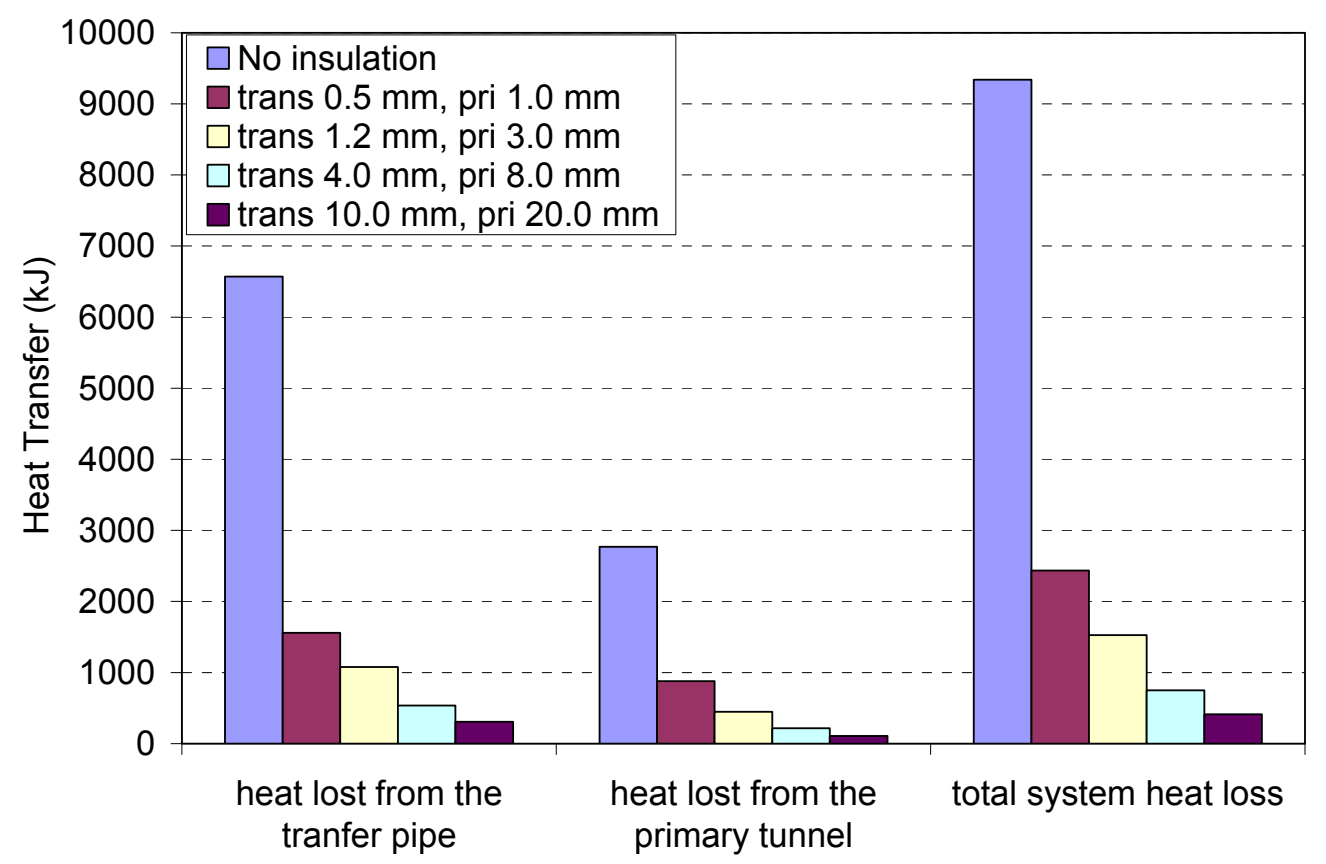

Figure 39 Heat lost to the outside of the measurement system with different thickness of insulation

Figure 39 demonstrates that insulation can substantially reduce the measurement system heat loss. Compared with pipes that had no insulation, the system with only 0.5 $\mathrm{cm}$ thick insulation for the transfer pipe and $1.0 \mathrm{~cm}$ thick insulation for the primary tunnel could reduce system heat losses by $75 \%$. If the transfer pipe had more than $4.0 \mathrm{~cm}$ thick insulation and the primary pipe had more than $8.0 \mathrm{~cm}$ thick insulation, the system's heat loss could be reduced by more than $92 \%$ compared to losses with no insulation.

Cumulative heat losses with different thickness of insulation were compared with cumulative exhaust energy delivered to the PM measurement system. Results are plotted in Figure 40.

Figure 40 shows approximately $60 \mathrm{MJ}$ of total exhaust energy was delivered by the engine to the PM measurement system for an FTP run. If there was no insulation for the transfer pipe and the primary tunnel, total heat loss would account for $15.6 \%$ of total exhaust energy. Less total heat loss occurred with thicker insulation for the transfer pipe and the primary tunnel. 


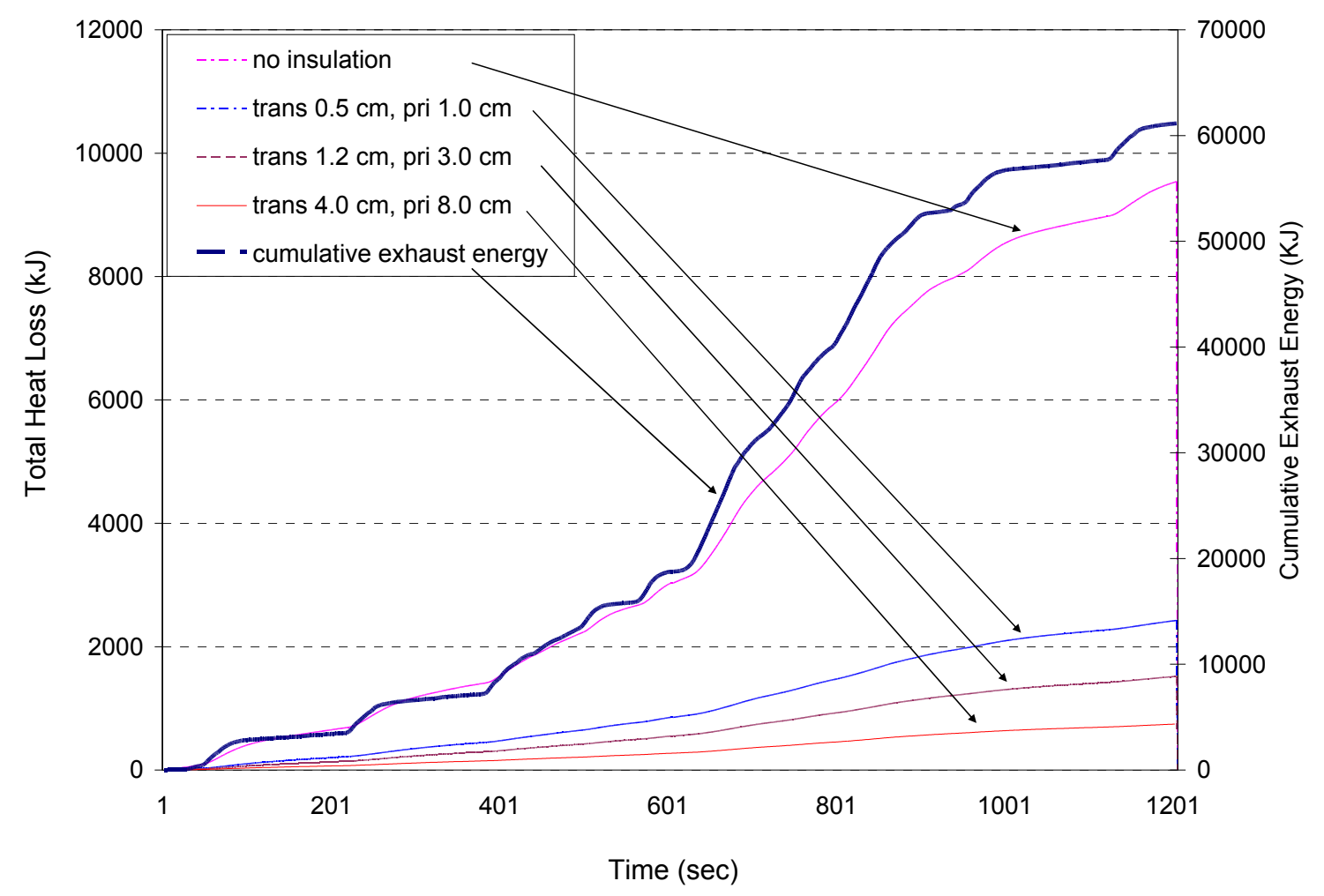

Figure 40 Cumulative total heat lost to the outside of the measurement system with different thickness of insulation and the cumulative exhaust energy delivered by the engine

\section{Effect of Primary Tunnel Flow}

Engine exhaust was supposed to be fully mixed at the end of primary tunnel before being diluted a second time. A turbulent flow (Reynolds Number greater than 4000) inside the primary tunnel was required to ensure the complete mixing of the exhaust and dilution air by the CFR [38]. The temperature of the primary dilution air was required to be equal to or greater than $15^{\circ} \mathrm{C}$, which is set to be $27^{\circ} \mathrm{C}$ here. Figure 41 shows the changes of the filter face temperatures with different primary flow rates. Modeling results of the real time filter face temperatures are shown in Figure 42 for different primary tunnel flow rates.

Figure 41 illustrates that the maximum filter face temperature dropped from above the 2007 upper limit to below the upper limit when the primary flow rate increased. However, the minimum filter face temperature was almost constant due to the low temperature of engine exhaust at idle. When the engine exhaust was at high 
temperature, low primary flow rates would result in small dilution ratios and the high temperature of exhaust could not be reduced after being diluted. Then, the high secondary inlet exhaust temperature caused the maximum filter face temperature to become too high. A primary flow rate of greater than $0.7 \mathrm{~m}^{3} / \mathrm{sec}$ was required to maintain the filter face temperatures within the constraint of 2007 regulations. Calculations also showed growing primary flow could slowly increase the PM diffusion losses but such a change would be minimal.

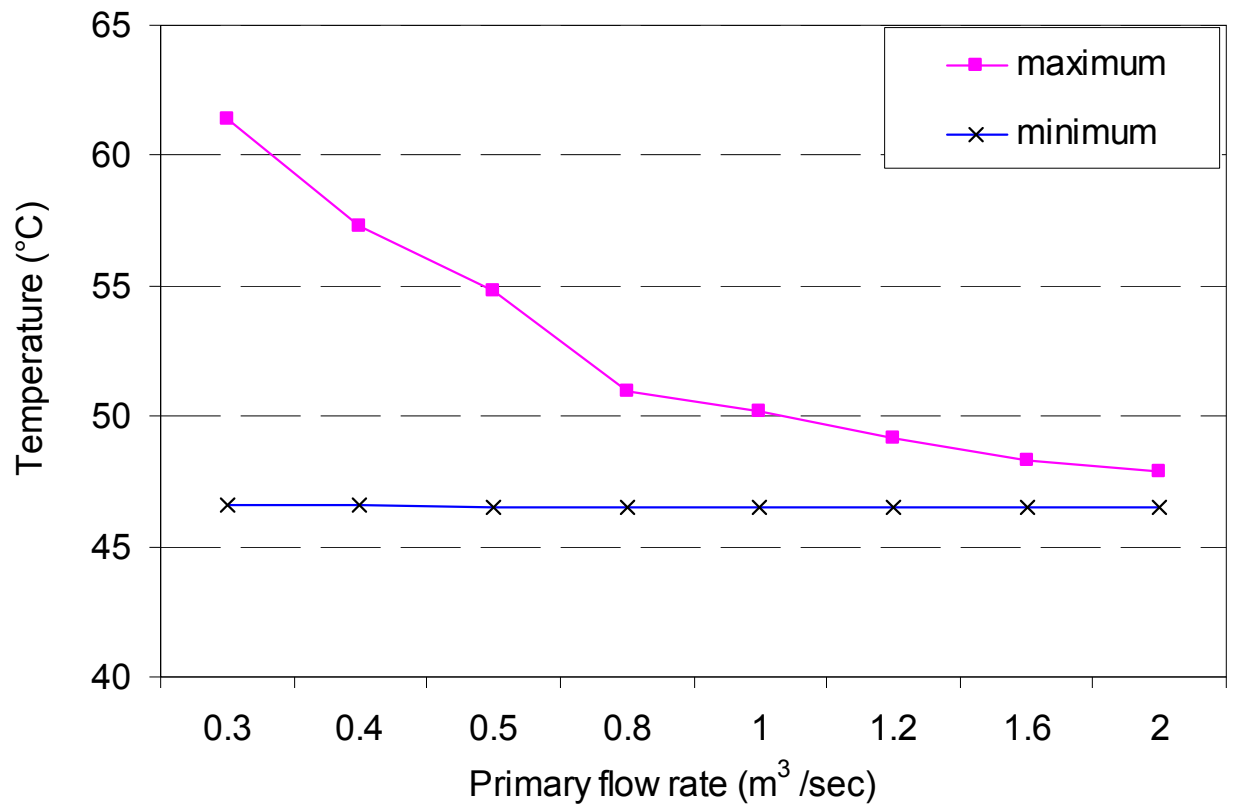

\section{Figure 41 Maximum and minimum filter face temperatures with different primary flow rates}

Three different primary flow rates: $0.4,1.0$ and $2.0 \mathrm{~m}^{3} / \mathrm{sec}$ were investigated and three corresponding filter face temperatures were shown in Figure 42. The filter face temperatures were more variable with lower primary flow rates. The flow at the end of the primary tunnel was more cooled with higher primary flow rates due to dilution of exhaust. Theoretically, the flow temperatures at the end of the primary tunnel could be maintained within $47^{\circ} \mathrm{C} \pm 5^{\circ} \mathrm{C}$, given a high enough primary flow rate with a proper primary dilution air temperature. When the primary dilution air temperature was set to $43^{\circ} \mathrm{C}$, simulations showed that the primary flow needed to the at least $15.0 \mathrm{~m}^{3} / \mathrm{sec}$ so that 
no secondary flow was required. Such a high primary flow rate is infeasible for a real world system. It is impractical to meet the 2007 filter face temperature regulations merely by changing the primary dilution air temperature and the primary flow rate.

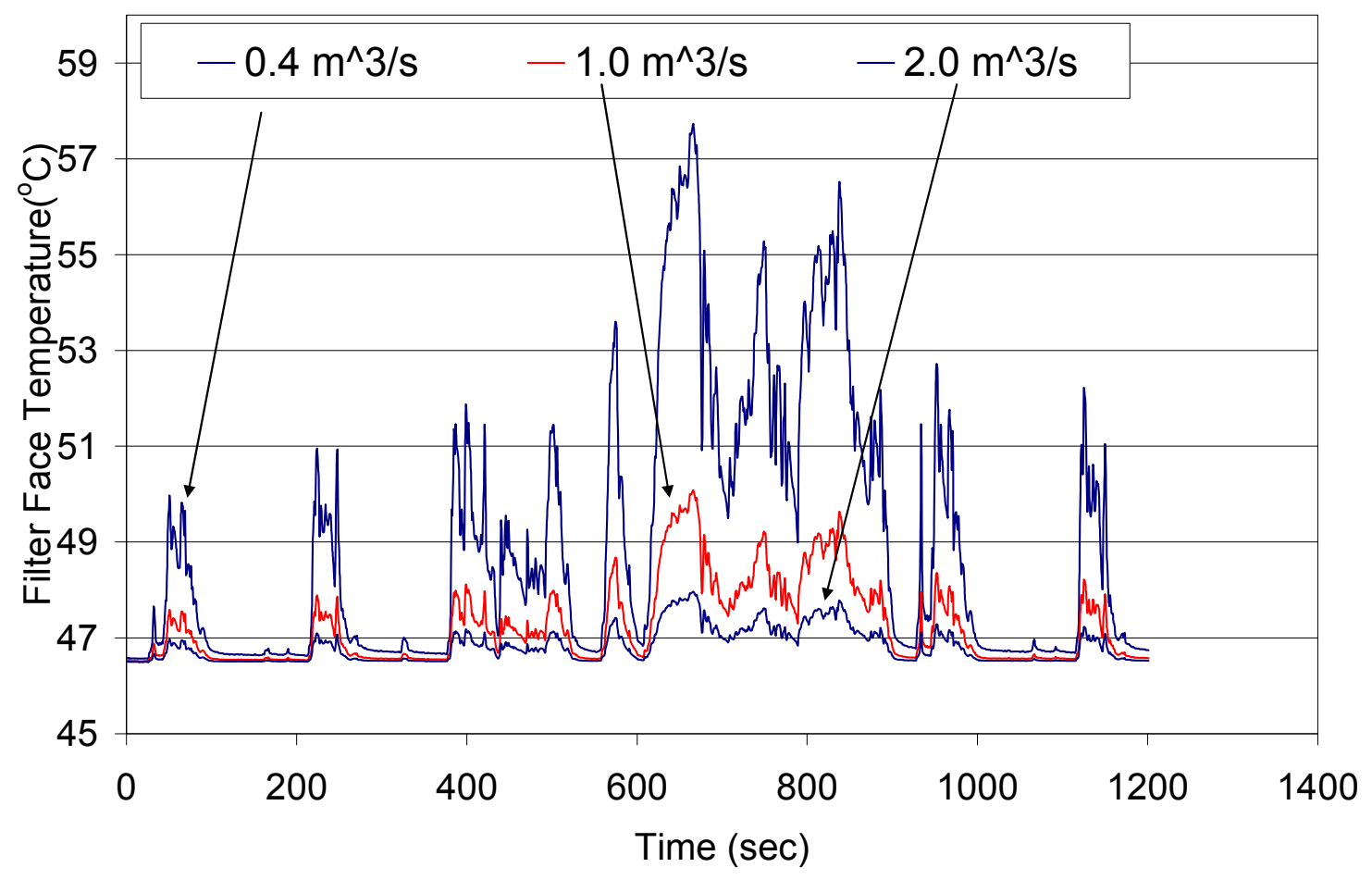

Figure 42 Real time filter face temperatures with different primary flow rates

\section{Effect of Enqine Running Condition}

To see the impact of the PM measurement system's transient thermodynamic responses on the filter face temperature, simulations of engine running at idle, at full power, and switching between running at idle and at full power with different frequencies were performed. Figure 43 shows the maximum and minimum filter face temperatures for different engine running conditions.

The engine was running at idle and was switched to full power then went back to idle for every 10 seconds, 1 minute, 2 minutes, 5 minutes and 20 minutes. Simulations showed that, for two different secondary tunnel configurations, the maximum and minimum filter face temperatures deviated slightly from each other with lower switching frequency of the engine running condition. 


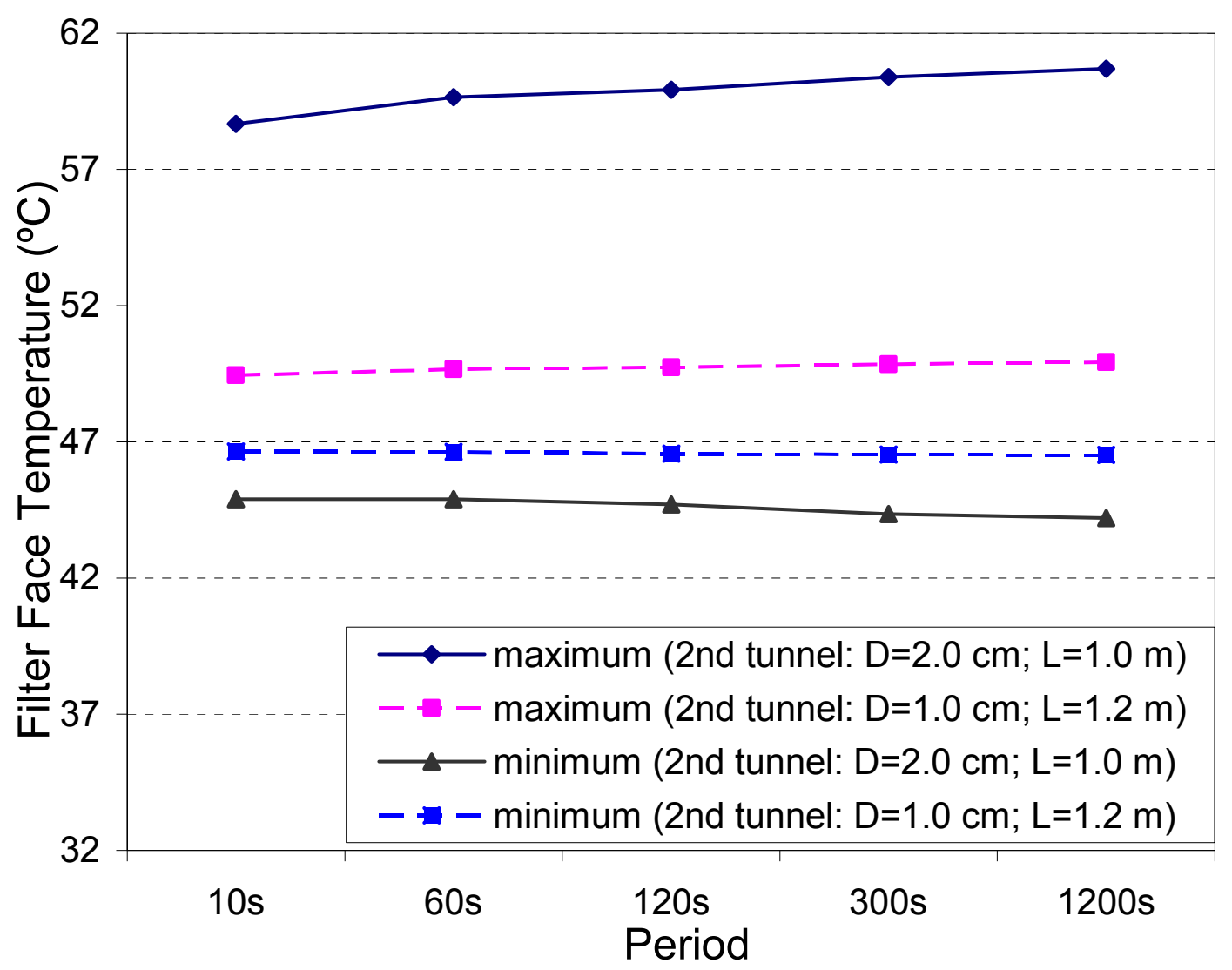

Figure 43 Maximum and minimum filter face temperatures when the engine was running between at idle and at full power

\section{Effect of Secondary Tunnel Flow}

As described in CFR, the filter face velocity is required to be less than 100.0 $\mathrm{cm} / \mathrm{sec}$. For a $47 \mathrm{~mm}$ filter with $38.5 \mathrm{~mm}$ stain diameter, the allowed maximum flow rate for the secondary tunnel was around 1.2 liters per second. Different secondary tunnel flow rates were simulated to investigate the effects on the filter face temperature. The results are shown in Figure 44. Except the secondary tunnel flow rate, all variable values were held constant at the values of the baseline model. The secondary dilution ratio was a constant of 2.0. The overall dilution ratios for the secondary flow were always 2.0 times of the dilution ratios for the primary flow. Dilution ratios for the primary flow are shown in Figure 30. 


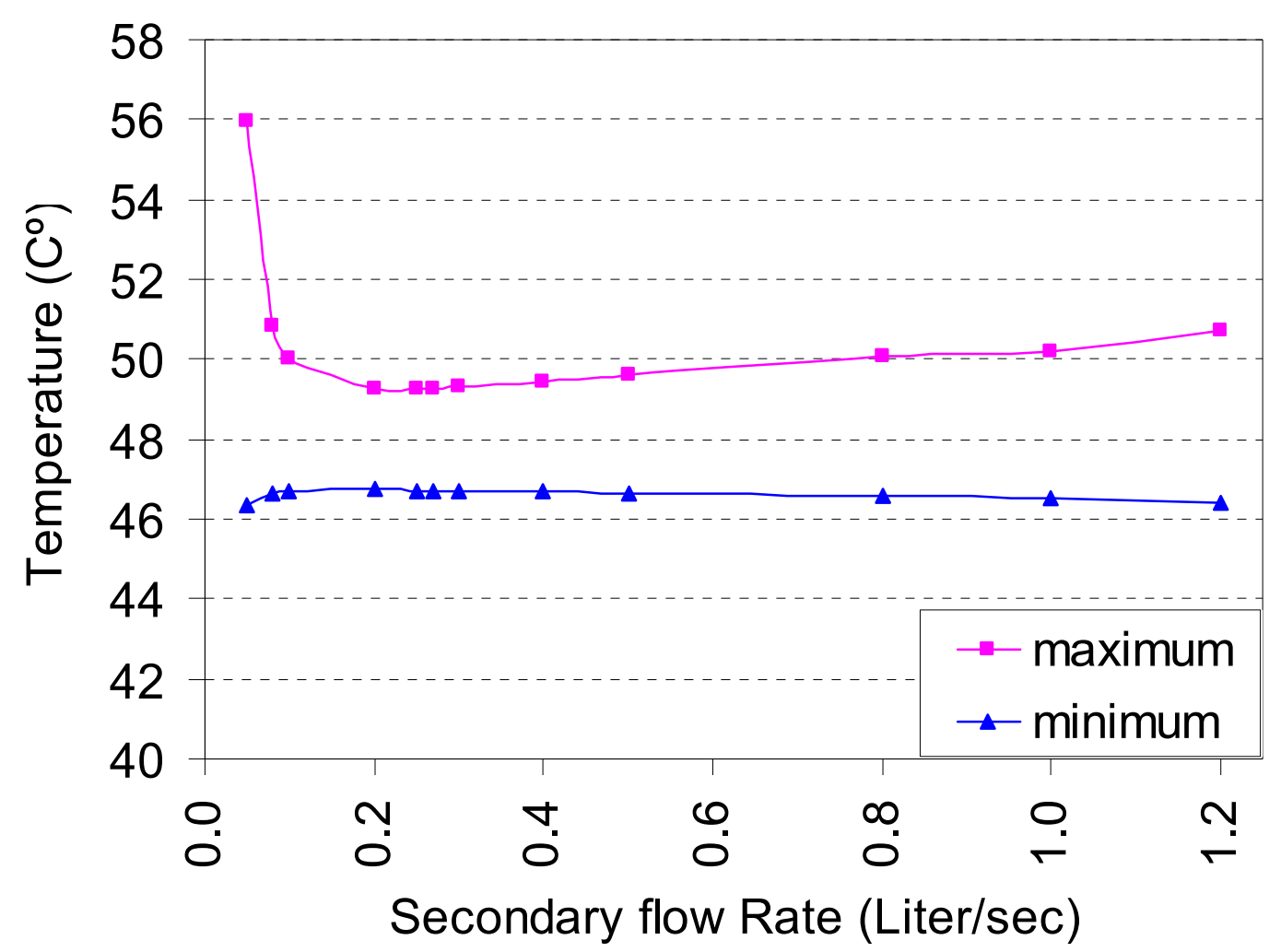

Figure 44 Maximum and minimum filter face temperatures with different secondary tunnel flow rates

As shown in Figure 44, the maximum filter face temperature drops first, then goes up with the increasing secondary flow rate; while the minimum filter face temperature rises first then goes down from low to high secondary flow rate. At low flow rates, the secondary tunnel was at a laminar condition and the convection heat transfer between the tunnel wall and the exhaust was weak. As a result, the temperature did not change much. As the flow rate increased, the secondary flow transitioned to turbulent flow and the convection heat transfer became stronger. The flow's temperature narrowed to the wall's temperature. As the secondary flow rate was further increased, the heat transfer between the tunnel wall and the flow was also further enhanced. However, once the flow rate reached a certain point $(0.25 \mathrm{~L} / \mathrm{sec})$, the increasing rate of the flow mass became higher than the increasing rate of total heat transferred. As a result, the maximum and the minimum filter face temperature started deviating from each other. 


\section{Effect of Preset Secondary Tunnel Wall Temperature}

The secondary tunnel was modeled as being insulated and heated to maintain the controlled tunnel wall temperature. Figure 45 shows the effect of the preset secondary tunnel temperature on the filter face temperature.

Figure 45 examined the trends of the maximum and the minimum filter face temperatures with different preset secondary wall temperatures. As Figure 45 shows, the maximum and the minimum filter face temperatures were linear with respect to the preset wall temperatures. The minimum filter face temperature was close to the preset wall temperature and the maximum filter face temperature was always 3.5 to $4.0^{\circ} \mathrm{C}$ higher than the minimum filter face temperature. Calculations also showed that there were minimal effects for small changes of the preset wall temperature on the PM diffusion losses.

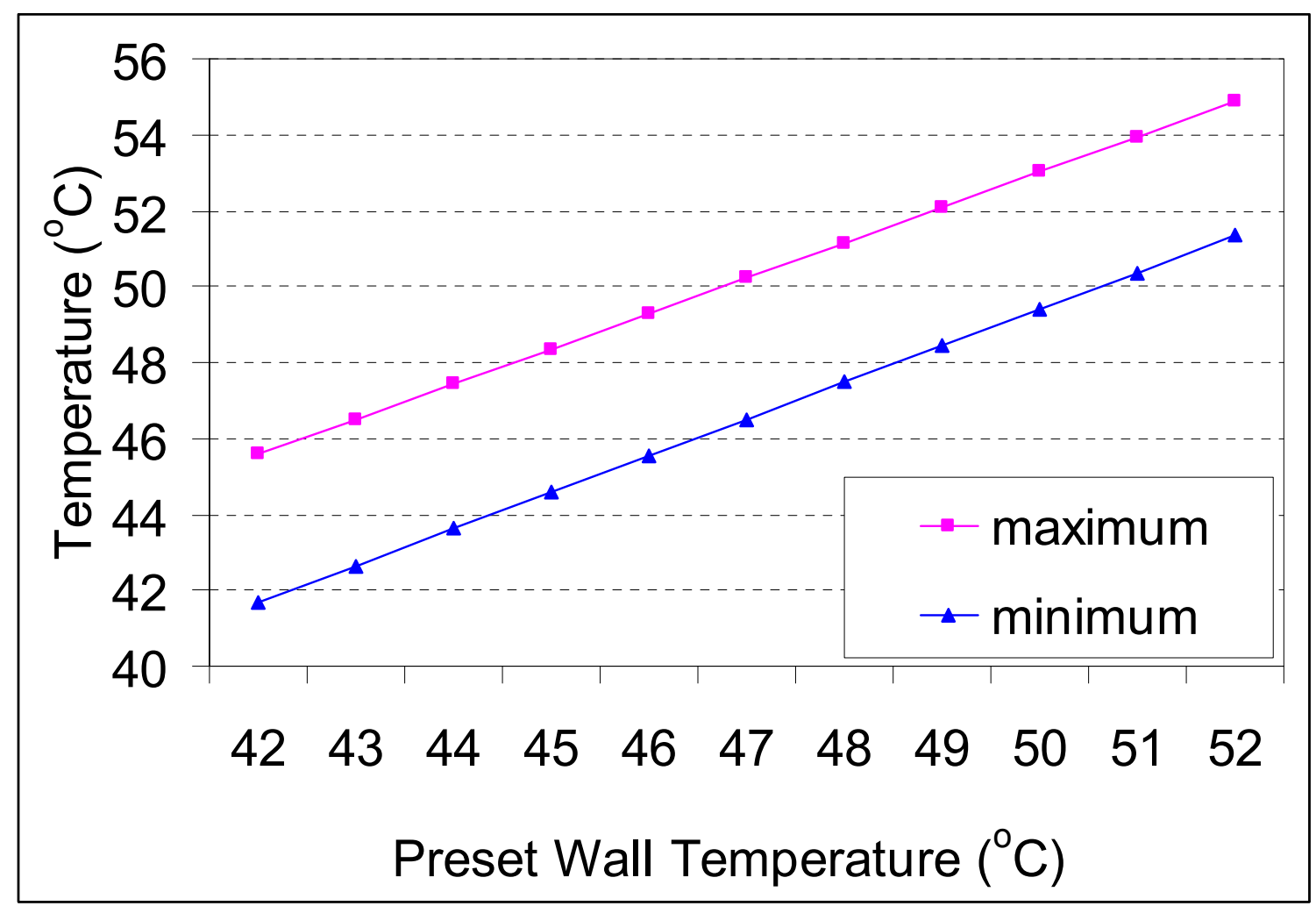

Figure 45 Maximum and minimum filter face temperatures with different preset secondary wall temperatures 


\section{Captured PM Mass}

At very low PM emission levels, PM mass captured on the filter will be very small. Within the constraint of the 2007 standards, an optimization goal for the PM measurement system mass is to find a system configuration which can capture as much PM mass as possible. Overall dilution ratio, filter capture efficiency, and the secondary flow rate determine how much PM mass will be captured if diffusive particle losses are ignored. The secondary flow rate also determines the filter face velocity which affects filter capture efficiency. Figure 46 shows modeling results of cumulative PM mass captured on the filter with different primary flow rates for an FTP run. Figure 47 shows modeling results of total PM captured with different primary flow rates, secondary flow rates and secondary dilution ratios. Estimated real time PM mass emissions from the 2004 Cummins ISM370 engine, in unit of $\mathrm{g} / \mathrm{sec}$, were applied in the simulations.

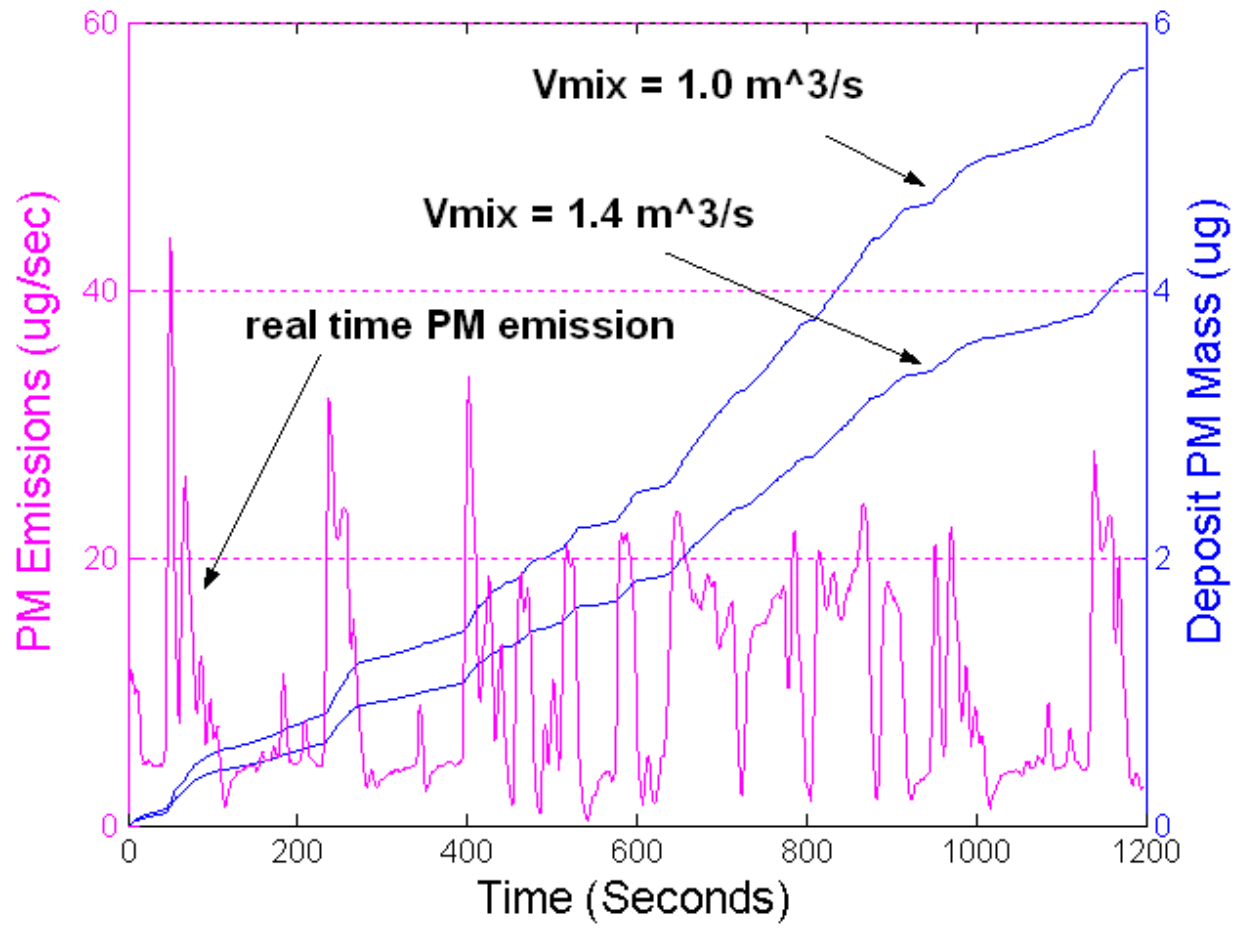

Figure 46 Real time PM emissions and deposited PM mass on the filter with 2 different CVS system flow rate settings.

As Figure 46 shows, deposited PM mass grew slowly when the real time PM emissions were low and increased rapidly when the real time PM emissions were high. A lower primary flow rate corresponded to a higher quantity of captured PM mass. 


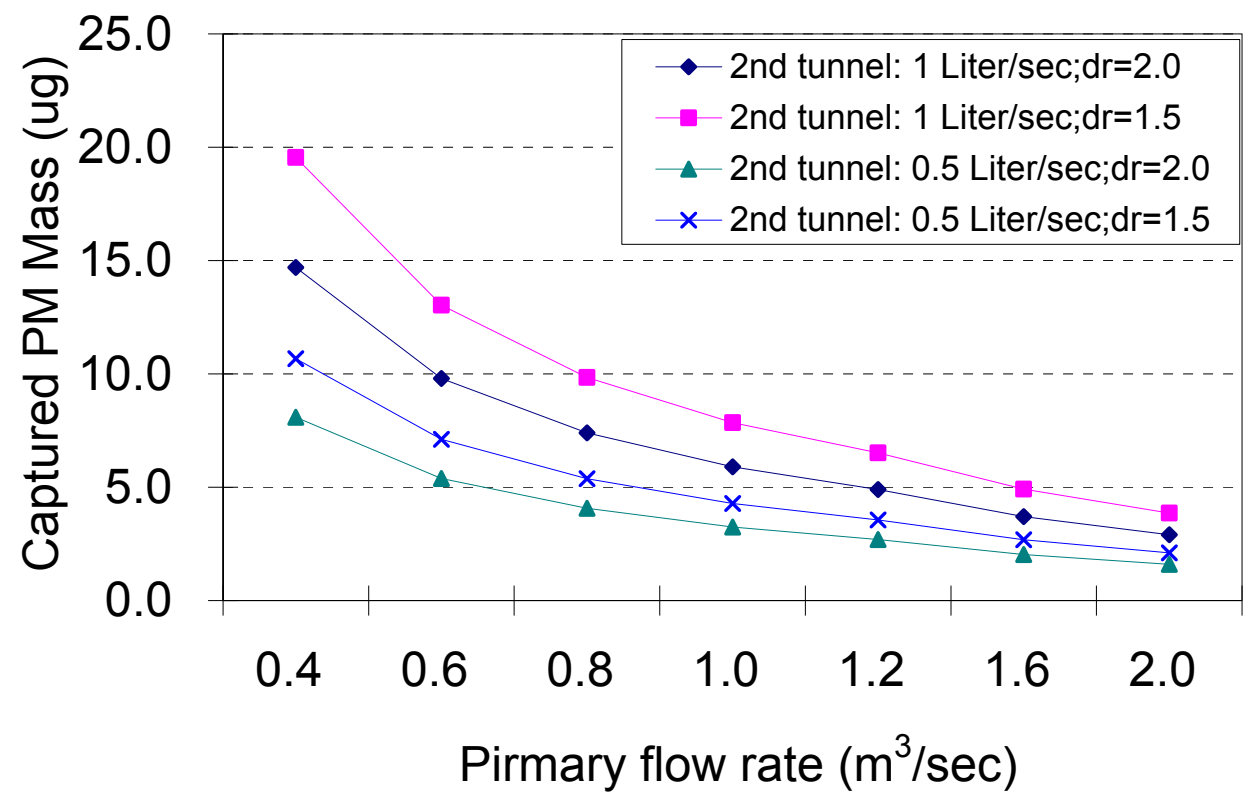

Figure 47 Total PM captured with different primary flow rates, secondary flow rates and secondary dilution ratios for an FTP run

Figure 47 illustrates that the combination of the lowest primary flow rate, the highest secondary flow rate and the smallest secondary dilution ratio resulted in the largest amount of PM mass captured on the filter. High filter face velocity was modeled to reduce the filter capture efficiency. As a result, when the secondary flow rate was reduced by half, total captured PM mass was decreased by about $45 \%$ instead of $50 \%$. A PM measurement system with a primary tunnel of $20.0 \mathrm{~cm}$ in diameter (minimum size requirement by the CFR), a primary flow rate of $0.1 \mathrm{~m}^{3} / \mathrm{sec}$ (minimum value required to maintain a turbulent primary flow), and a secondary flow, minimally diluted, at rate of 1.2 liters per second (filter face velocity $100.00 \mathrm{~cm} / \mathrm{sec}$ ) can capture as much PM mass as possible (141.3 $\mu \mathrm{g}$, not plotted in Figure 46). A secondary tunnel configuration for such a PM measurement to meet the 2007 filter face temperature regulations can always be found as previously discussed (large enough in length and/or small enough in diameter with a wall temperature maintained at a value between $47^{\circ} \mathrm{C} \pm 5^{\circ} \mathrm{C}$ ).

\subsection{Fluent CFD Study}

The parametric study was conducted with the Simulink ${ }^{\circledR}$ model by assuming homogenous properties along the section areas of flows or tunnels. The Simulink ${ }^{\circledR}$ model 
was a one dimensional model for investigating bulk properties, detailed spatial was not examined. Once the dimensional sizes for the primary and secondary tunnels were determined with the parametric study, further understanding of sample flows was obtained using 2D and 3D CFD models. The analysis of CFD modeling helped to determine the location and size of mixing orifice plates, as well as the configurations of the HEPA filter and exhaust pipe housing plenum box.

The commercially available CFD software, Fluent (Canonsburg, PA), was used to investigate flows inside the plenum box and tunnels. The k- $\varepsilon$ viscous model with standard wall functions was used for CFD modeling. This application of $k-\varepsilon$ viscous model for CFD simulation of exhaust plume was proven to be practical in earlier research [117]. The initial CFD study was performed with a simplified 3-D model and compared the results of 2-D model simulations. The initial investigation showed that 2-D axisymmetric models could produce equivalent results for the flows simulated inside the primary tunnel to the results for the 3-D model by setting the appropriate boundary conditions. The 2-D CFD model was applied with constructed grids with much denser meshing than for the 3$\mathrm{D}$ case. To achieve the same accuracy of the 3-D model, the 2-D model requires computational time one to two orders of magnitude lower than the 3-D model would cost. Hence further CFD studies were carried out with 2-D axisymmetric models. Figure 48 shows the 3-D model of plenum box and tunnels with simplified 3-D grids and 2-D grids for one dirty tunnel's exhaust outlet and orifice plate layout.

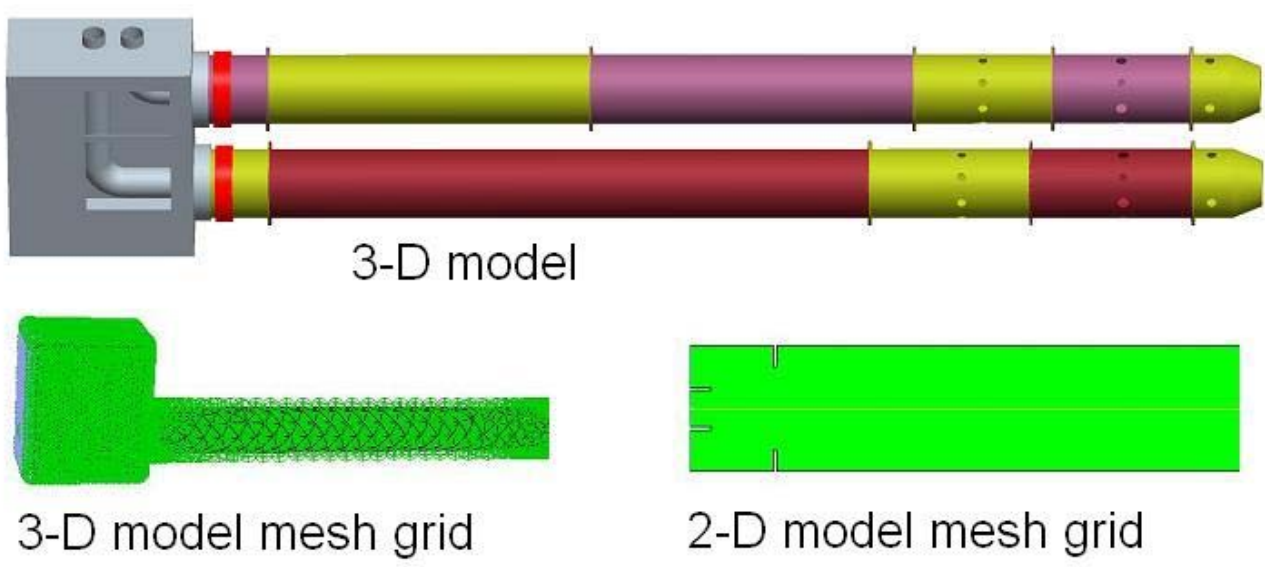

Figure 48 Tunnel model and 3-D/2-D model mesh grids 
The major purpose of this CFD study was to maximize the flow mixing, to minimize the pressure drop across the mixing orifice plate and to minimize exhaust impaction on the orifice plate. CFR part 86 subpart $\mathrm{N}$ and part 1065 requires a Re number of at least 4000 for the primary tunnel to achieve diluted exhaust mixing. However, the researchers sought a higher value of Re to achieve thorough mixing. Also, excessive pressure drop across the orifice would cause deviation from real world exhaust plume dilution conditions. The environmental pressure that the exhaust emits may also affect the evaporation or condensation of certain emission constituents, especially for SOF of PM emissions. The exhaust gas stream impaction on the mixing plate should also be prevented to minimize the particle-surface impaction effects on PM emissions measurement. The objective was achieved by finding the optimal location of exhaust transfer pipe outlet relative to the mixing orifice plate as well as the size of orifice plate. The final position and size chosen had a maximum pressure drop of 6 inches water column across the orifice plate.

The CFD study investigated the positioning of the exhaust transfer pipe outlet distance at 1, 6, and 12 inches from the orifice plate, and mixing orifice diameters of 6,8 , 10 , and 12 inches while supplying the model with different total flow rates and dilution ratios. To demonstrate how the CFD model was implemented and the results analyzed, Figure 49 shows the distributions of $\mathrm{CO}_{2}$ mass percentage fractions inside the primary tunnel for tunnels of three transfer pipe outlet locations.

In Figure 49, exhaust transfer pipe outlet distances of 2.54, 15.24, and $30.48 \mathrm{~cm}$ $(1,6$, and 12 inches) with respect to the 12 inch ID orifice plate were investigated. The tunnel flow rate was set to 2000 SCFM with 700 SCFM exhaust flow rate. This tunnel flow corresponds to a typical setting for characterizing a vehicle with an engine size in the 200 to 300 horsepower ( 150 to $220 \mathrm{~kW}$ ) range. The chemical composition of engine exhaust used for the Simulink ${ }^{\circledR}$ model was also adapted here for species transportation modeling. The homogenous $\mathrm{CO}_{2}$ concentration levels inside the tunnel served as a good indicator of complete flow mixing, which is assumed when less than $0.5 \%$ concentration difference between any two points across the tunnel section was achieved. With the 
orifice plate placed at 1 inch from the exhaust outlet, complete mixing was observed at around 3.5 tunnel diameters downstream of orifice plate. For tunnels where the orifice plate was set at 6 and 12 inches, fully developed flow mixing was observed at 4 and 5.5 tunnel diameters downstream of the orifice plate respectively.

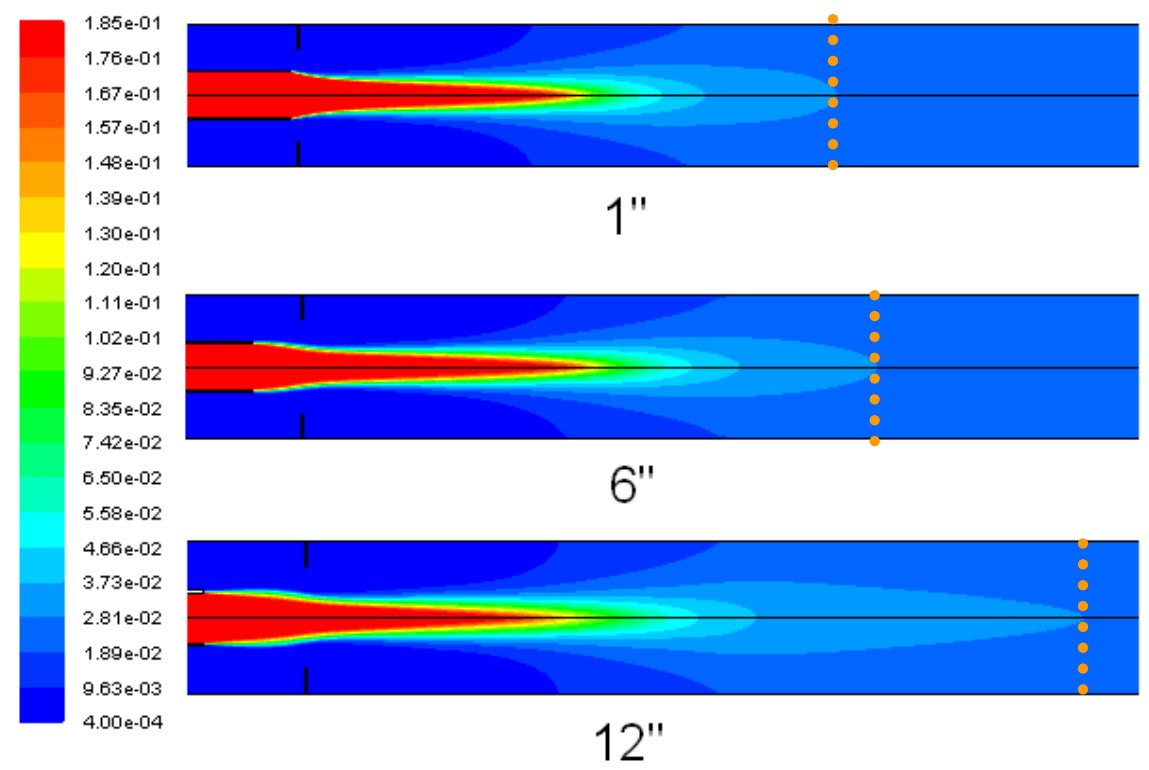

Figure 49 Contours of $\mathrm{CO}_{2}$ mass fractions (\%) for different exhaust outlet locations

As well as looking at the species fractions inside the tunnels, the static pressure, temperature, and particle injection pathlines were also examined. The final plenum box and tunnels were configured to have the exhaust transfer pipe outlets 6 inches from the orifice plates. Four orifice plates ranging from 6 to 12 inches ID square edged, increasing in 2 inch steps, were prepared for changing into the tunnels. The appropriate orifice plate may then be installed for a particular test situation.

The 12 inch ID orifice plate was welded directly to the primary tunnel: the other three plates were designed to be bolted upstream of the 12 inch orifice plate to reduce the orifice size. The cover plated will be bolted to the tunnel which is not used for emissions test. Four orifice sizes were chosen to minimize the pressure drop inside the primary tunnels, while still having flows fully mixed at the sample zone. Table 1 shows an initial 
guide for selecting right size of orifice plate, according to the results of the CFD study and flow rate validation tests using propane injections.

Table 4 Orifice size selection guide

\begin{tabular}{|c|c|}
\hline $\begin{array}{c}\text { CVS Flow } \\
\text { Rate (SCFM) }\end{array}$ & $\begin{array}{c}\text { Orifice Plate } \\
\text { ID (Inches) }\end{array}$ \\
\hline$<600$ & 6 \\
\hline $600 \sim 1200$ & 8 \\
\hline $1200 \sim 1800$ & 10 \\
\hline$>1800$ & 12 \\
\hline
\end{tabular}

\subsection{Gaseous Emissions Measurement System}

The gaseous emissions measurement system was designed to be capable of measuring raw exhaust and diluted emission levels. Emissions levels vary continuously over transient test cycles. Final emissions values must be determined with correction for background level. The background sample flow is taken from a sample probe located immediately after the HEPA filters inside the plenum box. A diluted sample flow is also drawn from the sample probe installed at the primary sample zone, providing a bag of composite diluted exhaust, which is analyzed along with the background bag. Figure 50 shows the plumbing of the gaseous emissions measurement system.

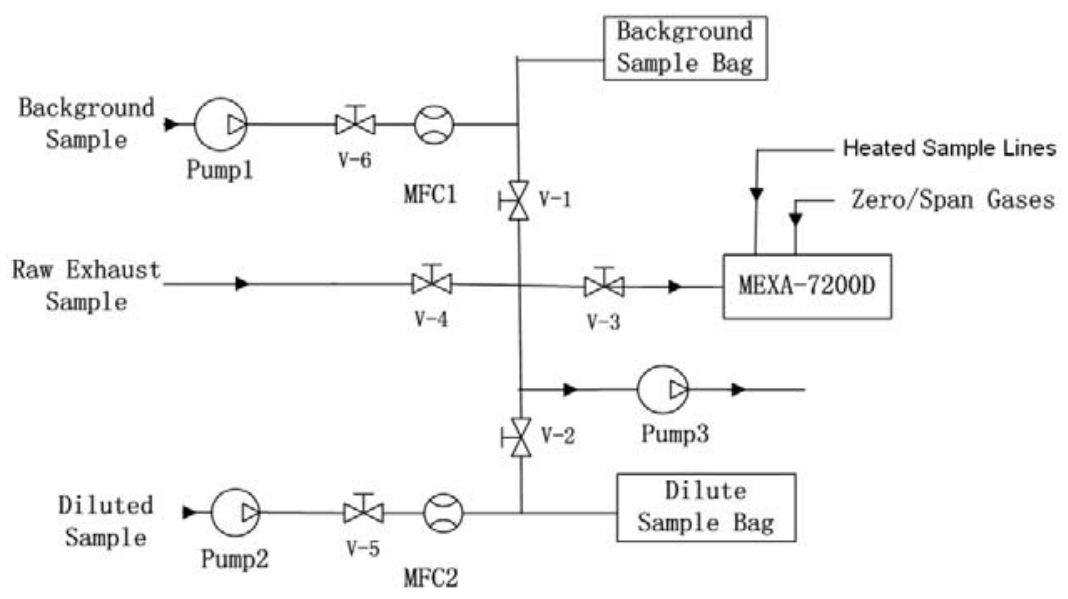

Figure 50 Gaseous emissions measurement system 
While the purpose of the background batch sampling is to correct for background gaseous emission levels, the diluted batch sampling provides for a check on integrated continuous values for quality control purposes. In some cases, where the emissions vary over a wide concentration range over a cycle, a dilute bag analysis may also provide a more accurate assessment of those species than can be obtained by integration. This is often the case for $\mathrm{CO}$ from legacy diesel vehicles over severe transient cycles. Raw exhaust batch sampling for the new system is achieved by switching the raw exhaust sample connection to the diluted sample input line and using appropriate batch sample bags for the raw exhaust.

The Horiba MEXA-7200D motor exhaust gas analyzer (Figure 9) is equipped for the gaseous emissions measurement in the container. At the time of this dissertation, configuration of MEXA-7200D could measure regulated emissions, including THC, CO, $\mathrm{CO}_{2}$, and $\mathrm{NO}_{\mathrm{x}}$. It consisted of eight basic units: the gas divider, the MCU, the IFC, the ANR, the PSU, the SVS, the SHS and the OVN-700 module [137].

The MCU was a PC running the Linux RedHat operating system. Software in the MEXA MCU controled and monitored all other modules of MEXA unit, and could be controlled locally or over an Ethernet link by a host PC. The IFC was a networking device providing communication between all other modules of the MEXA. The ANR could be fitted with up to five 5 analyzer modules. These analyzer modules could be installed and removed from the ANR using basic tools. In the present system, an AIA721A CO analyzer, an AIA-722 CO/ $\mathrm{CO}_{2}$ analyzer and a CLA-720 "cold" $\mathrm{NO}_{\mathrm{x}}$ analyzer were installed on the ANR. The AIA-721A CO analyzer can measure $\mathrm{CO}$ emissions over two preset ranges: 50-2,500ppm and 100-5,000ppm. The AIA-722 $\mathrm{CO} / \mathrm{CO}_{2}$ analyzer measured $\mathrm{CO}$ levels over $0.5-12$ volume percent (vol\%) and $\mathrm{CO}_{2}$ levels over 3-20 vol\%. The SVS controlled the operational and calibration gases that went to the analyzer modules. The SHS filtered, conditioned and pumped the exhaust sample gas to the analyzer modules. The SHS unit was located on a stand alone cart and moved into the bottom section of MEXA-7200D rack. The OVN-700 module combined a FIA-725A THC analyzer and a CLA-720MA $\mathrm{NO}_{\mathrm{x}}$ analyzer as a joint solution for analyzers operated 
under sample-heated conditions. The FIA-725A THC analyzer measured THC emissions over 10-50,000ppm and the CLA-720MA NO analyzer measured $\mathrm{NO}_{\mathrm{x}}$ emissions over 10-10,000ppm [137].

\subsection{PM Sampling System}

Separate from the gaseous emissions measurement part of the transportable laboratory, the PM emissions measurement sub-system was not entirely continaed within the container. The PM filters were pre- and post-test weighed in a class 1000 clean thermal stabilization and weighing enclosure located at the CAFEE at WVU. The onboard part of the PM sampling system is shown schematically in Figure 51. Figure 52 shows the inside view of the actual enclosure.

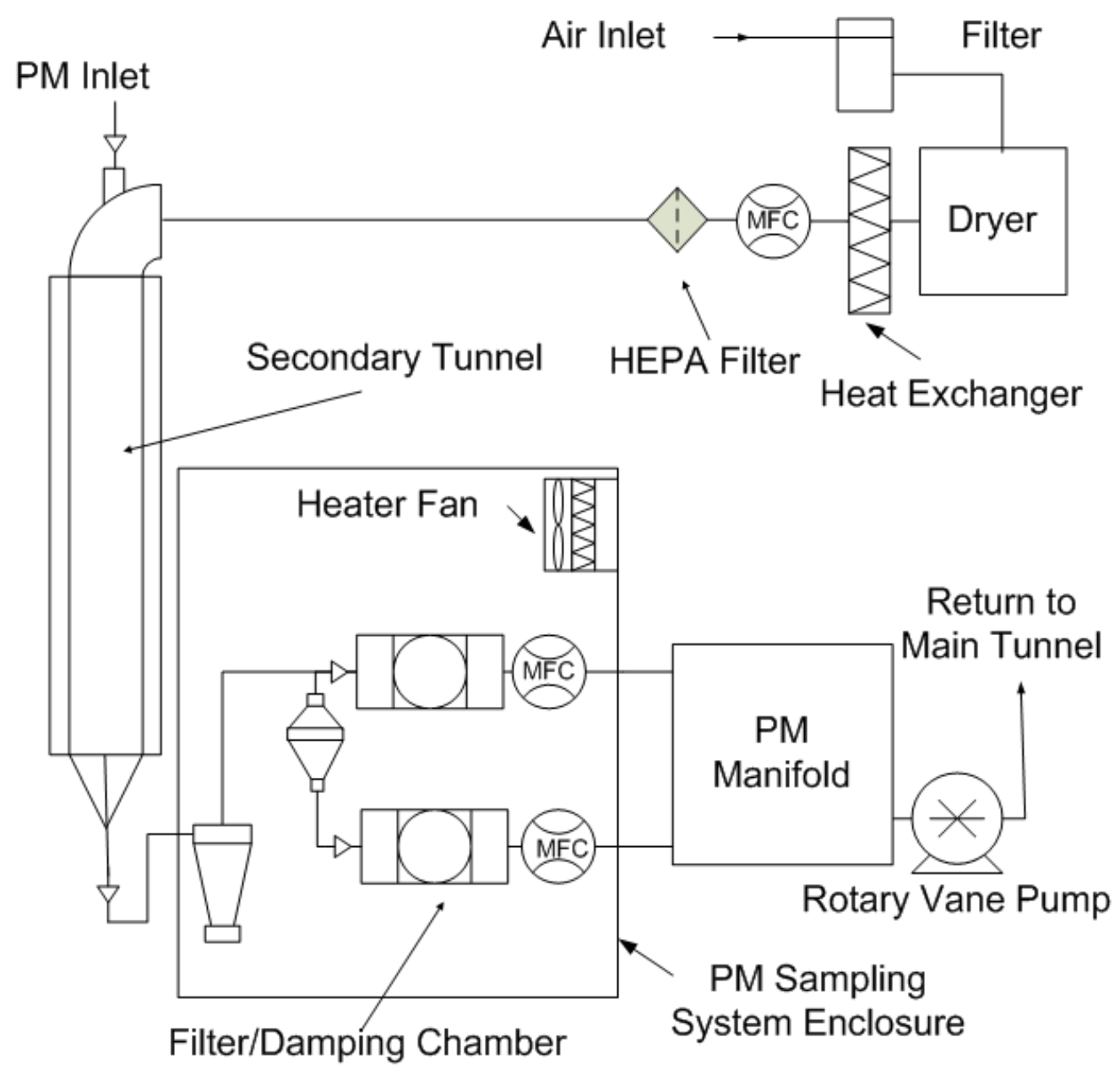

Figure 51 Diagram of PM sampling system 


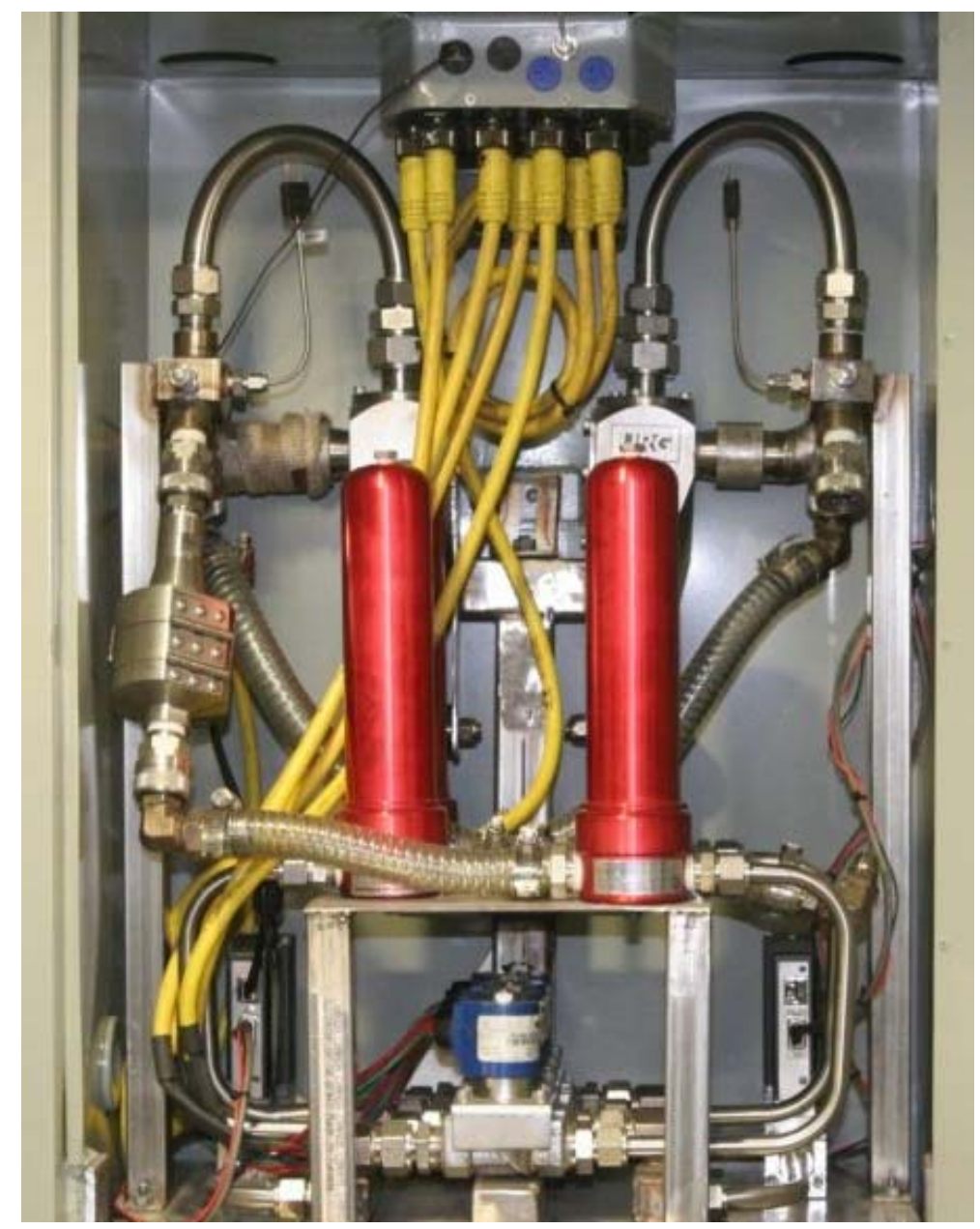

Figure 52 Photo of the PM sampling enclosure

The secondary dilution air passed through a primary filter then was cooled to remove moisture. The dry air was reheated to $25 \pm 5^{\circ} \mathrm{C}$ as required by CFR Part 1065 . The conditioned dilution air was introduced to the PM sample flow drawn from the primary tunnel and flowing through the secondary dilution tunnel. The size of the secondary tunnel shown in Figure 51 was determined by Simulink ${ }^{\circledR}$ modeling. The temperature of the secondary tunnel wall is controlled to be at $47^{\circ} \mathrm{C}$. At the opposite end of the secondary tunnel, the PM sample flow was drawn into an enclosure containing the PM cyclone and PM filter holder. The ambient temperature inside this enclosure was also controlled to $47^{\circ} \mathrm{C}$ in order to maintain the required filter face temperature of $47 \pm 5^{\circ} \mathrm{C}$. The actual enclosure had two sets of PM cyclones and filter holders, although only one is showed in Figure 52. 


\subsection{CVS Flow Control}

The CVS system mass flow rate must be held close to a set point for CFR Part 1065 compliance, although it could be made variable, within constraints, for constant dilution ratio research experiments. If the CVS system fails to maintain the constant flow rate, all cycle-averaged measured gaseous and PM emissions would be invalid because the emissions data reduction computations use the instant CVS mass flow rate over the cycle as one of major input variables. The first transportable emissions measurement laboratory at CAFEE used different sizes of CFV to maintain the CVS flow rate. Such a configuration required significant power consumption to create enough vacuum. It also required flow rate calibration each time a CFV was changed, and suffered from variability of flow in proportion to the square root of absolute temperature, which can imply $5 \%$ variability. The CVS system of the new transportable laboratory minimizes this variability because it was designed based on a SSV. An SSV based CVS system can be calibrated over its whole flow rate range and requires reduced power consumption. Changing the set point of the CVS flow rate over the SSV's measurement range would require no component replacement.

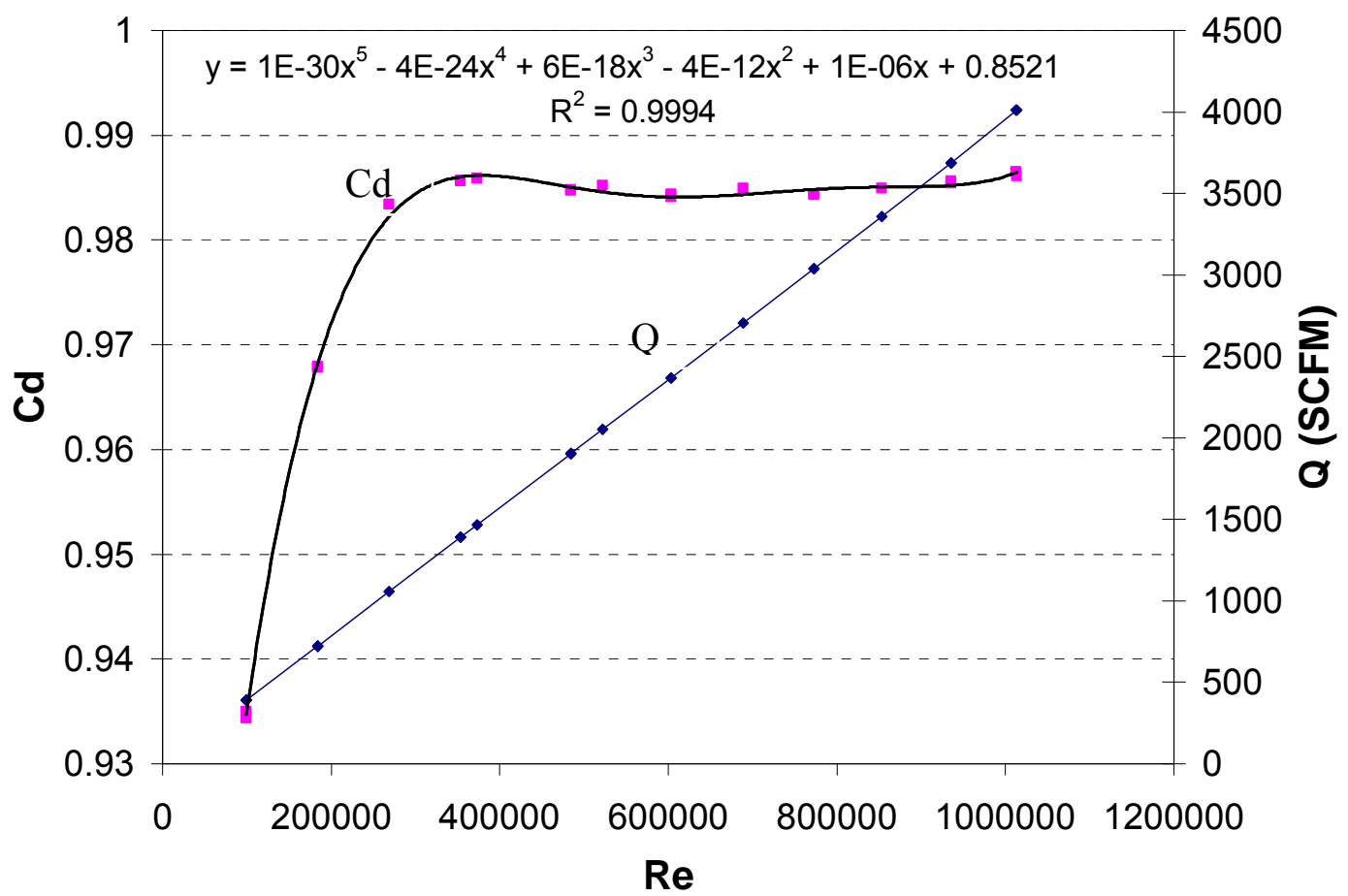

Figure $53 \mathrm{Cd}$ and flow rate vs. Re for the calibrated SSV 
CVS flow was measured continuously by a SSV. Figure 53 shows the coefficient of discharge $(\mathrm{Cd})$ and flow rate plotted against Re for the calibrated SSV. The SSV installed on the transportable laboratory was supplied with 300 series Schedule 5 stainless steel pipe sections, with a nominal internal diameter of 12 " The SSV throat diameter was 6.26 ". To ensure the accuracy and repeatability of SSV flow rate measurement, a straight section of Schedule 5 pipe, ten feet in length, was flanged and attached to each end of the subsonic venturi to minimize the flow wakes, or eddies, or flow circulation which might be induced by pipe bends or coarse inside walls. This particular SSV was calibrated with a reference SSV from 400SCFM to 4000SCFM following the procedure defined in 40 CFR $\$ 1065.340$ [34], and the flow rate of the SSV was calculated, in real time, using the equations in $40 \mathrm{CFR} \S 1065.640$ and $40 \mathrm{CFR} \S 1065.642$ [34]. The key for the SSV calibration was to find a polynomial equation to fit $\mathrm{Cd}$ as a function of $\mathrm{Re}$.

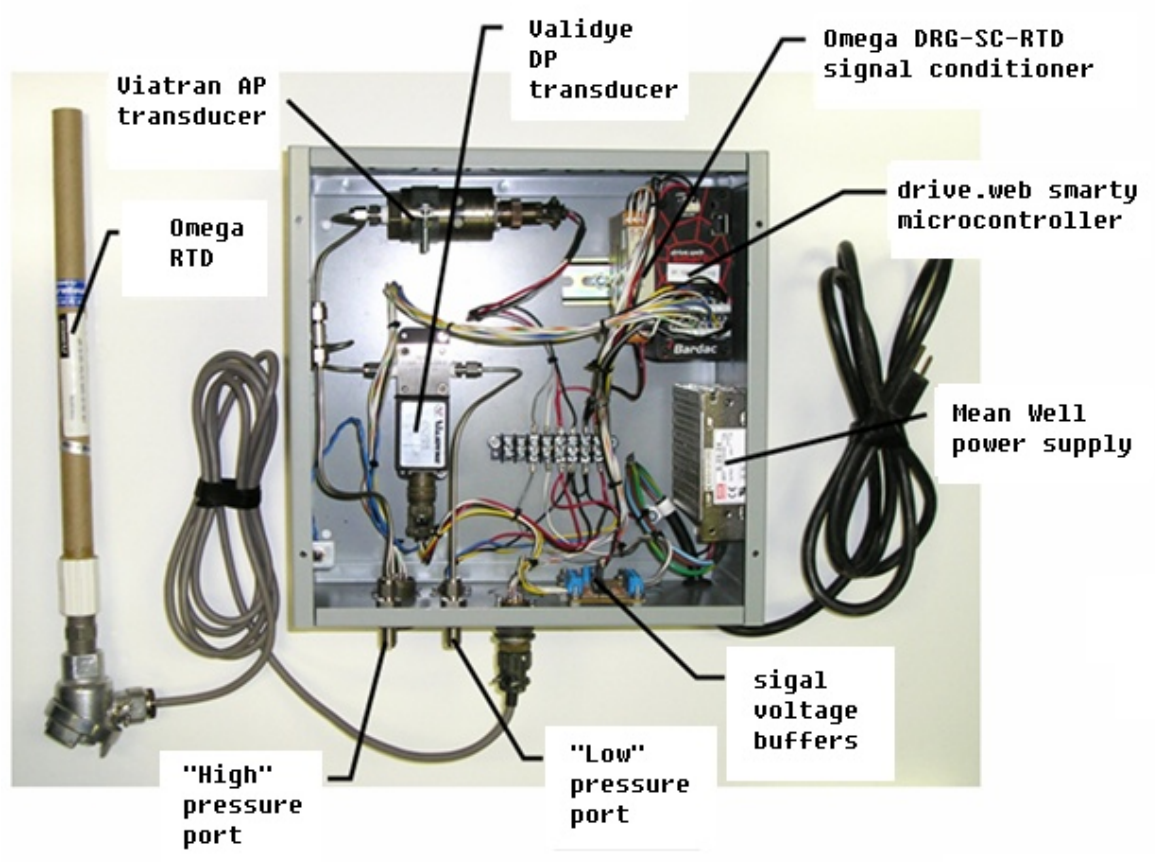

Figure 54 View of the complete flow rate control unit

A Cincinnati Fan RBE-19 blower with a direct drive configuration was selected. The flow rates measured by the SSV were calculated with the calibration equations real time by measuring the SSV's throat differential pressure, and the upstream pressure and 
temperature. The accuracy and repeatability of the temperature and pressure transducers were of prime importance when complying with the requirement for a no-greater-than $2 \%$ error in the controlled flow rate. However, an unavoidable consequence of such tight specifications is the paucity of commercially available devices from which to choose. Ultimately, the following transducers were procured: a Validyne P855 differential pressure (DP) transducer with a maximum response of 50 inches of water $(12.5 \mathrm{kPa})$; a Viatran DC245 15psi (103.4kPa) absolute pressure (AP) transducer; and an Omega Engineering, Inc, PR-14-2-100-3/16-E-AP air-tip A-grade European curve platinum RTD. In the light of the relative complexity of the flow rate formula, including the necessity of both calculating the flow rate and adjusting the blower speed in real time, a drive.web smarty $^{\mathrm{TM}}$ _DIN-rail mountable microcontroller unit was obtained from Bardac Corporation. The CVS flow rate control unit is shown in Figure 54.

A proportional-integral-derivative (PID) controller loop was implemented in the smarty ${ }^{\mathrm{TM}}$ controller. The venturi input parameters, including the DP, AP, and venturi inlet temperature RTD, were "normalized" and fed into a recursive algorithm. Here, the actual flow rate was calculated and compared with the desired flow rate in a main PID controller block. The output from this block was converted to a voltage that represents the speed that the blower was required to achieve. This voltage was fed to a Bardac Corporation E-Drive unit, configured to run in "speed mode," where the voltage sent to it sets the speed of the fan unit according to the relationship $10 \mathrm{~V}$ equates to $100 \%$. The input transducer voltages were each filtered to reduce the effects of electromagnetic interference (EMI) on the system. Thereafter, range checking was performed on the RTD and AP sensor signals. The RTD signal conditioner was configured to give an elevated out-of-range voltage signal out if it detects an open or short circuit in the RTD itself. The AP voltage must remain in a narrow range at or below barometric. If any fault was detected, the E-Drive output signal was immediately set to $0 \mathrm{~V}$ and the "enable line" brought low, turning off the unit. All the internal voltage signals were buffered and brought to the main DAQ system. Also, when the difference between the desired and actual flow rate values in the main PID were within $2 \%$ of each other, a "flow stable" signal was sent to the controlling host computer for operator information and recording. 


\subsection{Chassis Dynamometer}

In order to determine the brake-specific emissions over a duty-cycle, an engine dynamometer would be required. It is applicable to use a stationary dynamometer engine test cell with this transportable laboratory. However, more commonly, a vehicle is set on a chassis dynamometer to obtain the brake-specific power consumption from the engine while the vehicle is being tested under realistic driving conditions. A DynLocIV digital dynamometer Controller was located at the DAQ rack and integrated with test system software to control the operation of the chassis dynamometers of CAFEE at WVU. These chassis dynamometers and their activities have been described in numerous prior papers $[146,147,164,165,166,167$, and 168]. CAFEE currently has three chassis dynamometers capable of simulating vehicle operations from 4,000lbs to about $70,000 \mathrm{lbs}$, with the upper limit dependent on vehicle tire diameter. Figure 55 shows the configuration of one heavy duty chassis dynamometer at CAFEE.

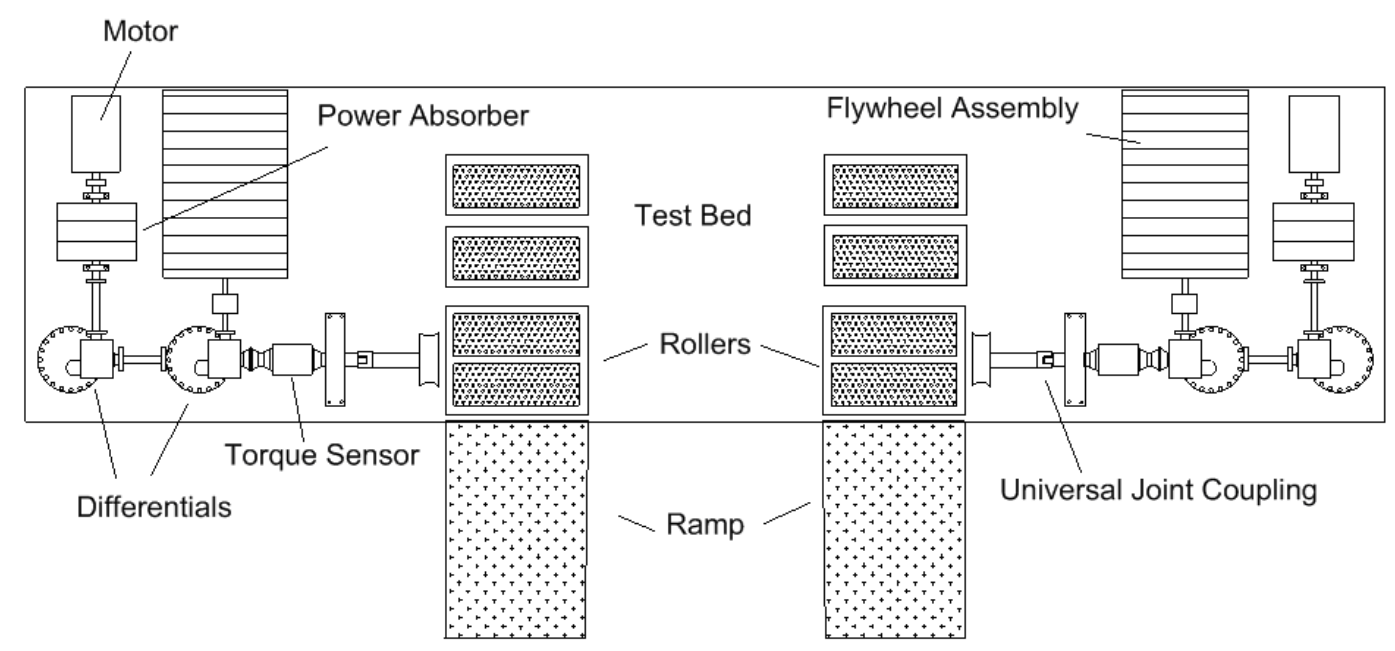

Figure 55 Layout of chassis dynamometer

The chassis dynamometers were constructed as trailers so that they could travel with the transportable laboratory to remote fleet site locations. For the heavy-duty units the power output from the rear (or forward rear) drive axle of the vehicle was transferred to flywheels (inertia simulation), and power absorbers (road load simulation) and AC variable speed motors (light grade motoring simulation) through the drive train units from hub adapters connected to the outboard wheels. Vehicle wheels ran on idler rollers which 
also insured that the left and right wheel speeds were the same, by negating axle differential action. The medium-duty dynamometer employed conventional rollers which receive the power from the wheels.

The details of designs and construction of chassis dynamometers, determination of the power consumption by the dynamometer, and dynamometer calibrations, and have been addressed in previous publications [146, 164, and 165].

\subsection{Structure, Vibration and Noise}

Component structures fabricated in the CAFEE workshop used for the transportable laboratory container were all specified to meet the material strength criteria with safety factors being greater than 5 with only the static stress analyis was performed. Under most circumstances, hand statics calculations were sufficient for the purpose of components' design and construction. However, for some critical supporting frames, Finite Element Analysis (FEA) was also performed to double check and to reinforce the hand-calculated designs. One example of such a component was the tunnel support frame. FEA strength analysis was carried out using the software package "Pro/Mechanic" for the tunnel support structures. Optimal structures were designed for the limited available space in the 30 -foot container. Figure 56 shows one example of strength analysis when applying about 10 times of actual tunnel weight on the support frame.
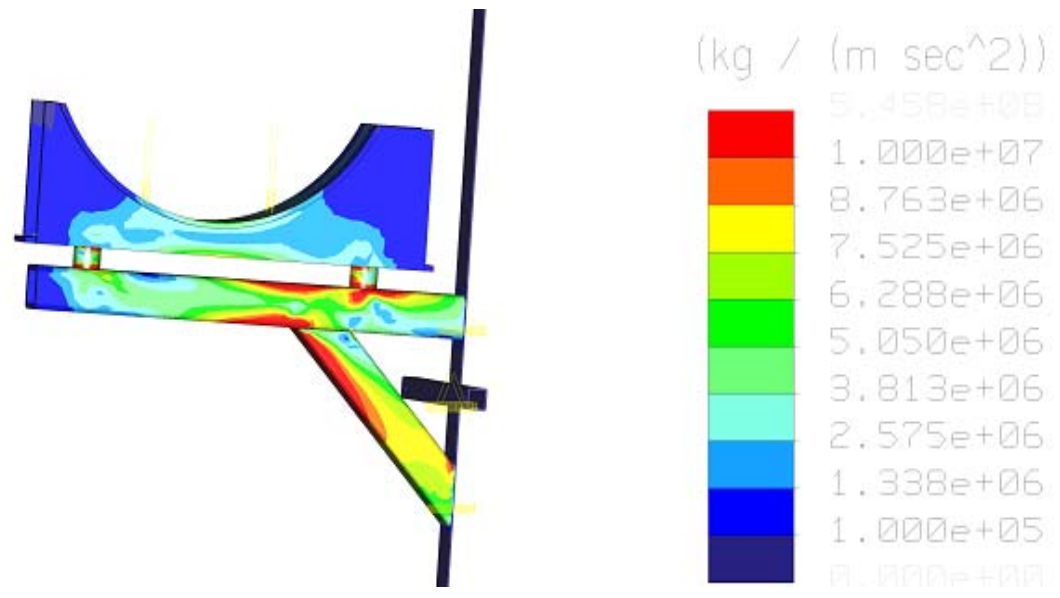

Figure 56 Von Mises stress of the tunnel support 
Vibration, impaction, and noise reduction were also considered during the design and construction stages of structures and equipment. Noise was greatly reduced by constructing a separate operation enclosure for the air compressor and vacuum pumps and setting them on vibration damper mounts. The stainless steel compressed air lines were installed with plastic mounting bases to avoid direct contact with other solid bodies. The tunnel support seats were connected to supporting frames with vibration-damping sandwich mounts. Appropriate commercially available vibration mounts were selected. Simplified analysis for selecting the correct mounts was conducted by simulating the free fall of the load on a linear spring-damper at a speed of $1 \mathrm{~m} / \mathrm{s}$. Behavior of the falling mass was analyzed for different spring rates and damper ratings. The results showed that mounts with higher spring rates and damper rating would reduce vibration more quickly, but this risks higher impaction forces. Figure 57 shows the impaction forces on the top of 6 different mounts by applying an initial downward velocity of $1 \mathrm{~m} / \mathrm{s}$ with $50 \mathrm{~kg}$ mass load. The spring rates and damper ratings decrease from mount 1 to mount 6 . The final vibration mounts selected were combined considerations of vibration responses, impaction ratings, and available mounting positions.

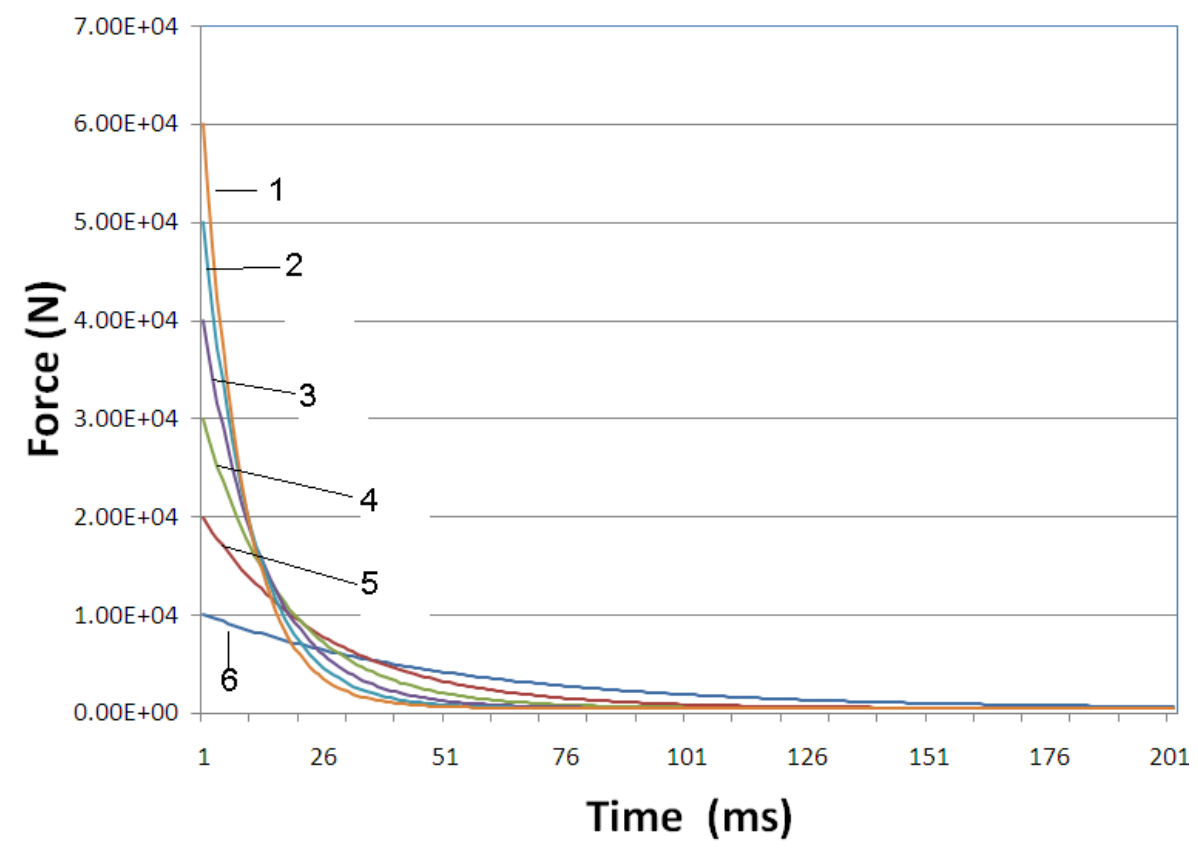

Figure 57 Force impactions on vibration mounts 


\subsection{System Verification}

The CVS system's flow rates were verified with propane injections as described in 40 CFR $§ 1065.341$ [34]. The deviation of SSV measured CVS flow rates was within $\pm 2 \%$ of the mean flow rate values calculated from retrieved propane concentration levels for all applicable CVS flow rate settings with configurations conforming to Table 4.

The verification of MEXA-7200D analyzer and PM sampling subsystem was conducted. The instrumentation part of MEXA-7200D was calibrated and verified by performing the analyzer audit procedures defined in CFR Part 1065 subpart D [34]. Instrumentation verifications done for the MEXA-7200D included linearity, system response, and updating - recording with / without humidified sample, $\mathrm{H}_{2} \mathrm{O}$ interference for $\mathrm{CO}$ and $\mathrm{CO}_{2}$ analyzers, $\mathrm{CO}_{2}$ interference for $\mathrm{CO}$ analyzers, $\mathrm{O}_{2}$ interference for FID analyzer, $\mathrm{CO}_{2}$ and $\mathrm{H}_{2} \mathrm{O}$ quench for CLD analyzer and $\mathrm{NO}_{2}$ to $\mathrm{NO}$ converter efficiency. To illustrate the analyzer audit procedures, Figure 58 shows the collected data for the THC analyzer linearity verification. The data from all analyzers were processed to generate statistics defined in 40 CFR $\$ 1065.602$ [34] and the results passed the linearity standard described in Table 1 of 40 CFR $§ 1065.307$ [34].

\begin{tabular}{|c|c|c|c|c|c|c|}
\hline & Curr & Data & & Curr & it Cur & \\
\hline $\begin{array}{c}\text { Point } \\
\#\end{array}$ & $\begin{array}{l}\text { Z/S Ad justed } \\
\text { Counts }\end{array}$ & $\begin{array}{l}\text { Gen. conc. } \\
\text { (ppmC) }\end{array}$ & $\begin{array}{l}\text { Cut } \\
(\mathscr{x})\end{array}$ & $\begin{array}{c}\text { Meas, conc. } \\
\text { (ppmC) }\end{array}$ & $\begin{array}{c}\text { Error } \\
(g)\end{array}$ & Result \\
\hline 1 & 25631 & 59.70 & 100.0 & 59.63 & -0.07 & Pass \\
\hline 2 & 23282 & 53.73 & 90.0 & 53.78 & 0.05 & Pass \\
\hline 3 & 20883 & 47.76 & 80.0 & 47.79 & 0.07 & Pass \\
\hline 4 & 18485 & 41.79 & 70.0 & 41.81 & 0.05 & Pass \\
\hline 5 & 16089 & 35.82 & 60.0 & 35.84 & 0.05 & Pass \\
\hline 6 & 13686 & 29.85 & 50.0 & 29.85 & -0.02 & Pass \\
\hline 7 & 11282 & 23.88 & 40.0 & 23.85 & -0.12 & Pass \\
\hline 8 & 8893 & 17.91 & 30.0 & 17.89 & -0.10 & Pass \\
\hline 9 & 6499 & 11.94 & 20.0 & 11.92 & -0.13 & Pass \\
\hline 10 & 4102 & 5.97 & 10.0 & 5.95 & -0.41 & Pass \\
\hline 11 & 1734 & 0.00 & 0.0 & 0.04 & 0.04 & Pass \\
\hline
\end{tabular}

Figure 58 Example of THC analyzer linearization data 
The performance of the MEXA-7200D was compared to analyzers of one of existing emissions measurement systems at CAFEE. Results of analyzer comparison for 28 FTP tests using a Cummins ISX565 engine in a transient engine test cell at CAFEE are shown in Figure 59. Tests numbered 1, 8, 14, 20, and 27 were cold start FTP tests (with high spikes of $\mathrm{NO}_{\mathrm{x}}$ and $\mathrm{CO}_{2}$ emissions) and the others were hot start FTP tests. These results show that the MEXA-7200D was able to achieve measurement results in close agreement with the established measurement system at CAFEE, results from which have been reported in numerous prior studies [143, 169, and 170].

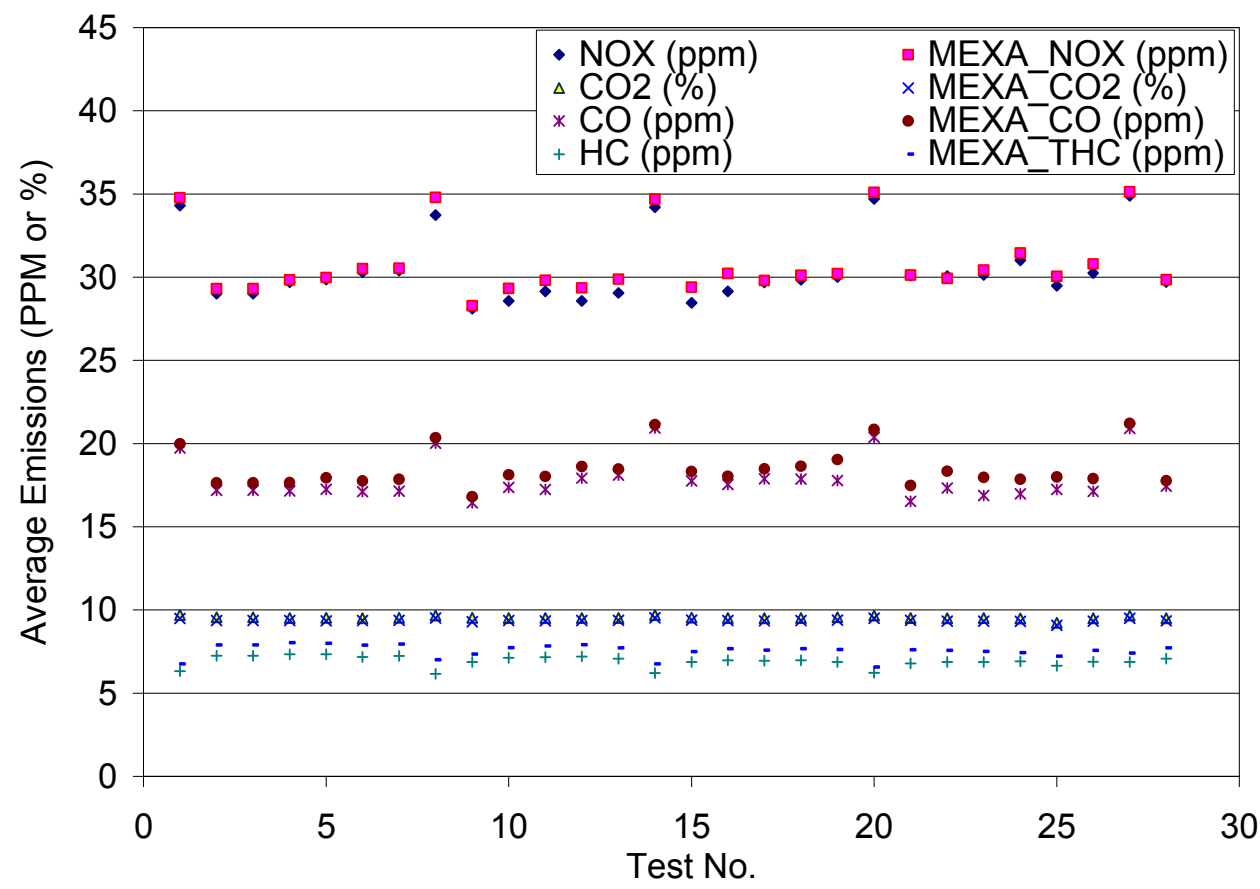

Figure 59 Averaged gaseous emissions of FTP tests

A secondary PM sampling system identical to that in the container was also employed in the transient engine test cell to verify the design and function of the system. The sample flow rates of the secondary tunnel and dilution air flow, the temperature of the dilution air, the temperature of the secondary tunnel wall, the temperature of PM sampling enclosure, and the filter face temperature were monitored during tests. The PM system was proved to be reliable and met CFR Part 1065 specifications. As one of the most important parameters monitored during a test, the filter face temperatures were maintained at $47 \pm 5^{\circ} \mathrm{C}$ for different engines tested and different dilution settings. PM 
emissions sampling was also conducted to evaluate the capability of this system for real PM measurements. Figure 60 shows the measured PM mass emissions in $\mathrm{mg} / \mathrm{bhp}-\mathrm{hr}$ from the Cummins ISX565 engine for 50 hot start FTP tests using this PM emission measurement system.

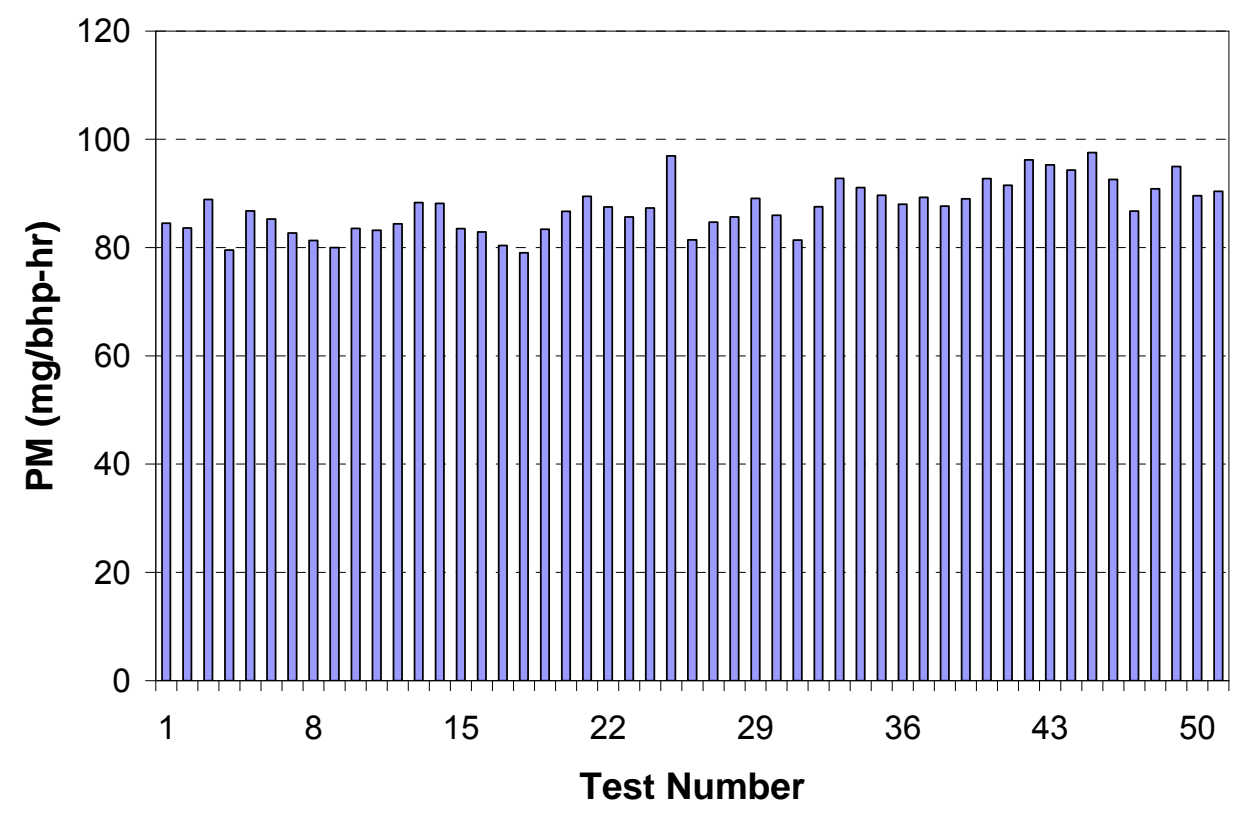

Figure 60 PM emissions of 50 FTP tests (Cummins)

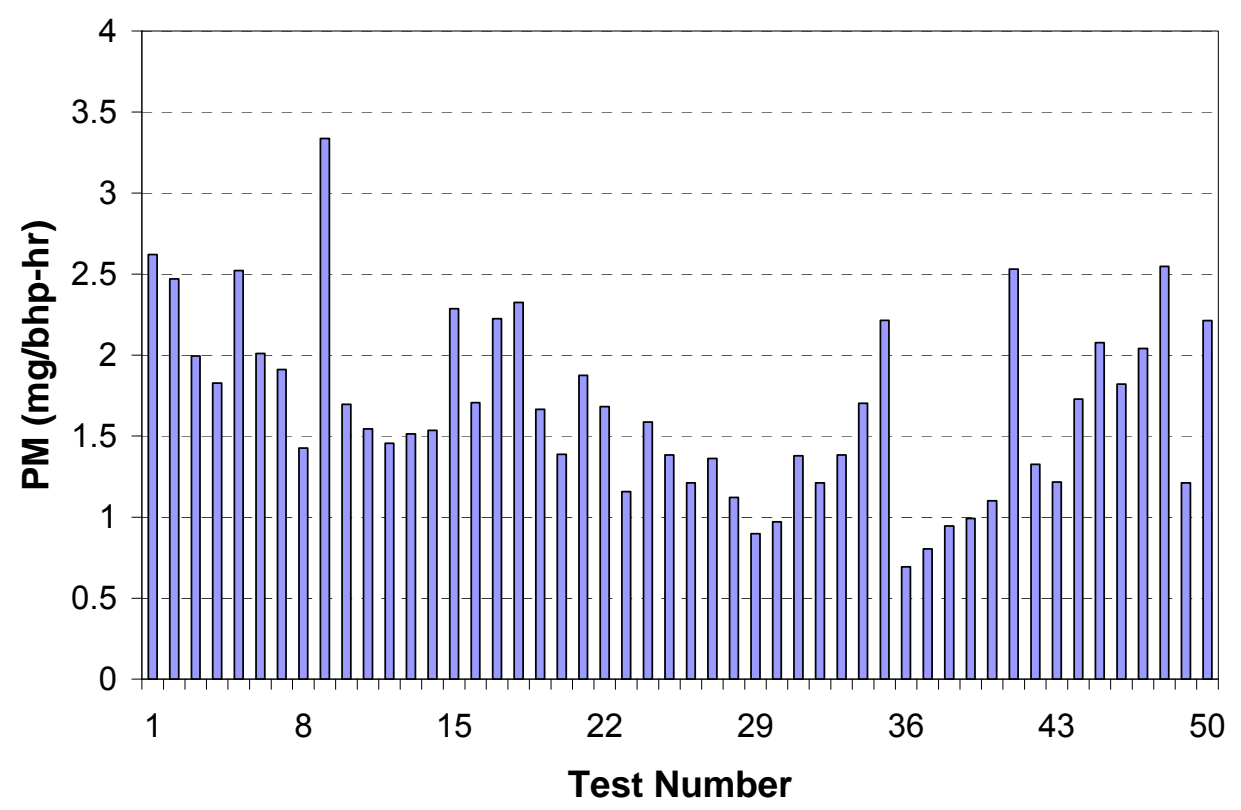

Figure 61 PM emissions of 50 NRTC tests (John Deere) 
The PM emission levels measured from this Cummins engine, which complied to 2004 standards, were about 3 times higher than the 2007 standard, which implies that an exhaust PM filter (trap) must be used on the HDDEs of model year 2007 and later in U.S. This secondary PM measurement system was also used to quantify PM emissions from a 350hp John Deere engine. Figure 61 shows the results of measured PM levels for 50 hot Non-Road Transient Cycle (NRTC) tests from this John Deere engine. This engine was equipped with a Johnson-Matthey Catalyzed Continuous Regenerative Trap (CCRT) to obtain PM levels at or below Tier 4 standards to provide an exhaust stream which would challenge the secondary PM system with very low PM concentrations. It was therefore expected that the trap-equipped John Deere engine would show more variation in percentage than the PM emissions from the Cummins Engine. The literature abounds with information relating to the difficulty of quantifying ultra-low PM mass emissions accurately [37, 171, and 172]. 


\section{ANALYSIS AND EXPERIMENTAL RESULTS}

\subsection{Data Reduction and Analysis Methodology}

\subsubsection{Exhaust Flow Rate}

The raw exhaust flow rate was calculated by converting fuel mass consumption rate to volumetric flow rate at standard condition and adding it to the measured intake air flow rate by the LFE. At the standard condition $\left(20^{\circ} \mathrm{C}, 101,325 \mathrm{~Pa}\right)$, the exhaust density was assumed to be same as the air density. This assumption was discussed earlier in parametric study of Chapter 4 and was verified in Table A1. The fuel consumption rates at different steady state engine running conditions were obtained by recording the fuel tank weight differences over a period of time. The converted fuel consumption rate in SCFM volumetric flow rate with analyzed standard deviation is shown in Table 5 for different engine torque output @1800 rpm. The analysis of standard deviation was performed and is shown in detail in chapter 6 .

Table 5 Fuel consumption rate in SCFM volumetric flow rate

\begin{tabular}{|c|c|c|c|c|c|}
\hline $\begin{array}{c}\text { Engine torque } \\
(\mathrm{Nm})\end{array}$ & 0 (idle) & 34 & 68 & 102 & 122 \\
\hline $\begin{array}{c}\text { Flow rate } \\
(\mathrm{SCFM})\end{array}$ & $0.67 \pm 0.05$ & $1.03 \pm 0.04$ & $1.66 \pm 0.03$ & $2.17 \pm 0.04$ & $2.92 \pm 0.03$ \\
\hline
\end{tabular}

The engine intake air flow rate was measured using a Meriam LFE model 50MC2-2 with serial number of 79676-T2. Four physical properties needed to be recorded for the calculation of the flow rate across the LFE. They were the relative humidity of intake air in percentage, the absolute inlet temperature $T$ in Kelvin (K), the absolute inlet pressure $P$ in inches of mercury column (inHg) and the LFE differential pressure $D P$ in inches of water column (inH2O).

A calibration curve equation sheet came with the LFE from Meriam. This equation was generated by performing a dry air flow rate calibration with a National 
Institute of Standards and Technology (NIST) traceable flow meter by Meriam Instrument. The flow rate was standardized to $70{ }^{\circ} \mathrm{F}$ at $29.92 \mathrm{inHg}$ in units of CFM. According to the manufacturer's calibration data sheet, the calibration curve equation for this LFE for intake air flow rate measurement was:

$$
\text { Flow }(C F M)=12.7637 \times D P-0.00582767 \times D P^{2}
$$

\section{Equation 5. 1}

The DP in $\mathrm{inH}_{2} \mathrm{O}$ was reference to the height of water column at $4{ }^{\circ} \mathrm{C}$ and the differential pressure transducer signal was calibrated at a water temperature reference of $20^{\circ} \mathrm{C}$. The water density needed to be corrected with a correction factor:

$$
\text { Correction factor }=\frac{\rho_{\mathrm{H} 2 \mathrm{O} @ 20^{\circ} \mathrm{C}}}{\rho_{\mathrm{H} 2 \mathrm{O} @ 4^{\circ} \mathrm{C}}}=0.998238
$$

Then the calibration equation could be rewritten as:

$$
\operatorname{Flow}(C F M)=12.7412 \times D P-0.00580715 \times D P^{2}
$$

Equation 5. 3

The flow rate given by the calibration curve equation was not the actual volumetric flow rate. In order to obtain the actual flow rate in CFM (ACFM), following formula was used [139]:

$$
\operatorname{Flow}(A C F M)=\left(12.7412 \times D P-0.00580715 \times D P^{2}\right) \times \frac{\mu_{s t d}}{\mu_{f}} \quad \text { Equation } 5.4
$$

where $\mu_{\text {std }}$ was 181.87 in micropoise, and $\mu_{f}$ was the viscosity of air at flowing temperature in micropoise. Because the engine intake air will always be humid instead of dry, a humidity correction factor must be applied to the calculation of $\mu_{f}$. The Sutherland equation with humidity correction was used for calculating wet air viscosity [139]:

$$
\mu_{f}=\left(\frac{14.58 \times(T)^{3 / 2}}{110.4+T}\right) \times\left(\frac{\mu_{\text {wet }}}{\mu_{\text {dry }}}\right)
$$


The value of $\mu_{\text {wet }} / \mu_{\text {dry }}$ can be looked up in a humidity correction factor chart in Meriam's instruction manual. Since the operational condition range was narrow and a humidity correction factor of 1 was applied by looking up the correction factor chart. Finally, the standard engine intake air volumetric flow rate was obtained by [139]:

Flow $(S C F M)=\left(12.7412 \times D P-0.00580715 \times D P^{2}\right) \times \frac{\mu_{\text {std }}}{\mu_{f}} \times\left(\frac{T_{\text {std }}}{T}\right) \times\left(\frac{P}{P_{\text {std }}}\right) \times\left(\frac{\rho_{\text {wet }}}{\rho_{\text {dry }}}\right) \quad$ Equation 5.6

where $T_{\text {std }}$ and $P_{\text {std }}$ were standard temperature (294.26K) and standard pressure (29.92 inHg) for the LFE, respectively. $\rho_{\text {wet }} / \rho_{\text {dry }}$ was the density correction factor for wet air, which was also available as a look up table in Meriam's instruction manual. For real time measurement, interpolated equations for density correction factor were generated as a function of humidity and temperature from the look up table and implemented in the LabVIEW $^{\circledR}$ DAQ software during real time data measurement:

$\frac{\rho_{\text {wet }}}{\rho_{\text {dry }}}=1.011595-0.0001002142857 * \mathrm{H}-0.0001837857143 * \mathrm{~T}-0.0000041267857 *(\mathrm{H}-60) *(\mathrm{~T}-70)$

Equation 5. 7

where $\mathrm{H}$ was the relative humidity in $\%$ and $\mathrm{T}$ was the measured intake air temperature upstream of the LFE throat in ${ }^{\circ} \mathrm{F}$.

The calculation of engine intake air flow rate could either be done during data post processing or during data acquisition stage by the DAQ software. The latter approach was preferred and was adopted to reduce the data reduction time.

\subsubsection{Dilution Ratios}

Dilution ratio of exhaust inside the tunnel or post-tailpipe was one major factor affecting the DPM size distribution. In order to obtain information of dilution ratios, at least one of $\mathrm{NO}_{\mathrm{x}}, \mathrm{CO}_{2}$ and CO's emission levels needed to be measured. Take the $\mathrm{NO}_{\mathrm{x}}$ emission for example, the dilution ratio of exhaust at a point could be determined by: 


$$
\text { Dilution Ratio }(D R)=\frac{N O_{x_{-}} e-N O_{x_{-}} b}{N O_{x_{-}} m-N O_{x_{-}} b}
$$

Equation 5. 8

where $N O_{x_{-} e}$ was the $\mathrm{NO}_{\mathrm{x}}$ emission level of the engine exhaust, $N O_{x_{-}} b$ was the background $\mathrm{NO}_{\mathrm{x}}$ emission level and $\mathrm{NO}_{x_{-}} m$ was $\mathrm{NO}_{\mathrm{x}}$ emission level of measuring point. The $\mathrm{NO}_{\mathrm{x}}$ level was literally under detectable level at background during the test, so above equation would be simplified as:

$$
D R=\frac{N O_{x_{-}} e}{N O_{x_{-}} m}
$$

Equation 5. 9

It was straightforward to directly measure $\mathrm{NO}_{\mathrm{x}}$ emission levels for dilution ratios calculation of the exhaust after the engine tailpipe. However, the $\mathrm{NO}_{x_{-}} e$ emission level when the engine was tested on the primary tunnel was obtained by following equation instead of directly measurement:

$$
N O_{x_{-}} e=N O_{x_{-}} s \times D R_{-} s
$$

Equation 5. 10

where $\mathrm{NO}_{x_{-}} s$ was the $\mathrm{NO}_{\mathrm{x}}$ level at the sample where fully homogenous mixing was developed and the $D R_{-} s$ is the homogeneous dilution ratio inside the primary tunnel, which was calculated by:

$$
D R_{-} s=\frac{Q_{\text {ssv }}}{Q_{e x}}
$$

Equation 5. 11

where the tunnel flow rate $Q_{s s v}$ is the measured volumetric flow rate of the SSV in SCFM and the exhaust flow rate $Q_{e x}$ is the calculated total exhaust flow rate in SCFM. The exhaust flow rate $Q_{e x}$ was calculated based on chemical balance of burnt fuel and the measurement of intake air flow rate. The dilution ratio of the secondary tunnel was obtained with mass flow controller measured flow rates and DMS500 sample flow rate with following equation:

$$
D R_{2 n d}=\frac{Q_{\text {vacuum }}+Q_{d m s}}{Q_{\text {vacuum }}+Q_{d m s}-Q_{\text {air }}}
$$


where $Q_{\text {vacuum }}$ was the diluted exhaust flow rate went to secondary vacuum pump, $Q_{d m s}$ was the DMS500 sample flow rate, those two flow rates added to the total flow rate of the secondary dilution tunnel. $Q_{\text {air }}$ was the secondary dilution air flow rate. The total secondary flow rate minus the secondary dilution air flow rate was the exhaust flow rate drawn from the primary dilution tunnel into the secondary dilution tunnel.

The dilution ratios of the PPMD were calculated in real time by the PPMD software using the total dilution flow rate and sample flow rate. There was no gaseous emissions measurement involved for the PPMD dilution ratio calculation.

\subsubsection{Comparison of Particle Size Distributions}

The PM size distribution data collected by the DMS500 fast particle spectrometer was present as in $\mathrm{dN} / \mathrm{d} \operatorname{logDp} / \mathrm{cc}$, which is the rate of change of particle number over change of logarithm of the particle diameter per cubic millimeter. An example of PM size distribution data is shown in Figure 62.

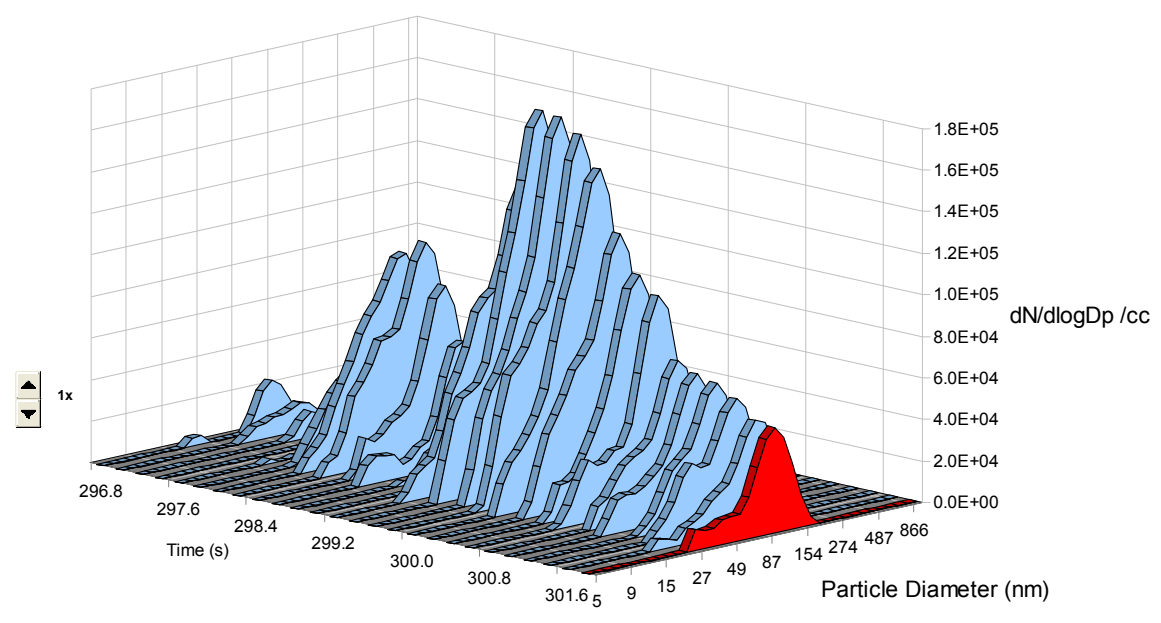

Figure 62 DMS500 dynamic particle spectrum example

The DMS500 data provide size distribution density as $\mathrm{dN} / \mathrm{d} \log \mathrm{Dp} / \mathrm{cc}$ over a given particle diameter size range. The total number of collected particles per cubic millimeter $\left(\mathrm{N}_{\text {total }} / \mathrm{cc}\right)$ could be calculated by numerically integrating the distribution density over the logarithm of particle diameter range, which is actually composed of 38 discrete particle 
diameter sizes, from $4.87 \mathrm{~nm}$ to $1000 \mathrm{~nm}$, with their 10-base logarithm values evenly spaced by 38 divisions of 0.0625 .

The normalized PM size distribution density curve could be obtained by dividing the $\mathrm{Y}$-axis values of the original curve by the total particle number, which was the area under the curve obtained by trapezoidal numerical integration. The unit of Y-axis will become $\left(\mathrm{dN} / \mathrm{DN}_{\text {total }}\right) / \mathrm{d} \log \mathrm{Dp} / \mathrm{cc}$. The normalized curve can also be treated as the continuous probability density function (PDF) curve of the PM size distribution. The Yaxis value would be treated as dimensionless for a PDF curve. Figure 63 shows the PDF curves of 3 PM size distributions. The analytical form of each PDF curve was stored as a look up table with 186 points and the PDF value given a specific point was the interpolated value of a piecewise polynomial cubic spline interpolated from the original curve.

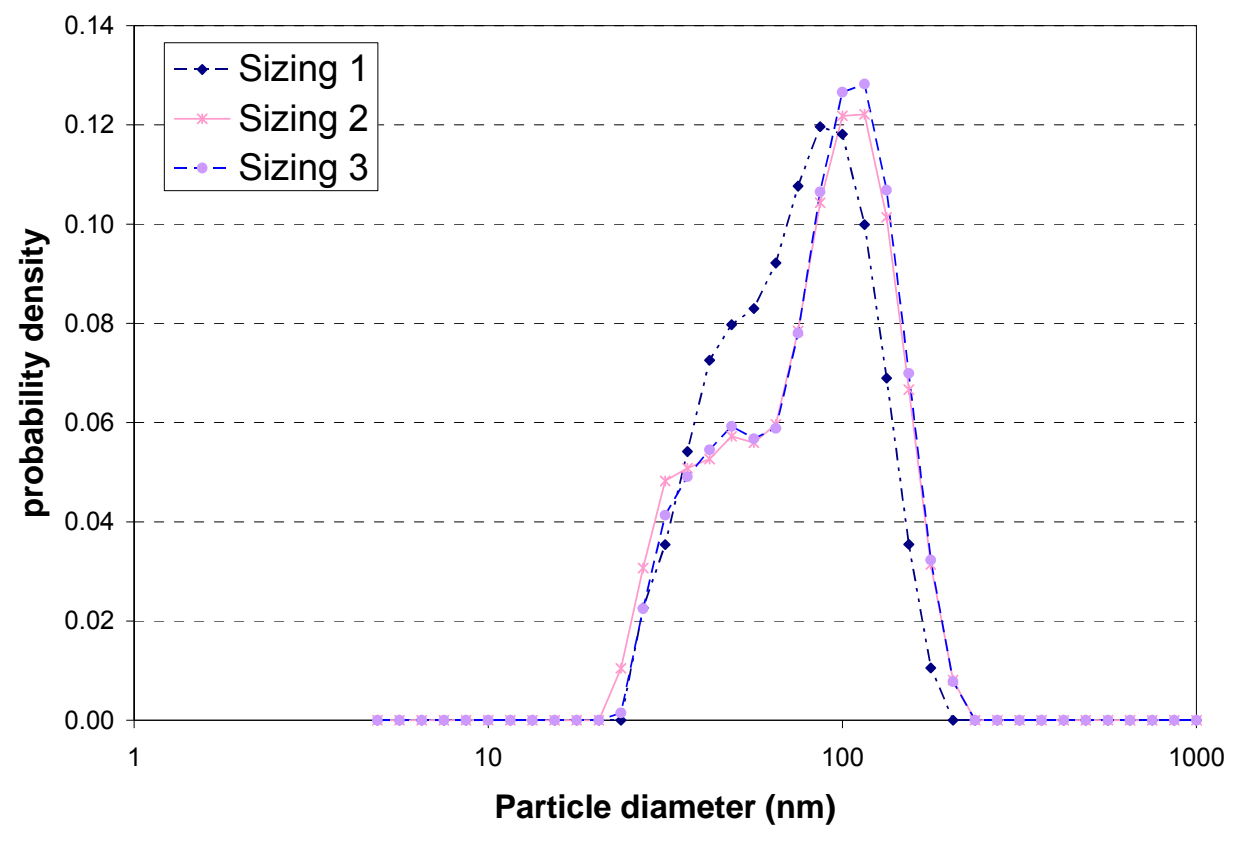

Figure 63 PDFs of 3 PM size distributions

Two different approaches for measuring discrepancy between PM size distributions were developed. The first method was developed based on comparison of two hypothesis test of nonparametric distribution-free test statistics. The second one was based on the concept of least square root. Those methods are shown as: 
a. Kolmogorov Smirnov two-sample test [173] method (Cramér-Von Mises twosample test [173] method compared

b. Least square root method

Both methods were designed to work with normalized PDFs of different PM size distributions and were applied to the size distribution data collected in this research. Only PM size distributions within certain percentage difference for values of total number and /or mass concentration levels were compared to each other. Take threshold of 5\% difference of the total particle number count concentration levels for example (same for mass). The following condition needed to be met before further investigation on the PM size distributions was carried out:

The absolute value of the difference between the total number of collected particles per cubic millimeter $\left(\mathrm{N}_{\text {total }} / \mathrm{cc}\right)$ of PM size distribution $\mathrm{X}$ and that of distribution $\mathrm{Y}$ was less than or equal to 5 percent of the smaller value of $\mathrm{N}_{\text {total }} / \mathrm{cc}$.

$$
\frac{N_{\text {Xtotal }} / C C-N_{\text {Ytotal }} / C C}{\operatorname{Min}\left(N_{\text {Xtotal }} / C C, N_{\text {Ytotal }} / C C\right)} \times 100 \% \leq 5 \%
$$

\section{a. Kolmogorov Smirnov two-sample test method}

The Kolmogorov Smirnov two-sample test is used in the situation where two samples are drawn independently from two possibly different distributions, and the tester wants to find whether the two distributions are identical or not [174]. The test statistic of Kolmogorov Smirnov two-sample test is the greatest vertical distance between the sample empirical cumulative distribution function $S_{1}(x)$ of sample $X_{1}, X_{2}, \ldots, X_{m}$ and the sample empirical cumulative distribution function $S_{2}(y)$ of sample $Y_{1}, Y_{2}, \ldots, Y_{n}$ :

$$
\begin{array}{ll}
T=\operatorname{Max}\left(\left|S_{1}(x)-S_{2}(y)\right|\right) & \left(\text { Two-sided } \mathrm{H}_{1}: f(x) \neq f(y)\right) \\
T_{+}=\operatorname{Max}\left(S_{1}(x)-S_{2}(y)\right) & \text { (One-sided H1: } f(x)>f(y)) \\
T_{-}=\operatorname{Max}\left(S_{2}(y)-S_{1}(x)\right) & \text { (One-sided } \left.\mathrm{H}_{1}: f(x)<f(y)\right) \quad \text { Equation 5. 13 }
\end{array}
$$


If the null hypothesis is true and $m=n$, the exact cumulative distribution function of $T_{+}$ and $T_{-}$is given by [174]:

$$
F(x)=1-\frac{\left(\begin{array}{c}
2 n \\
n
\end{array}\right)}{\left(\begin{array}{c}
2 n \\
n
\end{array}\right)}
$$

where $\mathrm{d}$ is the greatest integer less than $x \cdot n$. For the hypothesis here, it is a two-sided test. The exact two-sided p-value can be found when $m=n[174]$ :

$$
\text { two-sided } p-\text { value }=2 \frac{\left(\begin{array}{c}
2 n \\
n+n t
\end{array}\right)}{\left(\begin{array}{c}
2 n \\
n
\end{array}\right)}
$$

where $t$ is the observed value of the test statistic $T$.

Because the DMS500 gives PM size distribution data is in the form of a density function, it does not provide particle count for any specific particle diameter. Actually, from the strict mathematical point of view, the probability of detecting a particle of given diameter is always zero. In order to perform the Kolmogorov Smirnov 2-sample test, the Rejection-Acceptance algorithm [174] is used to generate random $\mathrm{x}$ values from $\operatorname{PDF} f(x)$ :

(1) Generate a uniformly distributed random number $x$, between $0.6875\left(\log _{10}(4.87)\right)$ and 3, i.e. $x=0.6875+2.3125 r_{1}$, where $r_{1}$ is uniformly distributed between 0 and 1.

(2) Generate a second independent random number $u$ which is uniformly distributed between 0.6875 and the maximum value of size distribution density function, noted as $f_{\max }(x)$, i.e. $u=r_{2} f_{\max }(x)$

(3) If $u<f(x)$, then accept $x$. If not, reject $x$ and go to step (1).

The method of Kolmogorov Smirnov two-sample test can be summarized as two steps. Step one is to generate PM size samples $X_{1}, X_{2}, \ldots, X_{m}$ from $\operatorname{PDF} f(x)$ of distribution $\mathrm{X}$ and $Y_{1}, Y_{2}, \ldots, Y_{n}$ from the PDF $f(y)$ of distribution Y. Step two is to 
perform a Kolmogorov Smirnov two-sample test with null hypothesis $\mathrm{H}_{0}$ and alternative $\mathrm{H}_{1}$ :

$\mathrm{H}_{0}$ : Two samples were generated from the same distribution $\quad f(x)=f(y)$

$\mathrm{H}_{1}$ : Two samples were not generated from the same distribution $f(x) \neq f(y)$

If the null hypothesis is not rejected at the level of significance 0.05 , the conclusion will be made that distribution $\mathrm{X}$ and distribution $\mathrm{Y}$ are not statistically different at significance level 0.05 .

The procedure of Cramér-Von Mises two-sample test method is same as that of Kolmogorov Smirnov two-sample test except the test statistic is changed to:

$$
T=\frac{m n}{(m+n)^{2}}\left\{\sum _ { i = 1 } ^ { n } \left[\left(S_{1}\left(X_{i}\right)-S_{2}\left(X_{i}\right)\right]^{2}+\sum_{j=1}^{n}\left[\left(S_{1}\left(Y_{j}\right)-S_{2}\left(Y_{j}\right)\right]^{2}\right\} \quad \text { Equation } 5.16\right.\right.
$$

The approximate $p$-value for $m+n>17$ can be interpolated from the Cramér-Von Mises quantiles table. The Cramér-Von Mises test technique is expected to use sample data more efficiently than the Kolmogorov Smirnov test method, because its test statistic uses all samples data points. Since the Cramér-Von Mises test is more computationally intensive than the Kolmogorov Smirnov test method and more sensitive to sample noise, the Kolmogorov Smirnov test method was adopted in this research as the non-parametric test statistics for the particle size distribution curves' comparison.

\section{b. Least square root method}

The least square root method does not need to generate random samples. For two normalized PM distribution A and B with PDFs $f_{1}(x)$ and $f_{2}(x)$, this method can also be stated in a way of hypothesis test:

$$
\begin{array}{ll}
\mathrm{H}_{0} \text { : A and } \mathrm{B} \text { are same distribution } & f_{1}(x)=f_{2}(y) \\
\mathrm{H}_{1} \text { : A and B are different distributions } & f_{1}(x) \neq f_{2}(x)
\end{array}
$$


The value of the following integration equation was calculated for the hypothesis test:

$$
\alpha=\sqrt{\int_{0.6875}^{3}\left(f_{1}(x)-f_{2}(x)\right)^{2} d x}
$$

A preset threshold value, for example, 5\% is used to make the decision on two compared distributions will be made based on:

$$
\begin{aligned}
& \alpha>0.05 \text { (Decision: reject } \mathrm{H}_{0}, \mathrm{~A} \text { and } \mathrm{B} \text { are different distributions) } \\
& \alpha<0.05 \quad \text { (Decision: do not reject } \mathrm{H}_{0}, \mathrm{~A} \text { and } \mathrm{B} \text { are identical distributions) }
\end{aligned}
$$

\subsection{Dilution Tunnel Test}

This study investigated the PM evolution inside a full scale primary dilution tunnel as well as at the end of a secondary PM dilution tunnel, just upstream of the PM sample filter. A parametric approach was employed with varying primary and secondary dilution ratios and multiple engine operating conditions. A Cambustion DMS500 Fast Particulate Spectrometer was employed for PM particle sizing.

DPM size distributions or mass concentrations reported in this study were all corrected, according to respective dilution ratios, to exhaust stack equivalent levels. The engine was operated at a speed of $1800 \mathrm{rpm}$ with torque settings of $102 \mathrm{Nm}$ and $122 \mathrm{Nm}$. The following discussion is focused on the results of the engine operating at $102 \mathrm{Nm}$ torque output, but size distributions behaved similarly for both engine operating conditions.

\subsubsection{Primary Tunnel}

The measured dilution ratios across tunnel sections for two primary tunnel flow rate settings are shown in Figure 64. CFD simulation results, along with information from Figure 18 and Figure 64, revealed a clear pattern of flow mixing inside the primary tunnel after the orifice mixing plate. Immediately after the orifice plate, the dilution air created an air sheath to surround the exhaust flow. The mixing first happened at the interface between the dilution air and the exhaust flow. Downstream, the interface between the air 
sheath and the exhaust flow became less distinct. Meanwhile, a backflow circulation zone was established close to the tunnel wall, in an area downstream of the orifice plate and upstream of a location where the flow was fully mixed. The existence of a backflow circulation zone resulted in least diluted exhaust fractions being probable nearer the section centerlines, contrary to intuitive assumptions that this would be most likely near the tunnel wall. Once the flow was fully mixed, the backflow did not occur, and the flow was homogeneously diluted across sections.

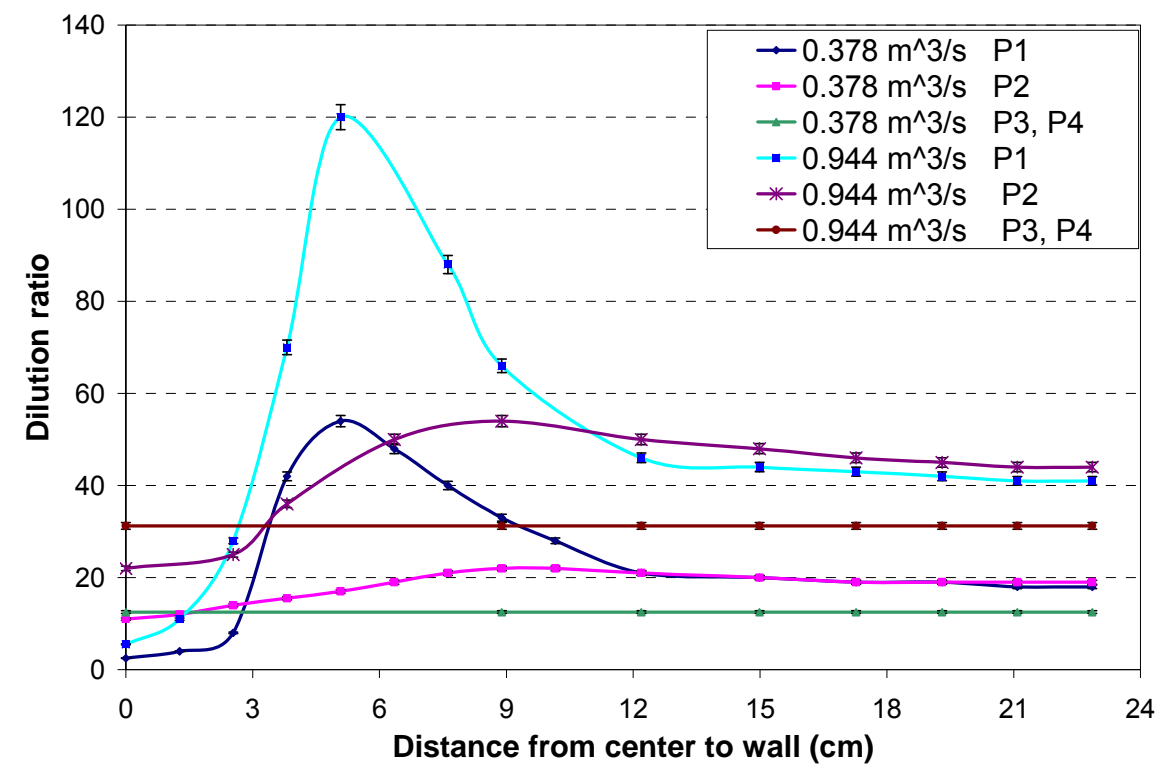

Figure 64 Dilution ratios across tunnel sections

Since the engine was equipped with a DPF, it was expected that a high number of ultrafine $(<100 \mathrm{~nm}$ in diameter) or nanoparticles $(<50 \mathrm{~nm}$ in diameter $)$ would be observed under some dilution conditions. New particle formation is mainly the result of nucleation of volatile material (soluble organic fraction - SOF) in the exhaust, whereas pre-existing particles grow by the processes of adsorption or condensation. These two distinct modes of particle formation and growth are known as nucleation mode and agglomeration mode, respectively. Because most solid carbon mass of raw exhaust PM was removed by the DPF, there would be little surface area available for agglomeration to occur, and so it was expected that the nucleation mode would be the dominant particle process. 
Preliminary analysis of particle size distribution data proved that mainly particles in the nano-size $(<50 \mathrm{~nm}$ in diameter) range were detected during the tests. Particle dynamics were extremely nonlinear for Diesel particles in the nano-size range. Researchers have shown that large changes in nano-particle count may result from small or modest changes in sample treatment variables, such as dilution ratio, temperature, humidity, local partial pressures, and residence time [8, 102, and 175]. The information on dilution ratios obtained from $\mathrm{NO}_{\mathrm{x}}$ concentration levels were not only used to correct the measured particle size distributions to their stack equivalent values, but were also used to provide information of the flow temperature and saturation ratio (ratio of partial pressure to saturated vapor pressure) of the condensable species at the sampling point.

Figure 65 and Figure 66 show particle size distributions as a function of sampling distances from the tunnel centerline at P1 1 foot downstream of the orifice plate. These data were collected at primary tunnel flow rates of $0.378 \mathrm{~m}^{3} / \mathrm{s}$ and $0.944 \mathrm{~m}^{3} / \mathrm{s}$, respectively, and at an engine operating condition of $102 \mathrm{Nm}$ at $1800 \mathrm{rpm}$. Particle size distributions across the $\mathrm{P} 1$ section showed a similar peak shift pattern for both $0.378 \mathrm{~m}^{3} / \mathrm{s}$ and $0.944 \mathrm{~m}^{3} / \mathrm{s}$ primary flow rates.

The size distributions across the P1 section started with a single peak (mean equivalent diameter) at approximately $14 \mathrm{~nm}$ at the center, where the least dilution occurred. As the sample probe was moved toward the tunnel walls, the size distribution peak started shifting toward smaller particle diameters, and a bi-modal (around $7 \mathrm{~nm}$ and $10 \mathrm{~nm}$ ) size distribution was developed before the flow was most diluted, at approximately $5.0 \mathrm{~cm}$ from the tunnel centerline. As the sample probe moved further toward the tunnel wall, the smaller size range peak disappeared, and the size distribution transferred back to a single mode distribution. At the same time, when the sample probe was traversed from the centerline toward the tunnel wall, the total particle count at the sampling point also increased with increasing dilution ratio. The increased total particle count indicated that SOF saturation ratios, at the corresponding dilution ratios, were in favor of particle nucleation. Similar size distributions were also detected at P1 by increasing the engine load to $122 \mathrm{Nm}$ and shown in Figure 67 and Figure 68. 


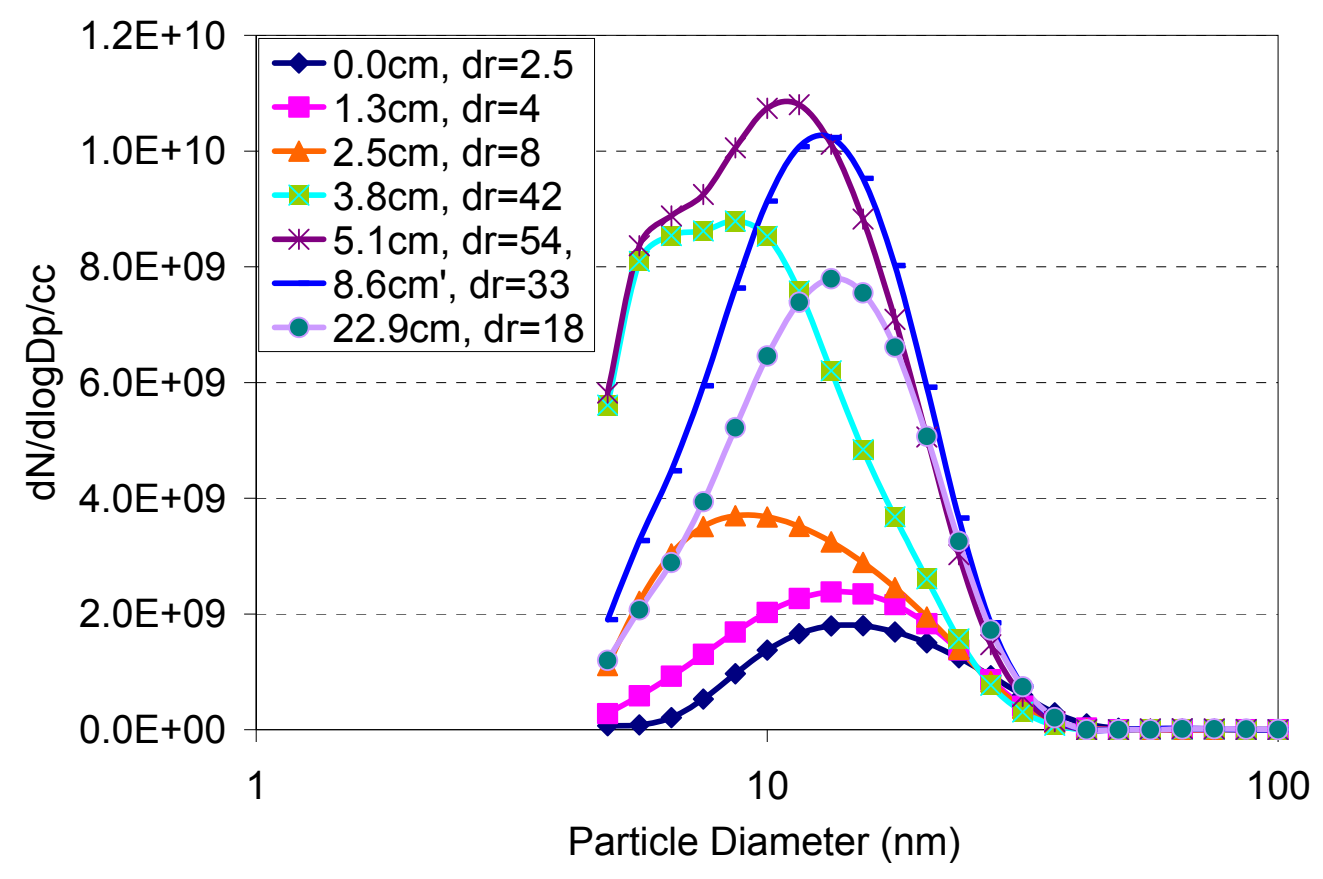

Figure 65 Particle size distributions of the dilution flow at different sampling distance from tunnel cross section center at P1 (primary flow rate $0.378 \mathrm{~m}^{3} / \mathrm{s}, 102$ Nm@1800 rpm)

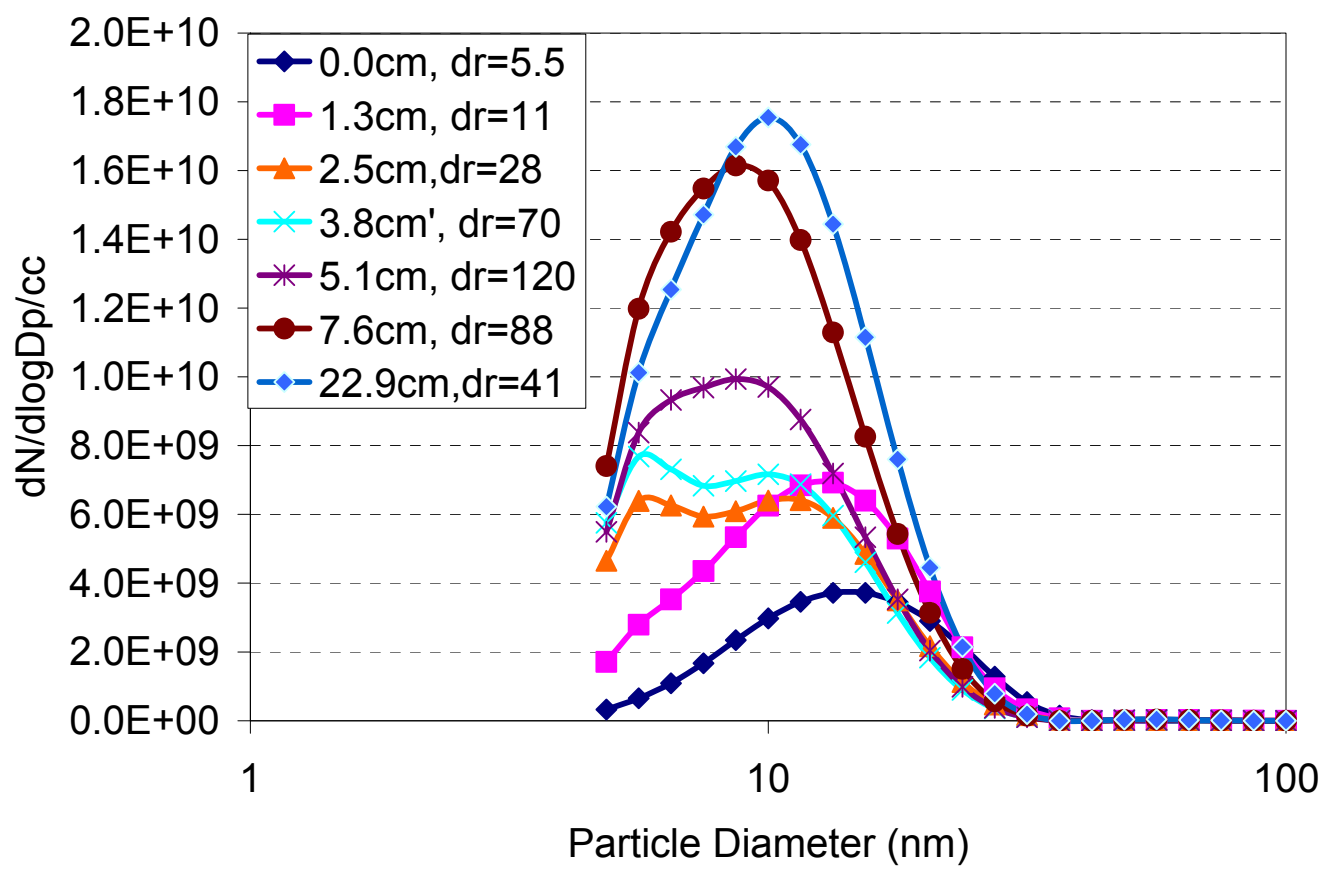

Figure 66 Particle size distributions of the dilution flow at different sampling distance from tunnel cross section center at P1 (primary flow rate $0.944 \mathrm{~m}^{3} / \mathrm{s}, 102$ Nm@1800 rpm 


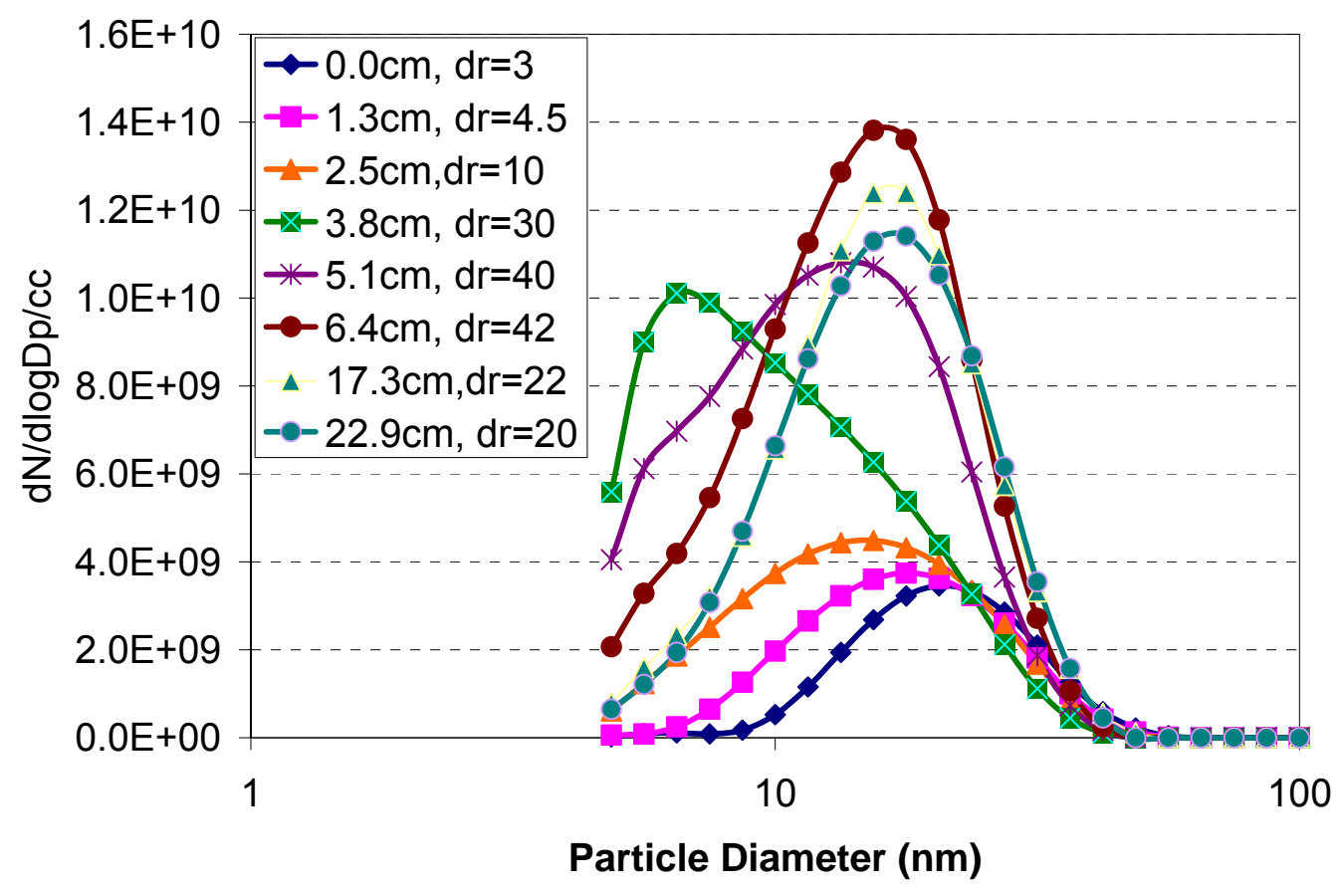

Figure 67 Particle size distributions of the dilution flow at different sampling distance from tunnel cross section center at P1 (primary flow rate $0.378 \mathrm{~m}^{3} / \mathrm{s}, 122$ Nm@1800 rpm)

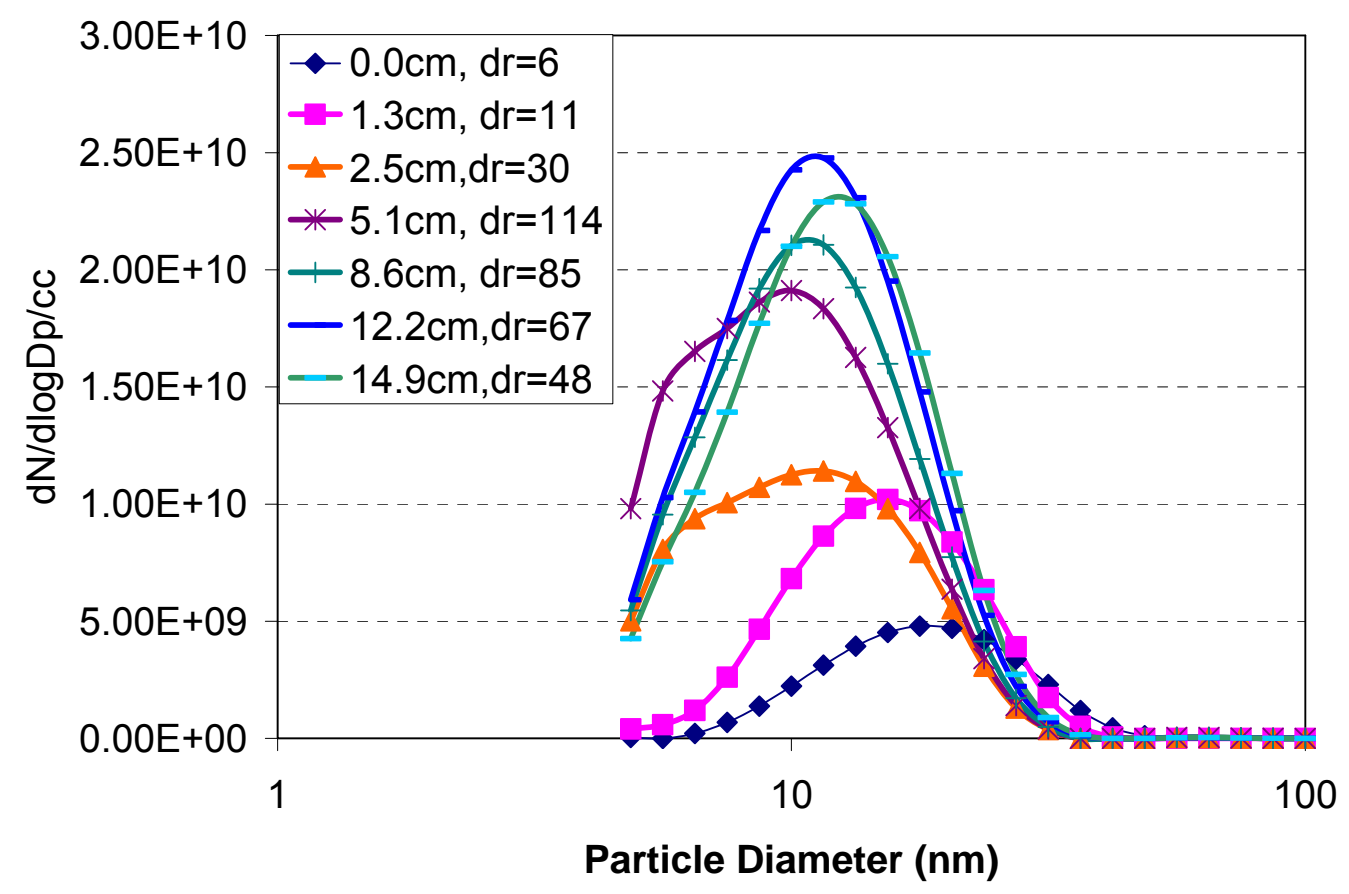

Figure 68 Particle size distributions of the dilution flow at different sampling distance from tunnel cross section center at P1 (primary flow rate $0.944 \mathrm{~m}^{3} / \mathrm{s}, 122$ Nm@1800 rpm) 
Figure 69 and Figure 70 depict particle size distributions of the dilution flow as a function of different sampling distances from the tunnel centerline at P2, for the same tunnel and engine operating conditions as the data collected at P1. Similar bi-modal distribution shifting to single mode patterns was observed at P2. The particle nucleation was also reinforced with higher dilution ratios to generate increased nanoparticle count. Since P2 was further downstream than $\mathrm{P} 1$, and the dilution ratios at the $\mathrm{P} 2$ section center had reached the range in favor of smaller $(<10 \mathrm{~nm})$ particle nucleation, the size distributions of the dilution flow at the P2 section center started with a bi-modal shape, instead of single peak as at P1, and this development continued at off-center locations.

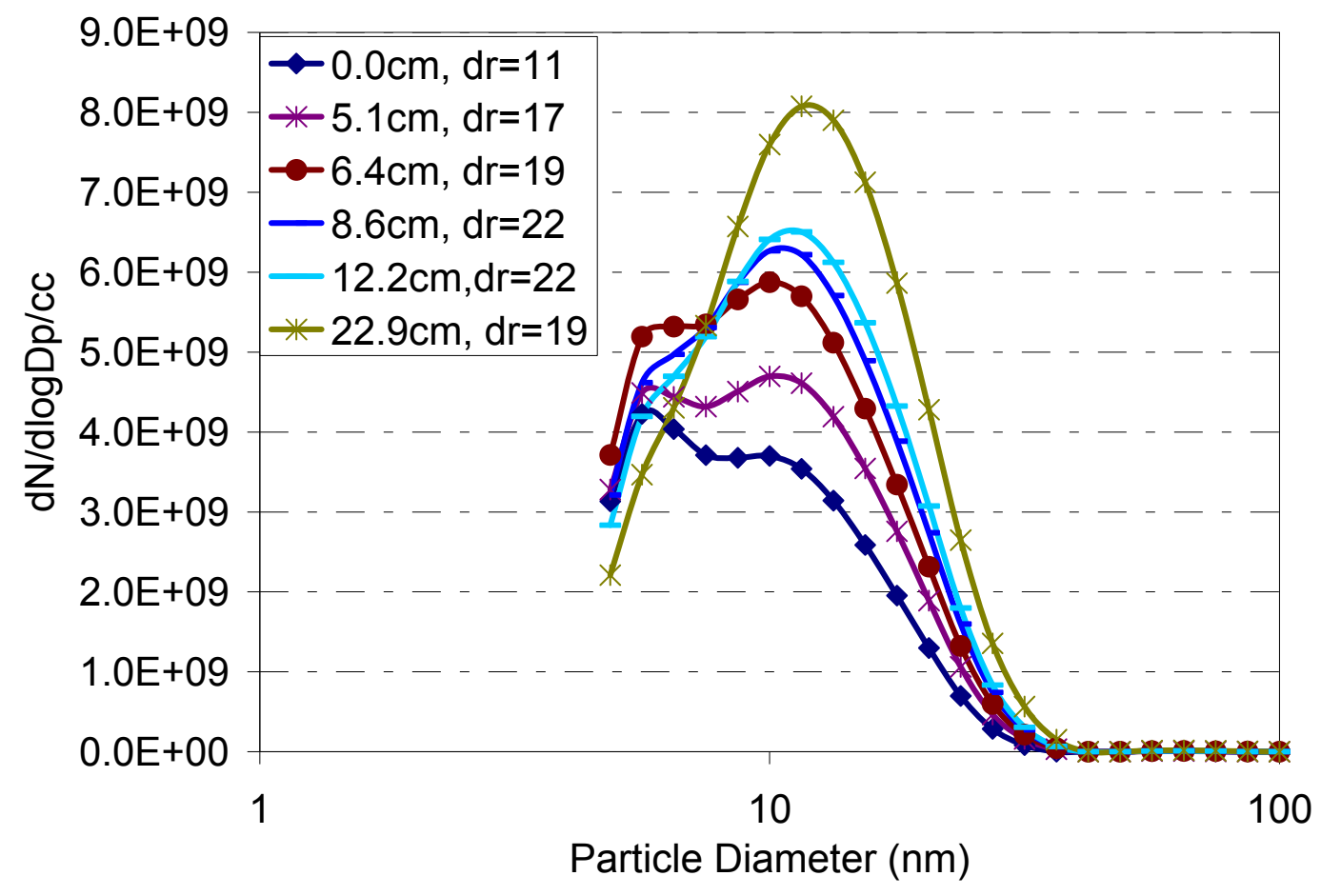

Figure 69 Particle size distributions of the dilution flow at different sampling distance from tunnel cross section center at $P 2$ (primary flow rate $0.378 \mathrm{~m}^{3} / \mathrm{s}, 102$ Nm@1800 rpm) 


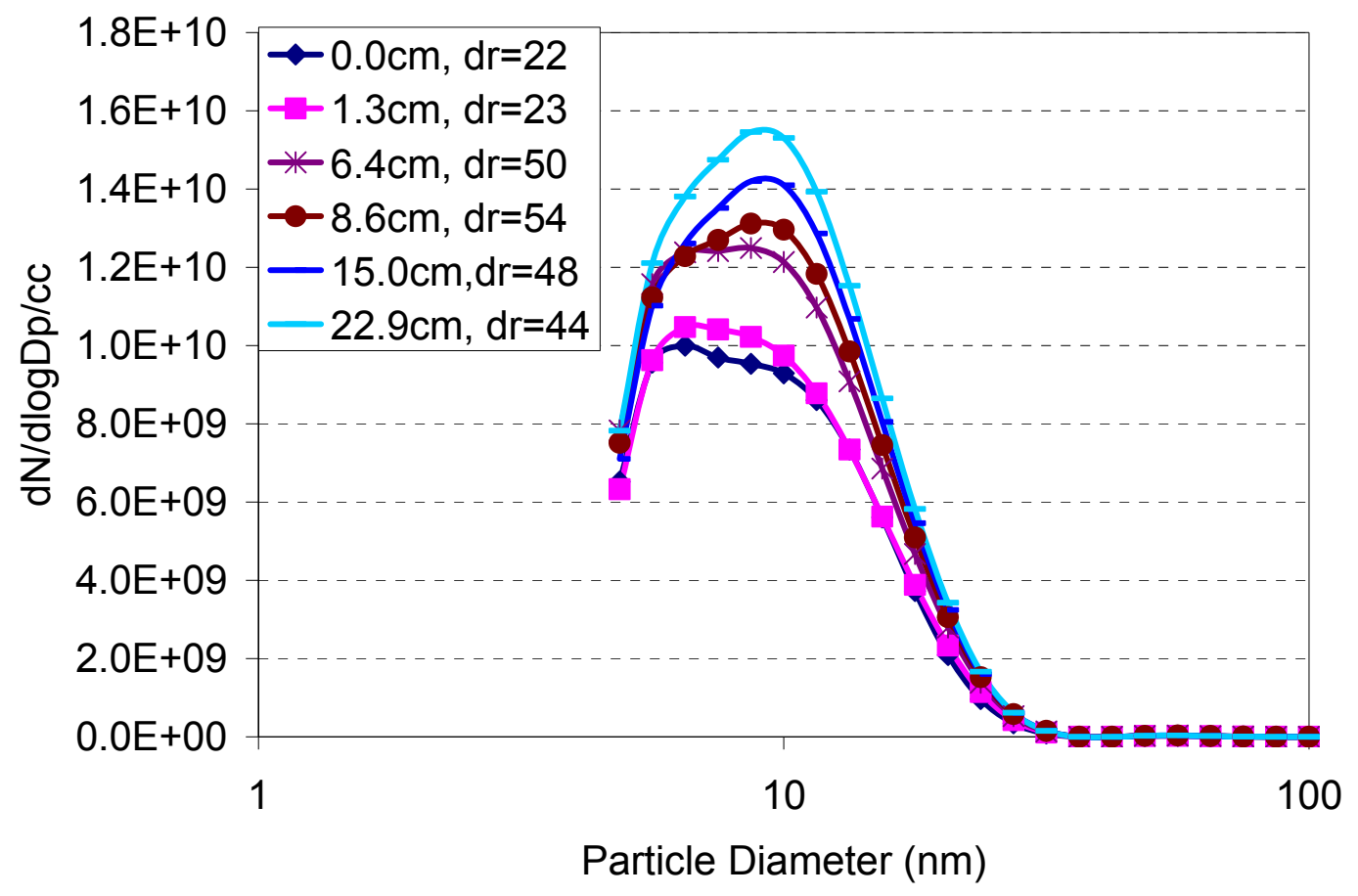

Figure 70 Particle size distributions of the dilution flow at different sampling distance from tunnel cross section center at P2 (primary flow rate $0.944 \mathrm{~m}^{3} / \mathrm{s}, 102$ Nm@ 1800 rpm)

Hydrocarbon species in the gas phase may transit to the particle phase, through processes of adsorption and nucleation, and be characterized as part of the SOF of the DPM [8, 48, and 175]. Adsorption occurs when the SOF accumulates onto existing carbon particles, increasing the size and mass of those particles. Nucleation is responsible for the formation of nano-particles through localized thermodynamic phase change, and increases particle count. Nucleation of SOF may happen in two ways: homogeneous nucleation and heterogeneous nucleation [48]. Homogeneous nucleation occurs when SOF condenses to form new particles. Heterogeneous nucleation occurs when the SOF condenses onto surface of available nuclei. Compared to homogeneous nucleation, which requires high super-saturation values of SOF vapor to trigger the process, heterogeneous nucleation can occur at much lower saturation values. It is important to note that the dilution process is not uniform in the tunnel, and that the degree of mixing will vary in space and in time. The history of each "packet" of exhaust gas, as it mixes with the dilution air, will influence the degree of phase change which occurs, as well as the size distribution arising from the phase change. Absorption and nucleation are considered to 
be two competing, processes [48]. When more SOF is absorbed onto the existing carbon particles, less SOF is available for promotion of nucleation. Conversely, when the exhaust flow is filtered by a DPF to remove most carbonaceous soot, the nucleation mode becomes the dominant process for DPM formation, due to absence of carbonaceous surfaces on which SOF may adsorb. Other factors may also affect measured DPM size distributions, such as particle diffusion, particle impactions onto the orifice plate or tunnel walls, particle coagulation, SOF evaporation and sample probe intrusion.

Nucleation processes in diluted exhaust flow are driven by SOF concentration and temperature, and their respective local gradients. Since the research reported herein was conducted using an engine operating at steady-state conditions, dilution ratios and their rates of change acted as direct indicators of the average history of SOF concentration and temperature, and their associated gradients. Dilution ratios played an important role in triggering lower-end peaks of particle size distributions, and the rate of dilution was also important for smaller particle nucleation. Measured dilution ratios, as a function of distance from centerline, are illustrated in Figure 18 and Figure 64. These data suggest that changes in dilution ratios, depicted as profile curves at $\mathrm{P} 1$ and $\mathrm{P} 2$, are greater nearer the tunnel centerline, whereas dilution ratio was more constant closer to the tunnel wall. In other words, dilution processes occurred, on average, at a faster rate near the tunnel centerline, although local dilution processes are acknowledged to be stochastic.

In addition to the dilution ratio and rate of dilution (i.e. dilution history), the characteristics of engine emissions also had an effect on the particle size distribution shapes. The bi-modal distribution observed in Figure 69 and Figure 70, at P2, disappeared when the engine was running at a higher load (122 Nm). Figure 71 and Figure 72 shows the particle size distributions at P2 when the engine was running 122 $\mathrm{Nm}$ with $0.378 \mathrm{~m}^{3} / \mathrm{s}$ and $0.944 \mathrm{~m}^{3} / \mathrm{s}$ primary flows. The particle size distributions exhibited only one peak, and no obvious peak shifting was observed across the section. However, the presence of higher dilution ratios at various locations was still assumed to generate more nano-particles when the exhaust flow was fast diluted. 


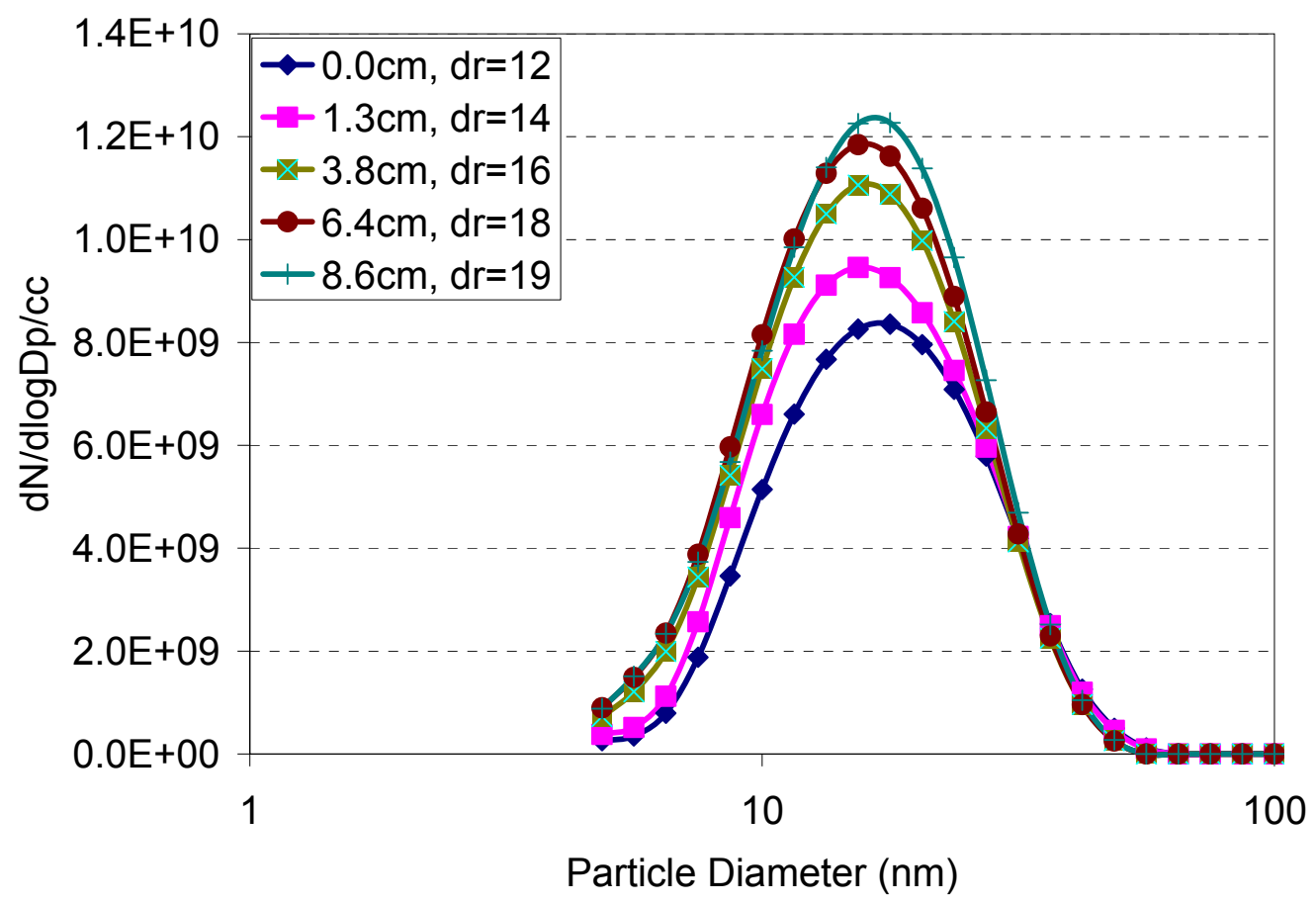

Figure 71 Particle size distributions of the dilution flow at different sampling distance from tunnel cross section center at P2 (primary flow rate $0.378 \mathrm{~m}^{3} / \mathrm{s}, 122$ Nm@ @ 1800 rpm)

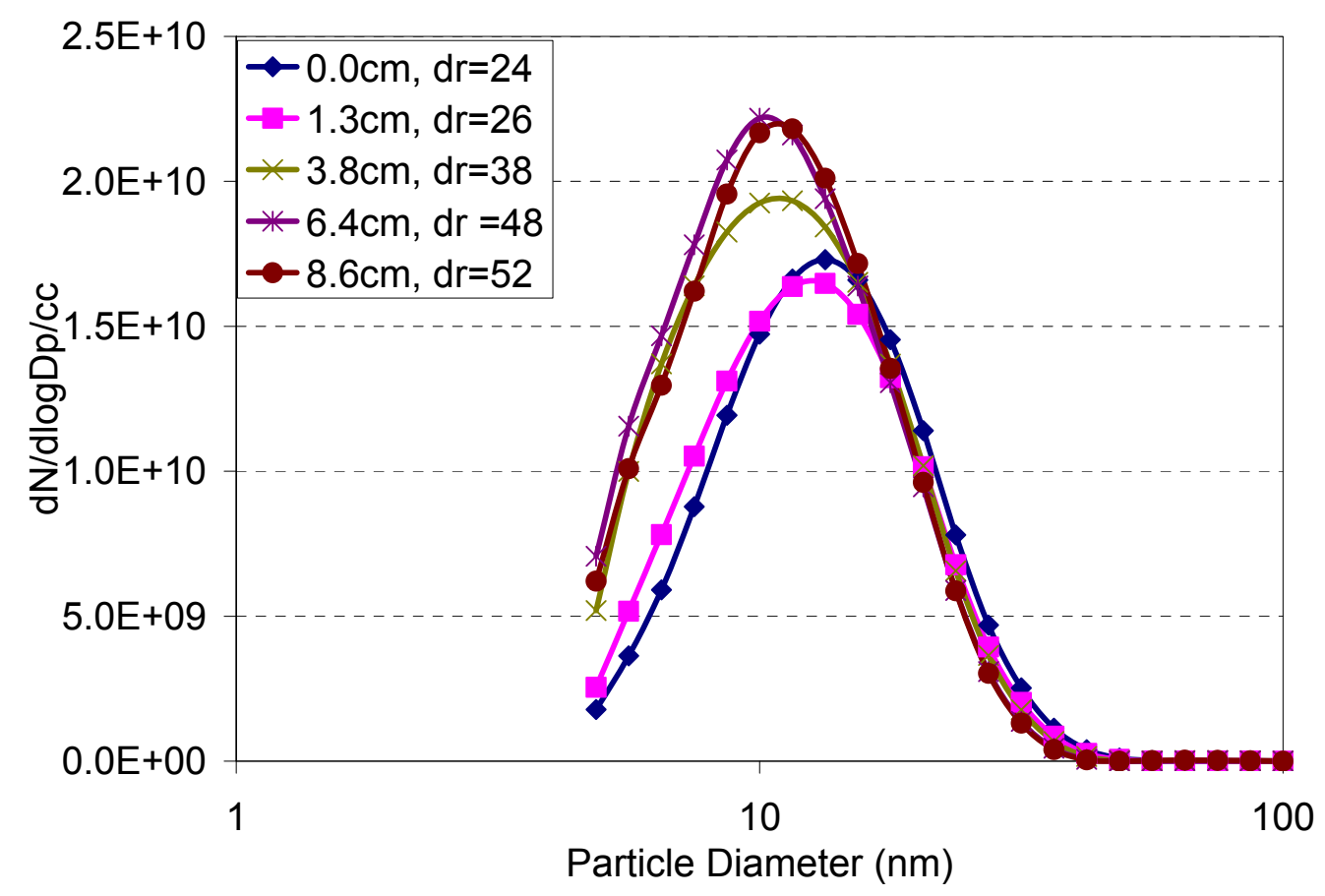

Figure 72 Particle size distributions of the dilution flow at different sampling distance from tunnel cross section center at P2 (primary flow rate $0.944 \mathrm{~m}^{3} / \mathrm{s}, 122$ Nm@1800 rpm) 
Residence time had a significant influence on the Diesel particle characteristics. Since mixing occurs in a turbulent fashion, the residence time is truly characterized by a distribution, along with a distribution of concentration and thermal histories, but average values are still valuable to consider. Once the flow was fully mixed, the measured dilution ratios were constant, and particle size distributions did not change appreciably across the tunnel cross-sections. Measured particle size distributions at P3 and P4, with different primary flow rates, provide necessary information regarding the influence of residence time and primary dilution ratios on Diesel particle size. Measurements of particle size distributions across $\mathrm{P} 3$ and $\mathrm{P} 4$ sections were collected while the engine was maintained at $102 \mathrm{Nm}$ at $1800 \mathrm{rpm}$ with primary flow rates of $0.378,0.566,0.755,0.944$, and $1.180 \mathrm{~m}^{3} / \mathrm{s}$. Figure 73 shows the results of particle size distribution measurements with 3 different primary flow rate settings of $0.378,0.755$, and $1.180 \mathrm{~m}^{3} / \mathrm{s}$. The corresponding average residence time from $\mathrm{P} 3$ to $\mathrm{P} 4$ varied from $1.3 \mathrm{~s}$ to $0.4 \mathrm{~s}$, for those three primary flow rates. It can be seen that the total particle count decreased between locations P3 and P4, for all primary flow settings. Meanwhile, the peaks of particle distribution curves shifted 2-3 nm toward larger equivalent diameters between locations P3 and P4. The dilution ratios for the primary flow rate setting $0.378,0.755$, and 1.180 $\mathrm{m}^{3} / \mathrm{s}$ are respectively $12.5,25.0$, and 39.1 , and there was no radial variation of dilution ratio observed across $\mathrm{P} 3$ and $\mathrm{P} 4$ sections. The dilution ratios corresponding to higher primary flow rates displayed higher total particle count. This demonstrated that, within the range of dilution ratios corresponding to the varied primary flow rates, increased particle nucleation was more likely to occur at higher dilution ratios. The shift of particle size distribution peaks from smaller to larger particle mean diameters, along with the reduction of total particle count, indicated that suppressed particle nucleation between location P3 and P4. At the same time, large diameter particles at P4 were measured, likely due to agglomeration. Along size distribution shifts, total particle count decreased by approximately $37 \%, 23 \%$ and $14 \%$ with primary flow rate of $0.378,0.755$, and 1.180 $\mathrm{m}^{3} / \mathrm{s}$, respectively. This tends to suggest that shorter residence times corresponded to less pronounced changes in particle size distributions, since there was less time for the particle transformations to occur. Results obtained at 0.566 and $0.944 \mathrm{~m}^{3} / \mathrm{s}$ flow rates indicated similar patterns, and were omitted in Figure 11 for the sake of brevity. 


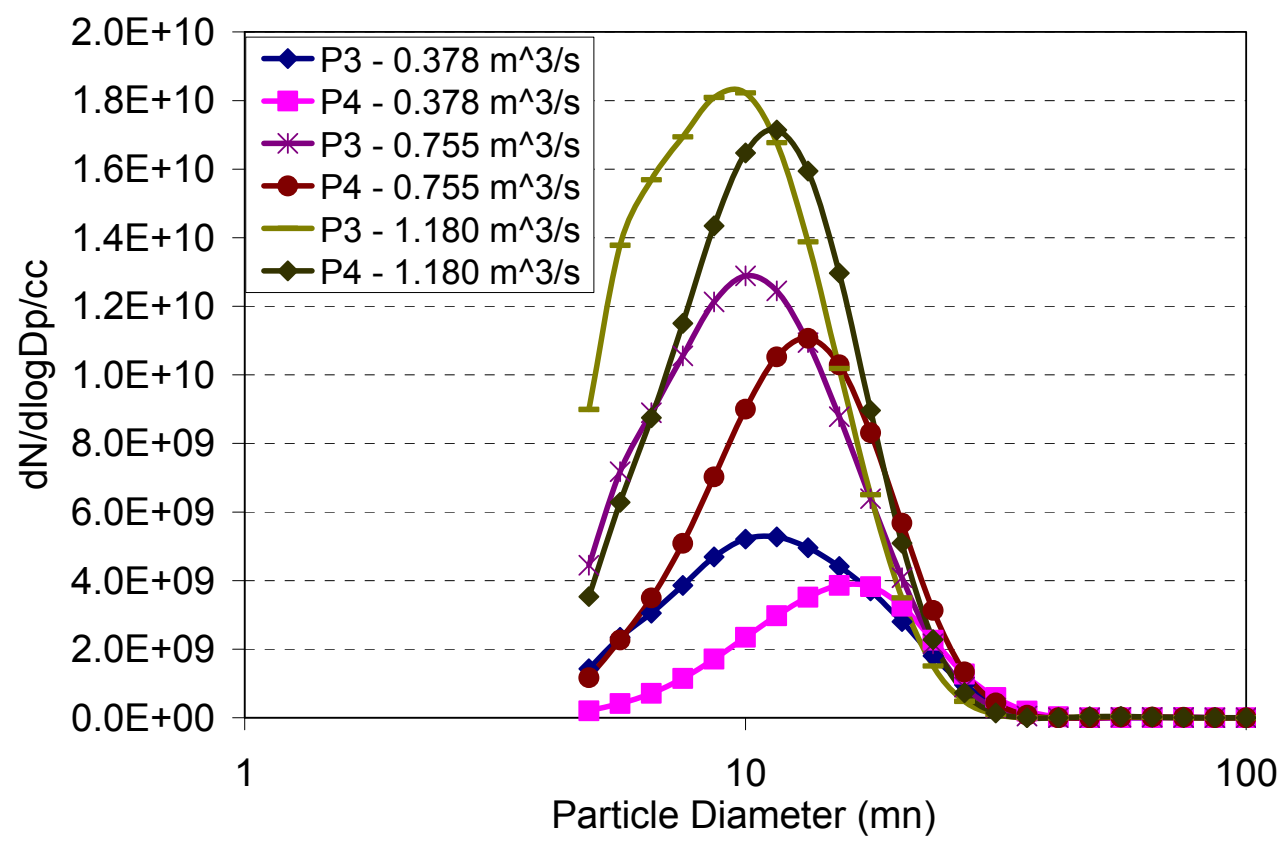

Figure 73 Particle size distributions of the dilution flow at P3 and P4 (primary flow rate $\left.0.378 \mathrm{~m}^{3} / \mathrm{s}, 102 \mathrm{Nm} @ 1800 \mathrm{rpm}\right)$

Exhaust stack equivalent DPM mass concentration levels were also analyzed to investigate the effect that Diesel particle transformations from P3 to P4 would have on total PM mass. Particle mass estimations, from a previous study [176], were obtained as a function of a particle's diameter, and given by:

$$
\operatorname{Mass}(\mu g)=1.54 \times 10^{-16} \times D^{3.19}
$$

Equation 5. 18

where $D$ is the particle diameter in nanometers. PM mass concentrations increased from P3 to P4 for all primary flow rates.

Figure 74 and Figure 75 shows the measured total particle number and mass concentrations versus residence time (uncertainty analysis described in Appendix A.1 was used to calculate the error bars in particle number and mass concentration plots in this dissertation). Values corresponding to residence times $0.4 \mathrm{~s}$ to $1.3 \mathrm{~s}$ were data measured at P3 and P4 inside the primary tunnel while the primary flow rates were set to 0.378 to $1.180 \mathrm{~m}^{3} / \mathrm{s}$. The particle concentration values for residence time $4.5 \mathrm{~s}$ were measured at inlet and out of the secondary tunnel while the primary tunnel flow rate was 
set to $0.378 \mathrm{~m}^{3} / \mathrm{s}$. The result for the secondary tunnel was put here for better interpretation of residence time effect.

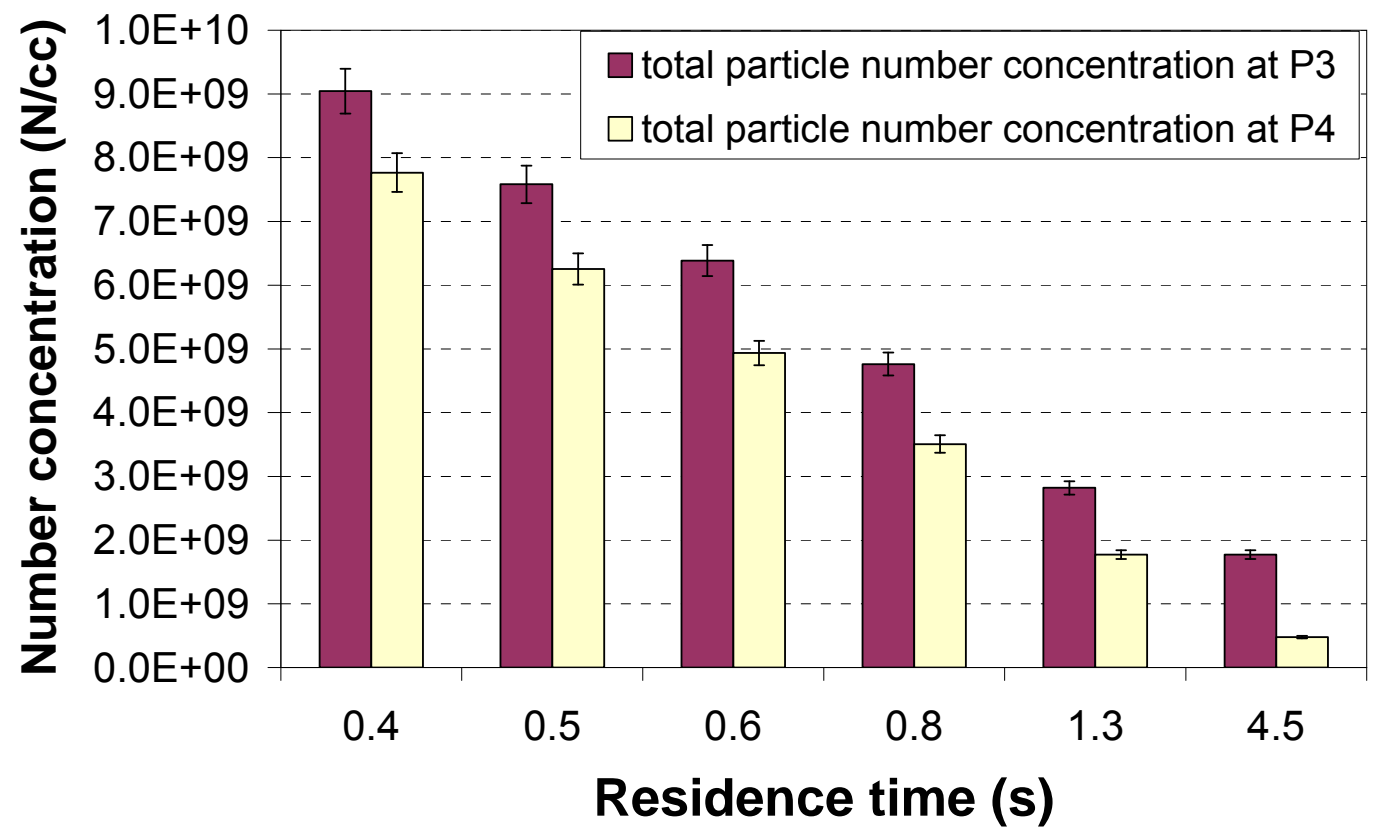

Figure 74 Residence effects on total particle number concentration (102 Nm @ 1800 rpm)

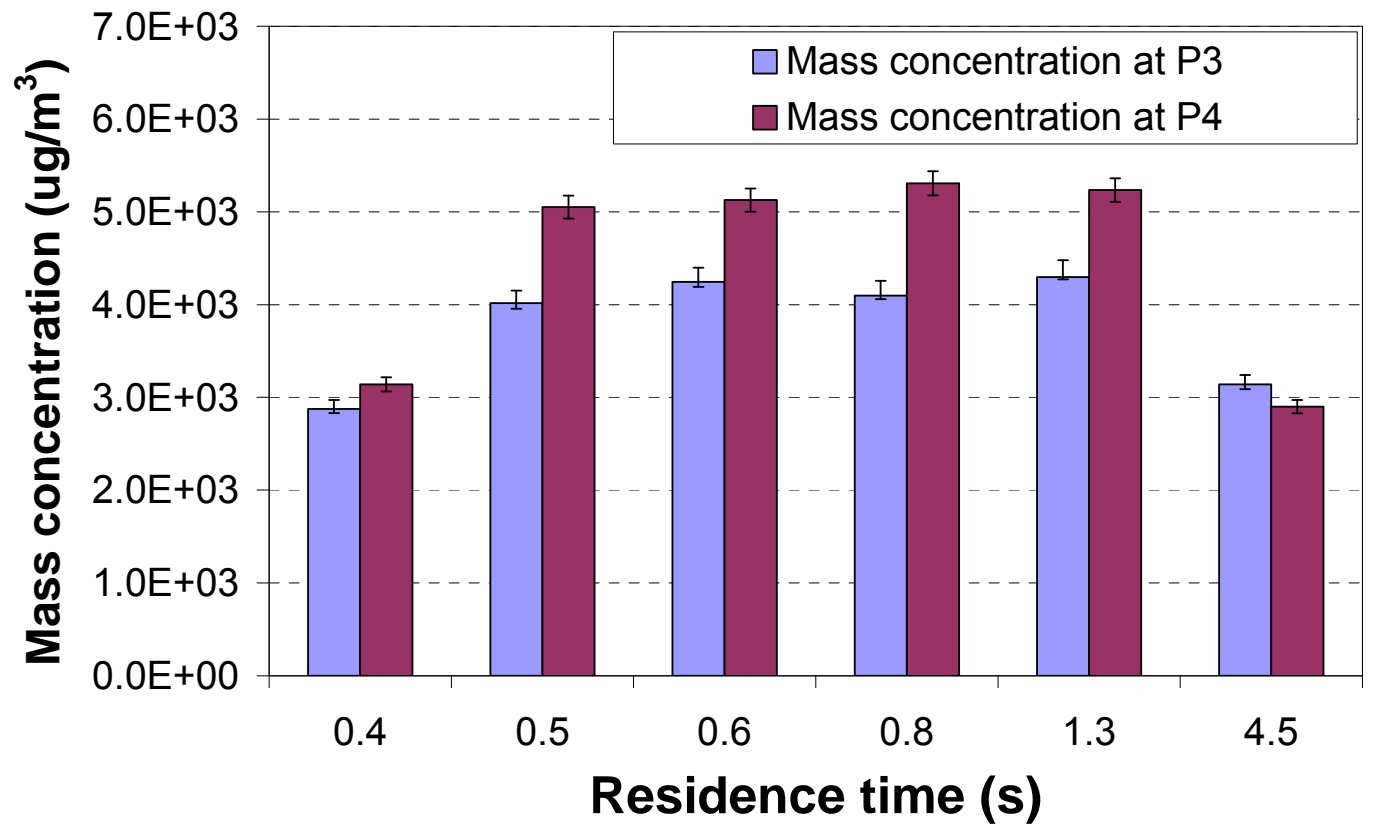

Figure 75 Residence effects on total particle mass concentration (102 Nm @ 1800 rpm) 
In Figure 74 , total particle count reductions of $14 \%, 18 \%, 23 \%, 26 \%$ and $37 \%$ for residence times of $0.4 \mathrm{~s}, 0.5 \mathrm{~s}, 0.6 \mathrm{~s}, 0.8 \mathrm{~s}$ and $1.3 \mathrm{~s}$ were measured in the primary tunnel between locations P3 and P4. Investigation of residence time effects of the secondary tunnel indicated a $73 \%$ reduction in number count associated with a residence time of 4.5s (total secondary flow $56.6 \mathrm{lpm}$ ), with dilution ratio of 1 . However, further increased residence time, obtained by decreasing total secondary flow rates, exhibited no measurable effects on particle size distributions. Particle size distributions with residence times between $12 \mathrm{~s}(21.2 \mathrm{lpm}$ total flow rate) and $36 \mathrm{~s}(7.1 \mathrm{lpm}$ total flow rate) were practically identical.

Figure 75 revealed that mass concentration levels at P3 or P4 changed less from the primary flow rate of $0.566 \mathrm{~m}^{3} / \mathrm{s}$ to $1.180 \mathrm{~m}^{3} / \mathrm{s}$ than those from $0.378 \mathrm{~m}^{3} / \mathrm{s}$ to 0.566 $\mathrm{m}^{3} / \mathrm{s}$. This indicated that dilution ratio had less effect on PM mass concentration levels after a threshold of primary dilution ratio was reached (here with the primary flow rate between $0.378 \mathrm{~m}^{3} / \mathrm{s}$ and $0.566 \mathrm{~m}^{3} / \mathrm{s}$ ). When the effects of the residence time were examined, the percentage of increased mass concentration levels between P3 and P4 was smallest $(10 \%)$ for $0.378 \mathrm{~m}^{3} / \mathrm{s}$ among all primary flow rates. Mass percentage increases were between $20 \%$ and $30 \%$ for the primary flow rate settings from $0.566 \mathrm{~m}^{3} / \mathrm{s}$ to 1.180 $\mathrm{m}^{3} / \mathrm{s}$, with the maximum mass concentration level (occurring at P4@1.180 m³ $/ \mathrm{s}$ ) being about 2 times that of the minimum mass concentration level observed (occurred at P4@0.944 m³ 3 ).

The total particle mass concentration increased for residence times from $0.4 \mathrm{~s}$ to $1.3 \mathrm{~s}$, as shown in Figure 75. When such increases in particle mass occur in sympathy with particle size distribution shifts, one must consider that particle coagulation and condensation could have acted as the dominant particle dynamic process. Particle coagulation is the growth of particles through collisions with other particles, wherein total particle mass is conserved. Coagulation will, however, reduce total particle number and shift the particle size distribution. Condensation is the process of SOF changes from gas to solid phase by nucleation or absorption, and increases total particle mass. It can be seen from Figure 74 and Figure 75 that for residence times from $0.4 \mathrm{~s}$ to $1.3 \mathrm{~s}$, a marked 
reduction in total particle number concentration was realized, indicating that the most dominant particle dynamic was, likely, coagulation. The second-most important process would be SOF condensation, which increased total particle mass concentration values. Figure 74 also indicated that as residence time increased, coagulation processes exhibited stronger influence in particle number concentration. Conversely, condensation processes were more stable than coagulation processes for residence times from $0.5 \mathrm{~s}$ to $1.3 \mathrm{~s}$, as particle mass values increased slightly. For particle size distributions measured for a $4.5 \mathrm{~s}$ residence time in the heated secondary tunnel, particle coagulation is still considered the dominant process, as largely reduced number concentrations and slightly reduced mass concentrations are indicated (Figure 74 and Figure 75). However, the loss of total particle mass for a residence time of $4.5 \mathrm{~s}$ illustrated that the processes of particle diffusion and SOF evaporation might have been stronger than SOF condensation, since reductions of both particle number and mass concentration were reported.

\subsubsection{Secondary Tunnel}

Results for engine loads of $102 \mathrm{Nm}$ are shown in Figure 76, and, for brevity, the nearly identical data obtained from the tests at $122 \mathrm{Nm}$ were not included. Since sampling port P4 was located within $12.3 \mathrm{~cm}$ upstream of the sampling probe of the secondary tunnel, the particle size distribution measured at P4 section can be regarded as being representative of those at the inlet of the probe for the secondary dilution tunnel. Results indicated that the mean particle diameter increased approximately $5 \mathrm{~nm}$ as a result of even a small amount of injected dilution air. When dilution ratios were controlled to less than 3, particle size distributions measured at the sampling position, exhibited higher, narrower, and smaller diameter peaks, compared to those obtained at the secondary tunnel inlet. However, no significant changes $(>10 \%)$ of the total particle count were observed at the secondary outlet with dilution ratios less than 3 . When secondary dilution ratios were controlled between 3 and 9, mean particle equivalent diameters decreased, and the total particle count decreased consistently. At a secondary dilution ratio of 9 , particle count had reduced to $40 \%$ of that measured at the secondary tunnel inlet. 


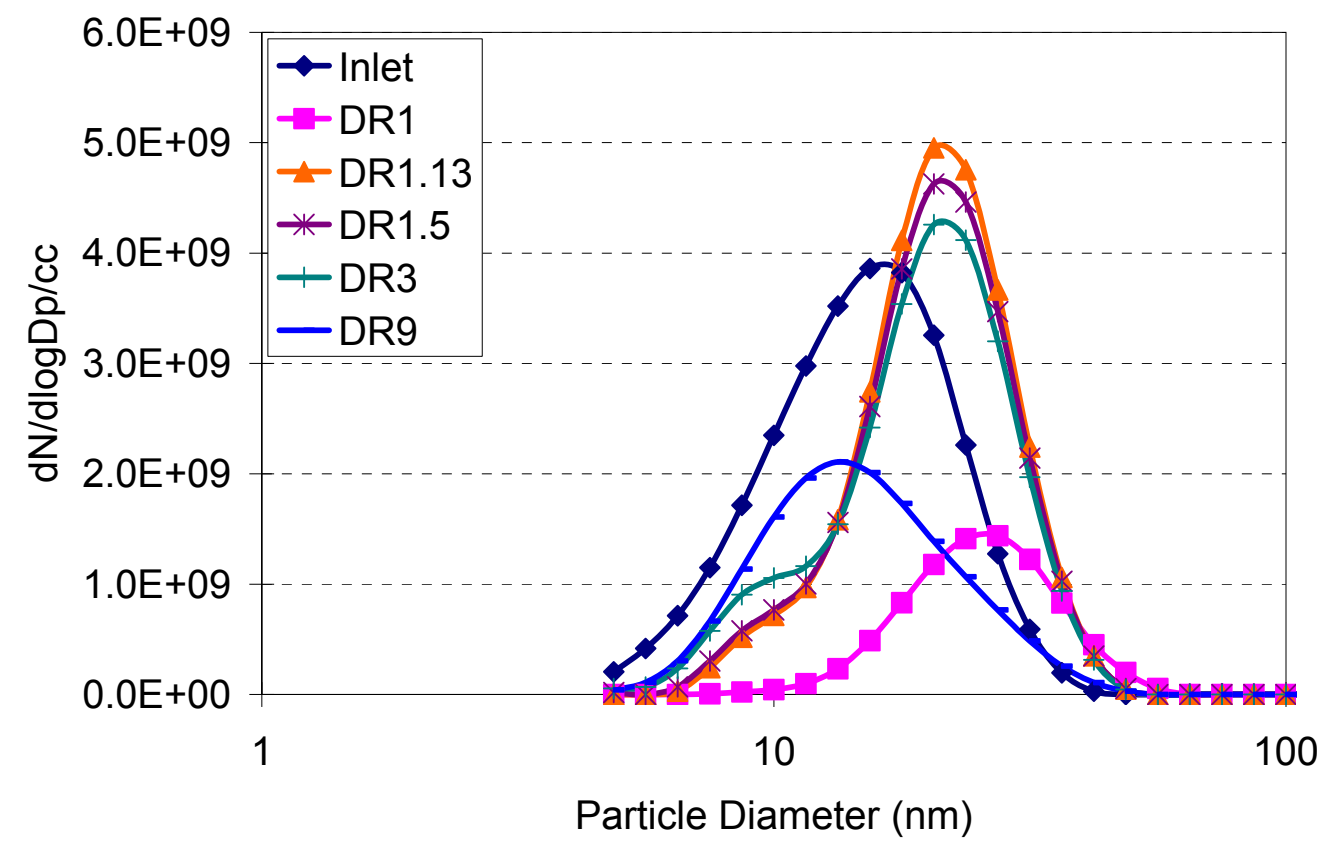

Figure 76 Particle size distributions at the end of the secondary tunnel with different secondary dilution ratio (primary flow rate $0.378 \mathrm{~m}^{3} / \mathrm{s}, 102 \mathrm{Nm} @ 1800$ rpm)

With no secondary dilution (secondary dilution ratio equal to 1), measured particle size distributions showed a significant (73\%) reduction of total particle count, with a mean diameter reduced by $10 \mathrm{~nm}$, compared to the distribution measured at the secondary tunnel inlet. This phenomenon was similar to the particle size distribution behavior identified in the primary dilution tunnel between P3 and P4 (see Figure 73). Since the secondary tunnel flow was heated to $47^{\circ} \mathrm{C}$ at the outlet of the secondary tunnel, the final shape of the particle size distribution at the tunnel outlet was the result of the combined effects of the residence time and the tunnel wall heating. Further analysis indicated that the most influential of these was indeed particle residence time as shown in Figure 74. As an example, for a residence time of approximately $4.0 \mathrm{~s}$ in an undiluted secondary tunnel, a $70 \%$ reduction in total particle count, with a shifted mean equivalent diameter was observed. These results, scaled consistently with those obtained from the residence time study of particles in the primary tunnel.

PM mass concentration levels, for the particle size distributions shown in Figure 77 , were corrected for dilution ratios and used to analyze the effects of varied secondary 
dilution ratios on estimated total PM mass. The same mass estimate equation, used previously for generating Figure 12, was employed, and the results are shown in Figure 14. Mass concentration estimates reported for secondary dilution ratios between 1.13 and 3 had an average value of $6,610 \mu \mathrm{g} / \mathrm{m}^{3}$ with uncertainty level $(+217-130) \mu \mathrm{g} / \mathrm{m}^{3}$. When the secondary flow was diluted, the highest mass concentration level observed at a dilution ratio equal to 1.8 was approximately 4 times those obtained with a dilution ratio of 9. This would equate to a $4 \mathrm{x}$ difference in total PM mass collected, assuming that PM particle capture efficiencies of the collection media were constant for equivalent flow rates at varying dilution ratios. The significant loss of particle mass at dilution ratio 9, compared to masses at lower dilution ratios, might be attributed to excessive SOF evaporation with low vapor pressures and tunnel heating. The fact that this mass difference would not likely be measured is a strong indication of the inaccuracies associated with a particle density estimate. However, particle nucleation, and later loss of volatile components during post test conditioning, would likely be observed, and would ultimately exhibit themselves in mass differences and increased test-to-test variation.

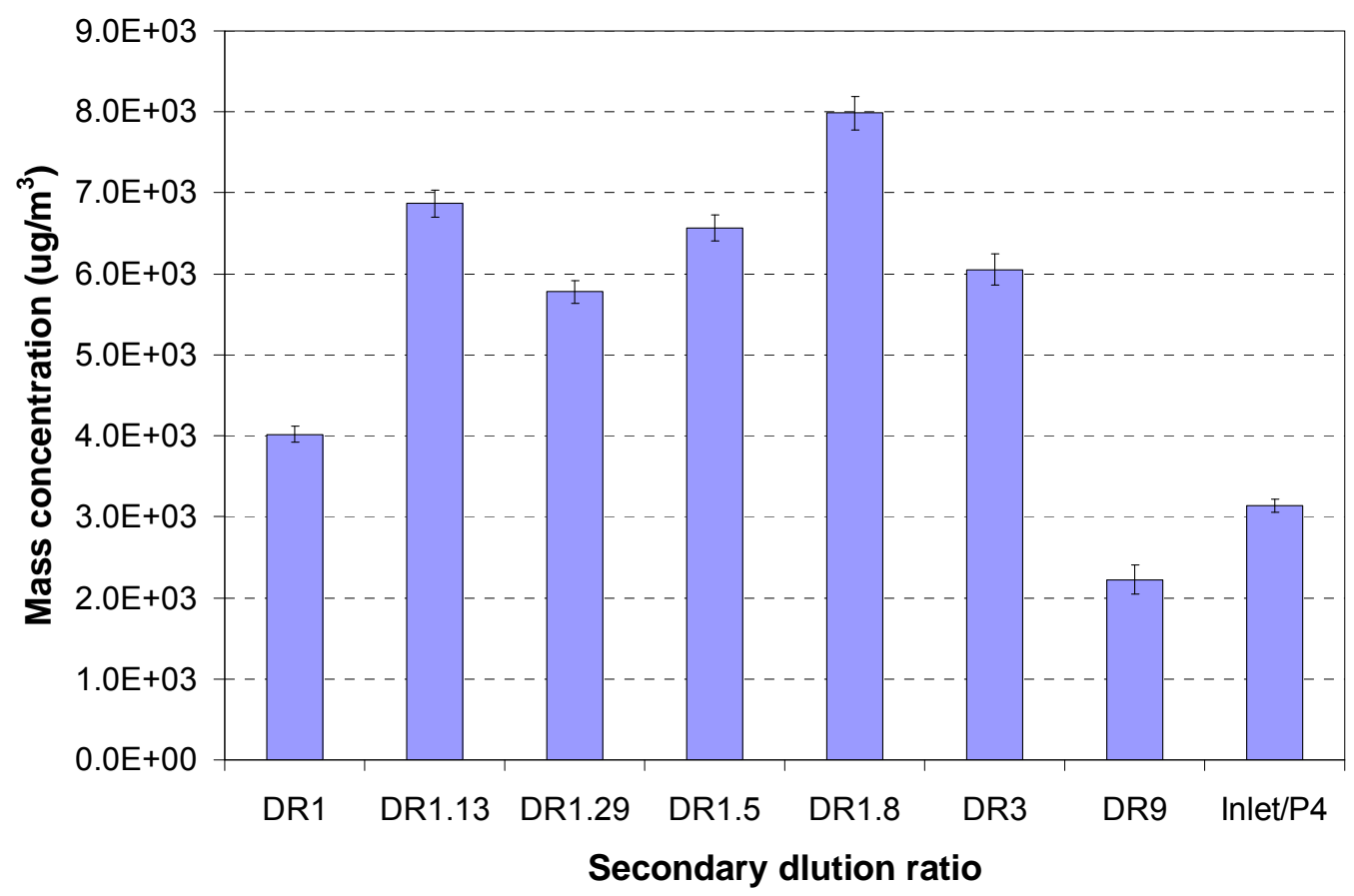

Figure 77 PM mass concentrations with varied secondary dilution ratios (primary flow rate $\left.0.378 \mathrm{~m}^{3} / \mathrm{s}, 102 \mathrm{Nm} @ 1800 \mathrm{rpm}\right)$ 


\subsection{PPMD Test}

The PPMD was configured to operate at constant dilution ratios in single dilution stage mode and double dilution stage mode, with one MPS diluter and two MPS diluters respectively. The particle size distributions measured in single dilution stage mode are shown in Figure 78 and Figure 79. The particle size distributions measured in double dilution stage mode are shown in Figure 80 and Figure 81. These figures show the trend of higher size distribution curve peaks with higher dilution ratios. However, the peak of particle size distribution curve at a dilution ratio of 10 was higher than the peak at dilution ratio of 12 as shown in Figure 78. Figure 79 shows the highest curve peak at dilution ratio equaled to 8 . Figure 80 showed the peaks of particle size distribution curves shifted about $10 \mathrm{~nm}$ to the smaller diameter side compared peaks shown in Figure 78. However, such a shifting was not observed when the engine was operated at $122 \mathrm{Nm}$.

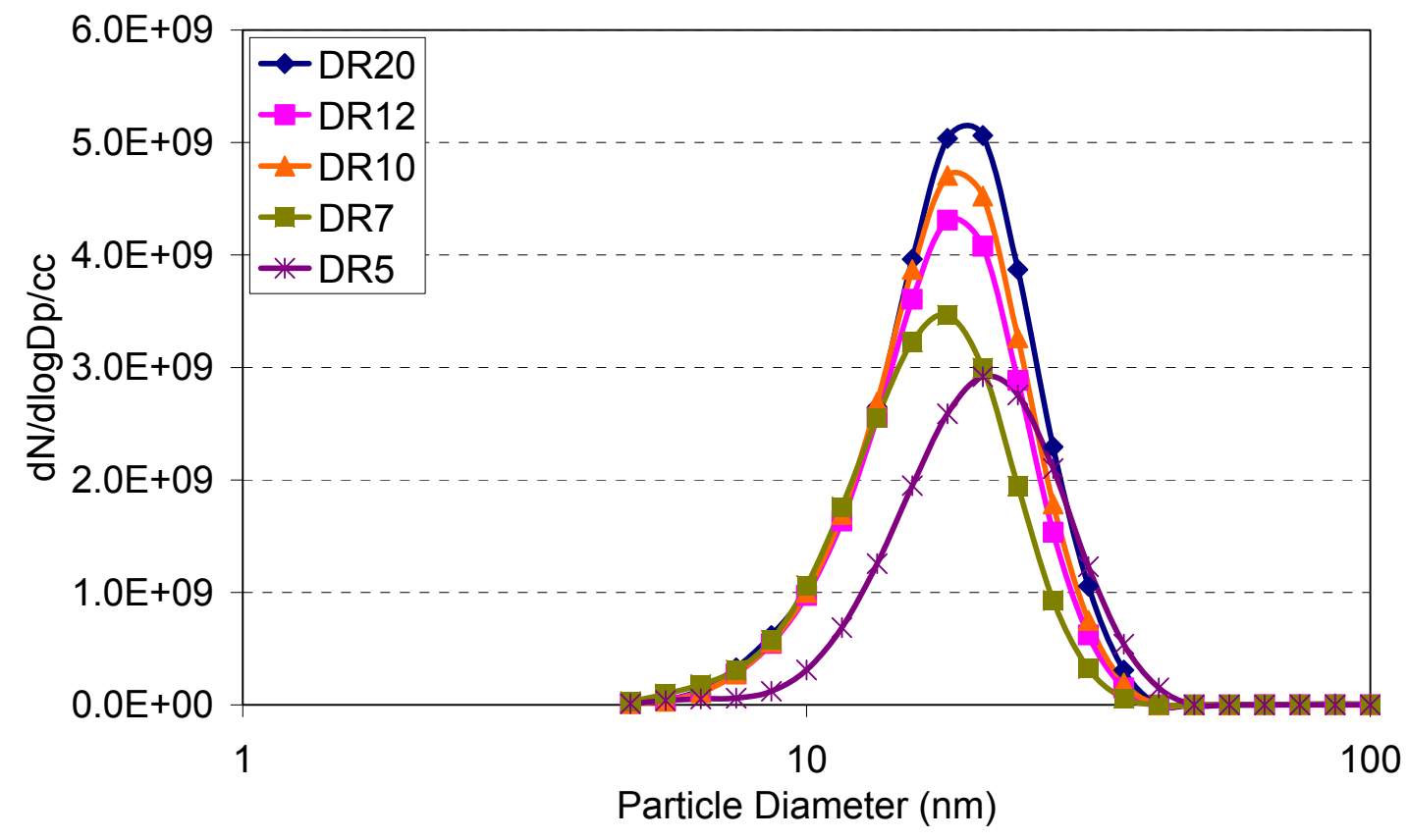

Figure 78 Particle size distributions with single MPS dilution stage (102 Nm @ 1800 rpm) 


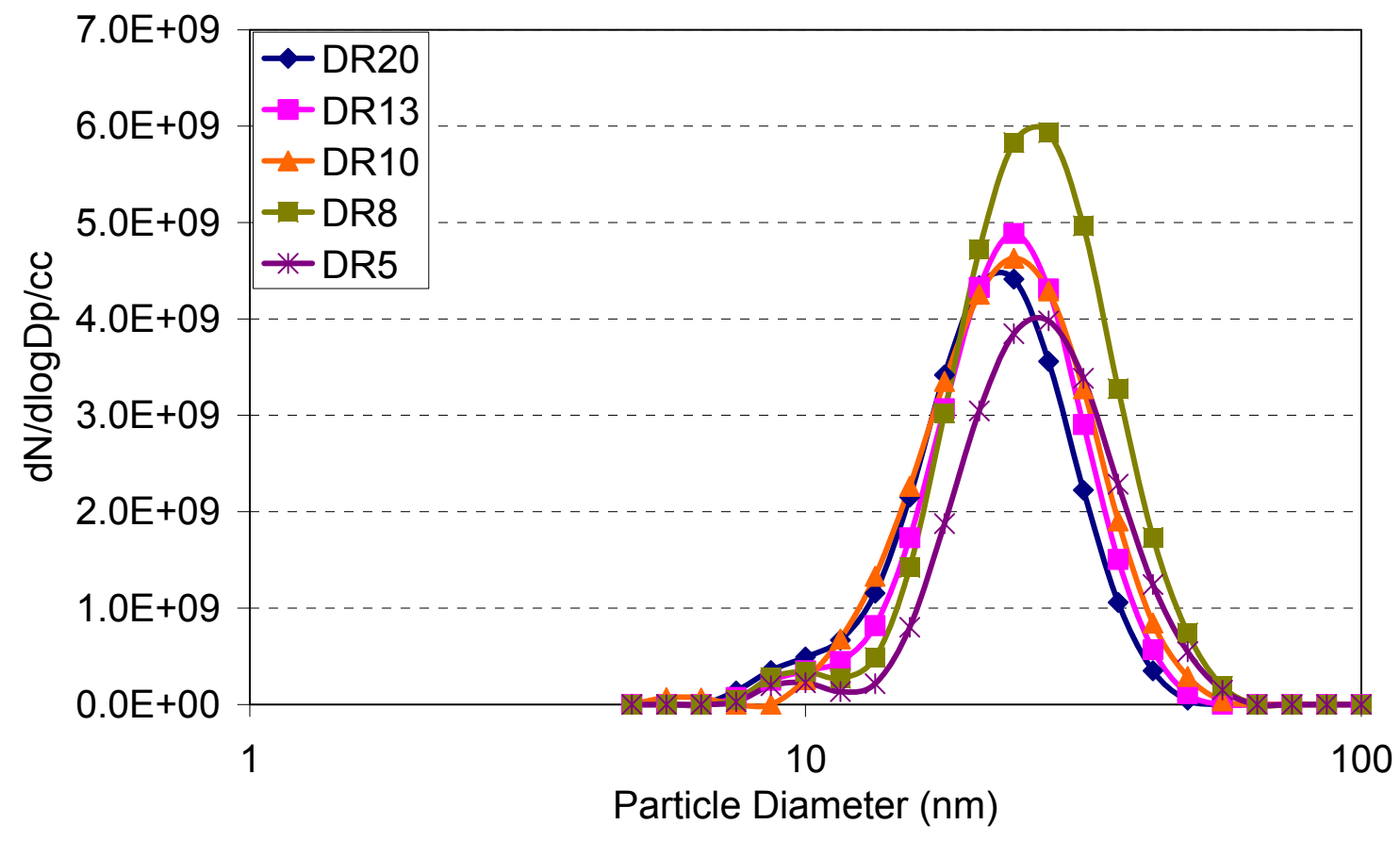

Figure 79 Particle size distributions with single MPS dilution stage (122 Nm @ 1800 rpm)

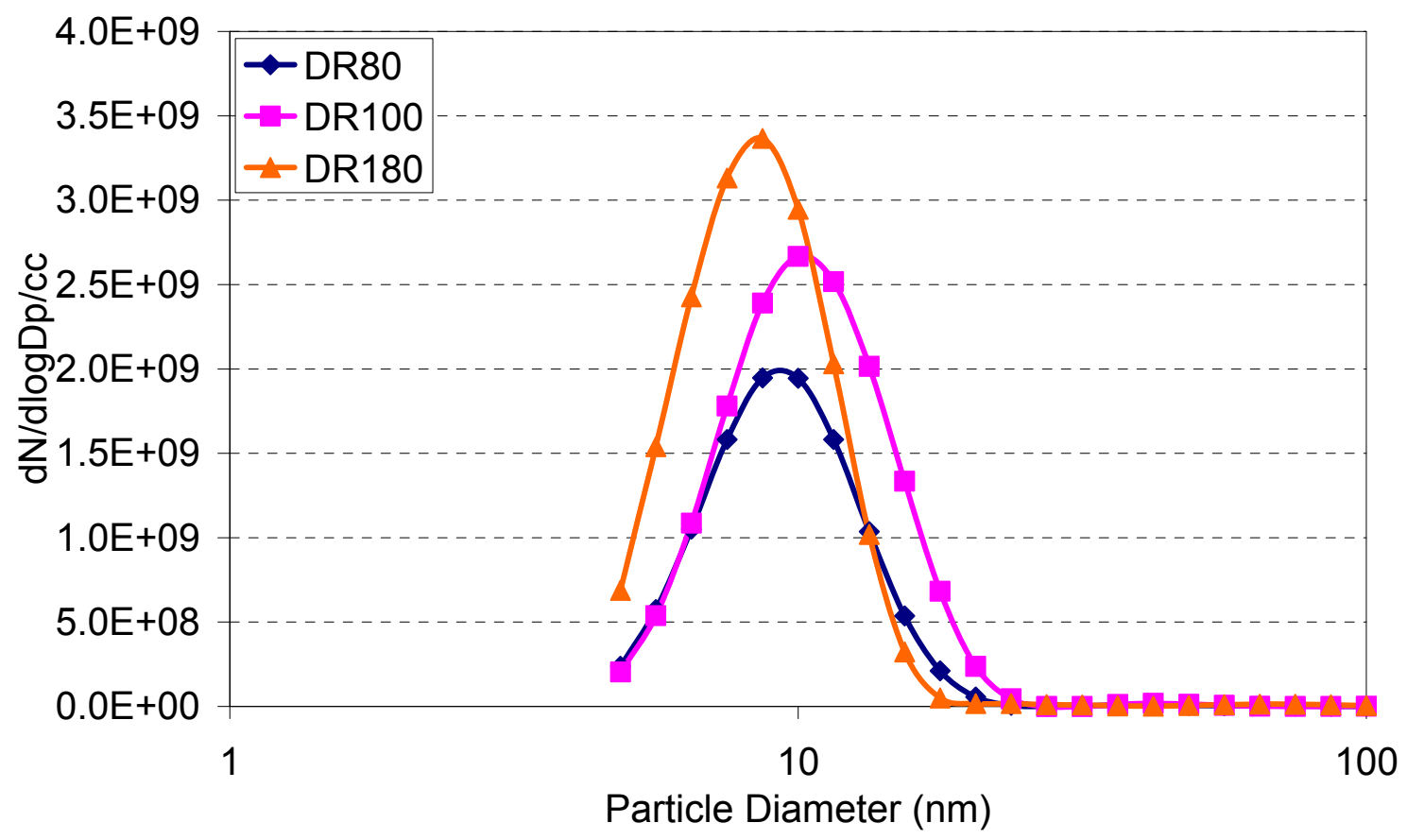

Figure 80 Particle size distributions with double MPS dilution stage (102 Nm @ 1800 rpm) 


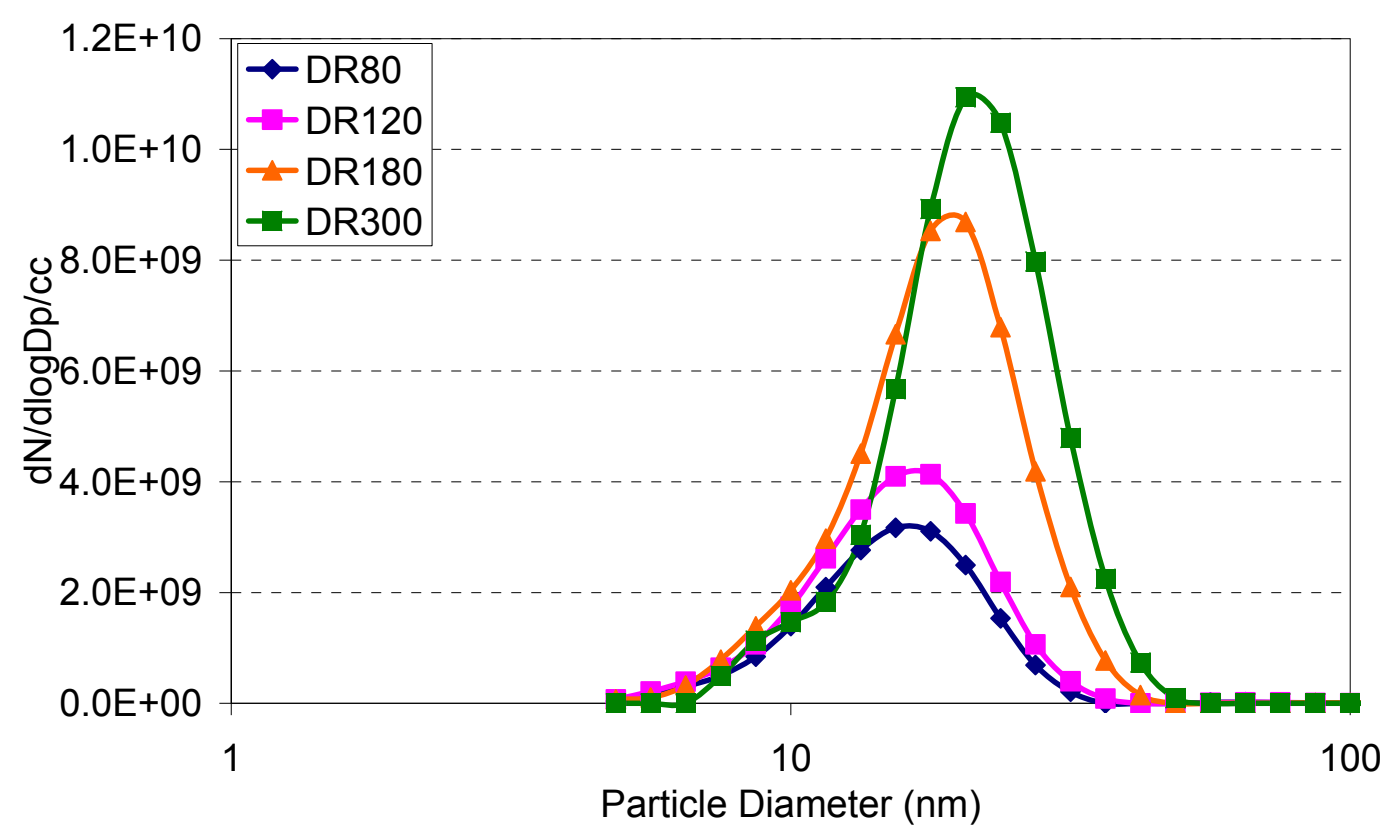

Figure 81 Particle size distributions with double MPS dilution stage (122 Nm @ $1800 \mathrm{rpm})$

The effects of PPMD dilution ratio on the stack equivalent total particle number concentration and particle mass concentration were also examined. The results of analyzed data for two different engine running conditions are shown in Figure 82, Figure 83, Figure 84, and Figure 85.

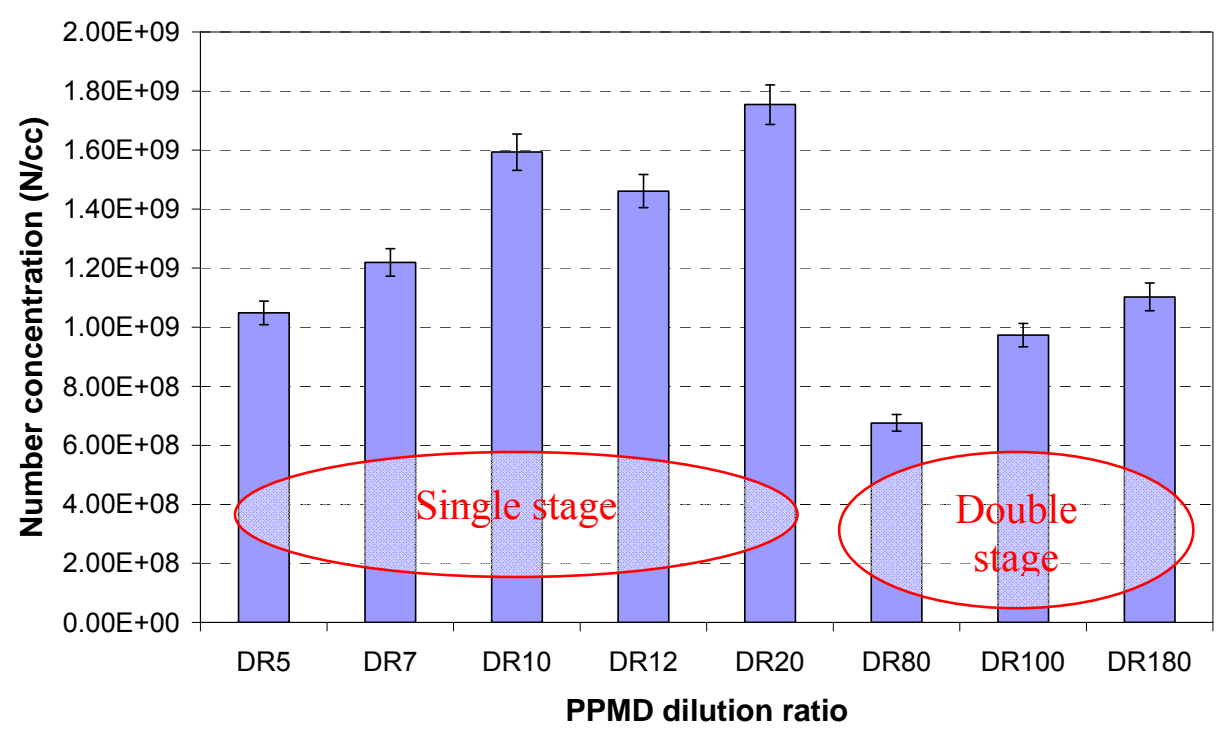

Figure 82 Particle number concentration with PPMD dilution (102 Nm @1800 rpm) 


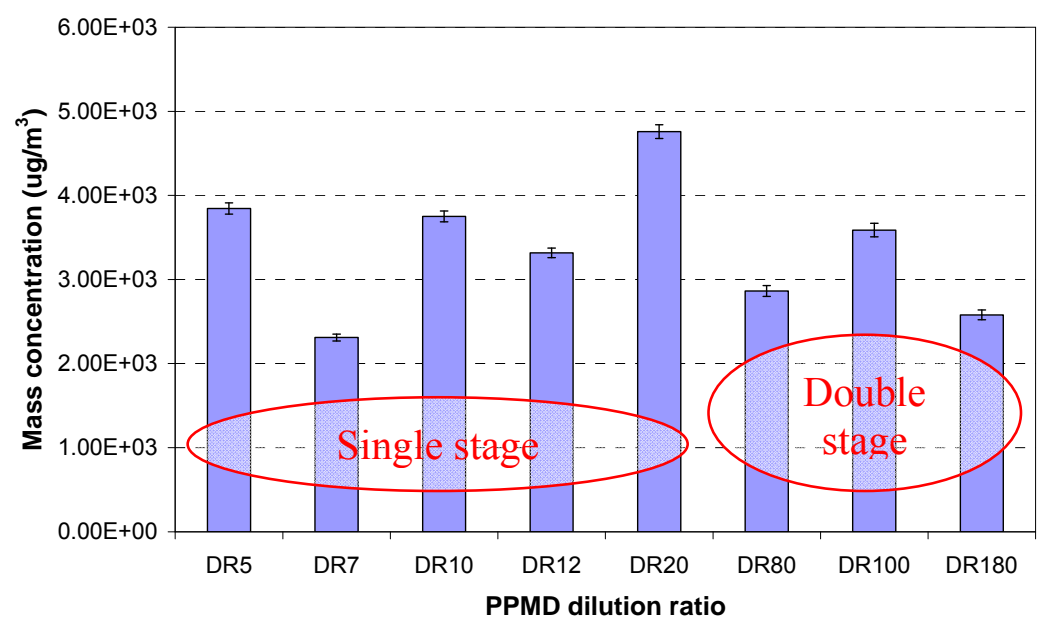

Figure 83 Particle mass concentration with PPMD dilution (102 Nm @1800 rpm)

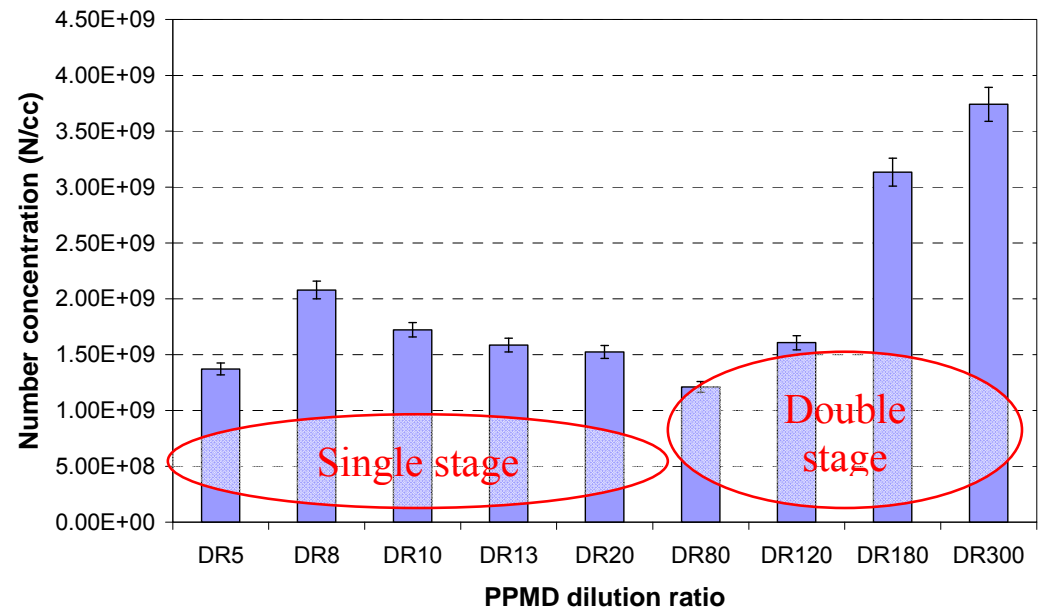

Figure 84 Particle number concentration with PPMD dilution (122 Nm @1800 rpm)

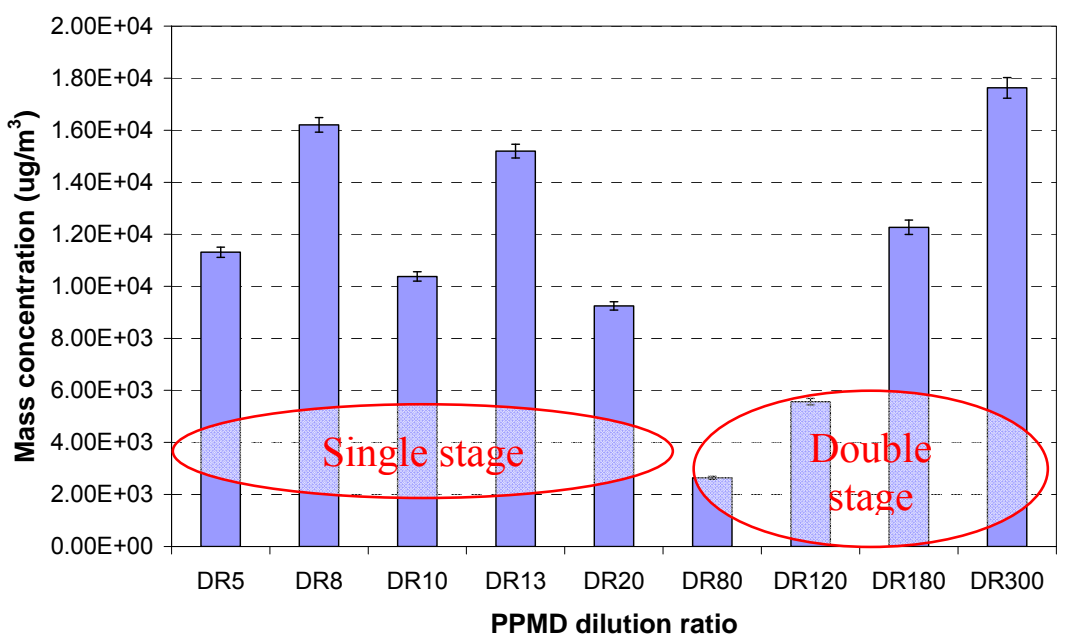

Figure 85 Particle mass concentration with PPMD dilution (122 Nm @1800 rpm) 
When the engine was running at $102 \mathrm{Nm}$, the total number concentration showed a growing trend with increased dilution ratios in both single and dual dilution stage modes. However, the particle number concentration levels in double dilution stage mode were lower than the level in single dilution mode. The mass concentration levels when the engine was running at $102 \mathrm{Nm}$ showed an average value of $3,400 \mu \mathrm{g} / \mathrm{m}^{3}$ and a standard deviation of $800 \mu \mathrm{g} / \mathrm{m}^{3}$. When the engine was running at $122 \mathrm{Nm}$, the total number concentration levels showed a declining trend with increased dilution ratios in single dilution mode and growing trend in dual dilution stage mode, and the lowest number concentration level occurred at dilution ratio equaled to 80 in double dilution mode. The mass concentration levels when the engine was running at $122 \mathrm{Nm}$ were in a decreaing or increasing pattern in single dilution mode and increased with larger dilution ratio values in double dilution mode.

The measurement results from the PPMD dilution showed more complex patterns than the results of other two methods. The change of particle size distribution curves, number and mass concentration levels were not as strongly correlated to the dilution ratios as other two methods indicated. This was largely due to effects of the sophisticated dilution strategy of the MPS diluter and the complexity of the diluter plumbing. As shown in Figure 8, even though each one MPS diluter is regarded as a one-stage diluter, it is actually composed of two mixing chambers. The minor flow rate and major flow rate were calculated in real time and generated to create appropriate vacuum pressures for sucking sample flow through one laminar flow capillary and one transport tube. To keep sampling at a constant dilution ratio, the sample flow rate and total dilution flow rate were maintain as constant value while the major and minor flow rates were adjusted in real time by monitoring all flow rates and the exhaust flow temperature and pressure. The dilution ratios of the sample flow in two mixing chambers changed when the exhaust temperature and pressure changed, even the total dilution ratio and total dilution flow were maintained as constants. Without further knowledge of how the primary and secondary mixing dilution's effects on the particle size distributions, it is difficult to access the total dilution ratio effects. When the double MPS diluters were used for sampling, the sample flow were actually diluted four times and the sample flow went 
through valves and more plumbing to reach the DMS500 sample probe, which made the analysis of dilution effects more difficult. For these reasons, the details of the DPM evolution inside the PPMD were not analyzed in depth.

Since there are different partial dilution devices in existence and they work on different dilution and control strategies, the measurement results from the PPMD may lack the generality of dilution effects of partial dilution devices. The PPMD measurement results should be regarded as device-dependent and to serve as an example of partial flow device's dilution effects on the particle size distributions.

\subsection{Post-tailpipe Plume Test}

\subsubsection{Dilution Ratios}

Dilution ratios at the exhaust plume post-tailpipe centerline were measured up to $5 \mathrm{~m}$ (16 feet) from the engine exhaust when engine was operated at $102 \mathrm{Nm}$ and $122 \mathrm{Nm}$ at $1800 \mathrm{rpm}$. The accuracy of dilution ratio measurement was critical to the stack equivalently corrected particle size distributions. The measurement results indicated the $\mathrm{NO}_{\mathrm{x}}$ emissions were undetectable levels beyond about $2.3 \mathrm{~m}$ (7.5 feet) post-tailpipe. The measured dilution ratios up to $2.3 \mathrm{~m}$ post-tailpipe are shown in Figure 86. Further analysis of measured particle size distributions revealed that stable particle size distributions could be only obtained for locations with dilution ratios under 35 . The dilution ratios associated with measured particle size distributions were mostly of interest the tailpipe test, and they were not clearly shown in Figure 86. Figure 87 showes the zoomed in view of partial curve in Figure 86 with dilution ratios under 40 to provide a clearer view of dilution ratios within short distance post-tailpipe.

Figure 86 and Figure 87 show that at locations within $150 \mathrm{~cm}$ post-tailpipe centerline, the corresponding dilution ratios almost linearly increased with the distance with small slopes. With distance increase after $150 \mathrm{~cm}$, the dilution ratios increased sharply. The measured dilution ratios when the engine was running at $122 \mathrm{Nm}$ were consistently lower than those measured at $102 \mathrm{Nm}$ at same sampling locations. It could be explained that when the engine exhaust was hotter and with a higher emitting velocity, 
the raw exhaust traveled further post-tailpipe and was mixed with fresh air less completely, correspondingly, smaller dilution ratio values.

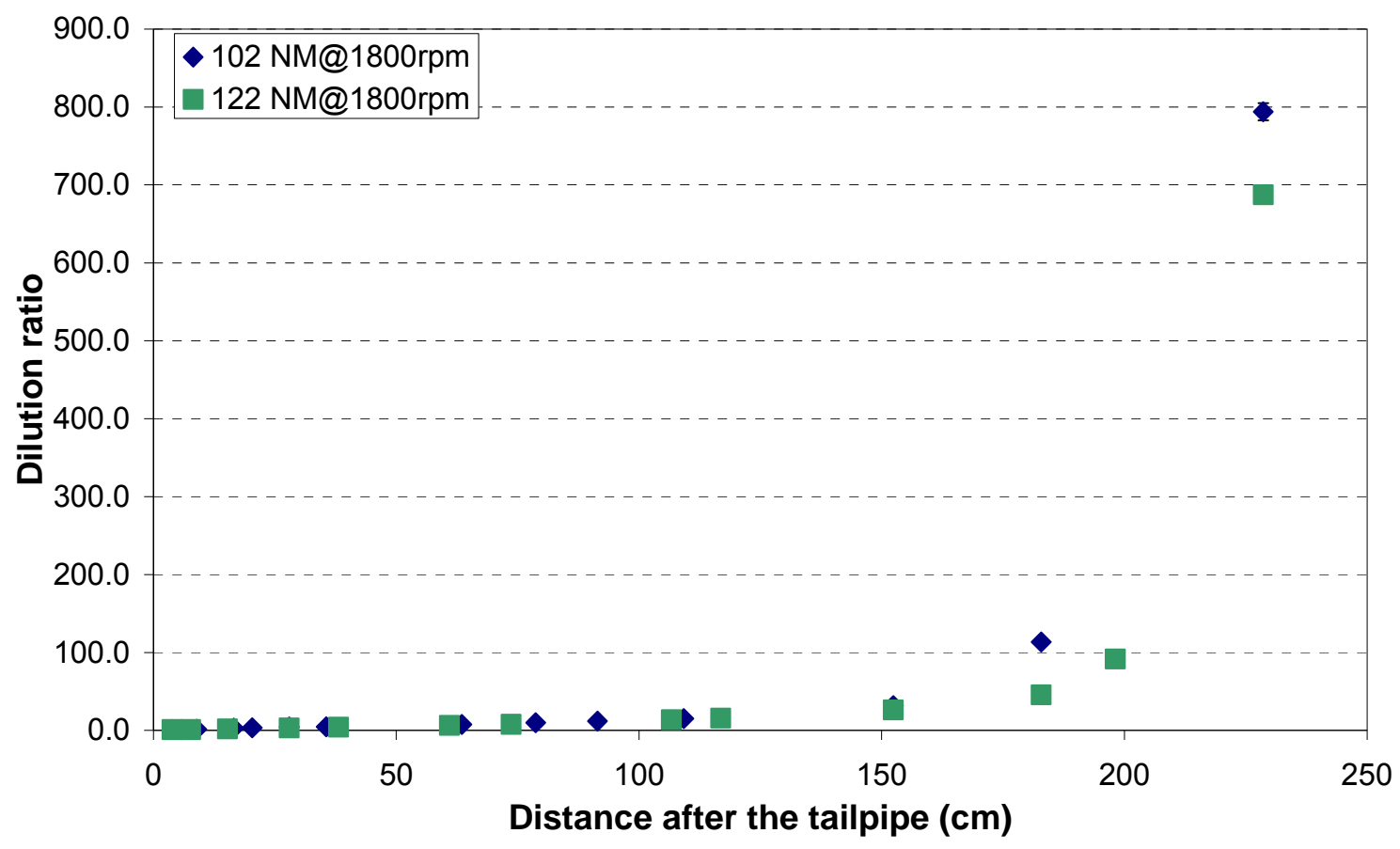

Figure 86 Dilution ratios of exhaust plume post-tailpipe centerline

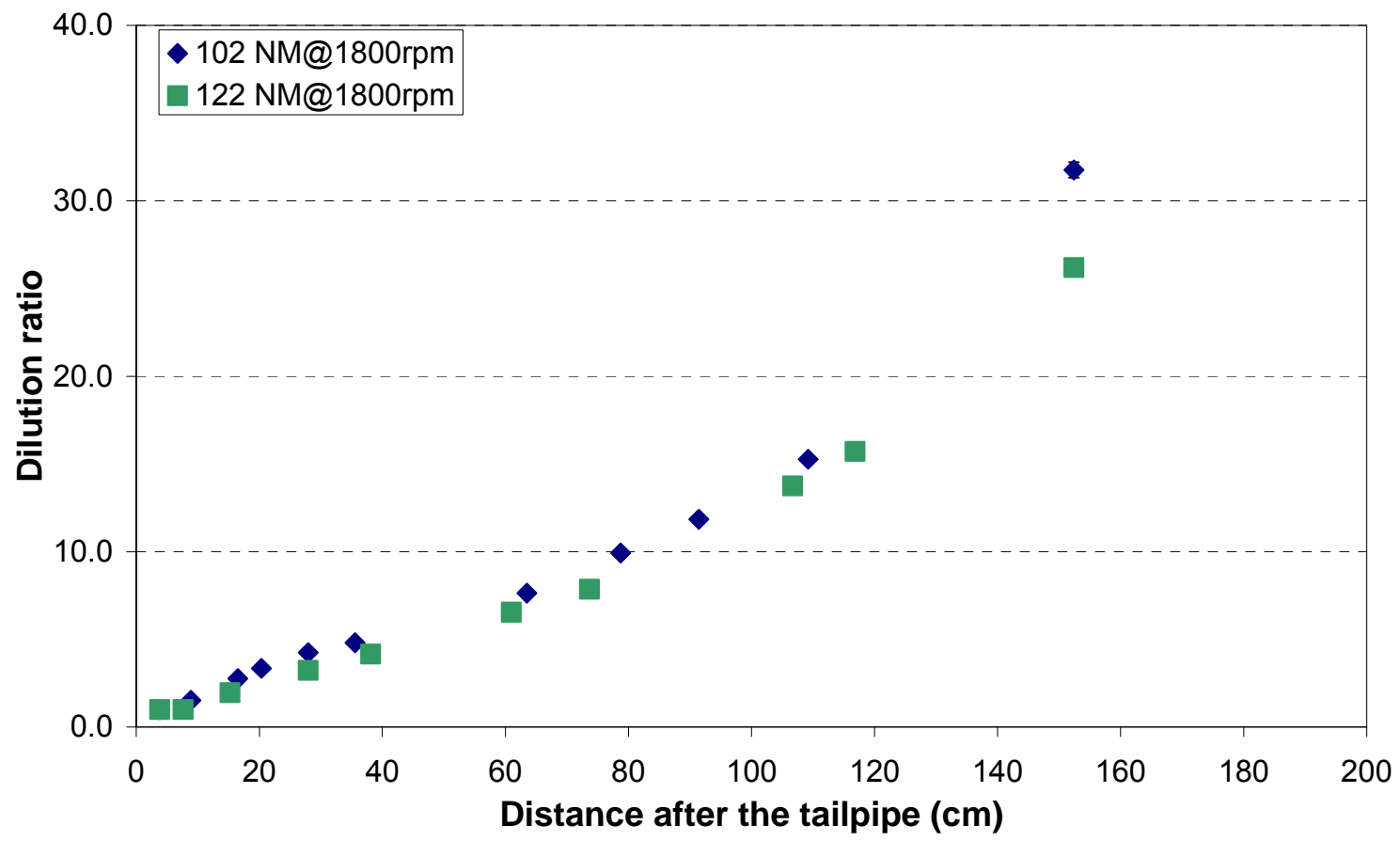

Figure 87 Dilution ratios of exhaust plume post-tailpipe centerline zoomed view 


\subsubsection{CFD Analysis of Exhaust Plume}

The measured dilution ratios after the exhaust tailpipe centerline provided information needed for measured particle size distributions to be back corrected to engine stack equivalent values. However, the measured dilution ratios were only performed after a short distance along the post-tailpipe centerline and hence only unveiled a very limited profile of the exhaust plume dispersion. In order to obtain more information about the exhaust plume dispersion and have more ideas about the role of measure dilution ratio profile in the whole exhaust plume, a CFD analysis of the exhaust plume was conducted. Furthermore, the results of CFD analysis could provide information on the residence times which could not be obtained from dilution ratio measurement. The measured dilution ratios at post-tailpipe center line were compared to the simulation results for the purpose of model verification.

The commercially available CFD software, Fluent was used to perform the numerical analysis for the exhaust plume dispersion simulation. In order to increase the simulation accuracy, reduce the computational time of the numerical simulation, and overcome the limitation of available computer memory, the computational domain of the CFD analysis was created and separated into two sub-domains and the CFD analysis was

performed in two steps. The computational grids generated for the two sub-domains are shown in Figure 88.

A stand-alone simulation was performed first using domain 2 (see Figure 88) to generate the velocity boundary layer profile for inlet shown in domain 1 . The inlet of domain 2 was assigned with a uniform velocity inlet profile normal to the inlet surface. The side wall was $7.6 \mathrm{~m}$ wide and $2.0 \mathrm{~m}$ high and the ground wall was set as being $7.6 \mathrm{~m}$ by $3.8 \mathrm{~m}$ in domain 2 . The surfaces including the outlet surface other than inlet and wall in domain 2 were set to open to atmosphere.

Once the velocity profile was obtained by simulation in domain 2 , the profile was regarded as the wind boundary layer profile and was applied as the velocity profile to the inlet of domain 1. A side surface of $3 \mathrm{~m}$ wide and $2 \mathrm{~m}$ high and a ground surface of $3.8 \mathrm{~m}$ 
by $3 \mathrm{~m}$ were set in domain 1 . Both surfaces were modeled as no-slip walls as walls in domain 2. A mass flow inlet boundary condition was assigned to the tailpipe in domain 1 to model the exhaust being emitted. The tailpipe was modeled as $5 \mathrm{~cm}$ long with $6.7 \mathrm{~cm}$ in diameter and stuck out from the middle of the side wall at $0.7 \mathrm{~m}$ high in domain 1 . This gives a better visual view of the tailpipe location, the meshing of the cross section passing the tailpipe centerline and normal to the ground and the side wall in shown in Figure 89. The meshing shown in Figure 89 had been numerically adapted to have denser meshing in areas with higher $\mathrm{NO}_{\mathrm{x}}$ concentration level gradients.
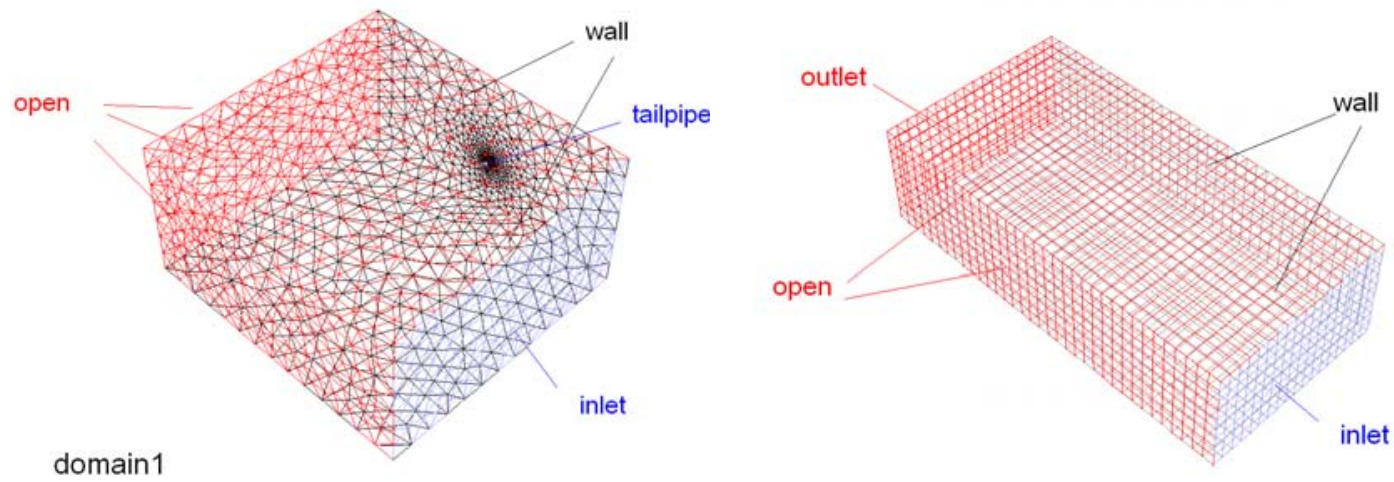

domain 2

\section{Figure 88 CFD grids of computation domains}

The real world's terrain, building effects on the wind boundary layer profiles over the ground and side wall could be much more complex than those effects in the computational domains shown in Figure 88. Furthermore, the wind velocity and direction were difficult to monitor over the test field in real time over a time scale of seconds or minutes. A constant value normal to the boundary was assumed and applied to the wind inlet boundary condition in the CFD simulation. Since the CFD model was largely simplified compared to the real world dilution condition, the purpose of the CFD analysis was to provide auxiliary information of exhaust plume dispersion and to predict exhaust flow patterns post-tailpipe with a reasonable accuracy. 


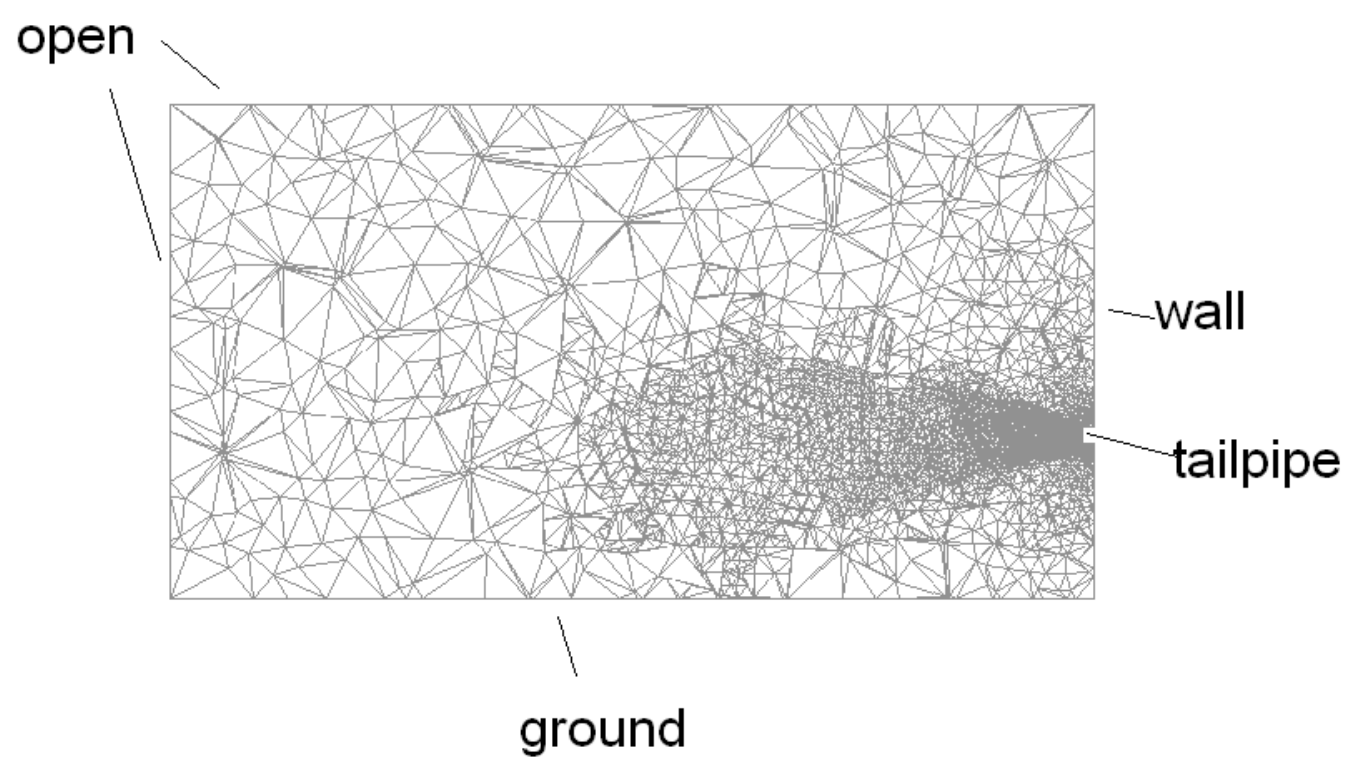

Figure 89 Meshing of the tailpipe centerline cross section

The CFD models for the engine running at $102 \mathrm{Nm}$ and $122 \mathrm{Nm}$ were identical with only a difference in exhaust temperature. The $\mathrm{k}-\varepsilon$ viscous model with standard wall functions and species transport were applied in the CFD models. The exhaust flow was set to have a mass flow rate of $0.037 \mathrm{~kg} / \mathrm{s}$ with a temperature of $600 \mathrm{~K}$ at $102 \mathrm{Nm}$ and $680 \mathrm{~K}$ at $122 \mathrm{Nm}$. These values were representative of flow and temperature of the engine at this load. The ambient temperature was set to be $278 \mathrm{~K}$ with 1 atm ambient pressure. The initial simulated wind velocity was set to be $0.44 \mathrm{~m} / \mathrm{s}(1 \mathrm{mph})$ and normal to the inlet in computational domain 2. The analysis of CFD models was focused on this wind velocity setting. To give a example view of the exhaust plume dispersion simulation results, Figure 90 shows the streamlines of exhaust and side wind flow color with temperature.

The exhaust plume dilution ratios were assumed to be measured along the posttailpipe centerline. However, the offsets from the centerline for the sample probe tip during the sampling process were taken into consideration when the measured dilution ratios were compared with CFD simulation results. The possible offset areas from the centerline were estimated to be a rectangle and linearly increased from 0 to $5 \mathrm{~cm}$ in size with post-tailpipe distance from 0 to $2 \mathrm{~m}$. The simulated centerline dilution ratios were 
compared to the measurement values with information obtained from dilution ratio values of eight offset points. To illustrate the locations of selected points for simulated dilution ratios in the offset area, an exaggerated view of the offset area with dilution ratio recording point for CFD simulation is shown in Figure 91.

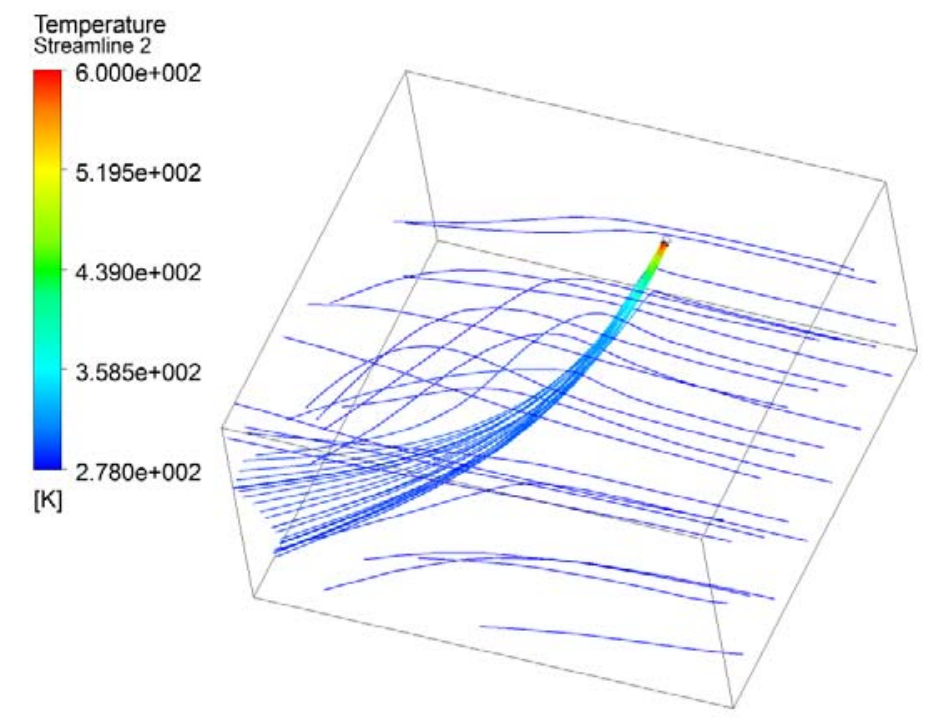

Figure 90 Streamline of simulated plume dispersion (running at $102 \mathrm{Nm}$ with 0.44 $\mathbf{m} / \mathbf{s}$ wind speed)

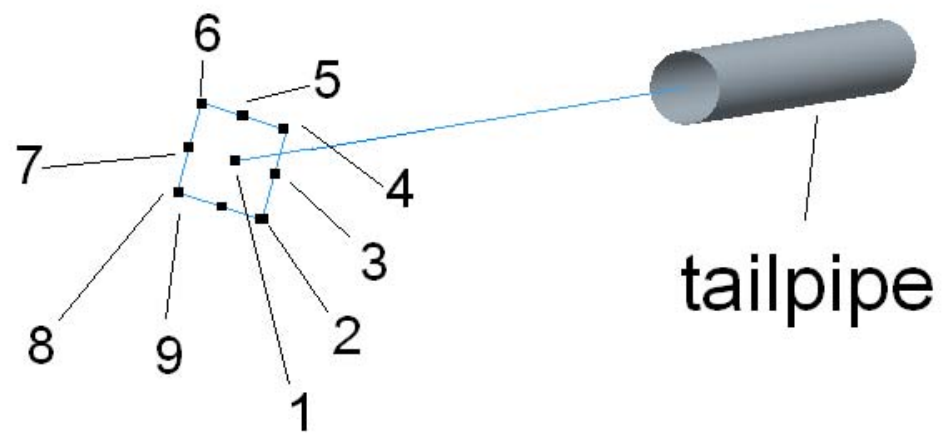

\section{Figure 91 CFD simulation result recording points in an offset area}

There were no strict rules for selection of offset area shape and recording point locations for simulation and measurement comparison. The idea of introducing offset areas and more recording points was to give more information on dilution ratio variation caused by small changes of sampling locations. The comparisons of measured and simulated dilution ratios post-tailpipe centerline are shown in Figure 92 and Figure 93. 
The upper and lower bound the error bars of the simulated dilutions ratios corresponding to the maximum and minimum dilution ratio values of the nine point values on the offset area as shown in Figure 91.

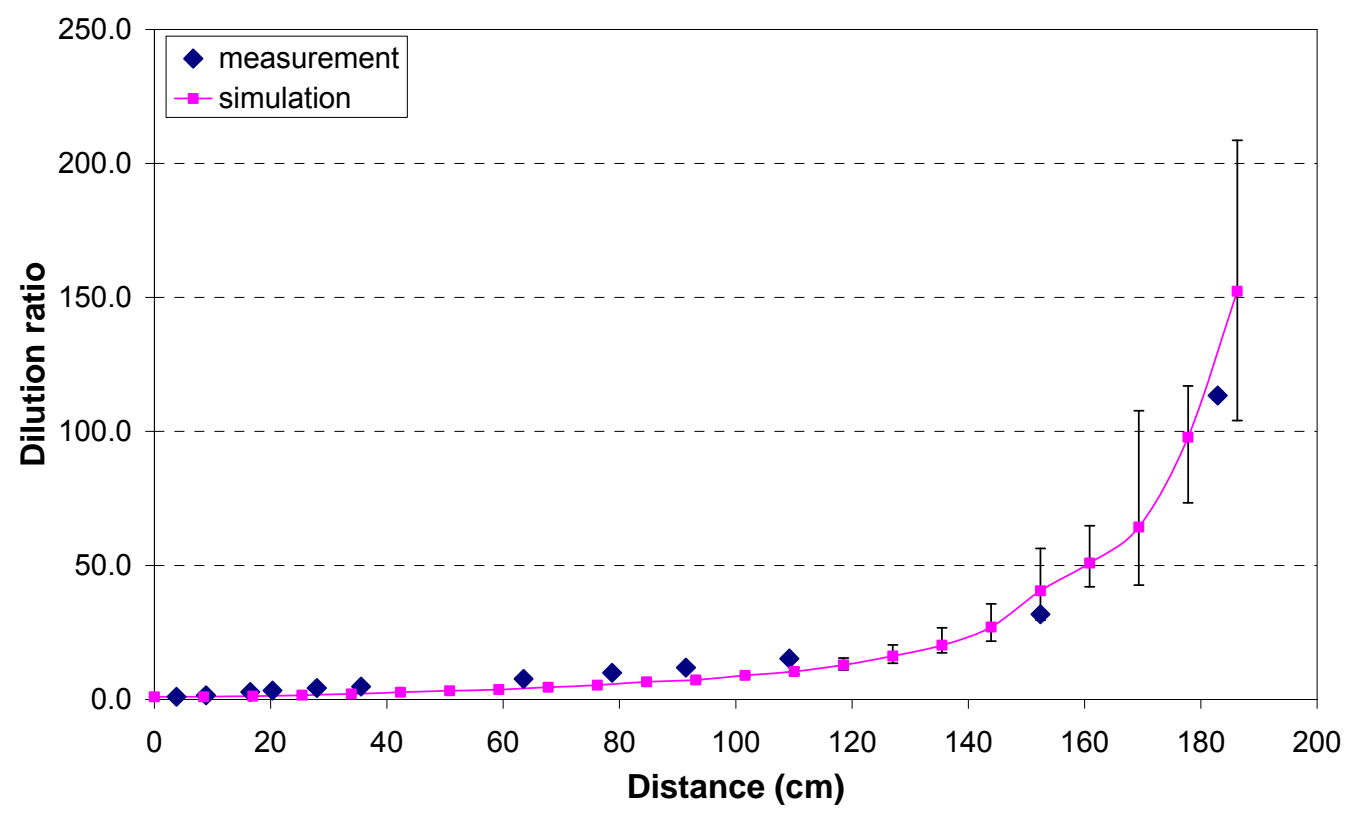

Figure 92 Measured and simulated dilution ratios post-tailpipe centerline (102 Nm @ 1800 rpm)

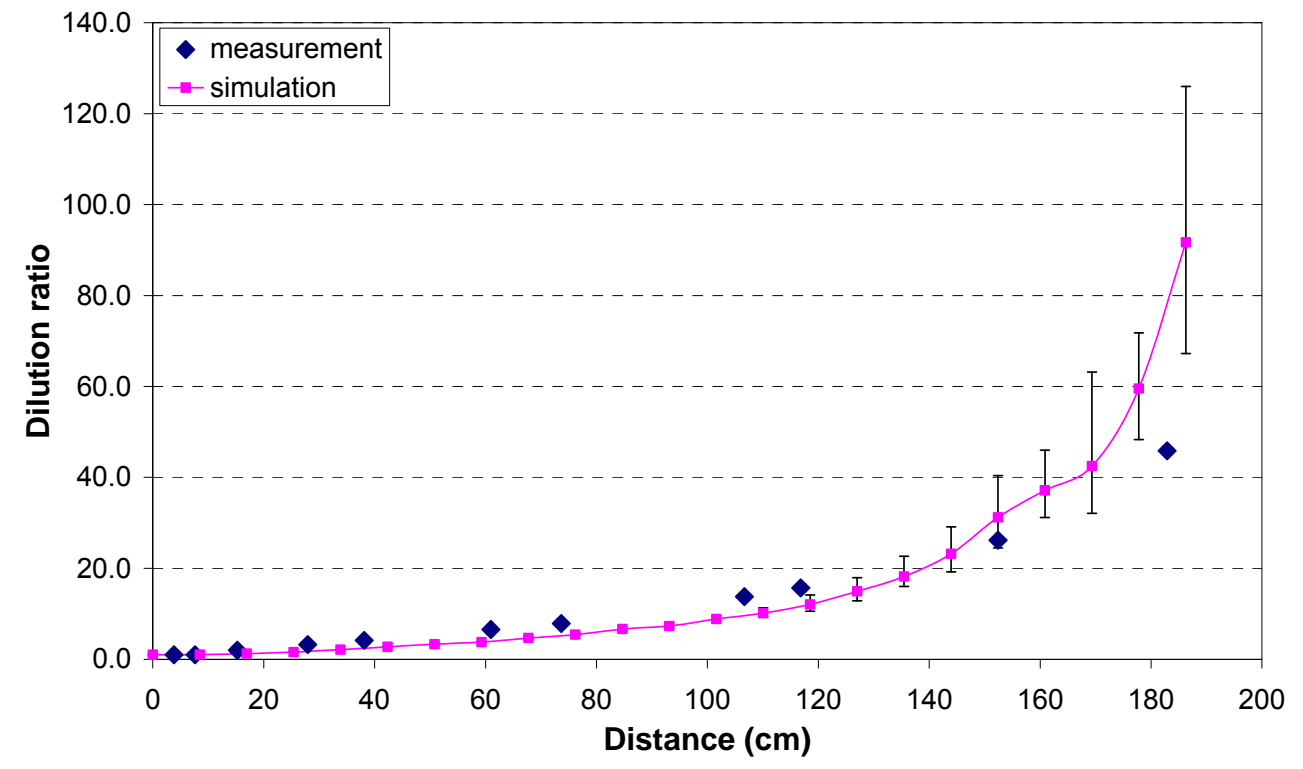

Figure 93 Measured and simulated dilution ratios post-tailpipe centerline (122 Nm @ 1800 rpm) 
It can be seen from Figure 92 and Figure 93 that the CFD simulated dilution ratios for the exhaust plume dispersion post-tailpipe centerline could provide reasonable accuracy compared with measured dilution ratio values. Both figures also indicate that the differences of dilution ratios within a small offset area could be very high with high centerline dilution ratio values. The CFD analysis of the modeling results on other variables should be able to provide auxiliary information for post-tailpipe dilution process in the following section of particle size distribution analysis. Important information that can be estimated from the CFD analysis is the effects of wind speed on the centerline dilution ratios. Figure 94 shows the simulated centerline dilution ratios for wind speeds from calm to $5 \mathrm{mph}(2.2 \mathrm{~m} / \mathrm{s})$ with the engine running at $102 \mathrm{Nm} @ 1800 \mathrm{rpm}$.

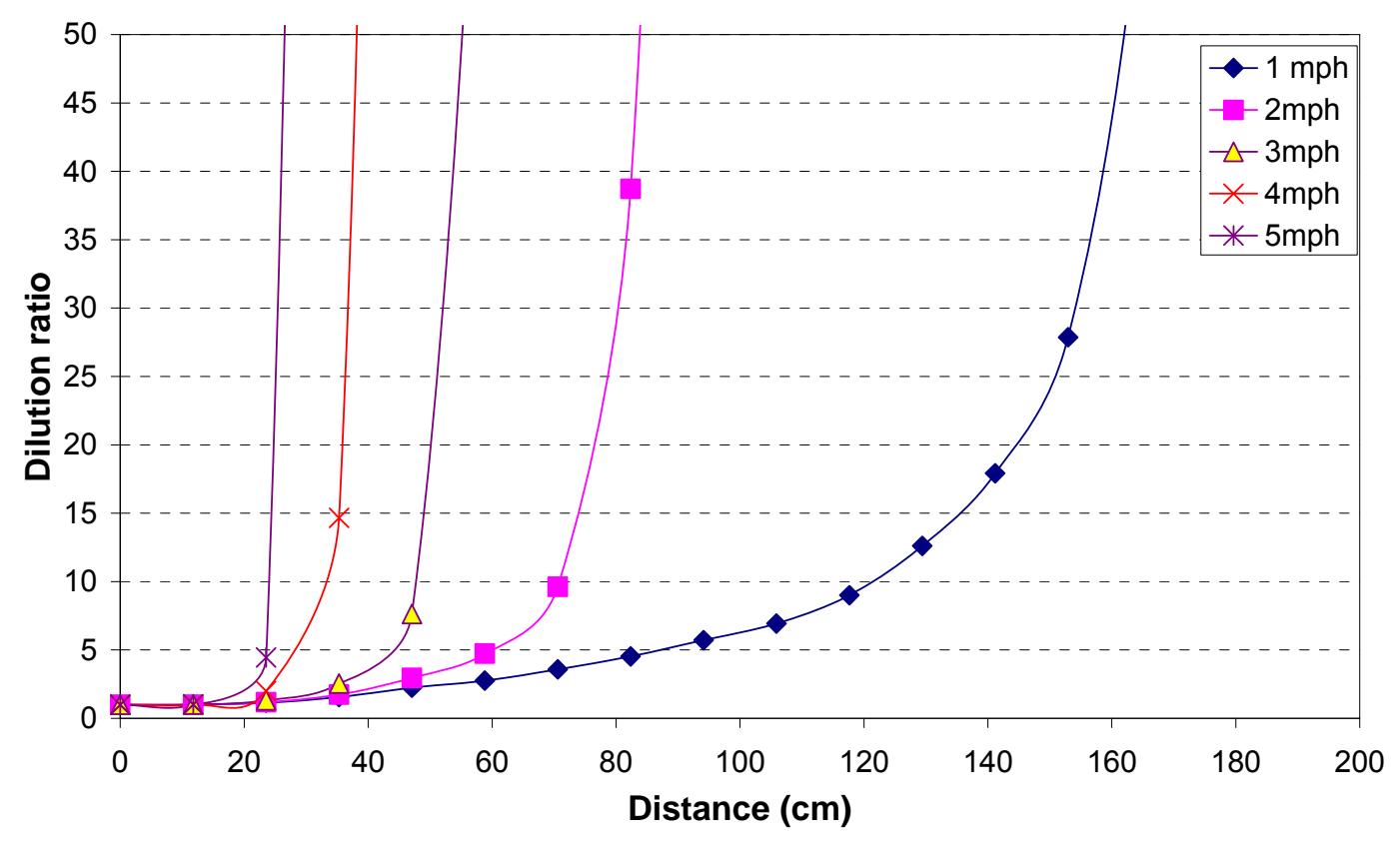

Figure 94 CFD simulated centerline dilution ratios with different wind speeds (102 Nm@1800 rpm)

Dilution ratios shown in Figure 94 indicate the wind speeds had large effects on the post-tailpipe exhaust plume dilution. If $\mathrm{NO}_{\mathrm{x}}$ emission concentration levels were measured in field with corresponding wind speeds to determine the dilution ratios, when the wind speed was increased from $1 \mathrm{mph}$ to $5 \mathrm{mph}$, the post-tailpipe distance with detectable $\mathrm{NO}_{\mathrm{x}}$ emission concentration levels would be significantly reduced from above $1.6 \mathrm{~m}$ to around $0.2 \mathrm{~m}$. The results of centerline dilution ratio simulation revealed the difficulty of measuring post-tailpipe exhaust plume dilution ratios even under the light 
breeze wind conditions with the wind speed less than $1 \mathrm{mph}$ because of the innate effect of the wind.

\subsubsection{Particle Size Distribution}

Stable particle size distributions for the engine running at $102 \mathrm{Nm}, 1800 \mathrm{rpm}$ and at $122 \mathrm{Nm}, 1800 \mathrm{rpm}$ were measured post-tailpipe centerline up to $152 \mathrm{~cm}$ (60 inches) and shown in Figure 95 and Figure 96. Particle size distributions measured beyond this threshold distance started to display random oscillation patterns and they were not presented.

Particle size distributions shown in Figure 95 and Figure 96 display similar shapes change trends with increasing dilution ratio values. The peaks of particle size distribution curves for the engine running at $102 \mathrm{Nm}$ and $122 \mathrm{Nm}$ increased with higher dilution ratios, and with slight peak shifting to the smaller diameter end. For engine running at $102 \mathrm{Nm}$, the peak of particle size distribution curves shifted from $23.7 \mathrm{~nm}$ to $15.7 \mathrm{~nm}$. For engine running at $122 \mathrm{Nm}$, the peak of particle size distribution curves shifted from $23.7 \mathrm{~nm}$ to $17.8 \mathrm{~nm}$. A clear curve peak shifting for particle size distribution at dilution ratio 4.2 to particle size distribution at dilution ratio 6.5 was observed in Figure 96. From the earlier discussion on the particle transport and transformation during the dilution process in the tunnel test section, Figure 95 and Figure 96 indicate that high dilution ratios were in favor of nano-particle nucleation and such a nucleation process was stronger for particles in smaller diameter size range with higher dilution ratios, as being illustrated by the particle size distribution curves' peak shifting. Along with the change of particle size distribution curve shapes, particle number and mass concentration levels also

provided information related to particle processes during exhaust dilution. The stack corrected particle number and mass concentration levels are shown in Figure 97, Figure 98, Figure 99, and Figure 100. 


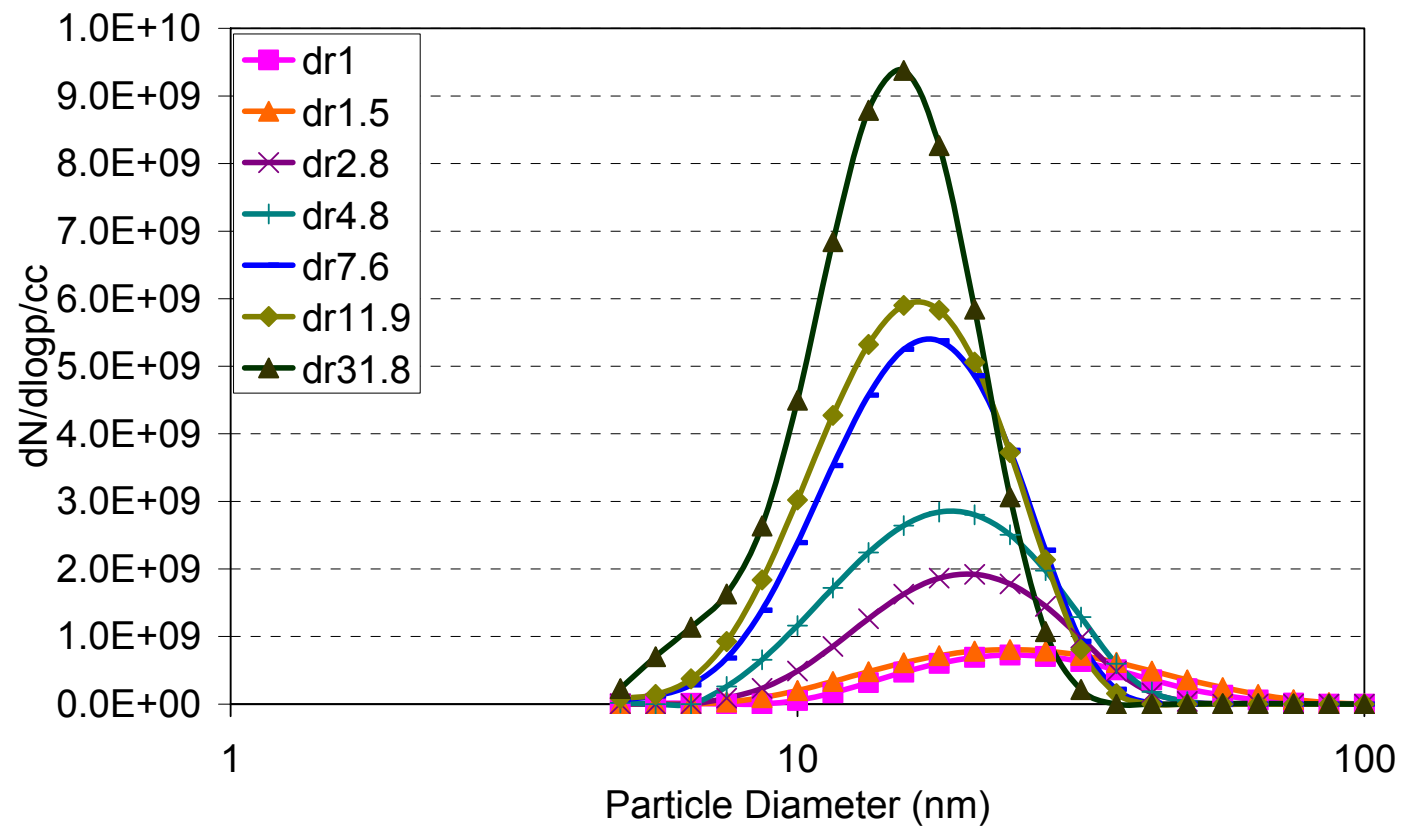

Figure 95 Measured particle size distributions post-tailpipe centerline (102 Nm @ 1800 rpm)

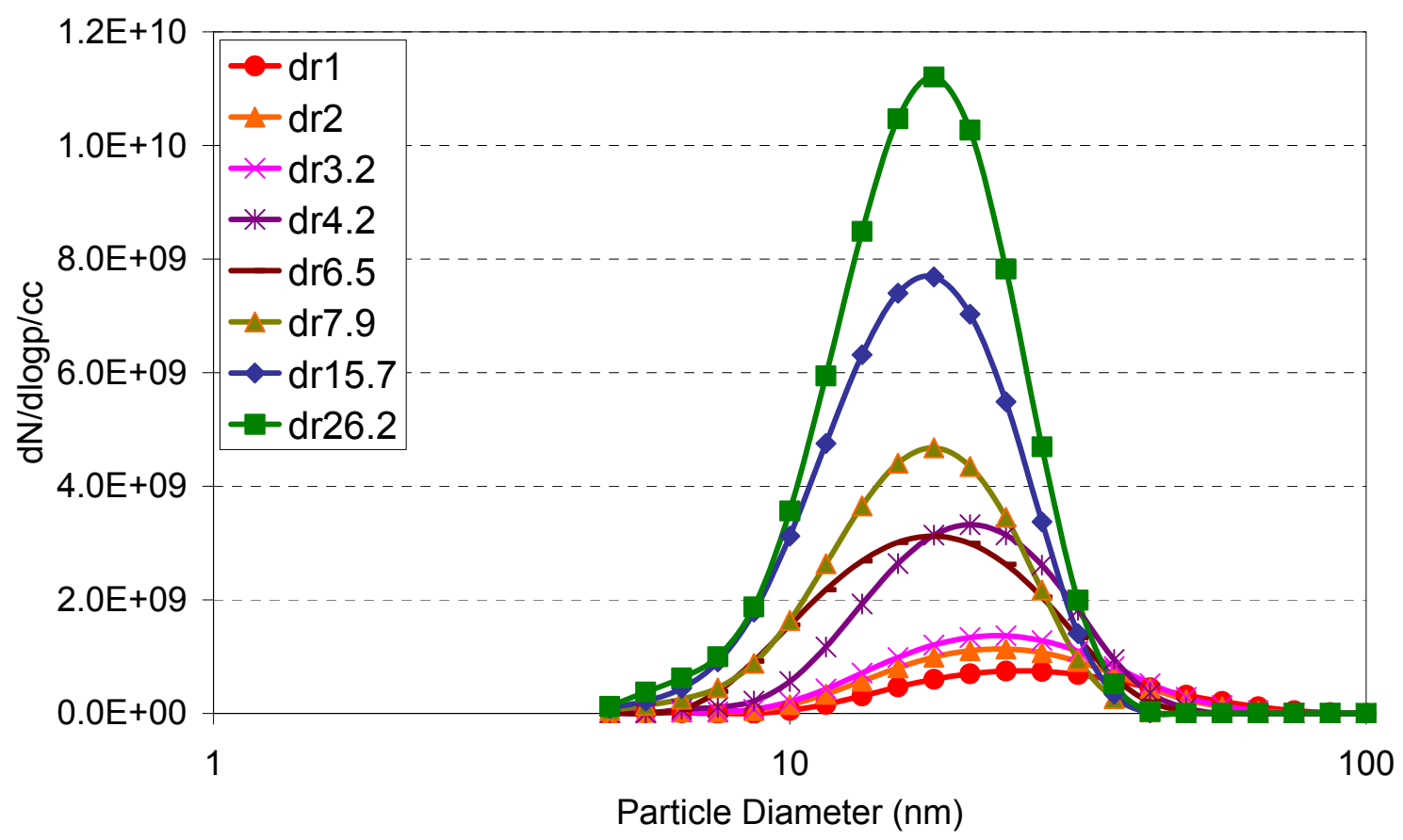

Figure 96 Measured particle size distributions post-tailpipe centerline (122 Nm @ 1800 rpm) 


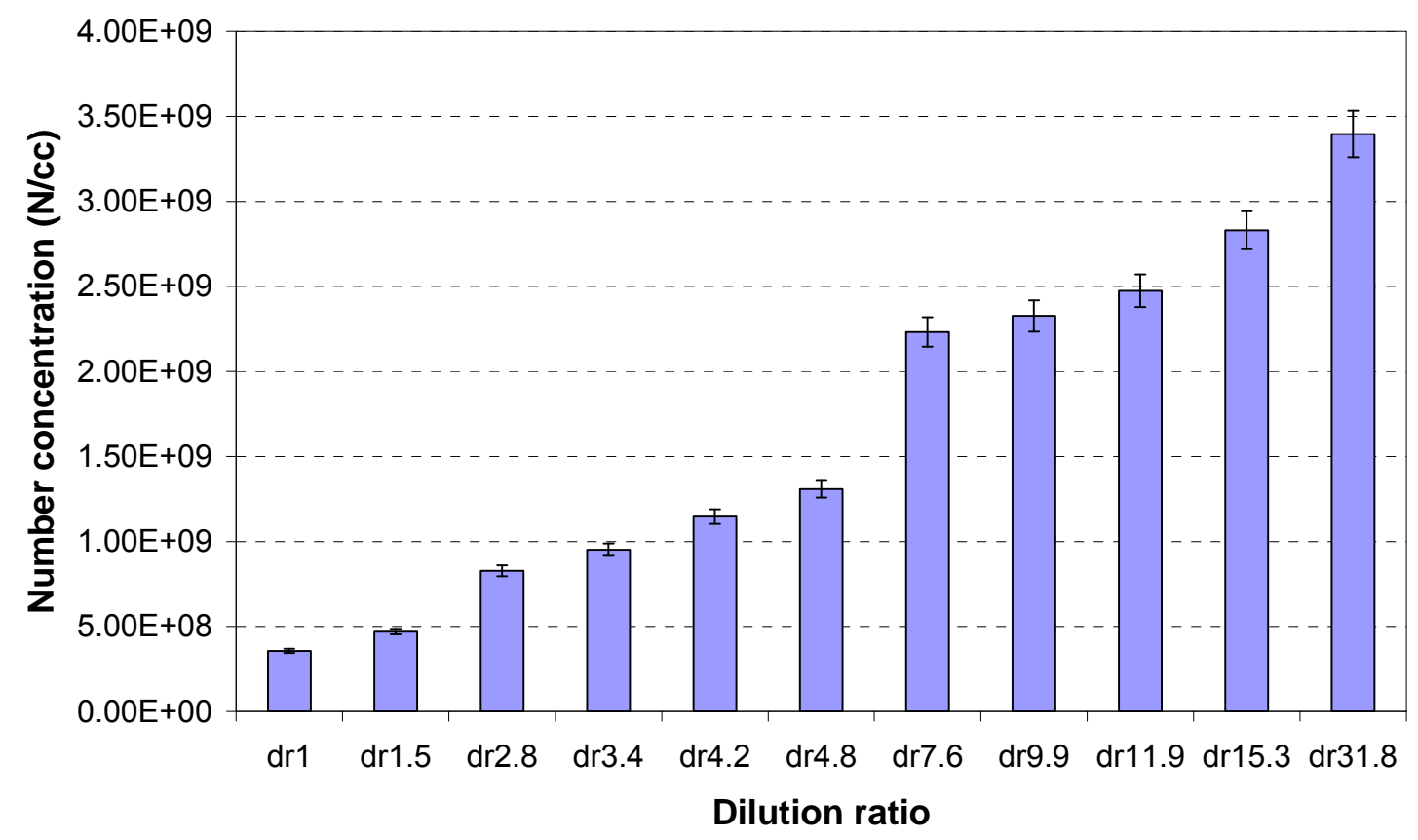

Figure 97 Particle number concentration post-tailpipe centerline (102 Nm @ 1800 rpm)

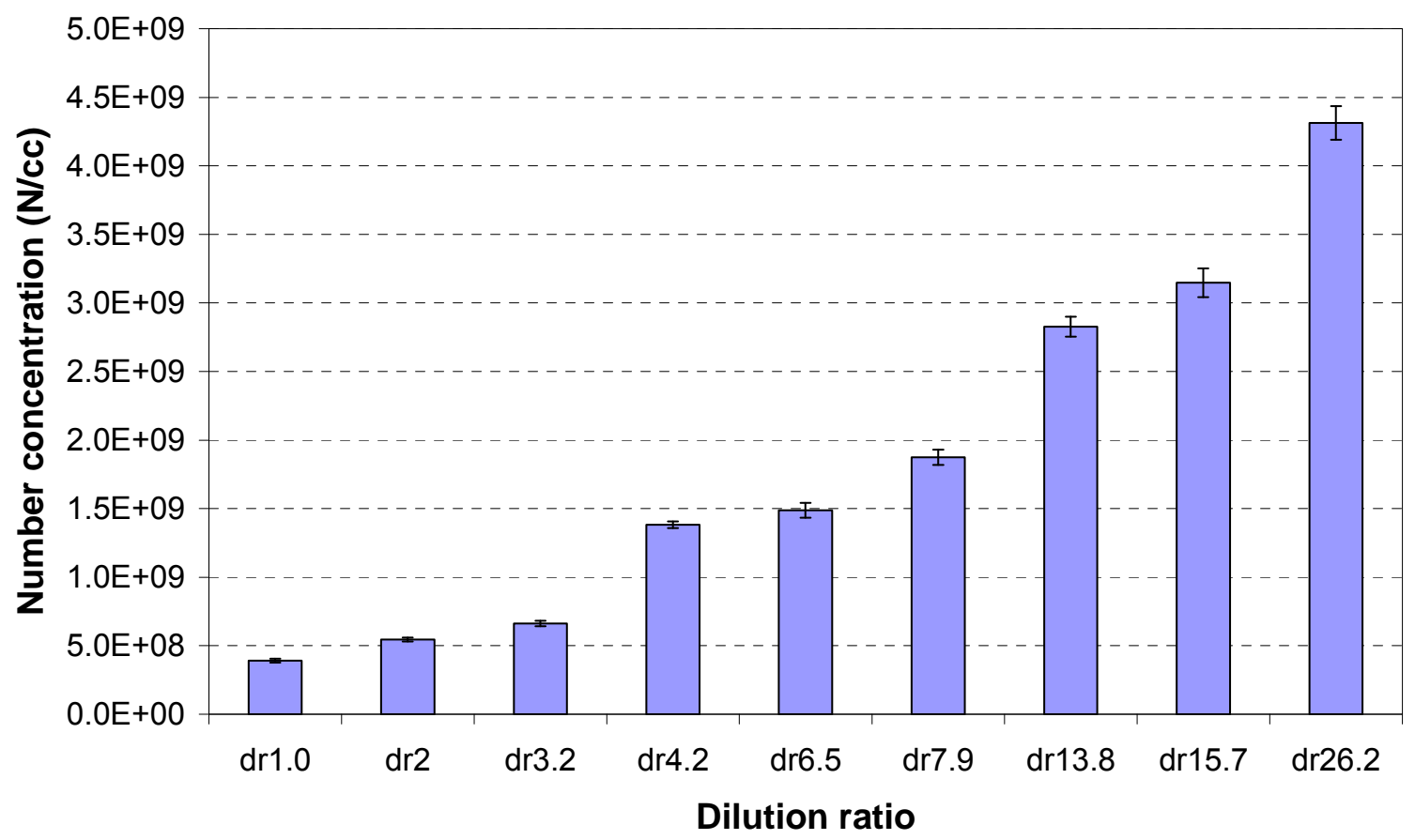

Figure 98 Particle number concentration post-tailpipe centerline (122 Nm @ 1800 rpm) 
As shown in Figure 97 and Figure 98, particle number concentration levels increased along the post-tailpipe centerline, with increasing dilution ratios. It reinforces the inference that the nucleation was the dominant process when the exhaust plume was diluted along the post-tailpipe centerline. As discussed earlier, the nucleation process increases the total particle count and mass. However, the increase of particle mass caused by the nucleation process might be negligible compared to increased particle number count due to the small mass fraction of nano size particle in the whole particle size distribution range. There was no observable increase in mass with distance from centerline.

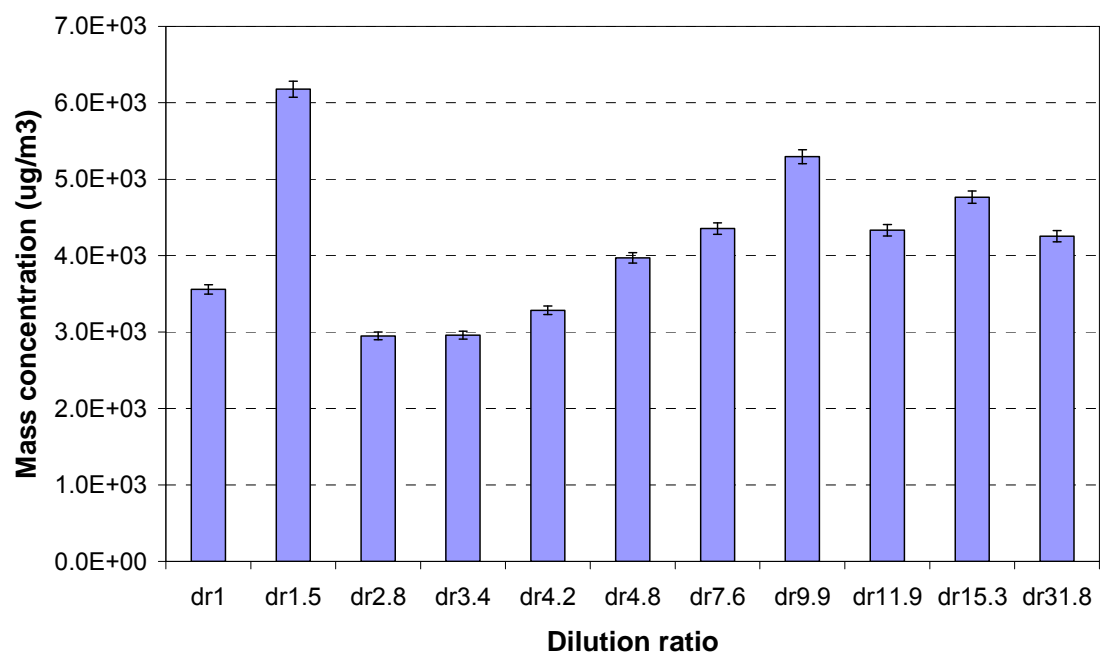

Figure 99 Particle mass concentration post-tailpipe centerline (102 Nm @ 1800 rpm)

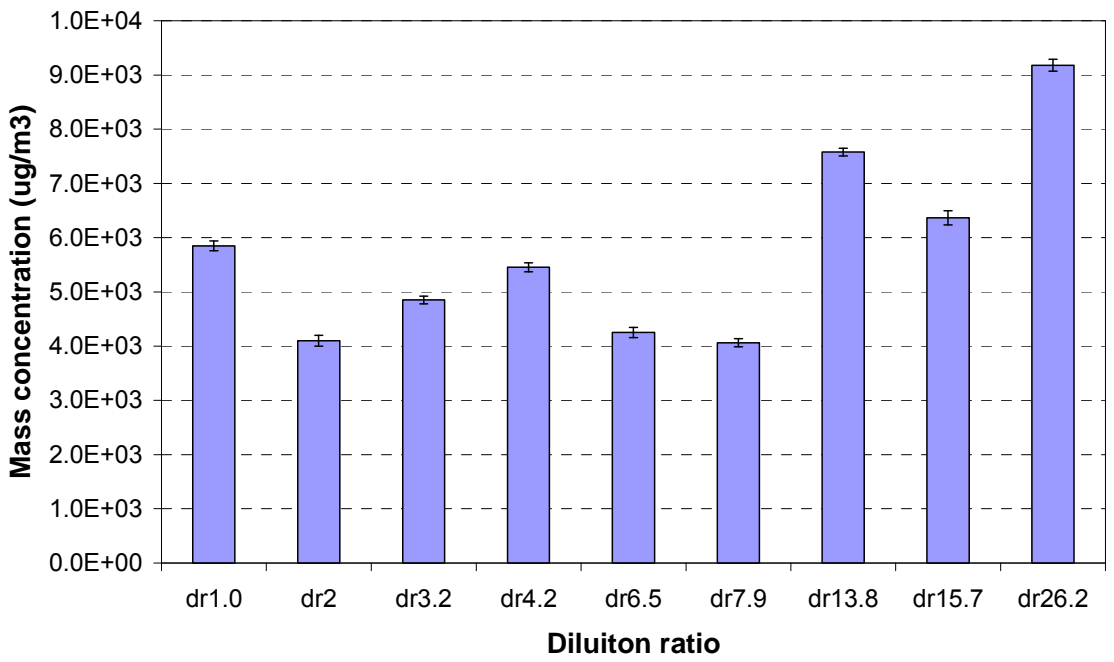

Figure 100 Particle mass concentration post-tailpipe centerline (122 Nm @ 1800 rpm) 
Particle mass concentration levels shown in Figure 99 and Figure 100 show more arbitrary patterns relating the dilution ratios compared the number concentration levels shown in Figure 97 and Figure 98. The discussion in the tunnel test stated processes might influence the particle mass concentration included nucleation (increase the number count and mass), adsorption (no effect on the number count and increase the mass), coagulation (no effect on the mass and decrease the number count), SOF evaporation (decrease the number count and mass), and diffusion loss to the sample probe.

It has been confirmed from the particle size distribution curves and the number count bar graphs that the nucleation was the dominant process during the dilution. Since the nucleation and adsorption were considered as two competing processes, the adsorption process was regarded to be largely suppressed by the dominant nucleation process. Since the coagulation process has no effect on the total particle mass, the variations of particle mass during the exhaust plume dilution then were mainly due to the strength of SOF evaporation and diffusion loss to the sample probe inner wall.

\subsection{Comparison of Dilution Methods}

\subsubsection{Same Dilution Ratio}

\section{a. Tunnel vs. Post-tailpipe Test}

The particle size distribution curve shapes were first compared at the same dilution ratio (dilution ratio values within $2.5 \%$ of each other were considered to be same in this work) for different dilution methods. 175 measured stack corrected particle size distributions were compared to each other and they are summarized in Table A2 with each particle size distribution labeled with number in order.

Figure 101, Figure 102 and Figure 103 show the stack equivalent particle size distribution curves of tunnel and post-tailpipe tests at dilution ratios 14, 16, and 26 when the engine was running at $122 \mathrm{Nm}$. 


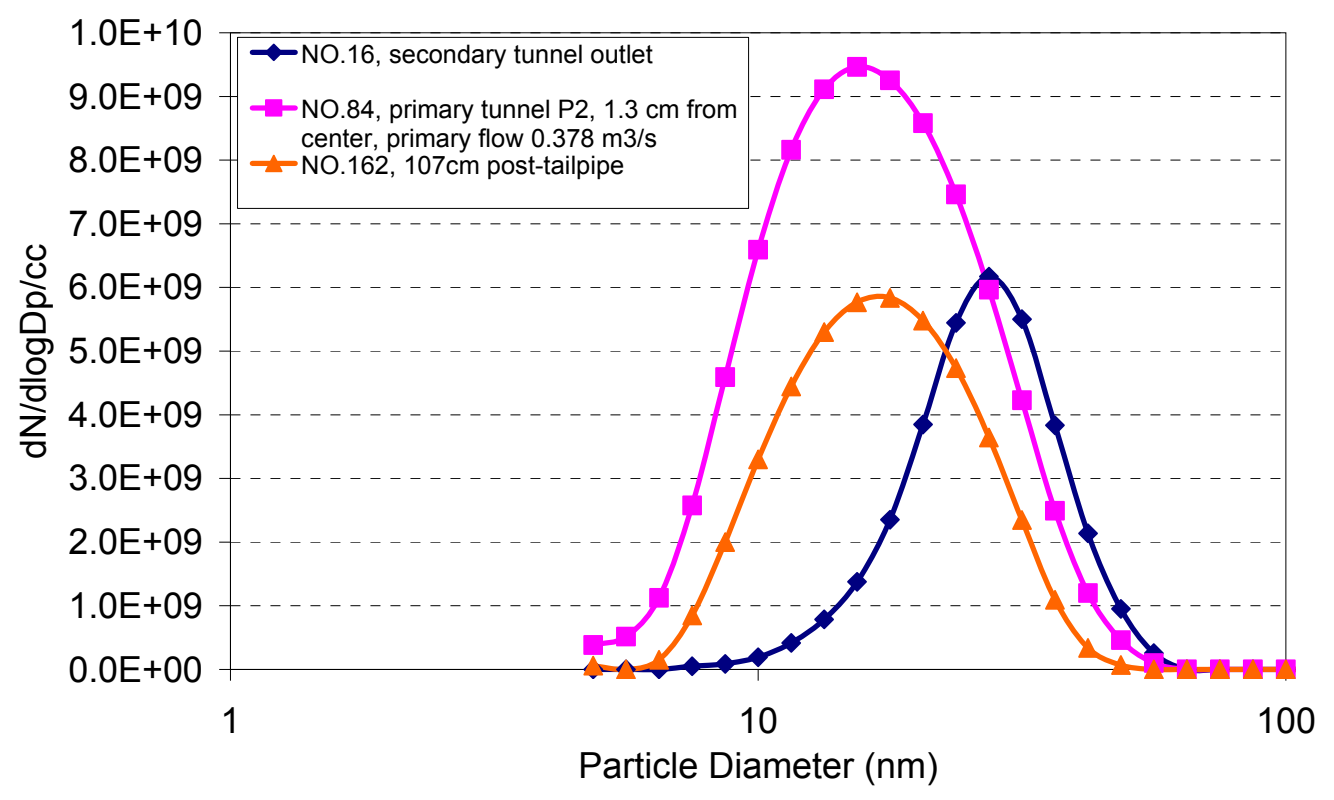

Figure 101 Particle size distributions of tunnel and post-tailpipe test at dilution ratios of 14 (122 Nm @ 1800 rpm)

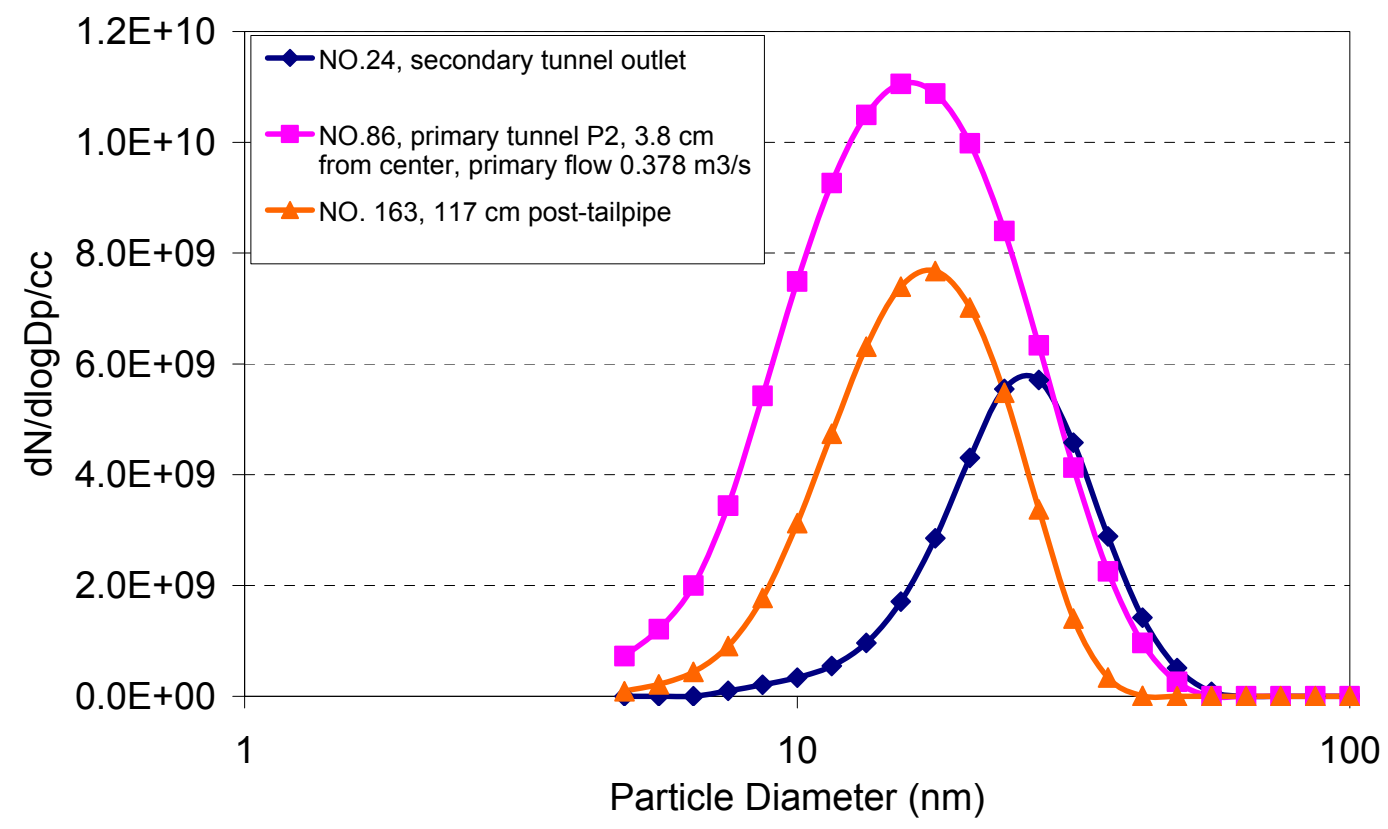

Figure 102 Particle size distributions of tunnel and post-tailpipe test at dilution ratios of $16(122 \mathrm{Nm} @ 1800 \mathrm{rpm})$ 


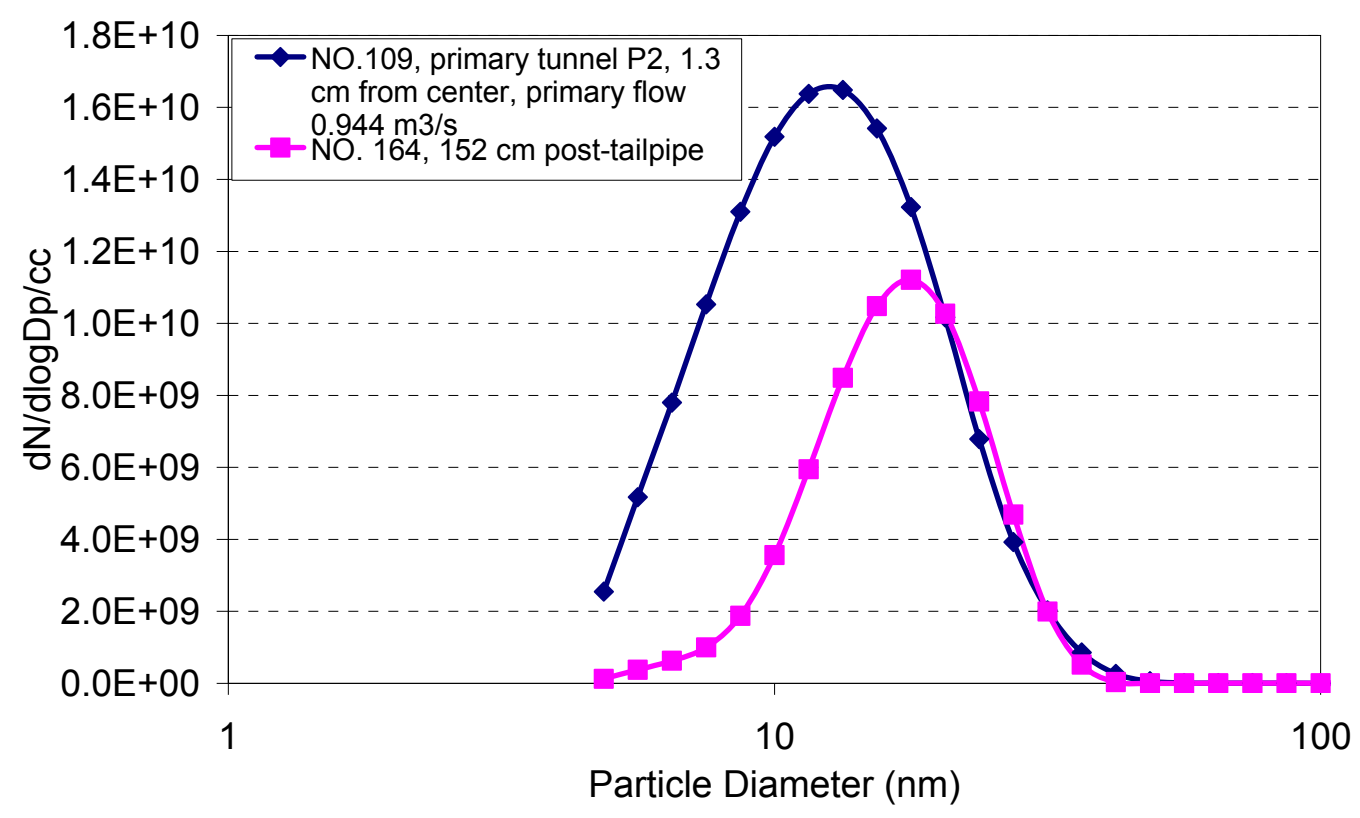

Figure 103 Particle size distributions of tunnel and post-tailpipe test at dilution ratios of 26 (122 Nm @ 1800 rpm)

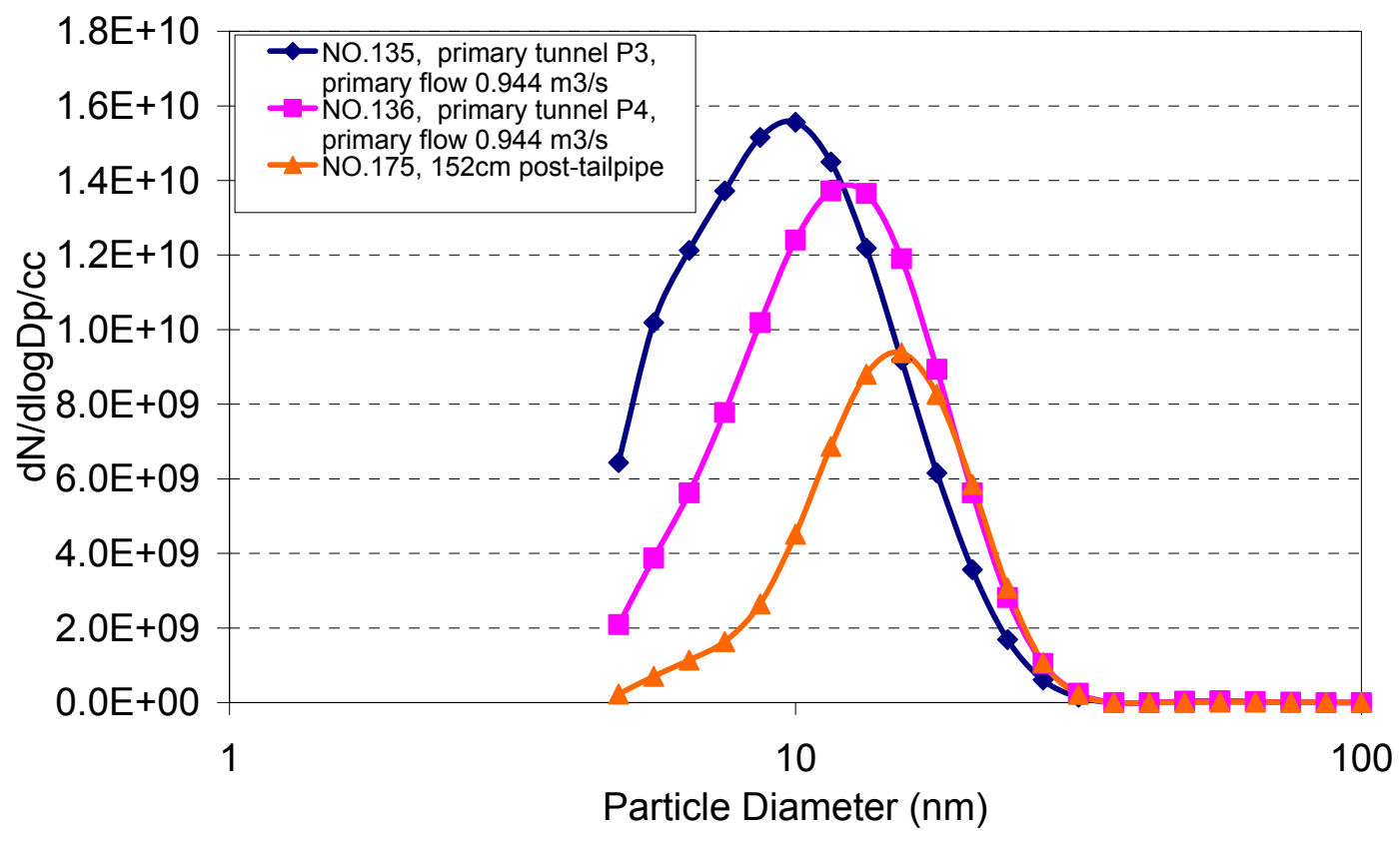

Figure 104 Particle size distributions of tunnel and post-tailpipe test at dilution ratios of 31.5 (102 Nm @ 1800 rpm)

Particle size distributions measured inside the primary tunnel shown in Figure 101 and Figure 102 had higher peaks than those measured at same dilution ratios posttailpipe, with similar shapes. Particle size distributions measured at the secondary 
dilution tunnel outlet were shifted to large diameter end compared to the other two curves in both figures. Particle size distributions measured inside the primary tunnel shown in Figure 103 shifted to the left side and still had a higher peak than the post-tailpipe particle size distribution. Due to the different dilution conditions such as dilution air temperature, dilution speed, and residence time, obvious differences existed for the particle size distributions measured at the same dilution ratios between tunnel test and post-tailpipe test. Similar difference in particle size distributions at the same dilution ratio were also observed when the engine was running at 102Nm @ 1800 rpm. Figure 104 shows the stack equivalent particle size distribution curves of tunnel and post-tailpipe test at dilution ratio 31.5 when the engine was running at $102 \mathrm{Nm}$.

In Figure 104, the particle size distribution shapes' shifting from number 135 to number 136 was the same phenomenon illustrated in the early tunnel test discussion as Figure 73. Similar to test results compared for engine running at $122 \mathrm{Nm}$, the posttailpipe particle size distribution measured at the same dilution ratios 31.5 had a lower and right-shifted peak compared to size distributions measured inside the tunnel. To investigate same dilution ratios, stack equivalent particle mass concentration levels and number concentration levels are shown in Figure 105 and Figure 106.

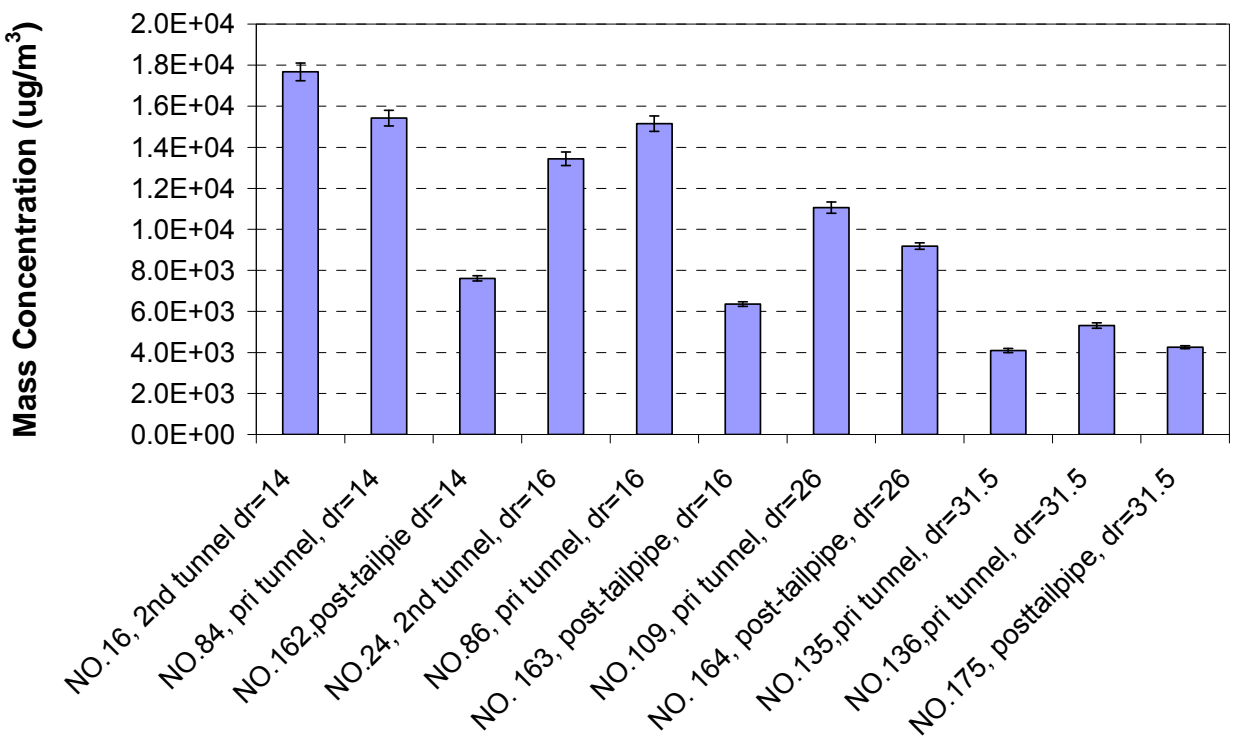

Figure 105 Particle mass concentration levels for tunnel and post-tailpipe test at same dilution ratios 


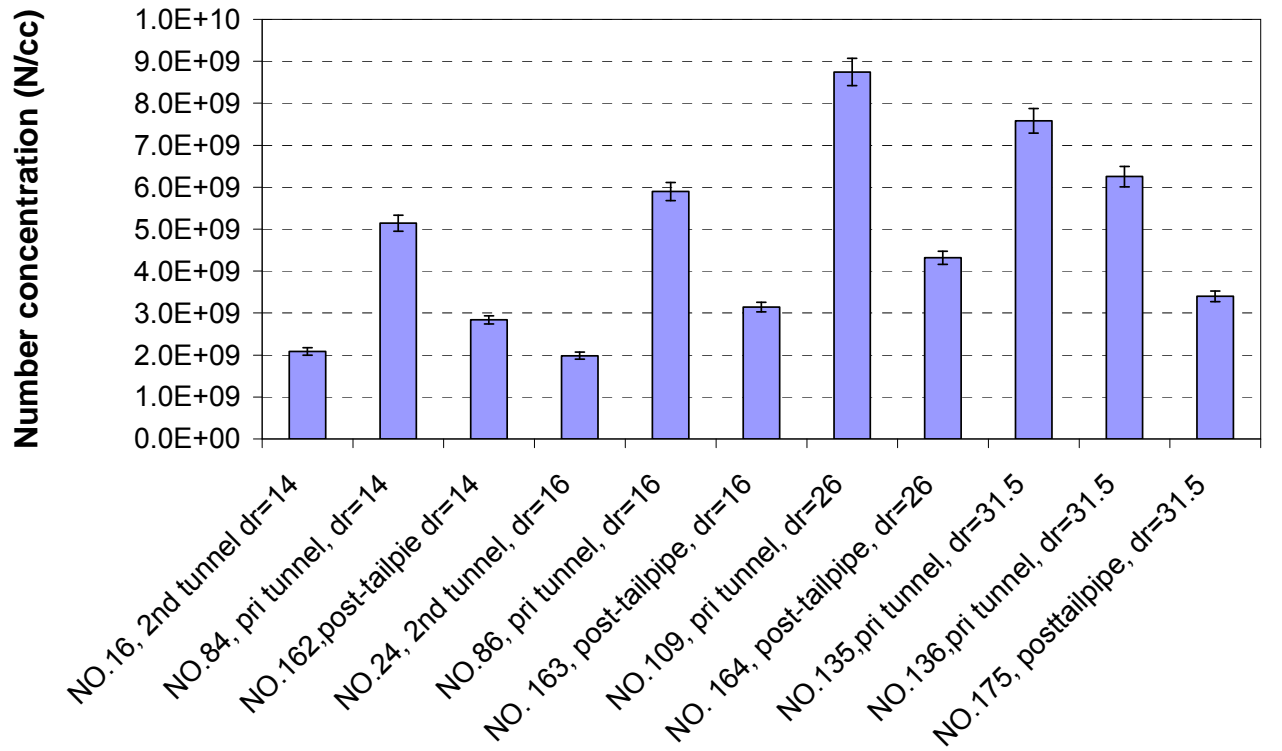

Figure 106 Particle number concentration levels for tunnel and post-tailpipe test at the same dilution ratios

For the investigated dilution ratios, the particle mass concentration levels measured post-tailpipe at the same dilution ratios were generally lower than those measured using the dilution tunnels. The particle number concentration levels measured post-tailpipe at the same dilution ratio were generally higher than those measured at the secondary tunnel outlet and lower than those measured inside the primary tunnel. It is worth pointing out that the conclusions for general change trend for particle number and mass concentration levels were only drawn from examined dilution ratios at examined sample locations. Even at the same dilution ratios condition for the same dilution method, DPM evolution went on. Particle size distributions measured at other dilution ratios for different dilution method might display different behaviors for the trends of distribution shape, number and mass concentration level. Such consideration applies to comparison between dilution tunnels, PPMD, and post-tailpipe tests.

\section{b. Tunnel vs. PPMD Test}

Figure 107 and Figure 108 compare the stack equivalent particle size distribution curves of tunnel and PPMD test when the engine was running at 102Nm @1800 rpm and 122Nm@1800 rpm, respectively. The tunnel test part was focused on the secondary dilution tunnel measurement. Corresponding stack equivalent particle mass concentration 
and number concentration levels are illustrated in Figure 109 and Figure 110 for investigated dilution ratios.

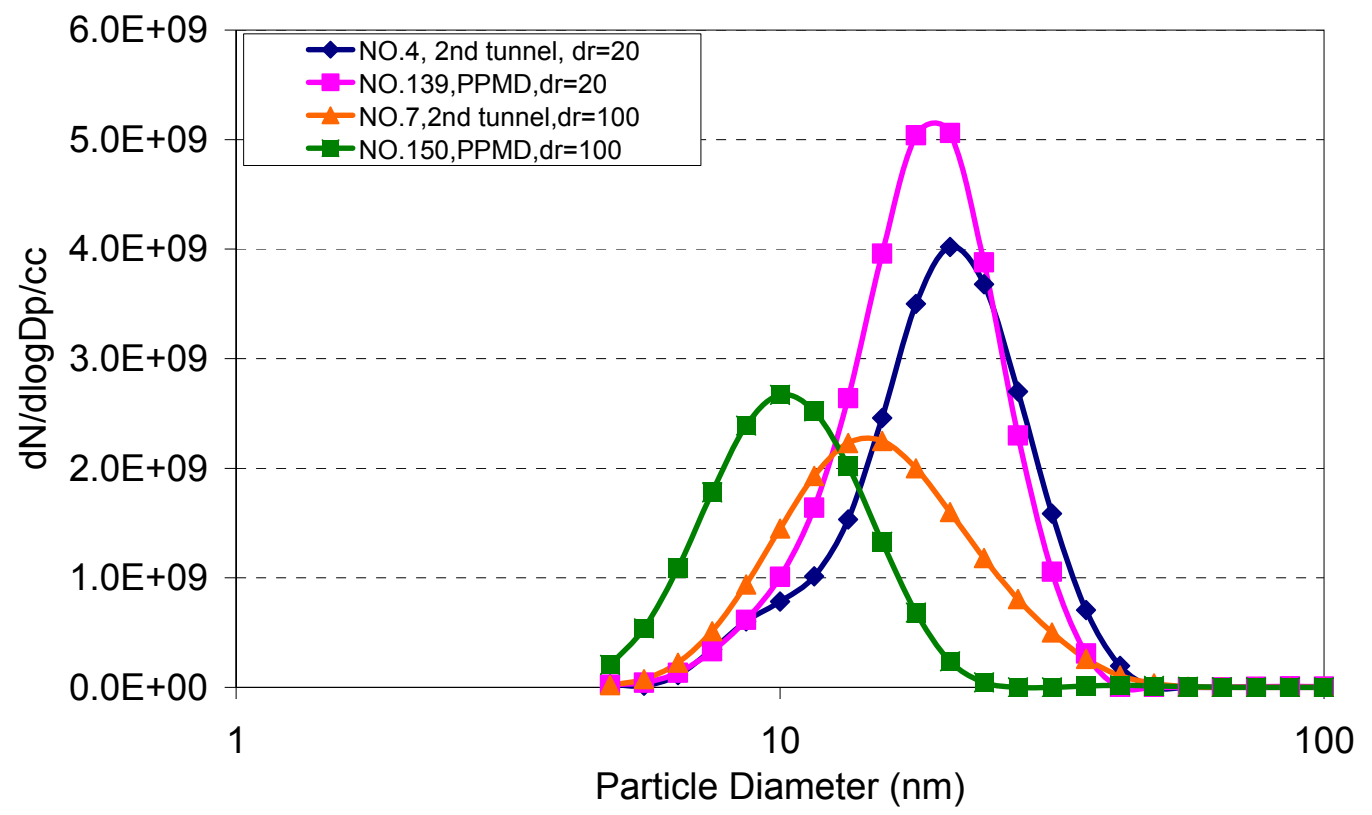

Figure 107 Particle size distributions of tunnel and PPMD test (102 Nm @ 1800 rpm)

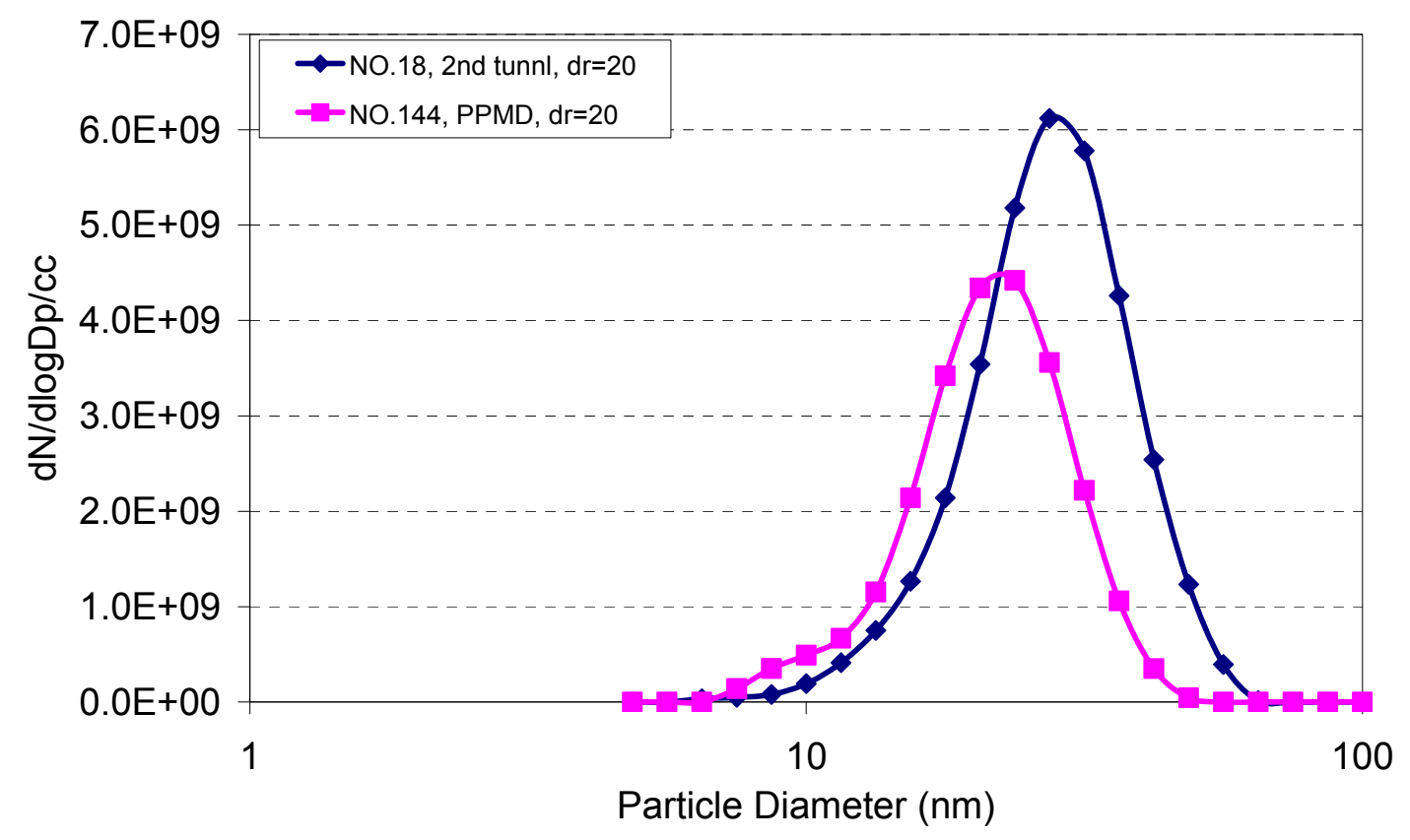

Figure 108 Particle size distributions of tunnel and PPMD test (122 Nm @ 1800 rpm) 


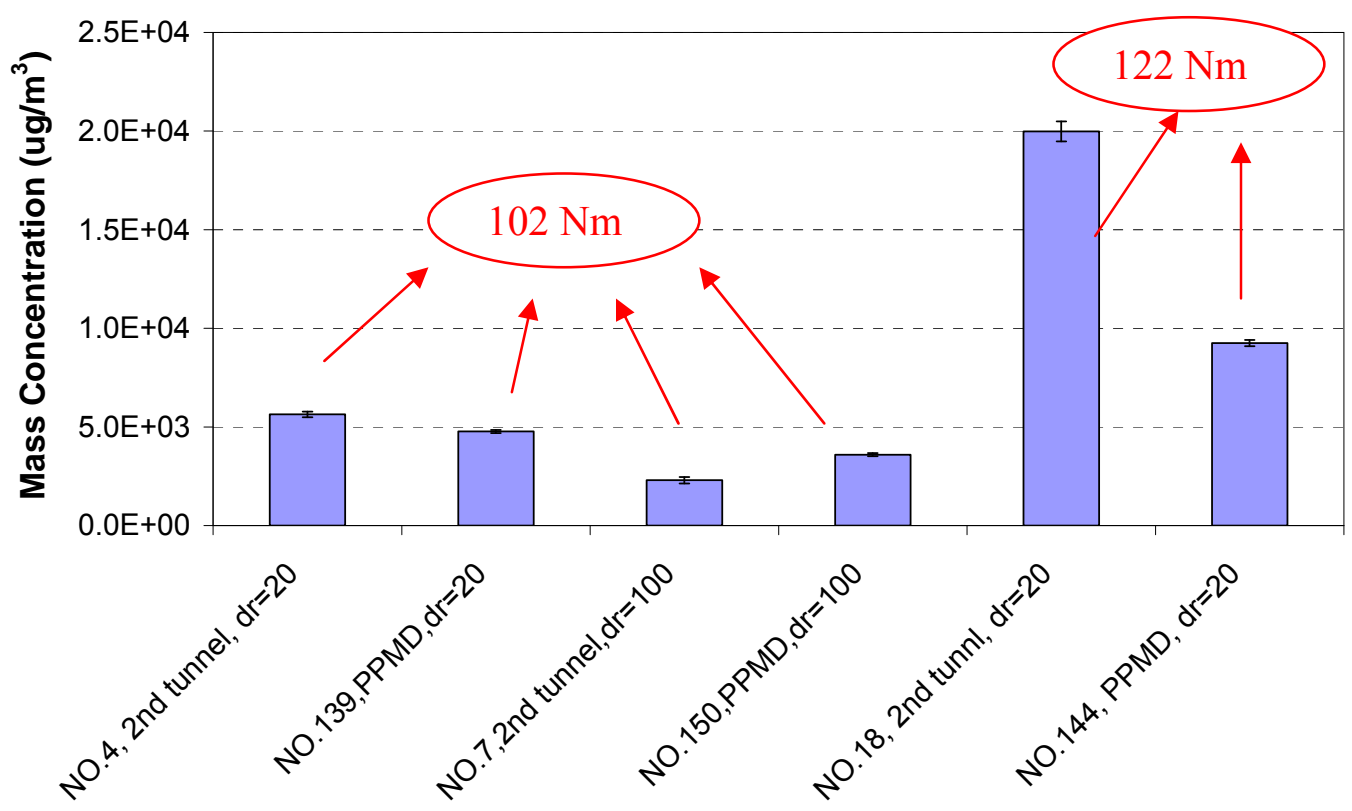

Figure 109 Particle mass concentration levels for tunnel and PPMD test at the same dilution ratios

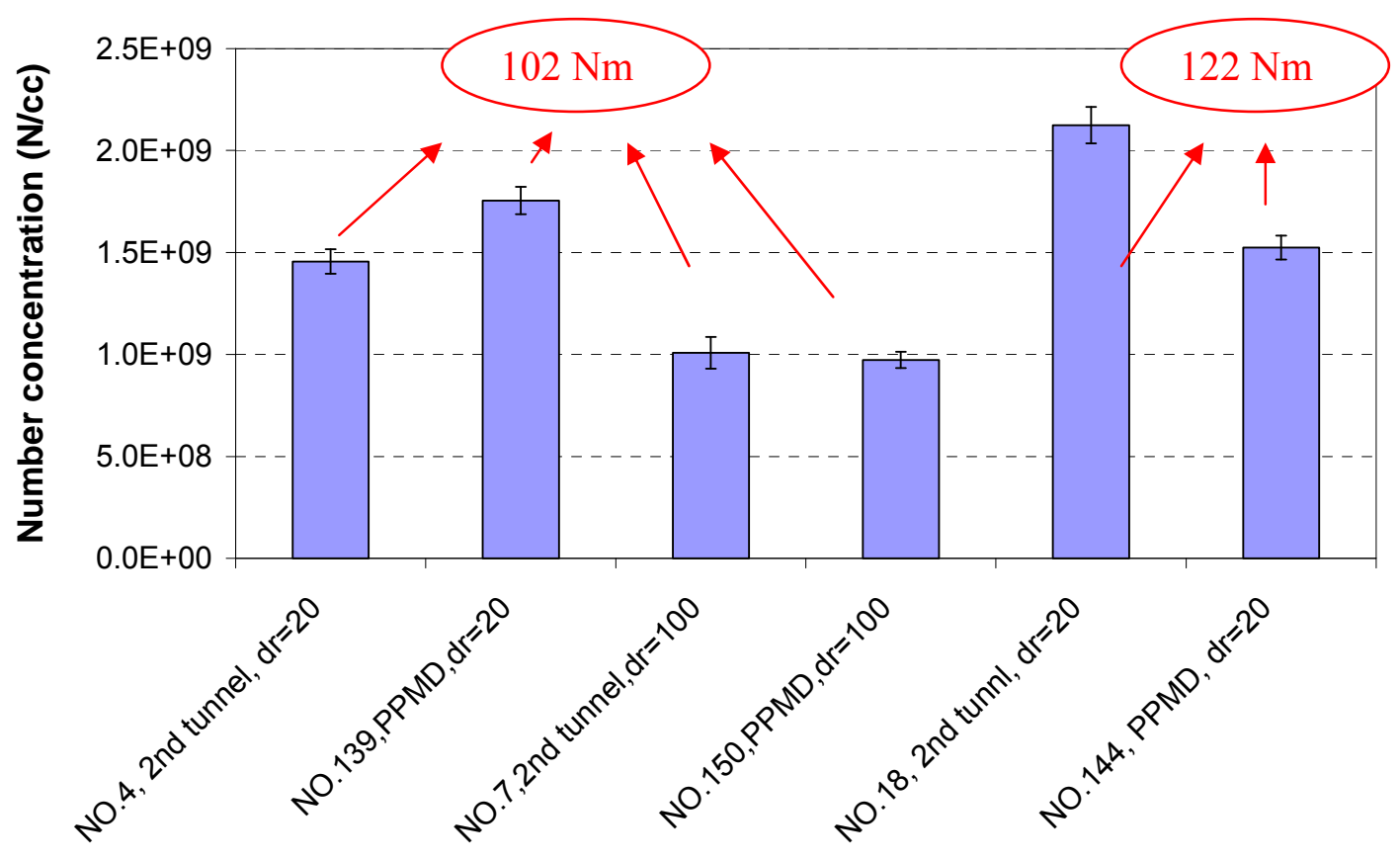

Figure 110 Particle number concentration levels for tunnel and PPMD test at the same dilution ratios

Particle size distributions measured at the outlet of secondary dilution tunnel with dilution ratio 20 show a higher peak than the particle size distributions measured from 
PPMD at the same dilution ratio with engine torque setting $122 \mathrm{Nm}$ and a lower peak with engine torque setting $102 \mathrm{Nm}$. Correspondingly, particle number concentration level at the outlet of secondary dilution tunnel with dilution ratio 20 were higher than that of PPMD measurement at $102 \mathrm{Nm}$ torque setting and lower than that of PPMD measurement at $122 \mathrm{Nm}$ torque setting. However, particle mass concentration levels at the secondary tunnel out for both engine torque settings were higher than those of PPMD measurement.

When the dilution ratio was increased to 100, while the PPMD was operating at dual MPS diluters stage mode, both particle size distributions measured from the secondary tunnel outlet and from the PPMD displayed lower peaks and shifted to lower diameter side compared to particle size distributions measured at dilution ratio 20. Both particle size distribution number concentration levels were almost equal to each other. Compared to particle size distribution measured at dilution ratio 20, more particle mass was lost with dilution ratio 100. And particle loss from PPMD measurement was less than that of secondary tunnel measurement.

\section{c. Post-tailpipe vs. PPMD Test}

Figure 111 and Figure 112 show the stack equivalent particle size distribution curves of post-tailpipe and PPMD test when the engine was running at 102Nm @ 1800 rpm and 122Nm@1800 rpm, respectively. Corresponding stack equivalent particle mass concentration and number concentration levels are illustrated in Figure 113 and Figure 114 for investigated dilution ratios.

Particle size distributions shown in Figure 111 displayed similar shapes for posttailpipe and PPMD tests at dilution ratios 10 and 12, with slight difference in peak height or peak diameter. The corresponding particle number and mass concentration levels shown in Figure 113 and Figure 114 for the PPMD test were lower than the post-tailpipe test when the engine was operated at $102 \mathrm{Nm} @ 1800 \mathrm{rpm}$. However, when the engine was running at $122 \mathrm{Nm}$, the post-tailpipe measured particle size distribution at dilution ratio 8 was left-shifted with a lower shape peak compared to PPMD test. The 
corresponding particle number and mass concentration levels of PPMD test were higher than those of the post-tailpipe test at dilution ratio 8 .

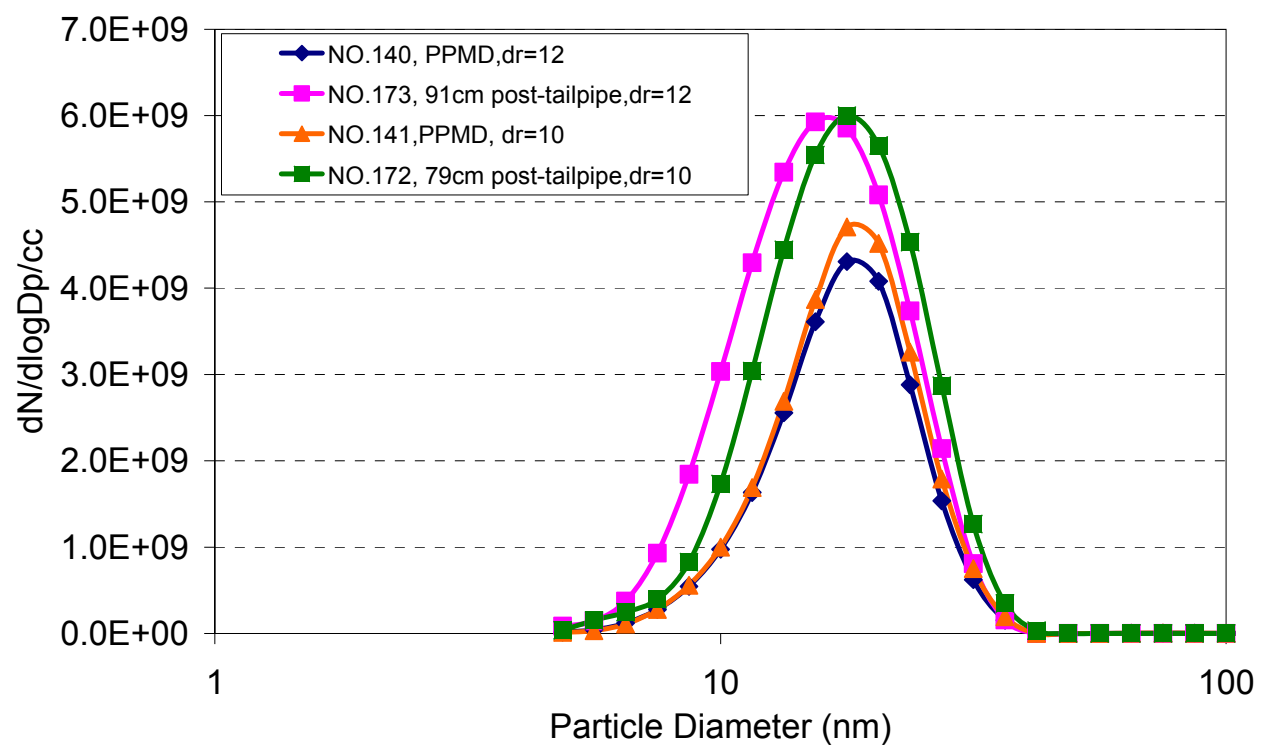

Figure 111 Particle size distributions of post-tailpipe and PPMD test (102 Nm @ $1800 \mathrm{rpm})$

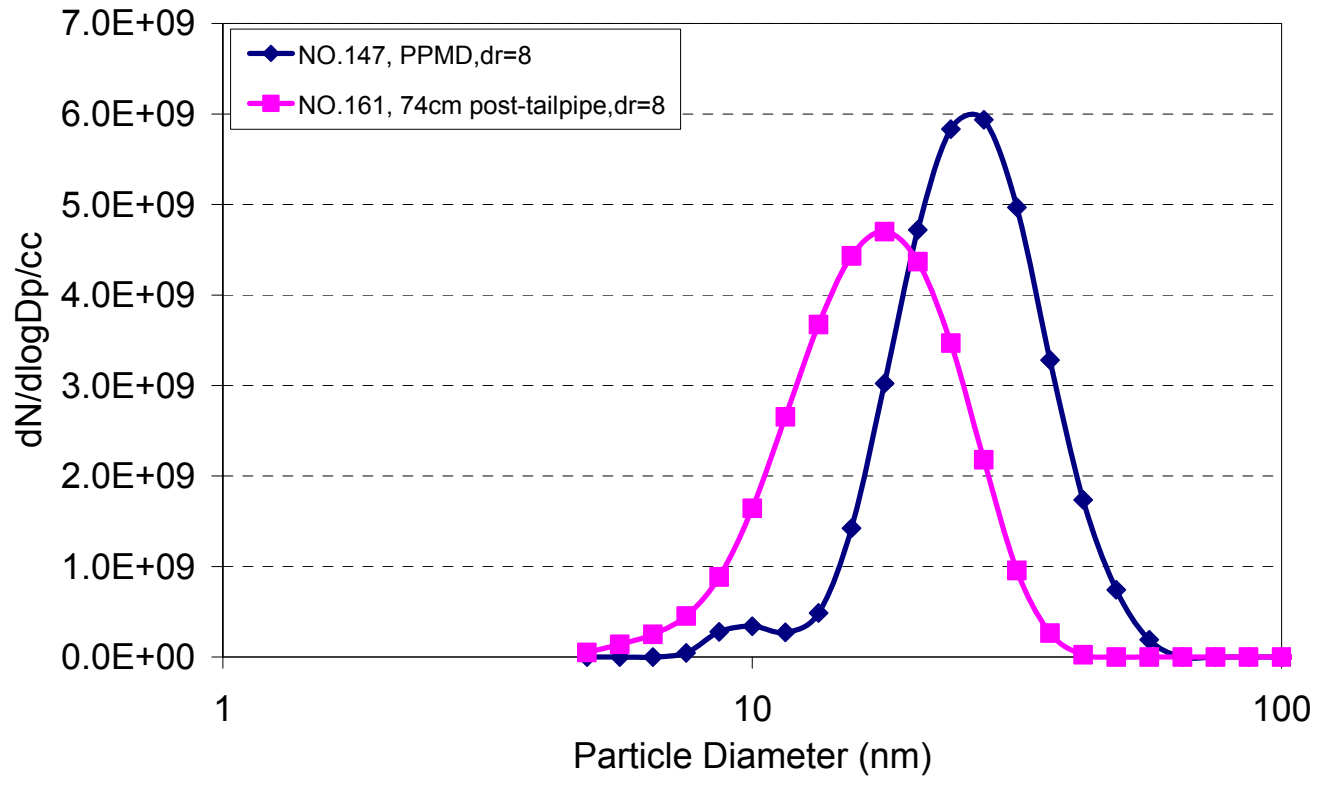

Figure 112 Particle size distributions of post-tailpipe and PPMD test (122 Nm @ 1800 rpm) 


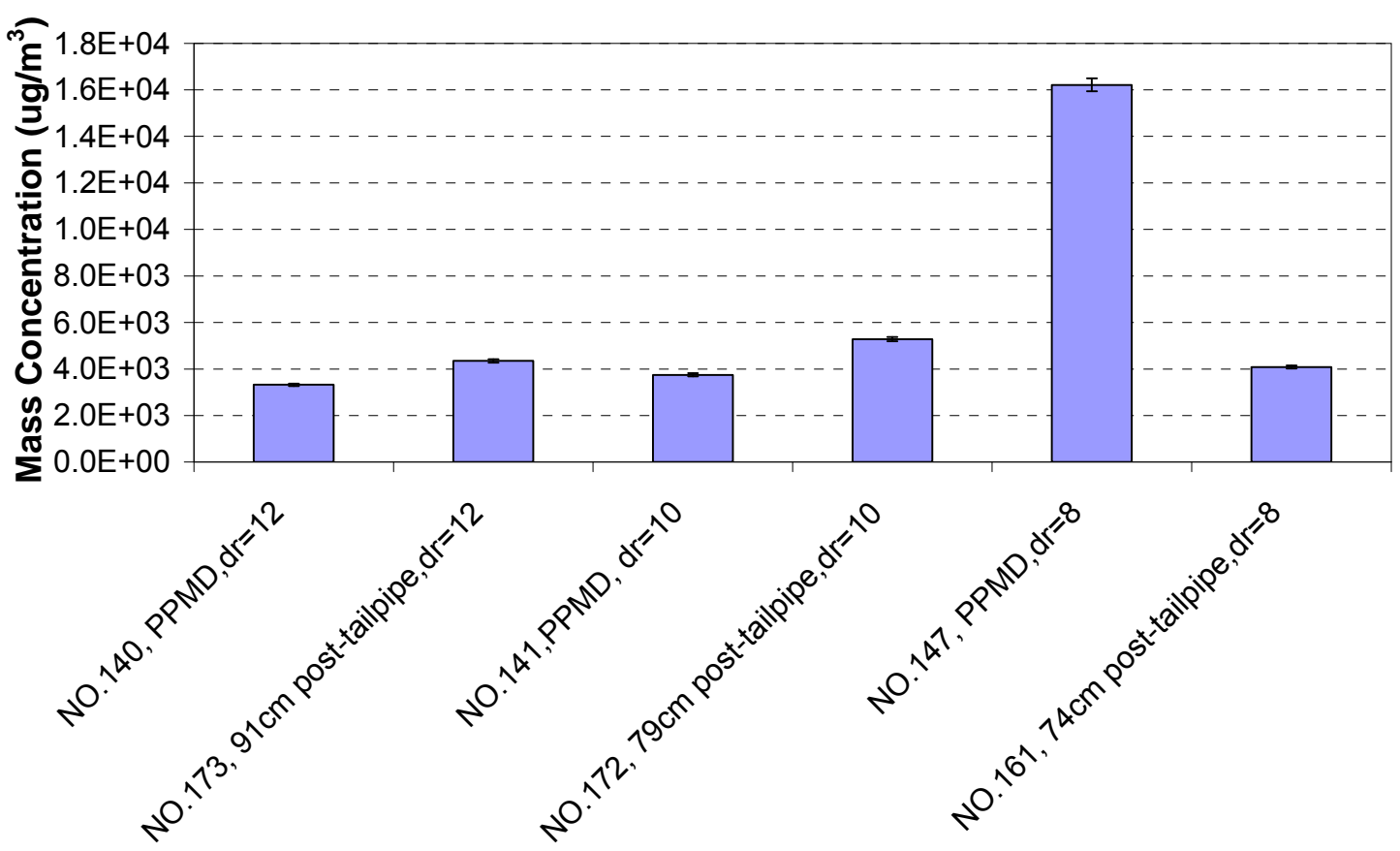

Figure 113 Particle mass concentration levels for post-tailpipe and PPMD test at the same dilution ratios

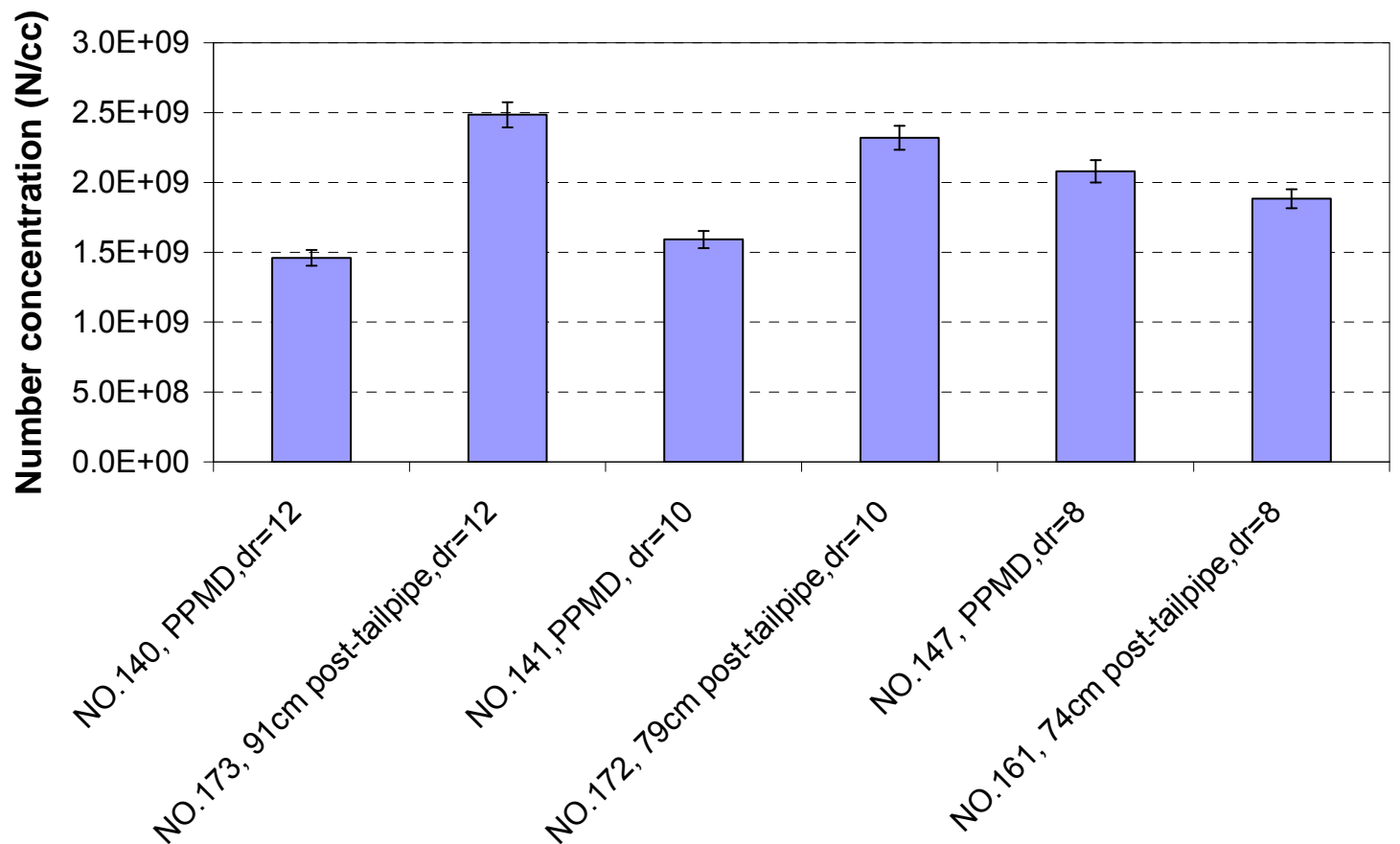

Figure 114 Particle number concentration levels for post-tailpipe and PPMD test at the same dilution ratios 


\subsubsection{Distributions Remap}

The secondary part of comparison of different dilution method effects on the particle size distribution was performed to find same particle size distributions in terms of shape, number and mass concentration levels for different dilution methods. The comparison was conducted for both engine stack corrected and uncorrected particle size distributions. The stack corrected value comparison was based on the total particle emission point of view and the information would be more preferable to emissions standard regulators. However, the knowledge acquired from the uncorrected values would be valuable to target PM emission hot spots for individuals, especially for the exhaust plume studied post-tailpipe. Stack corrected and uncorrected particle size distributions were summarized in Table A2 in the Appendix and were compared to each other to find remapped size distributions. Particle size distributions were first compared to each other in terms of number and mass concentration levels. Only pairs of size distributions that were within $15 \%$ difference in number concentration levels and mass concentration levels were further analyzed for comparison of shapes. Particle size distributions were all normalized to have an area under the curve of 1 so that the Kolmogorov Smirnov two-sample test method and least square root method could be applied.

\section{a. Kolmogorov Smirnov two-sample test method}

In order to apply the Kolmogorov Smirnov two-sample test method, a 300 point sample was randomly generated for each measured particle size distribution using the normalized particle size distribution curve as the distribution PDF. The number of sample points was carefully chosen to provide enough accuracy for asymptotic p-value determination and to avoid introducing too much sample noise. Pairs of stack corrected and uncorrected particle size distributions with number and mass concentration level tolerances were tested. The two-sample Kolmogorov Smirnov test compared the vectors of two 300 point samples $\mathrm{X} 1$ and $\mathrm{X} 2$. The null hypothesis is $\mathrm{X} 1$ and $\mathrm{X} 2$ were randomly generated from the same continuous distribution. The alternative hypothesis is they were from different distributions. A 5\% significant level was applied during the hypothesis test procedures. 
Table 6 and Table 7 list the hypothesis test results for pairs of distributions with null hypothesis not being rejected of stack uncorrected and corrected pairs of particle distributions. The particle size distributions remapping was focus on size distributions of different dilution methods at same engine running conditions. Table 6 shows that for stack uncorrected particle size distributions, it was possible to remap certain post-tailpipe measured size distributions to tunnel and PPMD measurement results under the prescribed criteria. Table 7 shows that for stack corrected particle size distributions, certain secondary tunnel outlet measured size distributions were able to match posttailpipe and PPMD test results. There were particle size distributions that could be remapped to others obtained in the same dilution method and were not summarized in Table 6 and Table 7.

Table 6 Stack uncorrected particle size distributions remap (Kolmogorov Smirnov test)

\begin{tabular}{|c|c|c|c|c|c|}
\hline Distribution A & Distribution B & P-value & Statistics & $\begin{array}{l}\text { Difference of } \\
\text { number } \\
\text { concentration } \\
(\mathrm{A}-\mathrm{B}) / \mathrm{B} \times 100 \%\end{array}$ & $\begin{array}{l}\text { Difference of } \\
\text { mass } \\
\text { concentration } \\
(\mathrm{A}-\mathrm{B}) / \mathrm{B} \times 100 \%\end{array}$ \\
\hline $\begin{array}{l}\text { NO. 92, Primary tunnel } \mathrm{P} 2 \text {, } \\
22.9 \mathrm{~cm} \text { from center, } \mathrm{dr}=18 \text {, } \\
\text { primary flow rate } 800 \mathrm{SCFM} \\
\left(0.378 \mathrm{~m}^{3} / \mathrm{s}\right) \text {, engine running at } \\
122 \mathrm{Nm}\end{array}$ & $\begin{array}{l}\text { NO. } 160,61 \mathrm{~cm} \text { post- } \\
\text { tailpipe, } \mathrm{dr}=6.5 \text {, engine } \\
\text { running at } 122 \mathrm{Nm}\end{array}$ & 0.17 & 0.090 & 1.4 & 13.0 \\
\hline $\begin{array}{l}\text { NO. } 102 \text {, Primary tunnel } \mathrm{P} 1 \text {, } \\
12.2 \mathrm{~cm} \text { from center, } \mathrm{dr}=23 \text {, } \\
\text { primary flow rate } 800 \mathrm{SCFM} \\
\left(0.378 \mathrm{~m}^{3} / \mathrm{s}\right) \text {, engine running at } \\
122 \mathrm{Nm}\end{array}$ & $\begin{array}{l}\text { NO. } 160,61 \mathrm{~cm} \text { post- } \\
\text { tailpipe, } \mathrm{dr}=6.5 \text { engine } \\
\text { running at } 122 \mathrm{Nm}\end{array}$ & 0.09 & 0.100 & 13.4 & 5.0 \\
\hline $\begin{array}{l}\text { NO. } 103 \text {, Primary tunnel } \mathrm{P} 1 \text {, } \\
15 \mathrm{~cm} \text { from center, } \mathrm{dr}=22 \text {, } \\
\text { primary flow rate } 800 \mathrm{SCFM} \\
\left(0.378 \mathrm{~m}^{3} / \mathrm{s}\right) \text {, engine running at } \\
122 \mathrm{Nm}\end{array}$ & $\begin{array}{l}\text { NO. } 160,61 \mathrm{~cm} \text { post- } \\
\text { tailpipe, } \mathrm{dr}=6.5 \text { engine } \\
\text { running at } 122 \mathrm{Nm}\end{array}$ & 0.44 & 0.070 & 12.9 & 5.3 \\
\hline $\begin{array}{l}\text { NO. } 142, \quad \text { PPMD } \\
\text { dilution ratio } d r=7, \\
\text { running at } 102 \mathrm{Nm}\end{array}$ & $\begin{array}{l}\text { NO. } 174,109 \mathrm{~cm} \text { post- } \\
\text { tailpipe, } \mathrm{dr}=15.3 \text { engine } \\
\text { running at } 102 \mathrm{Nm}\end{array}$ & 0.57 & 0.063 & 6.4 & 5.4 \\
\hline
\end{tabular}


Table 7 Stack corrected particle size distributions remap (Kolmogorov Smirnov test)

\begin{tabular}{|c|c|c|c|c|c|}
\hline Distribution A & Distribution B & P-value & Statistics & $\begin{array}{l}\text { Difference of } \\
\text { number } \\
\text { concentration } \\
\text { (A-B)/B } \times 100 \%\end{array}$ & $\begin{array}{l}\text { Difference of } \\
\text { mass } \\
\text { concentration } \\
(\mathrm{A}-\mathrm{B}) / \mathrm{B} \times 100 \%\end{array}$ \\
\hline $\begin{array}{l}\text { NO. } 6 \text {, secondary tunnel outlet } \\
\text { with total } \mathrm{dr}=50 \text {, secondary } \\
\text { flow rate of } 2.0 \mathrm{SCFM}(56.6 \\
\text { lpm), engine running at } 102 \mathrm{Nm}\end{array}$ & $\begin{array}{l}\text { NO. } 169,28 \mathrm{~cm} \text { post } \\
\text { tail-pipe, } \mathrm{dr}=4.2 \text { engine } \\
\text { running at } 102 \mathrm{Nm}\end{array}$ & 0.08 & 0.103 & 0.1 & 13.7 \\
\hline $\begin{array}{l}\text { NO. 16, secondary tunnel } \\
\text { outlet with total } \mathrm{dr}=14.3 \text {, } \\
\text { secondary flow rate of } 2.0 \\
\operatorname{SCFM}(56.6 \mathrm{lpm}) \\
\text { engine running at } 122 \mathrm{Nm}\end{array}$ & $\begin{array}{l}\text { NO. 147, PPMD single } \\
\text { dilution ratio } \mathrm{dr}=10 \text {, } \\
\text { engine running at } \\
122 \mathrm{Nm}\end{array}$ & 0.77 & 0.053 & 0.3 & 8.2 \\
\hline $\begin{array}{l}\text { NO. } 17, \text { secondary tunnel } \\
\text { outlet with total } \mathrm{dr}=16.7 \text {, } \\
\text { secondary flow rate of } 2.0 \\
\text { SCFM }(56.6 \mathrm{lpm}) \text { engine } \\
\text { running at } 122 \mathrm{Nm}\end{array}$ & $\begin{array}{l}\text { NO. 147, PPMD single } \\
\text { dilution ratio dr=10, } \\
\text { engine running at } \\
122 \mathrm{Nm}\end{array}$ & 0.44 & 0.070 & 4.4 & 1.9 \\
\hline $\begin{array}{l}\text { NO. 23, secondary tunnel } \\
\text { outlet with total } \mathrm{dr}=14.1 \text {, } \\
\text { secondary flow rate of } 2.0 \\
\text { SCFM }(63.7 \mathrm{lpm}) \text { engine } \\
\text { running at } 122 \mathrm{Nm}\end{array}$ & $\begin{array}{l}\text { NO. 147, PPMD single } \\
\text { dilution ratio } \mathrm{dr}=10 \text {, } \\
\text { engine running at } \\
122 \mathrm{Nm}\end{array}$ & 0.83 & 0.050 & 1.1 & 1.8 \\
\hline $\begin{array}{l}\text { NO. 21, secondary tunnel } \\
\text { outlet with total } \mathrm{dr}=100 \text {, } \\
\text { secondary flow rate of } 2.0 \\
\text { SCFM }(56.6 \mathrm{lpm}) \text {, engine } \\
\text { running at } 122 \mathrm{Nm}\end{array}$ & $\begin{array}{l}\text { NO. } 159,38 \mathrm{~cm} \text { post } \\
\text { tail-pipe, } \mathrm{dr}=4.2 \text {, engine } \\
\text { running at } 122 \mathrm{Nm}\end{array}$ & 0.17 & 0.090 & 0.4 & 11.6 \\
\hline
\end{tabular}

\section{b. Least square root method}

Table 8 and Table 9 show the remapped particle size distributions for different dilution methods using least square root method. A maximum of 0.2 least square root values was applied. The preset maximum value of the least square root determined how many pairs of size distributions were consider being same. Matched pairs of particle size distributions in Table 8 and Table 9 were found to be subsets of particle size distributions shown in Table 6 and Table 7. Further discussion then was focus on results from 
Kolmogorov Smirnov test method results. To give examples of how the remapped size distribution shapes compared to each other, two pairs of remapped size distributions for stack uncorrected and corrected are shown in Figure 115 and Figure 116.

Table 8 Stack uncorrected particle size distributions (least square root test)

\begin{tabular}{|c|c|c|c|c|}
\hline Distribution A & Distribution B & $\begin{array}{l}\text { Least square } \\
\text { root value }\end{array}$ & $\begin{array}{l}\text { Difference of } \\
\text { number } \\
\text { concentration } \\
(\mathrm{A}-\mathrm{B}) / \mathrm{B} \times 100 \%\end{array}$ & $\begin{array}{l}\text { Difference of mass } \\
\text { concentration } \\
(\mathrm{A}-\mathrm{B}) / \mathrm{B} \times 100 \%\end{array}$ \\
\hline $\begin{array}{l}\text { NO. } 92 \text {, Primary tunnel } \mathrm{P} 2,22.9 \\
\mathrm{~cm} \text { from center, } \mathrm{dr}=18 \text {, primary } \\
\text { flow rate } 800 \mathrm{SCFM}(0.378 \\
\left.\mathrm{m}^{3} / \mathrm{s}\right) \text {, engine running at } 122 \mathrm{Nm}\end{array}$ & $\begin{array}{l}\text { NO. } 160,61 \mathrm{~cm} \text { post-tailpipe, } \\
\mathrm{dr}=6.5 \text {, engine running at } \\
122 \mathrm{Nm}\end{array}$ & 0.11 & 1.4 & 13.0 \\
\hline $\begin{array}{l}\text { NO. } 102 \text {, Primary tunnel } \mathrm{P} 1,12.2 \\
\mathrm{~cm} \text { from center, dr=23, primary } \\
\text { flow rate } 800 \mathrm{SCFM}(0.378 \\
\left.\mathrm{m}^{3} / \mathrm{s}\right) \text {, engine running at } 122 \mathrm{Nm}\end{array}$ & $\begin{array}{l}\text { NO. } 160,61 \mathrm{~cm} \text { post-tailpipe, } \\
\mathrm{dr}=6.5 \text { engine running at } \\
122 \mathrm{Nm}\end{array}$ & 0.18 & 13.4 & 5.0 \\
\hline $\begin{array}{l}\text { NO. } 142 \text {, PPMD single dilution } \\
\text { ratio } \mathrm{dr}=7 \text {, engine running at } \\
102 \mathrm{Nm}\end{array}$ & $\begin{array}{l}\text { NO. } 174,109 \mathrm{~cm} \text { post-tailpipe, } \\
\mathrm{dr}=15.3 \text { engine running at } \\
102 \mathrm{Nm}\end{array}$ & 0.19 & 6.4 & 5.4 \\
\hline
\end{tabular}

Table 9 Stack corrected particle size distributions remap (least square root test)

\begin{tabular}{|c|c|c|c|c|}
\hline Distribution A & Distribution B & $\begin{array}{l}\text { Least square } \\
\text { root value }\end{array}$ & $\begin{array}{l}\text { Difference of } \\
\text { number } \\
\text { concentration } \\
(\mathrm{A}-\mathrm{B}) / \mathrm{B} \times 100 \%\end{array}$ & $\begin{array}{l}\text { Difference of mass } \\
\text { concentration } \\
(\mathrm{A}-\mathrm{B}) / \mathrm{B} \times 100 \%\end{array}$ \\
\hline $\begin{array}{l}\text { NO. } 16 \text {, secondary tunnel outlet } \\
\text { with total } \mathrm{dr}=14.3 \text {, secondary } \\
\text { flow rate of } 2.0 \mathrm{SCFM}(56.6 \\
\text { lpm), engine running at } 122 \mathrm{Nm}\end{array}$ & $\begin{array}{l}\text { NO. } 147 \text {, PPMD single dilution } \\
\text { ratio } d r=10 \text {, engine running at } \\
122 \mathrm{Nm}\end{array}$ & 0.19 & 0.3 & 8.2 \\
\hline $\begin{array}{l}\text { NO. } 17 \text {, secondary tunnel outlet } \\
\text { with total } \mathrm{dr}=16.7 \text {, secondary } \\
\text { flow rate of } 2.0 \mathrm{SCFM}(56.6 \\
\text { lpm), engine running at } 122 \mathrm{Nm}\end{array}$ & $\begin{array}{l}\text { NO. } 147 \text {, PPMD single dilution } \\
\text { ratio } d r=10 \text {, engine running at } \\
122 \mathrm{Nm}\end{array}$ & 0.17 & 4.4 & 1.9 \\
\hline $\begin{array}{l}\text { NO. } 23 \text {, secondary tunnel outlet } \\
\text { with total } \mathrm{dr}=14.1 \text {, secondary } \\
\text { flow rate of } 2.0 \mathrm{SCFM}(63.7 \\
\mathrm{lpm}) \text {, engine running at } 122 \mathrm{Nm}\end{array}$ & $\begin{array}{l}\text { NO. } 147 \text {, PPMD single dilution } \\
\text { ratio } d r=10 \text {, engine running at } \\
122 \mathrm{Nm}\end{array}$ & 0.1 & 1.1 & 1.8 \\
\hline $\begin{array}{l}\text { NO. } 21 \text {, secondary tunnel outlet } \\
\text { with total } \mathrm{dr}=100 \text {, secondary } \\
\text { flow rate of } 2.0 \mathrm{SCFM}(56.6 \\
\text { lpm), engine running at } 122 \mathrm{Nm}\end{array}$ & $\begin{array}{l}\text { NO. } 159,38 \mathrm{~cm} \text { post-tailpipe, } \\
\mathrm{dr}=4.2 \text {, engine running at } \\
122 \mathrm{Nm}\end{array}$ & 0.17 & 0.4 & 11.6 \\
\hline
\end{tabular}




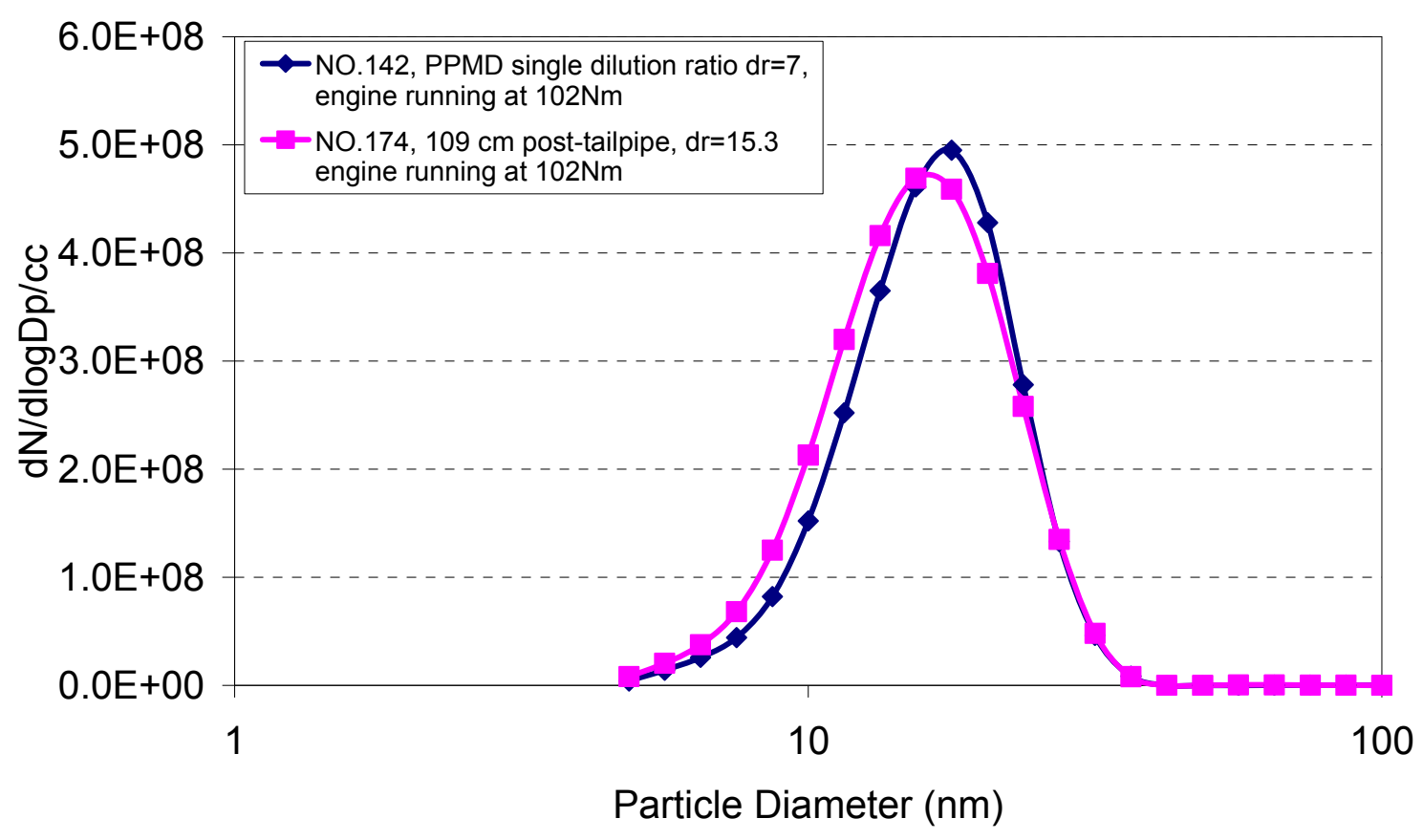

Figure 115 Example of remapped stack uncorrected particle size distributions

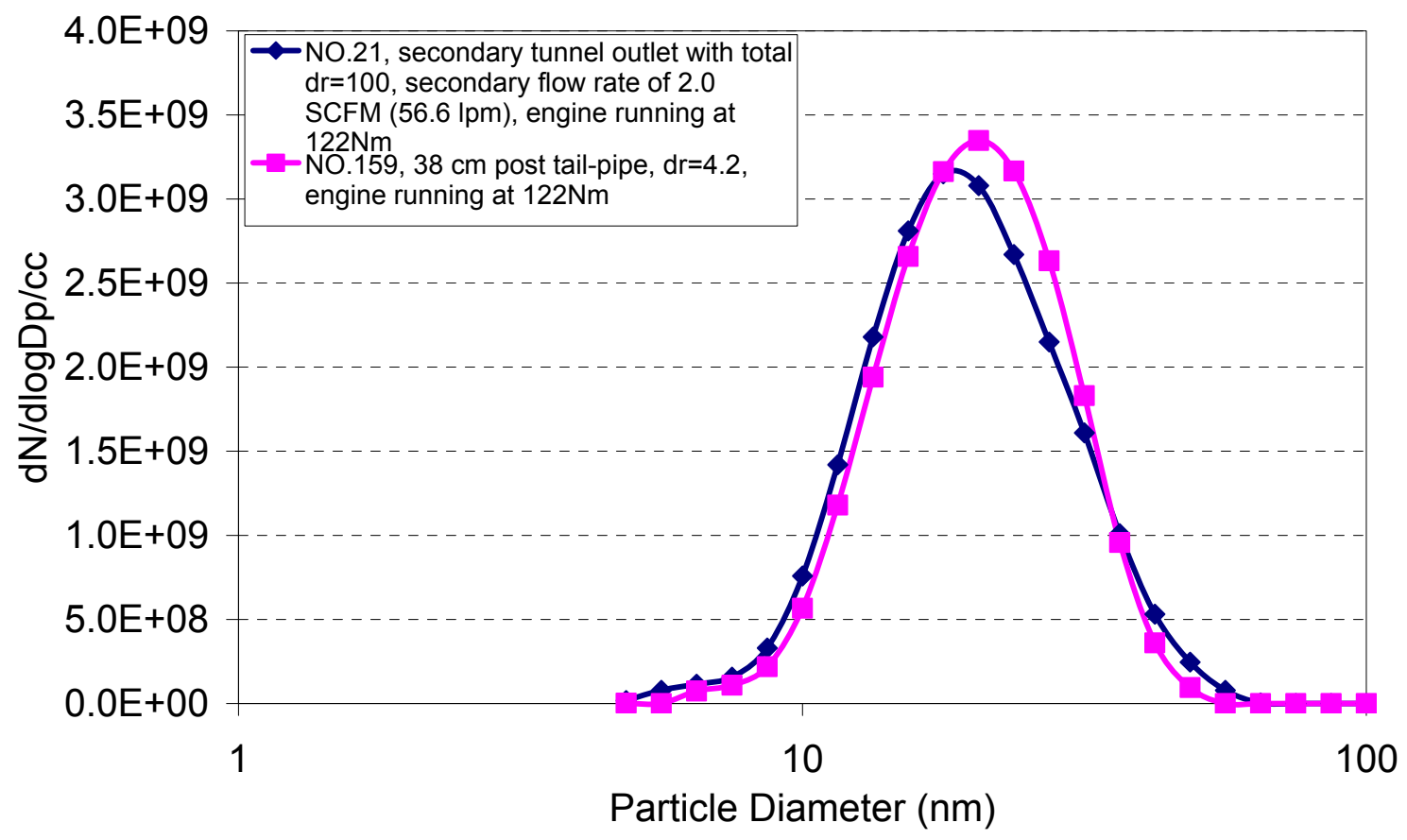

Figure 116 Example of remapped stack corrected particle size distributions

The remapped particle size distributions confirmed that the tunnel dilution and instrument dilution were able to regenerate size distributions of the post-tailpipe dilution at certain locations. Since slight change of the dilution condition would have profound 
effects on particle size distributions, the purpose of finding remapped particle size distributions between different dilution methods, especially between tunnel dilution and post-tailpipe dilution, was not to find the exact locations where the particle size distributions could be mapped. However, remapped particle size distributions for different dilution methods provided information on how tunnel and instrument dilutions mimic fresh emitted PM emissions in term of size distributions. The central hypothesis of this research is that the PM size distributions measured inside the full flow dilution tunnel and at the end of the secondary dilution tunnel can be mapped to the PM size distributions of locations after engine tailpipe in the atmosphere. The analyzed results shown in this section supported the conclusions that this hypothesis could not be rejected. 


\section{CONCLUSIONS AND RECOMMENDATIONS}

This dissertation provides a brief overview of the design and construction techniques used to construct a new transportable laboratory that measures tailpipe gaseous and particulate matter emissions. For the new transportable laboratory to conform to the CFR Part 1065 standard or performance, it is important to have engine fuels, lubricants, and engine work input and output that meet 1065 standards, and so in some circumstances the measurement techniques may be compliant, but the engine may not be configured or exercised in a compoliant fashion. The following conclusions and recommendations can be drawing from the results of this research.

\subsection{Conclusions}

1. The main objective of this research was to advance the understanding of diesel PM formation and measurement in advanced dilution systems and in the real world. This objective was achieved by investigating how the DPM evolves in a full flow CVS tunnel, in the atmosphere post-tailpipe, and in a partial dilution flow device, as well as how the collected DPM size distributions represent the real-world emissions from a diesel engine.

a. This study investigated Diesel particle behaviors for tunnel dilution, PPMD dilution and post-tailpipe dilution. The analysis of particle evolution during dilution was focused on the tunnel dilution test. For the tunnel dilution test, Diesel particle behaviors were examined in the primary and secondary tunnels with varied sampling conditions. For Diesel particles in the nano-size range, a small change in sample conditioning, at times, resulted in a large change of total particle count. This was exemplified by a change in the secondary dilution ratio from 1 to 1.13 , which resulted in a large change in total particle count. When rapid dilution occurred within the flow mixing development sections of the primary tunnel, the mixing of particles may have triggered more nucleation of smaller diameter particles. Higher primary dilution ratios resulted in higher total particle number count and higher mass concentration 
levels. This was probably due to particle nucleation being the dominant transformation process. For secondary dilution ratios between the lowest dilution and a dilution ratio of 3 , there were no significant effects on particle size distribution shapes and mass concentration levels. With high secondary dilution ratios from 3 to 9 , the total particle count and mass concentration levels decreased consistently. Increased residence time of particles in the primary tunnel resulted in less total particle count, but higher mass concentration levels, due to particle size distributions shifting towards larger mean diameters. Residence time of particles in the secondary tunnels had similar effects on the particle characteristics, until a threshold value of residence time was reached. It was anticipated that differences in measured PM mass and test-to-test variation with different dilution ratio settings would be observed.

b. The PPMD and post-tailpipe tests showed that the particle nucleation process occurred more at higher dilution ratios. Post-tailpipe test results showed that the stack equivalent total particle number concentration levels increased with increased distance, up to a $152 \mathrm{~cm}$ post-tailpipe centerline. These results indicate that the nucleation was the dominant process when the exhaust plume was diluted along the post-tailpipe centerline. The measurements taken from the PPMD dilution indicate that change of particle size distribution curves, number, and mass concentration levels were not as strongly correlated to the dilution ratios as two other tests indicated. The PPMD test results showed an irregular pattern of particle number and mass concentration levels with respect to dilution ratios, due to its complex dilution mechanism.

c. Particle size distributions of different dilution methods at the same dilution ratios were compared to each other. The results further revealed that the effects of dilution condition on the particle size distributions were results of combined factors instead of the dilution ratio alone. Further analysis of remapping the tunnel and PPMD measured particle size distributions to the post-tailpipe measurement results show that the CFR dilution process mimics 
freshly emitted engine emissions. The analyzed results showed that stack corrected and uncorrected particle size distributions obtained from one dilution method could be remapped to particle size distributions obtained from another dilution method. The particle size distributions measured inside tunnels could mimic the freshly emitted exhaust immediately post-tailpipe. For example, when the engine was running at $122 \mathrm{Nm}$ and $1800 \mathrm{rpm}$, the stack corrected particle size distribution measurement at $38 \mathrm{~cm}$ of posttailpipe centerline was found to be identical to the stack corrected particle size distribution measurement at the secondary dilution tunnel outlet with the primary dilution ratio of 12.5 and the secondary dilution ratio of 8 .

2. Results were obtained by studying Diesel particle behaviors during tunnel dilution, PPMD dilution and post-tailpipe dilution. The central hypothesis, that the PM size distributions measured inside the full flow dilution tunnel and at the end of the secondary dilution tunnel can be mapped to the PM size distributions at specific locations after the engine tailpipe in the atmosphere, proved to be true. For both stack corrected and uncorrected particle size distributions, a portion of the PPMD and tunnel measurements could be remapped to the post-tailpipe measurements.

3. The second objective of this research, to conduct a parametric study to quantify the design and operating envelope of a PM measurement system in compliance with 2007 standards, and to perform a comparison of study predictions with actual tunnel performance, also was achieved successfully.

a. A detailed parametric study using a Simulink ${ }^{\circledR}$ model to simulate the 2007 PM sampling system was performed to assist the design and manufacture of the transportable laboratory. The Simulink ${ }^{\circledR}$ model was validated by comparing the modeling results to the actual system measurement. The analysis of the simulation results of this model revealed that the simulated venturi and filter face temperatures were comparable with real-world measured ones for a preexisting PM measurement system. 
b. CFD techniques were widely applied in this research. In the design stage of the transportable laboratory, a CFD model was used to find appropriate combinations of the mixing orifice plate size, the exhaust tailpipe location, and the corresponding primary flow rates. For the tunnel test, a CFD model was employed to find optimal locations for the primary tunnel sampling porttailpipe. For the post-tailpipe test, a CFD model was developed to simulate the exhaust plume dispersion and to estimate the ambient wind speed effects on the exhaust dilution. All CFD models were verified with measured dilution ratios and proved able to provide auxiliary information on the exhaust dilution process.

c. There were many compliant designs available within restrictions posed by the EPA's 2007 regulations on PM sampling systems. Settings of test conditions, such as flow rate of the CVS system and wall temperature of the secondary tunnel, are critical to the test system's compliance with the 2007 standards. A test system with the same geometry may or may not meet the 2007 regulations when test conditions change. The same initial test conditions must be set for each FTP test to produce consistent simulation results. The secondary tunnel was responsible for most PM diffusion losses throughout the system. PM diffusion losses mainly occur for particles with diameters less than $10.0 \mathrm{~nm}$. Under most circumstances, particles of such size account for a negligible weight fraction of total PM mass. While the length of transfer pipe had little effect on the filter face temperature, the transfer pipe should be as short as possible in order to reduce heat and PM diffusion loss. Although insulating the transfer pipe and the primary tunnel had little effect on the filter face temperature and PM diffusion losses, insulation can reduce the system's heat loss and is highly recommended. If the stabilized filter face temperatures for the engine running at full power and running at idle are within the specifications of the 2007 standard, it is likely the filter face temperature will be within the restrictions of the 2007 regulations for a transient cycle test. 
4. It is necessary to point out that some conclusions presented from this study may be specific to the engine and emissions aftertreatment device utilized, and, therefore, may not be universally applicable to particle activities for other engine/aftertreatment device combinations.

\subsection{Recommendations}

1. It is recommended that modeling processes are used for the CFR Part 1065 PM measurement system design and/or exhaust dilution process study. Modeling in a Simulink ${ }^{\circledR}$ environment is an effective way to provide assistance to the design of the sampling system. CFD modeling could provide better understanding of exhaust plume dispersion processes.

2. The secondary dilution ratio should be less than 3 to minimize the variation of DPM SOF deposition. It is consistent with the CFR Part 1065's requirements on the secondary dilution ratio (CFR Part 1065 does not regulate explicitly the secondary dilution ratio; it regulates the minimum primary dilution ratio and the range of the minimum total dilution ratio).

3. The post-tailpipe test was conducted in winter with relatively low ambient temperatures. A more through understanding of post-tailpipe DPM formation could be afforded through focus on different meteorological conditions.

4. Future work involving DPM evolution from a different engine and aftertreatment combination, preferably a more recent model year HDDE, is recommended. The posttailpipe study conducted in this reseach was focused on the locations at the posttailpipe centerline; other locations can be investigated for a more thourough understanding of the exhaust plume post-tailpipe dilution and PM formation.

5. Instead of using a DMS500 alone, it is recommended that future investigation of PM evolution also employ a particle number counter further downstream of post-tailpipe locations than the distances conducted in this study. 


\section{REFERENCES}

1. Heyer1 J., Gebhart1 J., and Stahlhofen1 W., "Diameters of Airborne Particles," Water, Air, \& Soil Pol, 1974, Vol. 3-4, pp: 567-572

2. Geier M., Rothen B., Kapp N., Schürch S., Kreyling W., Schulz H., Semmler M., Hof V., Heyder J., and Gehr P., "Ultrafine Particles Cross Cellular Membranes by Nonphagocytic Mechanisms in Lungs and in Cultured Cells," Environ Health Perspect, Nov, 2005, Vol. 113, No. 11, pp: 1555-1560

3. Groneberg A., Morfeld P., Kraus T., Köhler D., Krug N., Magnussen H., Nowak D., Rabe K.F., Schultze-Werninghaus G., Schulz H., Teschler H., Vogelmeier C., Wagner U., Welte T., Voshaar T., and Witt C., "Health Effects of Particulate Matter Exposure: Current Scientific Knowledge,” Pneumologie, Jul, 2009, Vol. 63, No. 7, pp: 363-368

4. Dvonch J.T., Kannan S., Schulz A.J., Keeler G.J., Mentz G., House J., Benjamin A., Max P., Bard R.L., and Brook R.D., "Acute Effects of Ambient Particulate Matter on Blood Pressure: Differential Effects Across Urban Communities," Hypertension, May, 2009, Vol. 53, No. 5, pp: 853-859

5. Maier K.L., Alessandrini F., Beck-Speier I., Hofer T.P., Diabaté S., Bitterle E., Stöger T., Jakob T., Behrendt H., Horsch M., Beckers J., Ziesenis A., Hültner L., Frankenberger M., Krauss-Etschmann S., and Schulz H., "Health Effects of Ambient Particulate Matter - Biological Mechanisms and Inflammatory Responses to in Vitro and in Vivo Particle Exposures," Inhal Toxicol, Feb, 2008, Vol. 20, No. 3, pp: 319-337

6. Lippmann M., "Health Effects of Airborne Particulate Matter," N Engl J Med, 2007, Vol. 357, pp: 2395-2397

7. Samoli E., Peng R., Ramsay T., Pipikou M., Touloumi G., Dominici F., Burnett R., Cohen A., Krewski D., Samet J., and Katsouyanni K., “Acute Effects of Ambient Particulate Matter on Mortality in Europe and North America: Results from the APHENA Study," Environ Health Perspect, Nov, 2008, Vol. 116, No. 11, pp: $1480-1486$ 
8. Kittelson D., Johnson J., Watts W., Wei Q., Drayton M., Paulsen D., and Bukowiecki N., "Diesel Aerosol Sampling in the Atmosphere," SAE Technical Paper No. 2000-01-2212, Warrendale, PA, 2000

9. "Particulate Matter Heath Risk Assessment for Selected Urban Areas," EPA 452/R-05-007A, Dec, 2005

10. Roholm K., "The Fog Disaster in the Meuse Valley, 1930: A Fluorine Intoxication,” J Indus Hyg Toxicol, 1937, Vol. 19, pp: 126-137

11. Camps F.E., "Gradwohl's Legal Medicine, 3rd Edition,” John Wright \& Sons Ltd, Bristol, United Kingdom, 1976, p: 236

12. Hunt A., Abraham J.L., Judson B., and Berry C.L., "Toxicologic and Epidemiologic Clues from the Characterization of the 1952 London Smog Fine Particulate Matter in Archival Autopsy Lung Tissues - Research,” Environ Health Perspect, Jul, 2003, Vol. 111, No. 9, pp: 1209-1214

13. "Carcinogenic Effects of Exposure to Diesel Exhaust," NIOSH Current Intelligence Bulletin 50. DHHS (NIOSH) Publication No. 88-116, Centers for Disease Control, Atlanta, GA

14. "Proposed Identification of Diesel Exhaust as A Toxic Air Contaminant, as Approved by the Scientific Review Panel on April 22, 1998 and Part B: Health Risk Assessment for Diesel Exhaust," CARB/OEHHA, May, 1998

15. "Proposed Identification of Diesel Exhaust as A Toxic Air Contaminant: Initial Statement of Reasons for Rulemaking," CARB/OEHHA, Jun, 1998

16. "Diesel Emissions and Lung Cancer: Epidemiology and Quantitative Risk Assessment - A Special Report of the Institute's Diesel Epidemiology Expert Panel," Health Effects Institute, Cambridge, MA, Jun, 1999

17. "Health Assessment Document for Diesel Engine Exhaust," EPA, EPA/600/890/057F, May, 2002

18. Woodruff T., Grillo J., and Schoendorf K., "The Relationship between Selected Causes of Postneonatal Infant Mortality and Particulate Air Pollution in the United States," Environ Health Perspect, Jun, 1997, Vol. 105, pp: 608-612

19. Janssen N.A.H., Lanki T., Hoek G., Vallius M., Hartog J.J., Grieken R., Pekkanen J., and Brunekreef B., "Associations between Ambient, Personal , and Indoor 
Exposure to Fine Particulate Matter Constituents in Dutch and Finnish Panel of Cardiovascular Patients,” Occup Environ Med, Dec, 2005, Vol. 62, pp: 868-877

20. Peters A., and Pope A.C., "Cardiopulmonary Mortality and Air Pollution," Lancet, Oct, 2002, Vol. 360, No. 9431, pp: 1184-1185

21. Hong Y., Lee J., Kim D.H., Ha E., Schwartz J., and Christiani D.C., "Effects of Air Pollutants on Acute Stroke Mortality," Environ Health Perspect, Feb, 2002, Vol. 110, No. 2, pp: 187-191

22. Kilburn K.H., "Effects of Diesel Exhaust on Neurobehavioral and Pulmonary Functions," Archives of Environmental Health, Jan-Feb, 2000, Vol. 55, No. 1, pp: $11-17$

23. Yin X.J., Shafer R., Ma Jane Y. C., Antonini J.M., Weissman David D., Siegel Paul D., Barger Mark W., Roberts Jenny R., and Ma K.H., “Alteration of Pulmonary Immunity to Listeriamonocytogenes by Diesel Exhaust Particles (Deps). I. Effects of Deps on Early Pulmonary Responses,” Environ Health Perspect, Nov, 2002, Vol. 110, No. 11, pp: 1105-1111

24. Rudell B., Ledin M.C., Hammarstrom U., Stjernberg N., Lundback B., and Sandstrom T., "Effects on Symptoms and Lung Function in Humans Experimentally Exposed to Diesel Exhaust,” Occup Environ Med, Oct, 1996, Vol. 53, No. 10, pp: 658-662

25. Lloyd A.C., and Cackette T.A., "Diesel Engines: Environmental Impact and Control,” J Air Waste Manag Assoc, Jun, 2001, Vol. 51, pp: 809-847

26. "Code of Federal Regulations," Titile 50, National Primary and Secondary Ambient Air Quality Standards, 2007

27. http://ec.europa.eu (accessed on 06/10/200)

28. http://www.env.go.jp

29. Avol E.L., Gauderman W.J., Tan S.M., London S.J., and Peters J.M., "Respiratory Effects of Relocating to Areas of Differing Air Pollution Levels," Am J of Respir Crit Care Med, 2001, Vol. 164, pp: 2067-2072

30. Ritz B., Yu F., Fruin S., Chapa G., Shaw G., and Harris J., “Ambient Air Pollution and Risk of Birth Defects in Southern California," Am J Epidemiol, Jan, 2002, Vol. 155, No. 1, pp: $17-25$ 
31. Johnson P., and Graham J., "Fine Particulate Matter National Ambient Air Quality Standards: Public Health Impact on Populations in the Northeastern United States," Environ Health Perspect, May, 2005, Vol. 113, No. 9, pp: 1140-1147

32. Mysliwiec M.J., and Kleeman M.J., "Source Appointment of Secondary Airborne Particulate Matter in A Polluted Atmosphere," Environ Sci Technol, 2002, Vol. 36, No. 24, pp: 3806-3814

33. Challen B., and Baranescu R., "Diesel Engine Reference Book - Second Edition," Butterworth-Heinemann, Woburn, MA, 1999

34. “Code of Federal Regulations," Title 40, Part 1065

35. Khalek I.A., "2007 Diesel Particulate Measurement Research Final Report, CRC Project-E-66-Phase 1,” May, 2005

36. Khalek I.A., "2007 Diesel Particulate Measurement Research Final Report, CRC Project-E-66-Phase 2," Mar, 2006

37. Khalek I.A., "2007 Diesel Particulate Measurement Research Final Report, CRC Project-E-66-Phase 3," May, 2007

38. "Code of Federal Regulations," Title 40, Part 86

39. http://www.dieselnet.com

40. "Regulatory Impact Analysis: Heavy-Duty Engine and Vehicle Standards and Highway Diesel Fuel Sulfur Control Requirements," EPA, Dec, 2002

41. Kittelson D.B., Watts W.F., Johnson J.P., and Zarling D., "Gasoline Vehicle Exhaust Particulate Sampling Study," Proceedings of U.S. Department of Energy 9th Diesel Engine Emissions Reduction Conference, Newport, RI, Aug 24-28, 2003

42. Bernemyr H., and Ångström H.E., "Number Measurements of Diesel Exhaust Particles - Influence of Dilution and Fuel Sulfur Content," SAE Technical Paper No. 2007-01-0064, Warrendale, PA, 2007

43. Hali D., Thorme C., and Goodier S., "An Investigation into the Effect of A Diesel/Water Emulsion on the Size and Number Distribution of the Particulate Emissions from A Heavy-Duty Diesel Engine," SAE Technical Paper No. 2003-013168, Warrendale, PA, 2003

44. Venkata R.L., Hasan M., and Johnson J., "The Effect of A Diesel Oxidation Catalyst and A Catalyzed Particulate Filter on Particle Size Distribution from A 
Heavy Duty Diesel Engine," SAE Technical Paper No. 2006-01-0877, Warrendale, PA, 2006

45. Kittelson D.B., Arnold M., and Watts W.F., "Review of Diesel Particulate Matter Sampling Methods: Final Report," University of Minnesota, Minneaplolist, MN, Jan, 1999

46. Abbass M.K., Andrews G.E., Ishaq R.B., Williams P.T., and Bartle K.D., "A Comparison of the Particulate Composition between Turbocharged and Naturally Aspirated DI Diesel Engines,” SAE Technical Paper No. 910733, Warrendale, PA, 1991

47. Wachter W.F., "Analysis of Transient Emission Data of A Model Year 1991 Heavy Duty Diesel Engine," SAE Technical Paper No. 900443, Warrendale, PA, 1990

48. Kittelson D.B., Watts W.F., and Arnold M., "Review of Diesel Particulate Matter Sampling Methods: Supplemental Report \#1," University of Minnesota, Minneaplolist, MN, Jul, 1998

49. Schuetzle D., "Sampling of Vehicle Emissions of Chemical Analysis and Biological Testing," Environ Health Perspect, Jan, 1983, Vol. 47, pp: 65-80

50. Pierson W.R., and Brachaczek W.W., "Particulate Matter Associated with Vehicles on the Road," Aerosol Sci Tech, Jul, 1983, Vol. 2, No. 1, pp: 1-40

51. Rogge W.F., Hildemann L.M., Mazurek M.A., Cass G.R., and Simoneit B.R.T., "Sources of Fine Organic Aerosol. 2. Noncatalyst and Catalyst-Equipped Automobiles and Heavy-Duty Diesel Trucks," Environ Sci Technol, 1993, Vol. 27, pp: 636-651

52. Whitby K.T., and Cantrell B.K., "Atmospheric Aerosols - Characteristics and Measurement," ICESA Conference Proceedings, IEEE \#75-CH 1004-1 ICESA, Paper 29-1, pp: 6

53. Bagan J., "Study of Particle Size Distributions Emitted by A Diesel Engine," SAE Technical Paper No. 1999-01-1141, Warrendale, PA, 1999

54. Winer A.M., and Busby W.F., "Diesel Exhaust: A Critical Analysis of Emissions, Exposure, and Health Effects," Health Effects Institute, Cambridge, MA, 1995 
55. Ghio A.J., Richards J.H., and Dailey L.A., "The Effects of Oxidants on Modifying the Toxicity of Ambient PM," Society of Toxicology Annual Meeting, San Francisco, CA, 2000

56. Finlayson-Pitts B.J., and Pitts J.N., "Atmospheric Chemistry: Fundamentals and Experimental Techniques," John Wiley \& Sons, NY, 1986

57. Kamens R.M., Guo Z., and Fulcher J.N., "Influence of Humidity, Sunlight, and Temperature on the Daytime Decay of Polyaromatic Hydrocarbons on Atmospheric Soot Particles,” Environ Sci Technol, Jan, 1988, Vol. 22, No. 1, pp: 103-108

58. Weingartner E., Burtscher H., and Baltensperger U., "Hygroscopic Properties of Carbon and Diesel Soot Particles," Atmos Environ, Aug, 1997, Vol. 31, No. 15, pp: 2311-2327

59. "Diesel Exhaust: A Critical Analysis of Emissions, Exposure and Health Effects. A Special Report of Institute's Diesel Working Group," Health Effects Institute, Cambridge, MA, 1995

60. "Diesel Fuel and Exhaust Emissions - Environmental Health Criteria 171," World Health Organization, Geneva, Switzerland, 1996

61. "Air Quality Guidelines - Second Edition," WHO Regional Office for Europe, Copenhagen, Denmark, 2000

62. Schwartz J., Dockery D.W., and Neas L.M., "Is Daily Mortality Associated Specifically with Fine Particles?” J Air Waste Manag Assoc, Oct, 1996, Vol. 46, No. 10, pp: 927-939

63. Schwartz J., Slater D., Larson T.V., Pierson W.E., and Koenig J.Q., "Particulate Air Pollution and Hospital Emergency Room Visits for Asthma in Seattle," Am Rev Respir Dis, Apr, 1993, Vol. 147, No. 4, pp: 826-831

64. Stephen K., Van D.E., and Charles P.Q., "Particulate Air Pollution and Morbidity in the California Central Valley: A High Particulate Pollution Region - Final Report,” Kaiser Foundation Research Institute, Oakland, CA, 2002

65. Rudell B., Sandstrom T., Stjernberg N., and Kolmodin-Hedman B., "Controlled Diesel Exhaust Exposure in An Exposure Chamber: Pulmonary Effects Investigated with Bronchoalveolar Lavage," J Aerosol Sci, 1990, Vol. 21, Suppl. 1, pp: 411-414 
66. Rudell B., Sandström T., Hammarström U., Ledin M.L., Hörstedt P., and Stjernberg N., "Evaluation of An Exposure Setup for Studying Effects of Diesel Exhaust in Humans," Int Arch Occup Environ Health, 1994, Vol. 66, No. 2, pp: 7783

67. Rudell B., Ledin M.L., Hammarström U., Stjernberg N., and Sandström T., "Effects on Symptoms and Lung Function in Humans Experimentally Exposed to Diesel Exhaust,” Occup Environ Med, Oct, 1996, Vol. 53, No. 10, pp: 658-662

68. Kahn G., Orris P., and Weeks J., "Acute Overexposure to Diesel Exhaust: Report of 13 Cases," Am J Ind Med, 1988, Vol. 13, No. 3, pp: 405-406

69. Wade J.F. III., and Newman L.S., "Diesel Asthma: Reactive Airways Disease Following Overexposure to Locomotive Exhaust," J Occup Med, Feb, 1993, Vol. 35, No. 3, pp: 149-154

70. Reger R., Hancock J., Hankinson J., Hearl F., and Merchant J., "Coal Miners Exposed to Diesel Exhaust Emissions," Ann Occup Hyg, 1982, Vol. 26, No. 8, pp: $799-815$

71. Gamble J., Jones W., and Minshall S., "Epidemiological-Environmental Study of Diesel Bus Garage Workers: Chronic Effects of Diesel Exhaust on the Respiratory System,” Environ Res, Oct, 1987, Vol. 44, No. 1, pp: 6-17

72. Heinrich U., Peters L., Funcke W., Pott F., Mohr U., and Stöber W., "Investigation of Toxic and Carcinogenic Effects of Diesel Exhaust in Long-Term Inhalation Exposure of Rodents," Dev Toxicol Environ Sci, 1982, Vol. 10, pp: 225-242

73. Heinrich U., Muhle H., Takenaka S., Ernst H., Fuhst R., Mohr U., Pott F., and Stöber W., "Chronic Effects on the Respiratory Tract of Hamsters, Mice and Rats After Long-Term Inhalation of High Concentrations of Filtered and Unfiltered Diesel Engine Emissions,” J Appl Toxicol, Dec, 1986, Vol. 6, No. 6, pp: 383-395

74. Heinrich U., Fuhst R., Rittinghausen S., Creutzenberg O., Bellmann B., Koch W., and Levsen K., "Chronic Inhalation Exposure of Wistar Rats and Two Different Strains of Mice to Diesel Engine Exhaust, Carbon Black, and Titanium Dioxide," Inhal Toxicol, May, 1995, Vol. 7, No. 4, pp: 533-556 
75. Brightwell J., Fouillet X., Cassano-Zoppi A.L., Gatz R., and Duchosal F., "Neoplastic and Functional Changes in Rodents After Chronic Inhalation of Engine Exhaust Emissions,” Dev Toxicol Environ Sci, 1986, Vol. 13, pp: 471-485

76. Ishinishi N., Kuwabara N., Nagase S., Suzuki T., Ishiwata S., and Kohno T., "Long-Term Inhalation Studies of Diesel Exhaust on F344 SPF Rats," Dev Toxicol Environ Sci, 1986, Vol. 13, pp: 349-360

77. Murphy S.A.M., Berube K.A., and Richards R. J., "Bioreactivity of Carbon Black and Diesel Exhaust Particles to Primary Clara and Type II Epithelial Cell Cultures,” Occup Environ Med, Dec, 1999, Vol. 56, No. 12, pp: 813-819

78. Wichmann H.E., and Peters A., "Epidemiological Evidence of the Effects of Ultrafine Particle Exposure," Philosophical Transactions: Mathematical, Physical and Engineering Sciences, Ultrafine Particles in the Atmosphere, 2000, Vol. 358, No. 1775 , pp: 2751-2768

79. Renwick L.C., Donaldson K., and Clouter A., "Impairment of Alveolar Macrophage Phagocytosis by Ultrafine Particles," Toxicol Appl Pharmacol, Apr, 2001, Vol. 172, No. 2, pp: 119-127

80. Oberdorster G., and Sharp Z., "Extrapulmonary Translocation of Ultrafine Carbon Particles Following Whole-Body Inhalation Exposure of Rats," J Toxicol Environ Health A, Oct, 2002, Vol. 65, No. 20, pp: 1531-1543

81. Burtscher H., "Literature Study on Tailpipe Particulate Emission Measurement for Diesel Engines," University of Applied Science, Switzerland, 2001

82. Mohr M., and Lehmann U., "Comparison Study of Particle Measurement Systems for Future Type Approval Application," GRPE Particle Measurement Programme Research Report 202779, 2003

83. Mcmurry P.H., "The History of Condensation Nucleus Counters," Aerosol Sci Technol, Oct, 2000, Vol. 33, No. 4, pp: 297-322

84. Pui D.Y.H., and Swift D.L., "Direct-Reading Instruments for Airborne Particles," Ch.16 in "Air Sampling Instruments for Evaluation of Atmospheric Contaminants, 8th Edition," Ed. by Cohen B.S., Hering S.V., ACGIH, Cincinnati, OH, 1995, pp: $337-368$ 
85. Wang S.C., and Flagan R.C., "Scanning Electrical Mobility Spectrometer," Aerosol Sci Technol, Jan, 1990, Vol. 13, No. 2, pp: 230-240

86. Liu B.Y.H., and Pui D.Y.H., "On the Performance of the Electrical Aerosol Analyzer,” J Aerosol Sci, Jun, 1975, Vol. 6, No. 3-4, pp: 249-264

87. Patashnick H., and Rupprecht G., "Personal Dust Exposure Monitor Based on the Tapered Elemental Oscillating Microbalance," Bul Mines, OFR 56-84, NTIS PB 84-173749

88. Hillamo R.E., and Kauppinen E.I., "On the Performance of the Berner Low Pressure Impactor,” J Aerosol Sci, Jan, 1991, Vol. 14, No.1, pp: 33-47

89. Keskinen J., Pietarinen K., and Lehtimaki M., "Electrical Low Pressure Impactor," J Aerosol Sci, 1992, Vol. 23, No. 4, pp: 353-360

90. Faxvog F.R., and Roessler D.M., "Optoacoustic Measurements of Diesel Particulate Emissions," J Appl Phsy, Dec, 1979, Vol. 50, No. 12, pp: 7880-7882

91. Marple V.A., Rubow K.L., and Behm S.M., "A Microorifice Uniform Deposit Impactor (MOUDI): Description, Calibration, and Use,” J Aerosol Sci Technol, Jan, 1991, Vol. 14, No. 4, pp: 434-446

92. Marple V.A, Rubow K.L., and Olson B.A., "Low Pressure Stages for the Microorifice Uniform Deposit Impactor (MOUDI),” Ed. by Flagan R.C., 4th International Aerosol Conference Abstracts, 1994, Vol. 2, pp: 6

93. Burtscher H., and Siegmann H.C., "Photoemission for in Situ Analysis of Particulate Combustion Emissions," Water, Air, \& Soil Pol, 1993, Vol. 68, No. 1-2, pp: $125-136$

94. Silva P.J., and Prather K.A., "On-Line Characterization of Individual Particles from Automobile Emissions," Environ Sci Technol, Oct, 1997, Vol. 31, No. 11, pp: 3074-3080

95. Gard E., Mayer J.E., Morrical B.D., Dienes T., Fergenson D.P., and Prather K.A., "Realtime Analysis of Individual Atmospheric Aerosol Particles: Design and Performance of A Portable ATOFMS," Anal Chem, Oct, 1997, Vol. 69, No. 20, pp: 4083-4091 
96. Gaggeler H.W., Baltensperger U., Emmenegger M., Jost D.T., Schmidt-Ott A., Haller P., and Hofmann M., “The Epiphaniometer, A New Device for Continuous Aerosol Monitoring,” J Aerosol Sci, 1989, Vol. 20, No. 5, pp: 557-564

97. Maricq M., "Vehicle Particulate Emissions: A Comparison of ELPI, SMPS and Mass Measurement," Included in Presentation Handouts, Presented at International Seminar on Particle Size Distribution Measurement from Combustion Engines, Espoo, Finland, May 18-19, 1998

98. Andersson J.D., Brown A.J.M., Clarke D.P., Lance D.L., Mcgrath N.J., and Savill R.H., "UK Particle Measurement Programme - Heavyduty Methodology Development Final Report," Ricardo Consulting Engineers Ltd., DP 02/2493, Jul, 2002

99. Coen V.G., Jan C.M.M., Michiel M., and Jacob A.M., "The Choice of Instrument (ELPI And/Or SMPS) for Diesel Particulate Measurements," SAE Technical Paper No. 2003-01-0784, Warrendale, PA, 2003

100. Price P., Stone R., Collier T., Davies M., and Scheer V., "Dynamic Particulate Measurements from A DISI Vehicle: A Comparison of DMS500, ELPI, CPC and PASS," SAE Technical Paper No. 2006-01-107, Warrendale, PA, 2006

101. Morrow P.E., Bates D.V., and Fisher B.R., "Deposition and Retention Models for Internal Dosimetry of the Human Respiratory Tract (Report of the International Commission on Radiological Protection: ICRP: Task Group on Lung Dynamics),” Health Phys, Feb, 1966, Vol. 12, No. 2, pp: 173-207

102. Raabe O.G., "Deposition and Clearance of Inhaled Aerosols," U.S. Department of Energy, National Technical Information Service, UCD-472-503, Springfield, VA, 1979

103. Khalek I.A., Kittelson D.B., and Brear F., "Influence of Dilution Conditions on Diesel Exhaust Particle Size Distribution Measurements," SAE Technical Paper No. 1999-01-1142, Warrendale, PA, 1999

104. Dolan D.F., Kittelson D.B., and Whitby K.T., "Measurement of Diesel Exhaust Particle Size Distributions," ASME Paper 75-WA/APC-5 
105. Kittelson D.B., and Dolan D.F., "Diesel Exhaust Aerosols," in "Generation of Aerosols and Facilities for Exposure Experiments," Ed. by Willeke K., Ann Arbor Science, Ann Arbor, MI, pp: 337-359

106. Khalek I.A., and Kittelson D.B., "Diesel Trap Performance: Particle Size Measurements and Trends," SAE Technical Paper No. 982566, Warrendale, PA, 1998

107. Podsiadlik D.H., Chase R.E., and Lewis D., "Phase-Based TEOM Measurements Compared with Traditional Filters for Diesel PM," SAE Technical Paper No. 200301-0783, Warrendale, PA, 2003

108. Gray H.A., Cass G.R., Huntzicker J.J., Heyerdahl E.K., AND RAU J.A., "Characteristics of Atmospheric Organic and Elemental Carbon Particle Concentrations in Los Angeles," Environ Sci Technol, Jun, 1986,Vol. 20, No. 6, pp: 580-589

109. Cass G.R., and Gray H.A., "Regional Emissions and Atmospheric Concentrations of Diesel Engine Particulate Matter: Los Angeles as A Case Study," in "Diesel Exhaust: A Critical Analysis of Emissions, Exposure, and Health Effects - A Special Report of the Institute's Diesel Working Group," Health Effects Institute, Cambridge, MA, 1995, pp: 125-137

110. Maricq M.M., Chase R.E., and Podsiadlik D.H., "Vehicle Exhaust Particle Size Distributions: A Comparison of Tailpipe and Dilution Tunnel Measurements," SAE Technical Paper No. 1999-01-1461, Warrendale, PA, 1999

111. Kinney P.L., Aggarwal M., Northridge M.E., Janssen N.A.H., and Shepard P., "Airborne Concentration of PM2.5 and Diesel Exhaust Particles on Harlem Sidewalks: A Community-Based Pilot Study," Environ Health Perspect, Mar, 2000, Vol. 108, No. 3, pp: 213-218

112. Vogt R., and Scheer V., "Nanoparticle Formation in Diesel Vehicle Exhaust: A Comparison of Laboratory and Chasing Experiments," Presented at the 5th International Workshop on Nanoparticle Measurements, Zurich, Switzerland, Aug 6-8, 2001

113. Gertler A.W., Gillies J.A., Pierson W.R., Rogers C.F., Sagebiel J.C., Abu-Allaban M., Coulombe W., Tarnay L., and Cahill T.A., "Real-World Particulate Matter and 
Gaseous Emissions from Motor Vehicles in A Highway Tunnel," in "Emissions from Diesel and Gasoline Engines Measured in Highway Tunnels - Research Report,” Res Rep Health Eff Inst, Jan, 2002, Vol. 10, No. 107, pp: 5-56

114. Hasegawa S., Hirabayashi M., Kobayashi S., Moriguchi Y., Konda Y., Tanabe K., and Wakamatsu S., "Size Distribution and Characterization of Ultrafine Particle in Roadside Atmosphere," J Environ Sci Health A Tox Hazard Subst Environ Eng, Jan, 2004, Vol. 39, No. 10, pp: 2671-2690

115. Bernemyr H., and Angström H.E., "Characterization of Tailpipe Exhaust Particles using A Rotating Disc Diluter and A Volatility Tandem DMA (V-TDMA)," SAE Technical Paper No. 2006-01-3367, Warrendale, PA, 2006

116. Anderson W.C., "Research Needs Related to Improving Air Emissions from Diesel Engines, Gas Turbines, and Ordnance," American Academy of Environmental Engineers, Annapolis, MD, 1998

117. Kim D.H., "Nucleation and Coagulation of Particulate Matter Inside A Turbulent Exhaust Plume of A Diesel Vehicle," Doctoral Dissertation, West Virginia University, Department of Mechanical and Aerospace Engineering, Morgantown, WV, 2002

118. Barad M.L., "Project Prairie Grass, A Field Program in Diffusion," Geophysical Research Paper, Report No. AFCRC-TR-58-235, Air Force Cambridge Research Center, Bedford, MA, 1958

119. Biltoft C.A., "Customer Report for Mock Urban Setting Test," Report WDTC-FR01-121, US Army Dugway Proving Ground, Dugway, UT, 2001

120. Hanna S.R., "Concentration Fluctuations in A Smoke Plume," Atmos Environ, Jan, 1984, Vol. 18, No. 6, pp: 1091-1106

121. Gautam M., Xu Z., Ayala A., and Mehta S., "Diesel Exhaust Plume Studies: Wind Tunnel Experiments and Modeling," 4th ETH Nanoparticle Measurement Workshop, Zurich, Switzerland, Aug 7-9, 2000

122. Kleeman M.J., and Cass G.R., "Source Contributions to the Size and Composition Distribution of Urban Particulate Air Pollution," Atmos Environ, Aug, 1998, Vol. 32, No. 16, pp: 2803-2816 
123. Hyvönen S., Junninen H., Laakso L., Dal Maso M., Grönholm T., Bonn B., Peronen P., Aalto P., Hiltunen V., Pohja T., Launiainen S., Hari P., Mannila H., and Kulmala M., "A Look at Aerosol Formation using Data Mining Techniques," Atmos Chem Phys Discuss, 2005, Vol. 5, pp: 7577-7611

124. Wei T., Alan H., Brain B., and Walter S., "Application of CFD Simulations for Short-Range Atmospheric Dispersion Over Open Fields and within Arrays of Buildings," AMS 14th Joint Conference on the Applications of Air Pollution Meteorology with the A\&WMA, Atlanta, GA, Jan 30 - Feb 2, 2006

125. Hanna S.R., Brown M.J., Camelli F.E., Chan S.T., Coirier W.J., Hansen O.R., Huber A.H., Kim S., and Reynolds R.M., "Detailed Simulations of Atmospheric Flow and Dispersion in Urban Downtown Areas by Computational Fluid Dynamics (CFD) Models - An Application of Five CFD Models to Manhattan," Bulletin of the American Meteorological Society, 2006, Paper UCRL-JRNL-219591

126. Kim D.H., Gautam M., and Gera D., "Modeling Nucleation and Coagulation Models in the Formation of Particulate Matter Inside A Turbulent Exhaust Plume of A Diesel Engine,” J Colloid Interface Sci, 2002, Vol. 249, pp: 96-103

127. Jiang P.Z., Lignell D.O., Kelly K.E., Lighty J.S., and Sarofim A.F., "Simulation of the Evolution of Particle Size Distribution in A Vehicle Exhaust Plume with Unconfined Dilution by Ambient Air,” J Air Waste Manag Assoc, Apr, 2005, Vol. 55, No. 4, pp: 437-445

128. Uhrner U., Von Löwis S., Vehkamäki H., Wehner B., Bräsel S., Hermann M., Stratmann F., Kulmala M., and Wiedensohler A., "Dilution and Aerosol Dynamics within A Diesel Car Exhaust Plume - CFD Simulations of On-Road Measurement Conditions," Atmos Environ, Nov, 2007, Vol. 41, No. 35, pp: 7440-7461

129. Collings N., Reavell K.S., and Hands T., "A Fast Response Particulate Spectrometer for Combustion Aerosols," SAE Technical Paper No. 2002-01-2714, Warrendale, PA, 2002

130. Reavell K.S., Hands T., and Collings N., "Determination of Real Time Particulate Size Spectra and Emission Parameters with A Differential Mobility Spectrometer," 6th International ETH-Conference on Nanoparticle Measurement, Zurich, Switzerland, Aug, 2002 
131. Collings N., Reavell K.S., Hands T., and Tate J., "Roadside Aerosol Measurements with A Fast Particulate Spectrometer,” JSAE Technical Paper No. 20035407, 2003

132. Kittelson D., Hands T., Nickolaus C., Collings N., Niemela V., and Twigg M.T., "Mass Correlation of Engine Emissions with Spectral Instruments," 10th Diesel Engine Emissions Reduction (DEER) Workshop, Coronado, CA, Aug 29 - Sept 2, 2004

133. Clark N., Tatli E., Barnett N., Wayne W.S., and Mckain D.L., "Characterization and Abatement of Diesel Crankcase Emissions," SAE Technical Paper No. 200601-3372, Warrendale, PA, 2006

134. Buzzard N., Clark N., and Guffey S., "Investigation into Pedestrian Exposure to Near-Vehicle Exhaust Emissions,” Environ Health, Mar, 2009, Vol. 8, No. 13

135. http://www.cambustion.com/prodocts/dms500

136. “Semtech PPMD User Manual," Sensors Inc., Jan, 2006

137. “Automotive Emission Analyzer System MEXA-7200 Manual,” Horiba Ltd., Apr, 2007

138. Bugarski A.D., "Characterization of Particulate Matter and Hydrocarbon Emissions from In-Use Heavy-Duty Diesel Engines," Doctoral Dissertation, Department of Mechanical and Aerospace Engineering, West Virginia University, Morgantown, WV, 1999

139. "Laminar Flow Elements Installation \& Operation Instructions," Meriam Instrument, File No. 501:440-10

140. Wu Y., Carder D., Shade B., Atkinson R., Clark N., and Gautam M., “A CFR1065Compliant Transportable/On-Road Low Emissions Measurement Laboratory with Dual Primary Full-Flow Dilution Tunnels," Proceedings of ASME Internal Combustion Engine Division 2009 Spring Technical Conference, ICES200976090, May, 2009

141. Wu Y., Clark N., Carder D., Thompson G., Gautam M., and Lyons D., "Parametric Study of 2007 Standard Heavy Duty Diesel Engine Particulate Matter Sampling System,” SAE Technical Paper No. 2007-01-0060, Warrendale, PA, 2007 
142. Engeljehringer F., Schindler W., and Sulzer R., "Meeting ISO 8178 Requirements for the Measurement of Diesel Particulates with Partial-Flow Dilution Systems," SAE Technical Paper No. 932466, Warrendale, PA, 1993

143. Wayne W.S., Corrigan E., Atkinson R., Clark N., and Lyons D., "Measuring Diesel Emissions with A Split Exhaust Configuration," SAE Technical Paper No. 200101-1949, Warrendale, PA, 2001

144. Ayala A., Chernich D., Huai T., Johnson K., and Miller J., "Emissions Correlation between A Partial-Flow Diluter and the Full-Flow Constant Volume Sampler (CVS) for A Heavy-Duty Vehicle Under Steady-State Operation," SAE Technical Paper No. 2005-01-3798, Warrendale, PA, 2005

145. Wei Q., Porter S., Harvey N., Kihara N., and Khalek I., "Diesel Exhaust Particulate Sampler for On-Board PM Measurement," SAE Technical Paper No. 2008-011180, Warrendale, PA, 2008

146. Bata R., Clark N., Gautam M., Howell A., Long T., Loth J., Lyons D., Palmer M., Smith J., and Wang W., "The First Transportable Heavy Duty Vehicle Emissions Testing Laboratory,” SAE Technical Paper No. 912668, Warrendale, PA, 1991

147. Ferguson D., Gautam M., Wang W., Clark N., Lyons D., Bata R., Palmer G., and Katragada S., "Exhaust Emissions from In-Use Heavy Duty Vehicles Tested on A Transportable Transient Chassis Dynamometer," SAE Technical Paper No. 922436, Warrendale, PA, 1992

148. http://www.mathworks.com/products/simulink

149. Kittelson D.B., Arnold M., and Watts W.F., "Review of Diesel Particulate Matter Sampling Methods: Supplemental Report \#2," University of Minnesota, Minneaplolist, MN, Jul, 1998

150. Kittelson D.B., and Khalek I.A., "Formation of Nanoparticles During Exhaust Dilution," EFI Members Conference: "Fuels, Lubricants Engines, \& Emissions," Jan, 1999

151. Adrian B., and Allan D.K., "Heat Transfer Handbook," John Wiley \& Sons, NJ, 2003

152. Zhao Z.N., "Heat Transfer," Higher Education Press, Beijing, China, 2002 
153. Kays W.M., and Perkins H.C., "Forced Convection, Internal Flow in Ducts Handbook of Heat Transfer,” Mcgraw-Hill, NY, 1985, Sec. 7, pp: 1-193

154. Gnielinski V., "New Equations for Heat and Mass Transfer in Turbulent Pipe and Channel Flows,” Int Chem Eng, 1976, Vol. 16, pp: 359-368

155. Petukhov B.S., "Heat Transfer and Friction in Turbulent Pipe Flow with Variable Physical Properties," in "Advances in Heat Transfer," Academy Press, NY, 1970, Vol. 6, pp: 504-564

156. Churchill S.W., and Chu H.H.S., "Correlating Equations for Laminar and Turbulent Free Convection from A Horizontal Cylinder,” Int J Heat Mass Transfer, 1975, Vol. 18, pp: 1323

157. Ayala A., Olson B., and Cantrell B., "Working Quality Assurance Plan for Diesel Aerosol Sampling Methodology (CEC Project E-43), Final Report, CRC Project E43-3," Feb, 2002

158. Ayala A., Olson B., Cantrell B., Drayton M., and Barsic N., "Estimation of Diffusion Losses when Sampling Diesel Aerosol: A Quality Assurance Measure,” SAE Technical Paper No. 2003-01-1896, Warrendale, PA, 2003

159. Clark N.N., Atkinson C.M., Mckain D.L., and Nine R.D., "Speciation of Hydrocarbon Emissions from A Medium Duty Diesel Engine," SAE Technical Paper No. 960322, Warrendale, PA, 1996

160. Gilbert M.S., and Clark N.N., "Measurement of Particulate Matter from Diesel Engine Exhaust using A Tapered Element Oscillating Microbalance,” International Journal of Engine Research, 2001, Vol. 2, No. 4, pp: 277-287

161. Gilbert M.S., "Investigation into the Use of A Tapered Element Oscillating Microbalance for Real-Time Particulate Measurement," Master's Thesis, Department of Mechanical and Aerospace Engineering, West Virginia University, Morgantown, WV, 2002

162. Wang W.G., Lyons D.W., Clark N.N., and Gautam M., "Emissions from Nine Heavy Trucks Fueled by Diesel and Biodiesel Blend without Engine Modification," Environ Sci Technol, Feb, 2000, Vol. 34, No. 6, pp: 933-939 
163. Jarrett R., and Clark N.N., "Evaluation of Methods for Determining Continuous Particulate Matter from Transient Testing of Heavy Duty Diesel Engines" SAE Technical Paper No. 2001-01-3575, Warrendale, PA, 2001

164. Wang W., Bata R., Lyons D., Clark N., Palmer M., Gautam M., Howell A., and Rapp B., "Transient Response in A Dynamometer Power Absorption System," SAE Technical Paper No. 920252, Warrendale, PA, 1992

165. Wang W., Palmer M., Bata M., Clark N., Gautam M., and Lyons D., "Determination of Heavy Duty Vehicle Energy Consumption by A Chassis Dynamometer," SAE Technical Paper No. 922435, Warrendale, PA, 1992

166. Clark N., Gadapati C., Kelly K., White C., Lyons D., Wang W., Gautam M., and Bata R., "Comparative Emissions from Natural Gas and Diesel Buses," SAE Technical Paper No. 952746, Warrendale, PA, 1995

167. Walkowicz K., Proc K., Wayne W.S., Nine R., Campbell K., and Wiedemeier G., "Chassis Dynamometer Emission Measurements from Refuse Trucks using DualFuel $^{\text {TM }}$ Natural Gas Engines," SAE Technical Paper No. 2003-01-3366, Warrendale, PA, 2003

168. Clark N.N., Khan A.S., Wayne W.S., Gautam M., Thompson G., Mckain D., Lyons D., and Barnett R., "Weight Effect on Emissions and Fuel Consumption from Diesel and Lean-Burn Natural Gas Transit Buses," SAE Technical Paper No. 200701-3626, Warrendale, PA, 2007

169. Vallaisamy R., Clark N., Thompson G., Atkinson R., Tissera C., and Swartz M., "Assessment of Nox Destruction in Diesel Engines by Injecting NO in the Intake Manifold," SAE Technical Paper No. 2005-01-0370, Warrendale, PA, 2005

170. Tissera C., Swartz M., Tatli E., Vellaisamy R., Clark N., Thompson G., and Atkinson R., "Nox Decomposition in Natural Gas, Diesel and Gasoline Engines for Selective Nox Recirculation," SAE Technical Paper No. 2005-01-2144, Warrendale, PA, 2005

171. Stetter J., Foster D., and Schauer J., "Modern Diesel Particulate Matter Measurements and the Application of Lessons Learned to 2007 Levels and Beyond," SAE Technical Paper No. 2005-01-0194, Warrendale, PA, 2005 
172. May J., Bosteels D., Such C., Nicol A., and Andersson J., "Heavy-Duty Engine Particulate Emissions: Application of PMP Methodology to Measure Particle Number and Particulate Mass," SAE Technical Paper No. 2008-01-1176, Warrendale, PA, 2008

173. Conover W.J., "Practical Nonparametric Statistics - Third Edition," John Willey \& Sons, NY, 1999, pp: 456-465

174. Hogg R.B., Craig A., and Mckean J.W., "Introduction to Mathematical Statistics, 6th Edition," Prentice Hall, NJ, 2004

175. Khalek I., Kittelson D., and Brear F., "Nanoparticle Growth During Dilution and Cooling of Diesel Exhaust: Experimental Investigation and Theoretical Assessment," SAE Technical Paper No. 2000-01-0515, Warrendale, PA, 2000

176. Symonds J.P.R., Reavell K.S.J., Olfert J.S., Campbell B.W., and Swift S.J., "Diesel Soot Mass Calculation in Real-Time with A Differential Mobility Spectrometer," J Aerosol Sci, 2007, Vol. 38, No. 1, pp: 52-68

177. "Guide to the Expression of Uncertainty in Measurement," International Organization for Standardization, Geneva, Switzerland, 1995

178. "Particulate Mass Measurement with DMS Series Fast Spectrometers," Cambustion Application Note DMS01 


\section{Appendix}

Table A1 Chemical composition and Thermodynamic property of engine exhaust and dry air (Pressure at 0.1MPa)

\begin{tabular}{|c|c|c|c|}
\hline \multirow[b]{3}{*}{$\begin{array}{c}\text { Chemical } \\
\text { Compositio } \\
\text { n }\end{array}$} & \multicolumn{3}{|c|}{ Mass Fraction (\%) } \\
\hline & & \multicolumn{2}{|c|}{$\begin{array}{c}\text { Exhaust Gas of Different Air Fuel Ratio A/F } \\
\text { (Diesel Fuel: } C_{12.5} \mathbf{H}_{22.2} \text { ) }\end{array}$} \\
\hline & Dry Air & $A / F=16$ & $A / F=100$ \\
\hline $\mathrm{N} 2$ & 75.47 & 71.31 & 74.80 \\
\hline $\mathrm{O2}$ & 23.20 & 2.18 & 19.84 \\
\hline Ar & 1.29 & 1.21 & 1.28 \\
\hline $\mathrm{CO}_{2}$ & 0.04 & 18.50 & 2.99 \\
\hline H2O & 0.00 & 6.81 & 1.09 \\
\hline $\begin{array}{c}\text { Mole } \\
\text { Weight }\end{array}$ & 28.995 & 29.056 & 29.005 \\
\hline
\end{tabular}

Thermodynamic Properties (specific heat, viscosity, conductivity)

\begin{tabular}{|c|c|c|c|c|c|c|c|c|c|}
\hline \multirow[b]{2}{*}{$\begin{array}{c}\text { Gas } \\
\text { Temperatur } \\
\text { e } \\
\left({ }^{\circ} \mathrm{C}\right) \\
\end{array}$} & \multicolumn{3}{|l|}{ Dry Air } & \multicolumn{3}{|c|}{$A / F=16$} & \multicolumn{3}{|c|}{$A / F=100$} \\
\hline & $\begin{array}{c}\mathrm{kJ} /(\mathrm{Kg} \cdot \mathrm{K}) \\
]\end{array}$ & $\left(\mathrm{m}^{2} / \mathrm{s}\right)$ & $\begin{array}{c}{[\mathbf{W} /(\mathbf{m} \cdot \mathbf{K})} \\
]\end{array}$ & $\begin{array}{c}\mathrm{kJ} /(\mathrm{Kg} \cdot \mathrm{K}) \\
]\end{array}$ & $\left(\mathrm{m}^{2} / \mathrm{s}\right)$ & $\begin{array}{c}{[\mathbf{W} /(\mathbf{m} \cdot \mathbf{K})} \\
]\end{array}$ & $\begin{array}{c}{[\mathrm{kJ} /(\mathrm{Kg} \cdot \mathrm{K})} \\
]\end{array}$ & $\left(\mathrm{m}^{2} / \mathrm{s}\right)$ & $\begin{array}{c}{[\mathbf{W} /(\mathbf{m} \cdot \mathbf{K})} \\
]\end{array}$ \\
\hline 50 & 1.007 & 18.16 & 2.81 & 1.140 & 16.46 & 2.54 & 1.028 & 17.89 & 2.77 \\
\hline 75 & 1.009 & 20.71 & 2.99 & 1.145 & 18.68 & 2.71 & 1.031 & 20.39 & 2.95 \\
\hline 100 & 1.011 & 23.38 & 3.17 & 1.150 & 21.15 & 2.89 & 1.033 & 23.02 & 3.13 \\
\hline 125 & 1.014 & 26.20 & 3.35 & 1.156 & 23.74 & 3.06 & 1.037 & 25.81 & 3.30 \\
\hline 150 & 1.017 & 29.12 & 3.52 & 1.162 & 26.41 & 3.23 & 1.040 & 28.69 & 3.47 \\
\hline 175 & 1.021 & 32.15 & 3.69 & 1.169 & 29.21 & 3.39 & 1.045 & 31.68 & 3.64 \\
\hline 200 & 1.025 & 35.28 & 3.86 & 1.175 & 32.12 & 3.56 & 1.049 & 34.77 & 3.81 \\
\hline 225 & 1.030 & 38.52 & 4.02 & 1.183 & 35.11 & 3.72 & 1.055 & 37.97 & 3.97 \\
\hline 250 & 1.035 & 41.85 & 4.18 & 1.191 & 38.21 & 3.87 & 1.060 & 41.27 & 4.13 \\
\hline 275 & 1.040 & 45.27 & 4.34 & 1.199 & 41.39 & 4.03 & 1.065 & 44.65 & 4.29 \\
\hline 300 & 1.045 & 48.79 & 4.50 & 1.206 & 44.66 & 4.18 & 1.071 & 48.13 & 4.45 \\
\hline 325 & 1.051 & 52.40 & 4.65 & 1.215 & 48.04 & 4.33 & 1.077 & 51.70 & 4.60 \\
\hline 350 & 1.057 & 56.10 & 4.80 & 1.223 & 51.47 & 4.48 & 1.084 & 55.36 & 4.75 \\
\hline 375 & 1.063 & 59.89 & 4.94 & 1.232 & 55.01 & 4.63 & 1.090 & 59.11 & 4.89 \\
\hline 400 & 1.069 & 63.79 & 5.08 & 1.239 & 58.62 & 4.77 & 1.096 & 62.96 & 5.03 \\
\hline 425 & 1.075 & 67.78 & 5.22 & 1.247 & 62.32 & 4.91 & 1.103 & 66.91 & 5.17 \\
\hline 450 & 1.081 & 71.87 & 5.36 & 1.255 & 66.12 & 5.06 & 1.109 & 70.95 & 5.31 \\
\hline 475 & 1.087 & 76.04 & 5.49 & 1.264 & 69.99 & 5.20 & 1.115 & 75.07 & 5.44 \\
\hline 500 & 1.093 & 80.28 & 5.63 & 1.272 & 73.93 & 5.34 & 1.122 & 79.26 & 5.58 \\
\hline 525 & 1.098 & 84.59 & 5.76 & 1.280 & 77.95 & 5.48 & 1.127 & 83.53 & 5.72 \\
\hline 550 & 1.104 & 88.98 & 5.89 & 1.288 & 82.05 & 5.61 & 1.133 & 87.87 & 5.85 \\
\hline 575 & 1.110 & 93.44 & 6.02 & 1.295 & 86.22 & 5.75 & 1.140 & 92.28 & 5.98 \\
\hline 600 & 1.115 & 97.95 & 6.15 & 1.301 & 90.46 & 5.89 & 1.145 & 96.75 & 6.11 \\
\hline
\end{tabular}


Table A2 Summary of particle size distributions for the comparison study

\begin{tabular}{|c|c|}
\hline $\begin{array}{c}\text { number } \\
\text { label }\end{array}$ & Particle size distribution description \\
\hline 1 to 7 & $\begin{array}{l}\text { Measured at outlet of secondary dilution tunnel with secondary flow rate of } 2.0 \text { SCFM (56.6 } \\
\text { lpm), corresponding dilution ratios } 1,1.14,1.33,1.6,2,4 \text {, and } 8 \text {. (engine running at } 102 \mathrm{Nm} \text { ) }\end{array}$ \\
\hline 8 to 14 & $\begin{array}{l}\text { Measured at outlet of secondary dilution tunnel with secondary flow rate of } 2.25 \text { SCFM (63.7 } \\
\text { lpm), corresponding dilution ratios } 1,1.13,1.29,1.5,1.8,3 \text {, and } 9 . \text { (engine running at } 102 \mathrm{Nm})\end{array}$ \\
\hline 15 to 21 & $\begin{array}{l}\text { Measured at outlet of secondary dilution tunnel with secondary flow rate of } 2 \text { SCFM (56.6 lpm), } \\
\text { corresponding dilution ratios } 1,1.14,1.33,2,4,8 \text {. (engine running at } 122 \mathrm{Nm})\end{array}$ \\
\hline 22 to 28 & $\begin{array}{l}\text { Measured at outlet of secondary dilution tunnel with total flow rate of } 2.25 \text { SCFM (63.7 lpm), } \\
\text { corresponding dilution ratios } 1,1.13,1.29,1.5,1.8,3 \text {, and } 9 \text { (engine running at } 102 \mathrm{Nm})\end{array}$ \\
\hline 29 to 42 & $\begin{array}{l}\text { Measured at P2 of primary tunnel with the primary flow rate } 800 \mathrm{SCFM}\left(0.378 \mathrm{~m}^{3} / \mathrm{s}\right) \text {, distances } \\
\text { to section center and corresponding dilution ratios are: } 0.0 \mathrm{~cm}, \mathrm{dr}=11 ; 1.3 \mathrm{~cm}, \mathrm{dr}=12 ; \\
2.5 \mathrm{~cm}, \mathrm{dr}=14 ; 3.8 \mathrm{~cm}, \mathrm{dr}=15.5 ; 5.1 \mathrm{~cm}, \mathrm{dr}=17 ; 6.4 \mathrm{~cm}, \mathrm{dr}=19 ; 7.6 \mathrm{~cm}, \mathrm{dr}=22 ; 8.6 \mathrm{~cm}, \mathrm{dr}=22 ; \\
12.2 \mathrm{~cm}, \mathrm{dr}=22 ; 15.0 \mathrm{~cm}, \mathrm{dr}=21 ; 17.3 \mathrm{~cm}, \mathrm{dr}=19 ; 19.3 \mathrm{~cm}, \mathrm{dr}=19 ; 21.1 \mathrm{~cm}, \mathrm{dr}=19 ; 22.9 \mathrm{~cm} \text {, } \\
\mathrm{dr}=19 \text { (engine running at } 102 \mathrm{Nm})\end{array}$ \\
\hline 43 to 57 & $\begin{array}{l}\text { Measured at P1 of primary tunnel with the primary flow rate } 800 \mathrm{SCFM}\left(0.378 \mathrm{~m}^{3} / \mathrm{s}\right) \text {, distances } \\
\text { to section center and corresponding dilution ratios are: } 0.0 \mathrm{~cm}, \mathrm{dr}=2.5 ; 1.3 \mathrm{~cm}, \mathrm{dr}=4 ; 2.5 \mathrm{~cm} \text {, } \\
\mathrm{dr}=8 ; 3.8 \mathrm{~cm}, \mathrm{dr}=42 ; 5.1 \mathrm{~cm}, \mathrm{dr}=54 ; 6.4 \mathrm{~cm}, \mathrm{dr}=48 ; 7.6 \mathrm{~cm}, \mathrm{dr}=40 ; 8.6 \mathrm{~cm}, \mathrm{dr}=33 ; 10.2 \mathrm{~cm} \text {, } \\
\mathrm{dr}=28 ; 12.2 \mathrm{~cm}, \mathrm{dr}=21 ; 15.0 \mathrm{~cm}, \mathrm{dr}=20 ; 17.3 \mathrm{~cm}, \mathrm{dr}=19 ; 19.3 \mathrm{~cm}, \mathrm{dr}=19 ; 21.1 \mathrm{~cm}, \mathrm{dr}=18 ; \\
22.9 \mathrm{~cm}, \mathrm{dr}=18 \text { (engine running at } 102 \mathrm{Nm} \text { ) }\end{array}$ \\
\hline 58 to 69 & $\begin{array}{l}\text { Measured at P2 of primary tunnel with the primary flow rate } 2000 \mathrm{SCFM}\left(0.944 \mathrm{~m}^{3} / \mathrm{s}\right) \text {, } \\
\text { distances to section center and corresponding dilution ratios are: } 0.0 \mathrm{~cm}, \mathrm{dr}=22 ; 1.3 \mathrm{~cm}, \mathrm{dr}=23 \text {; } \\
2.5 \mathrm{~cm}, \mathrm{dr}=25 ; 1.5 ", \mathrm{dr}=36 ; 6.4 \mathrm{~cm}, \mathrm{dr}=50 ; 8.6 \mathrm{~cm}, \mathrm{dr}=54 ; 12.2 \mathrm{~cm}, \mathrm{dr}=50 ; 15.0 \mathrm{~cm}, \mathrm{dr}=48 ; 22.9 \mathrm{~cm} \text {, } \\
\mathrm{dr}=44 ; 19.3 \mathrm{~cm}, \mathrm{dr}=45 ; 21.1 \mathrm{~cm}, \mathrm{dr}=44 ; 22.9 \mathrm{~cm}, \mathrm{dr}=44 \text { (engine running at } 102 \mathrm{Nm} \text { ) }\end{array}$ \\
\hline 70 to 82 & $\begin{array}{l}\text { Measured at } \mathrm{P} 1 \text { of primary tunnel with the primary flow rate } 2000 \mathrm{SCFM}\left(0.944 \mathrm{~m}^{3} / \mathrm{s}\right) \text {, } \\
\text { distances to section center and corresponding dilution ratios are: } 0.0 \mathrm{~cm}, \mathrm{dr}=5.5 ; 1.3 \mathrm{~cm}, \mathrm{dr}=11 ; \\
2.5 \mathrm{~cm}, \mathrm{dr}=28 ; 3.8 \mathrm{~cm}^{\prime}, \mathrm{dr}=70 ; 5.1 \mathrm{~cm}, \mathrm{dr}=120 ; 7.6 \mathrm{~cm}, \mathrm{dr}=88 ; 10.2 \mathrm{~cm}, \mathrm{dr}=66 ; 12.2 \mathrm{~cm}, \mathrm{dr}=46 ; \\
15.0 \mathrm{~cm}, \mathrm{dr}=44 ; 22.9 \mathrm{~cm}, \mathrm{dr}=41 ; 19.3 \mathrm{~cm}, \mathrm{dr}=42 ; 21.1 \mathrm{~cm}, \mathrm{dr}=41 ; 22.9 \mathrm{~cm}, \mathrm{dr}=41 \text { (engine running } \\
\text { at } 122 \mathrm{Nm})\end{array}$ \\
\hline 83 to 92 & $\begin{array}{l}\text { Measured at P2 of primary tunnel with the primary flow rate } 800 \mathrm{SCFM} \text {, distances to section } \\
\text { center and corresponding dilution ratios are: } 0.0 \mathrm{~cm}, \mathrm{dr}=12 ; 1.3 \mathrm{~cm}, \mathrm{dr}=14 ; 2.5 \mathrm{~cm}, \mathrm{dr}=15 \text {; } \\
3.8 \mathrm{~cm}, \mathrm{dr}=16 ; 5.1 \mathrm{~cm}, \mathrm{dr}=17 ; 6.4 \mathrm{~cm}, \mathrm{dr}=18 ; 8.6 \mathrm{~cm}, \mathrm{dr}=19 ; 12.2 \mathrm{~cm}, \mathrm{dr}=19 ; 15.0 \mathrm{~cm}, \mathrm{dr}=18 ; 9 " \text {, } \\
\mathrm{dr}=18 \text { (engine running at } 122 \mathrm{Nm} \text { ) }\end{array}$ \\
\hline
\end{tabular}

to be continued 
Table A2 continued

\begin{tabular}{|c|c|}
\hline 93 to 107 & 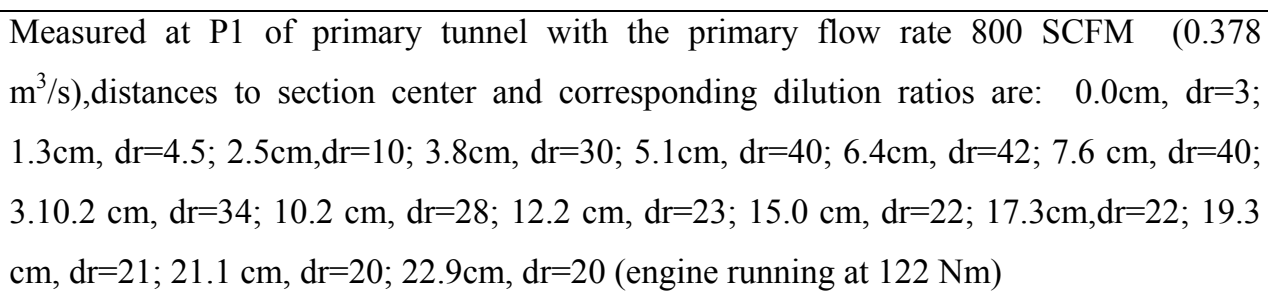 \\
\hline 108 to 115 & $\begin{array}{l}\text { Measured at P2 of primary tunnel with the primary flow rate } 2000 \mathrm{SCFM}\left(0.944 \mathrm{~m}^{3} / \mathrm{s} \text { ), }\right. \\
\text { distances to section center and corresponding dilution ratios are: } 0.0 \mathrm{~cm}, \mathrm{dr}=24 ; 1.3 \mathrm{~cm} \text {, } \\
\mathrm{dr}=26 ; 2.5 \mathrm{~cm}, \mathrm{dr}=30 ; 3.8 \mathrm{~cm}, \mathrm{dr}=38 ; 6.4 \mathrm{~cm}, \mathrm{dr}=48 ; 8.6 \mathrm{~cm}, \mathrm{dr}=52 ; 12.2 \mathrm{~cm}, \mathrm{dr}=44 ; 9 \text {, } \\
\mathrm{dr}=43 \text { (engine running at } 122 \mathrm{Nm} \text { ) }\end{array}$ \\
\hline 116 to 128 & $\begin{array}{l}\text { Measured at P1 of primary tunnel with the primary flow rate } 2000 \mathrm{SCFM}\left(0.944 \mathrm{~m}^{3} / \mathrm{s}\right) \text {, } \\
\text { distances to section center and corresponding dilution ratios are: } 0.0 \mathrm{~cm}, \mathrm{dr}=6 ; 1.3 \mathrm{~cm} \text {, } \\
\mathrm{dr}=11 ; 2.5 \mathrm{~cm}, \mathrm{dr}=30 ; 3.8 \mathrm{~cm}, \mathrm{dr}=57 ; 5.1 \mathrm{~cm}, \mathrm{dr}=114 ; 7.6 \mathrm{~cm}, \mathrm{dr}=101 ; 8.6 \mathrm{~cm}, \mathrm{dr}=85 ; \\
12.2 \mathrm{~cm}, \mathrm{dr}=67 ; 14.9 \mathrm{~cm}, \mathrm{dr}=48 ; 17.3 \mathrm{~cm}, \mathrm{dr}=45 ; 19.3 \mathrm{~cm}, \mathrm{dr}=44 ; 21.1 \mathrm{~cm}, \mathrm{dr}=43 ; 9 ", \mathrm{dr}=42 \\
\text { (engine running at } 122 \mathrm{Nm})\end{array}$ \\
\hline 129 to 138 & $\begin{array}{l}\text { Measured at P3 and P4 of primary tunnel and corresponding locations and primary flow } \\
\text { rates are:P3 - } 800 \mathrm{SCFM}\left(0.378 \mathrm{~m}^{3} / \mathrm{s}\right) ; \mathrm{P} 4-800 \mathrm{SCFM}\left(0.378 \mathrm{~m}^{3} / \mathrm{s}\right) ; \mathrm{P} 3-1200 \mathrm{SCFM} \\
\left(0.755 \mathrm{~m}^{3} / \mathrm{s}\right) ; \mathrm{P} 4-1200 \mathrm{SCFM}\left(0.755 \mathrm{~m}^{3} / \mathrm{s}\right) ; \mathrm{P} 3-1600 \mathrm{SCFM}\left(0.756 \mathrm{~m}^{3} / \mathrm{s}\right) ; \mathrm{P} 4-1600 \\
\mathrm{SCFM}\left(0.756 \mathrm{~m}^{3} / \mathrm{s}\right) ; \mathrm{P} 3-2000 \mathrm{SCFM}\left(0.944 \mathrm{~m}^{3} / \mathrm{s}\right) ; \mathrm{P} 4-2000 \mathrm{SCFM}\left(0.944 \mathrm{~m}^{3} / \mathrm{s}\right) ; \mathrm{P} 3- \\
\left.2500 \mathrm{SCFM}\left(1.180 \mathrm{~m}^{3} / \mathrm{s}\right) ; \mathrm{P} 4-2500 \mathrm{SCFM}\left(1.180 \mathrm{~m}^{3} / \mathrm{s}\right) \text { (engine running at } 102 \mathrm{Nm}\right)\end{array}$ \\
\hline 139 to 143 & $\begin{array}{l}\text { PPMD measurement, single dilution with dilution ratios: 20, 12, 10, } 7 \text {, and } 5 \text { (engine } \\
\text { running at } 102 \mathrm{Nm} \text { ) }\end{array}$ \\
\hline 144 to 148 & $\begin{array}{l}\text { PPMD measurement, single dilution with dilution ratios: } 20,13,10,8 \text {, and } 5 \text { (engine } \\
\text { running at } 122 \mathrm{Nm} \text { ) }\end{array}$ \\
\hline 149 to 151 & $\begin{array}{l}\text { PPMD measurement, dual dilution with dilution ratios: } 80,100 \text { and } 180 \text { (engine running } \\
\text { at } 102 \mathrm{Nm} \text { ) }\end{array}$ \\
\hline 152 to 155 & $\begin{array}{l}\text { PPMD measurement, dual dilution with dilution ratios: 70, 120, 150, and } 300 \text { (engine } \\
\text { running at } 102 \mathrm{Nm} \text { ) }\end{array}$ \\
\hline 156 to 164 & $\begin{array}{l}\text { Post-tailpipe centerline measurement, corresponding distances and dilution ratios are: } \\
3.81 \mathrm{~cm}, \mathrm{dr} 1 ; 15.24 \mathrm{~cm}, \mathrm{dr} 2 ; 27.94 \mathrm{~cm}, \mathrm{dr} 3.2 ; 38.1 \mathrm{~cm}, \mathrm{dr} 4.2 ; 60.96 \mathrm{~cm}, \mathrm{dr} 6.5 ; 73.66 \mathrm{~cm} \text {, } \\
\mathrm{dr} 7.9 ; 106.68 \mathrm{~cm}, \mathrm{dr} 13.8 ; 116.84 \mathrm{~cm}, \mathrm{dr} 15.7 ; 152.4 \mathrm{~cm}, \mathrm{dr} 26.2 \\
\text { (engine running at } 122 \mathrm{Nm} \text { ) }\end{array}$ \\
\hline 165 to 175 & $\begin{array}{l}\text { Post-tailpipe centerline measurement, corresponding distances and dilution ratios are: } \\
3.81 \mathrm{~cm}, \mathrm{dr} 1 ; 8.89 \mathrm{~cm}, \mathrm{dr} 1.5 ; 16.51 \mathrm{~cm}, \mathrm{dr} 2.8 ; 20.32 \mathrm{~cm}, \mathrm{dr} 3.4 ; 27.94 \mathrm{~cm}, \mathrm{dr} 4.2 ; 35.56 \\
\mathrm{~cm}, \mathrm{dr} 4.8 ; 63.5 \mathrm{~cm}, \mathrm{dr} 7.6 ; 78.74 \mathrm{~cm}, \mathrm{dr} 9.9 ; 91.44 ; \mathrm{m}, \mathrm{dr} 11.9 ; 109.22 \mathrm{~cm}, \mathrm{dr} 15.3 ; 152.4 \\
\mathrm{~cm}, \mathrm{dr} 31.8 \text { (engine running at } 102 \mathrm{Nm})\end{array}$ \\
\hline
\end{tabular}




\section{A.1 UNCERTAINTY ANALYSIS}

\section{A.1.1 Introduction}

Since all measurements in physical world are not "perfect." In general, a result of a measurement is the estimate of the true value of the measurand. For this reason, a statement of uncertainty for a measurement is necessarily to complete the information provided by the measurement estimation [177]. The term "uncertainty" is formally defined as "parameter, associated with the result of a measurement that characterizes the dispersion of the values that could reasonably be attributed to the measurand [177]" in International Organization for Standardization (ISO) "Guide to the Expression of Uncertainty in Measurement (GUM)." There are varied possible sources of uncertainty in a measurement including "a) incomplete definition of the measurand; b) imperfect realization of the definition of the measurand; c) non-representative sampling - the sample measured may not represent the defined measurand; d) inadequate knowledge of the effects of environmental conditions on the measurement or imperfect measurement of environmental conditions; e) personal bias in reading analogue instruments; f) finite instrument resolution or discrimination threshold; g) inexact values of measurement standards and reference materials; h) inexact values of constants and other parameters obtained from external sources and used in the data-reduction algorithm; i) approximations and assumptions incorporated in the measurement method and procedure; j) variations in repeated observations of the measurand under apparently identical conditions.[177]" Those sources of uncertainty may be independent or may contribute to each other. This chapter focused on evaluating the combined standard uncertainties of certain measurands as described in later sections of this chapter. The procedures of evaluating combined uncertainties followed the steps defined in the abovementioned ISO GUM document along with reasonable simplifications.

In most cases a measurement $\mathrm{Y}$ is not directly measured but is calculated indirectly. Assuming $Y$ is determined by quantities $X_{1}, X_{2}, \ldots, X_{N}$ with relationship $f$ :

$$
Y=f\left(X_{1}, X_{2}, \ldots, X_{N}\right)
$$

Equation A1. 1 
The input quantities $X 1, X 2, \ldots, X N$ may be directly measured or themselves depend on other quantities. The values and uncertainties of $X 1, X 2, \ldots, X N$ may be determined from various sources such as a single or repeated observations, or experience, or handbook reference values. The relationship $f$ may be written explicitly or may be determined experimentally. When the relationship $\mathrm{f}$ is explicit and the combined standard uncertainty of $Y$ is then evaluated from

$$
u(Y)=\sqrt{\sum_{i=1}^{N} \sum_{j=1}^{N} \frac{\delta Y}{\delta X_{i}} \frac{\delta Y}{X_{j}} u\left(X_{i}, X_{j}\right)}
$$

Equation A1. 2

where $\partial Y / \partial X_{i}$ are referred to as sensitivity coefficients and $u\left(X_{i}, X_{j}\right)$ is the covariance of $X_{i}$ and $X_{j} \cdot u\left(X_{i}, X_{j}\right)$ becomes the variance of $X_{i} u\left(X_{i}\right)^{2}$ when $i$ equals to $j$.

\section{A.1.2 Uncertainty Calculation}

\section{A.1.2.1 Exhaust Flow Rate}

The calculation of exhaust flow was conducted by adding the fuel mass consumption rate (converted to standard condition volumetric flow rate SCFM) with the intake flow rate measured by the LFE. The analysis of fuel consumption rate measurement was performed by check the weight different of the fuel tank over a period of time for each steady state test condition:

$$
\dot{m}=\frac{w_{2}-w_{1}}{\Delta T}
$$

Equation A1. 3

where $\dot{m}$ was the fuel consumption rate, $w_{2}$ and $w_{1}$ were fuel tank weights read from the digital scale display over a period time $\Delta T$ for a steady state test. The uncertainty of the weight reading from the digital scale was $0.05 \mathrm{lbs}$. Since two different weight reading were independent, analysis of combined standard deviation of fuel consumption rate could be shown as:

$$
u(\dot{m})=\sqrt{\left[\frac{\delta \dot{m}}{\delta w_{1}} u\left(w_{1}\right)\right]^{2}+\left[\frac{\delta \dot{m}}{\delta w_{2}} u\left(w_{2}\right)\right]^{2}}=\frac{\sqrt{u\left(w_{1}\right)^{2}+u\left(w_{2}\right)^{2}}}{\Delta T}=\frac{\sqrt{2} \times 0.05 l b s}{\Delta T} \text { Equation A1. } 4
$$


The analysis of fuel consumption rate indicated that the uncertainty of measured fuel consumption rate could be large reduced by extending the test period and was irrelevant to the pre-test and post-test weight readings. Table 10 shows the fuel consumption rate measurement data with analyzed uncertainties.

Table 10 Fuel consumption rate and uncertainty of each steady state

\begin{tabular}{|c|c|c|c|c|}
\hline $\begin{array}{c}\text { Engine toque } \\
(\mathrm{Nm})\end{array}$ & $\begin{array}{c}\text { Fuel consumption over } \Delta T \\
(\mathrm{lbs})\end{array}$ & $\begin{array}{c}\text { Time } \Delta T \\
(\mathrm{~s})\end{array}$ & $\begin{array}{c}\text { Converted flow rate } \\
(\mathrm{SCFM})\end{array}$ & $\begin{array}{c}\text { Uncertainty } \\
(\mathrm{SCFM})\end{array}$ \\
\hline 122 & $\mathrm{~A} 1.45$ & 1761 & 2.92 & 0.03 \\
\hline 102 & 4.05 & 1490 & 2.17 & 0.04 \\
\hline 68 & 4.20 & 2020 & 1.66 & 0.03 \\
\hline 34 & 1.80 & 1395 & 1.03 & 0.04 \\
\hline 0 & 0.90 & 1080 & 0.67 & 0.05 \\
\hline
\end{tabular}

The intake air flow rate was measured with a Meriam LFE model 50MC2-2. The LFE was calibrated by the manufacturer with a calibration curve provided. The accuracy level of the calibration was $\pm 0.86 \%$ of actual reading and the calibration was traceable to the National Institute of Standards and Technology according the manufacturer's statement. However, a combined standard deviation analysis for measured LFE flow rate was performed based on the test configuration of this research using equations 5.5, 5.6, and 5.7 of section 5.1.1. The final uncertainty level was lower than the LFE manufacturer's uncertainty level. It was achieved with the use of an ultra-low variable capacitance pressure transducer for differential pressure measurement. The equation to calculate the LFE flow rate could be expressed in general form as:

$$
\operatorname{Flow}(S C F M)=f(D P, A P, T, H)
$$

Equation A1. 5

where $D P$ was the differential pressure of the LFE in $i_{n} H_{2} O, A P$ and $T$ were the upstream absolute pressure in $\mathrm{InHg}$ and temperature in $\mathrm{K}$ of LFE, $H$ was the measured relative humidity from the humidity sensor in percentage. Details of those input quantities with uncertainty levels are shown in Table 11. 
Table 11 Sensor specifications for LFE flow rate measurement

\begin{tabular}{|l|l|l|l|l|}
\hline Sensor & $\begin{array}{l}\text { Differential } \\
\text { Pressure }\end{array}$ & $\begin{array}{l}\text { Absolute } \\
\text { Pressure }\end{array}$ & Temperature & Humidity \\
\hline $\begin{array}{l}\text { Manufacture } \\
\& \text { Model }\end{array}$ & ASHCROFT & OMEGA & Omega & Honeywell \\
Number & & $\begin{array}{l}\text { IXL303- } 10^{\prime \prime} \mathrm{WC} \\
015 \mathrm{~A} 10 \mathrm{~V}\end{array}$ & $\mathrm{~K}-$ Thermocouple & HIH-4000-001 \\
\hline $\begin{array}{l}\text { Accuracy } \\
\text { level }\end{array}$ & \pm 0.025 & \pm 0.076 & Greater of $2.2^{\circ} \mathrm{C}$ or & \pm 3.5 \\
\hline
\end{tabular}

The uncertainty of intake air flow rate was expressed as:

$$
u(f)=\sqrt{\left[\frac{\delta f}{\delta D P} u(D P)\right]^{2}+\left[\frac{\delta f}{\delta A P} u(A P)\right]^{2}+\left[\frac{\delta f}{\delta T} u(T)\right]^{2}+\left[\frac{\delta f}{\delta H} u(H)\right]^{2}} \quad \text { Equation A1. } 6
$$

The calculation of uncertainty analysis was performed in mathematical software Matlab with symbolic toolbox. Following outputs for the expressions of sensitivity coefficients were obtained from the Matlab symbolic equation analysis outputs.

Since this research was conducted on a natural aspirated diesel engine with a constant engine speed, it had been observed the measured intake air flow was maintained at the range from around $60 \mathrm{SCFM}$ to $64.5 \mathrm{SCFM}$ with varied engine torque settings. For a typical measured intake flow rate with sensor signals $\mathrm{DP}=5.0 \mathrm{inH}_{2} \mathrm{O}, \mathrm{AP}=29.00 \mathrm{inHg}$, $\mathrm{T}=293 \mathrm{~K}$ and $\mathrm{H}=25 \%$, corresponding sensitivity coefficient values and variance multiplied value were:

$$
\begin{array}{lll}
\frac{\delta f}{\delta D P}=12.34 & \frac{\delta f}{\delta D P} u(D P)=0.309 & \frac{\delta f}{\delta A P}=2.13 \\
\frac{\delta f}{\delta A P} u(A P)=0.162 & \frac{\delta f}{\delta T}=-0.38 & \frac{\delta f}{\delta T} u(T)=-0.057 \\
\frac{\delta f}{\delta H}=-0.0057 & \frac{\delta f}{\delta H} u(H)=-0.020 &
\end{array}
$$


The measured intake flow rate with uncertainty was expressed as $61.85 \pm 0.35$ SCFM. The sensitivity coefficients analyzed above showed that the measured intake flow rate was most sensitive to differential pressure sensor's accuracy, whereas high accuracy was not required for the humidity sensor. An ASHCROFT IXLdp 10" WC ultra-low variable capacitance differential pressure transducer with $0.25 \%$ accuracy level was selected for this research to most effectively increase the intake air flow measurement accuracy. With combination of other sensors, low uncertainty levels of intake air flow rate measurement than the manufacturer's accuracy specifications were achieved in this research (for example, $61.85 \pm 0.35$ compared to specification value $61.85 \pm 0.53$ ). The recorded intake air flow rate values with measurement uncertainties over two days' measurement are shown in Figure 117.

Day one had the ambient air pressure $28.89 \mathrm{inHg}$ and ambient air temperature 294.3K, whereas day two had $29.35 \mathrm{inHg}$ and $303.4 \mathrm{~K}$, respectively (both days' relative humidity was around $25 \%$ ). The uncertainties of measured intake air flow rates of two different days shown in Figure 117 clearly illustrated the magnitude of LFE measurement uncertainty and the day to day influence on measurement uncertainty. For the uncertainty level of the calculated exhaust flow rate, it could be written as:

$$
u(\text { exhaust_flowrate })=\sqrt{u^{2}(\text { LFE_flow })+u^{2}(\text { fuel_flow })}
$$

While the extreme case of maximum contribution of fuel consumption rate converted flow rate uncertainty to the total exhaust flow rate uncertain was examined (when $u\left(L F E_{-}\right.$flow $)=0.342$ SCFM and $u($ fuel_flow $)=0.05$ SCFM), the result showed that the fuel consumption rate converted flow rate uncertainty only counted $1 \%$ to the total exhaust flow rate uncertainty. It was reasonable from above analysis that the LFE measured flow rate uncertainty was representative for the total exhaust flow rate uncertainty. To simplify the calculation of the primary dilution ratio uncertainties, a conservative constant value of \pm 0.36 SCFM was applied for the exhaust flow rate uncertainty. Further analysis of collected data gave the raw exhaust flow rate as $64.39 \pm$ 
0.36 SCFM and $64.64 \pm 0.36$ SCFM for engine running at 102 Nm @ $1800 \mathrm{rpm}$ and 122 Nm@1800 rpm, respectively two running conditions tested for the tunnel measurement.

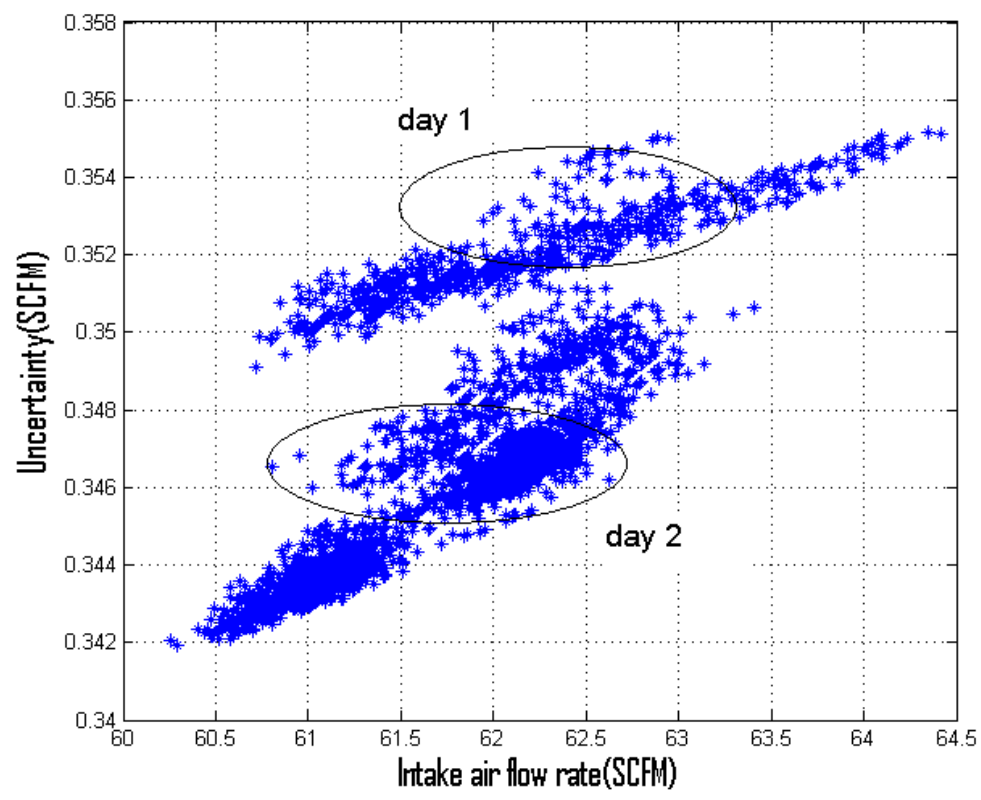

Figure 117 Intake air flow rate and uncertainties over two days of measurement

\section{A.1.2.2 Dilution Ratios}

Dilution ratios of the exhaust flow were calculated in two different ways for the tunnel test and tailpipe test, respectively, as described in section 5.1.2. The uncertainty of measured dilution ratio was the major source of uncertainties for total particle number and particle matter mass calculations when the measured particle size distributions were converted back to the stack, which was done by multiplying the particle size distribution curves by the dilution ratios. According to equation 5.9, the combined uncertainty level of dilution ratio for the tailpipe test was written as:

$$
u\left(D R_{\text {tailpipe }}\right)=\sqrt{\frac{1}{N O x_{-}^{2} m} u^{2}\left(N O x_{-} e\right)+\frac{N O x^{2} e_{-}}{N O x_{-}^{4} m} u^{2}\left(N O x_{-} m\right)} \quad \text { Equation A1. } 8
$$

The CLA-720MA $\mathrm{NO}_{\mathrm{x}}$ analyzer was calibrated with a span gas of 505ppm $\mathrm{NO}_{\mathrm{x}}$ concentration level. This analyzer was calibrated to have a linearity of slope coefficient within (0.99 1.01) and it was zero and span calibrated frequently during test intervals 
(within 30 minutes). The accuracy level of the $\mathrm{NO}_{\mathrm{x}}$ analyzer was regarded to be $\pm 1 \%$ of reading. The uncertainty equation became:

$$
u\left(D R_{\text {tailpipe }}\right)=\sqrt{\frac{N O x^{2} \_}{N O x^{2}{ }_{-}}(0.01)^{2}+\frac{N O x^{2} \_}{N O x^{2} \_m}(0.01)^{2}}=0.014 D R_{\text {tailpipe }}
$$

The uncertainty of dilution ratio calculated by the method of dividing the raw exhaust $\mathrm{NO}_{\mathrm{x}}$ concentration by measured $\mathrm{NO}_{\mathrm{x}}$ concentration was proportional to the calculated dilution ratio.

The calculation of dilution ratios in side the primary dilution tunnel can be expressed as followed equation by combining equations 5.9, 5.10 and 5.11:

$$
D R_{\text {primary }}=f\left(N O x_{-} s, N O x_{-} m, Q_{s s v} Q_{e x}\right)=\frac{N O x_{-} s \times Q_{s s v}}{N O x_{-} m \times Q_{e x}}
$$

The uncertainty level of the primary flow rate was considered to be $\pm 2 \%$ of reading according to the CFR Part 1065 specifications on the SSV flow rate calibration and linearity requirements. The combined uncertainty level equation was derived as:

$$
u\left(D R_{\text {primary }}\right)=0.017 \frac{N O x_{-} s \times Q_{s s v}}{N O x_{-} m \times Q_{e x}}+0.36 \frac{N O x_{-} s \times Q_{s s v}}{N O x_{-} m \times Q_{e x}^{2}}
$$

The measurement uncertainty of measured $\mathrm{NO}_{\mathrm{x}}$ concentration levels and primary flow rate contributed to the first term of the result uncertainty equation A1.11 for the primary dilution ratio. This term was similar to equation A1.9 as the uncertainty of this fraction being proportional to the calculated dilution ratio. The secondary term was the uncertainty level caused by the exhaust flow rate measurement uncertainty. Since only two raw exhaust flow rates with close values were involved during the tunnel test research, the flow rate value of 64.39 SCFM was used to replace one $Q_{e x}$ of the secondary term in the uncertainty equation, the final uncertainty level of the primary dilution ratio can be rewritten as 


$$
u\left(D R_{\text {primary }}\right)=0.0226 D R_{\text {primary }}
$$

With described conservatively simplification, the uncertainty levels of both tailpipe and primary tunnel dilution ratios were expressed as be linearly proportional to the calculated dilution ratios, which provided analyze of uncertainties related to particle size distributions back to stack conversion convenient with reasonable precision. The uncertainties of dilution ratios of the tailpipe exhaust and the primary tunnel can be plotted as Figure 118:

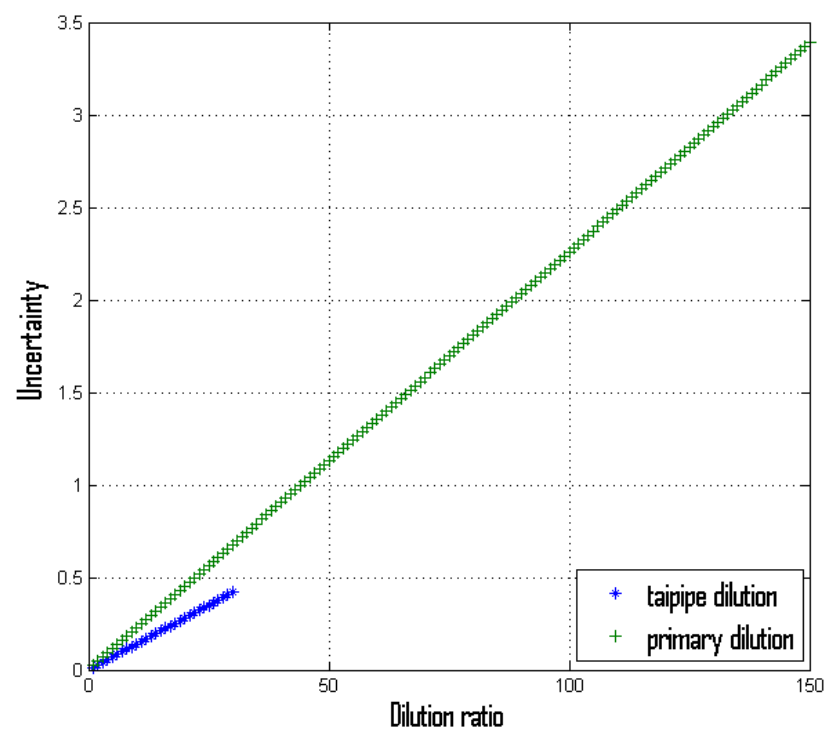

Figure 118 Dilution ratios and measurement uncertainty

The uncertainty level of primary dilution ratio of same value was higher than that of the tailpipe dilution ratio. It could be explained by having more input quantities for the primary dilution ratio equation than that of the tailpipe dilution ratio calculation. Since the tailpipe tested locations with dilution ratios up to 30 , uncertainty levels for dilution ratios beyond that value was not shown in Figure 118.

Equation 5.12 in section 5.1.2 gives the equation for calculation of secondary dilution ratios. A general form of equation 5.12 was written as:

$$
D R_{2 n d}=f\left(Q_{\text {vacuum }}, Q_{d m s}, Q_{\text {air }}\right)
$$


The vacuum pump flow rate $Q_{\text {vacuum }}$ and the secondary dilution air flow rate $Q_{\text {air }}$ were measured with two Smart Trak® 2 Model 100 mass flow controller / meters with accuracy level of $\pm 0.7 \%$ of reading. The DMS500 was set to run with a constant sample flow rate of $0.248 \pm 0.005$ SCFM which was maintained by an Aalborg mass flow controller inside the DMS500. The combined standard uncertainty level for the secondary dilution ratio was then written as:

$$
u\left(D R_{2 n d}\right)=\frac{\sqrt{0.007^{2}\left(Q_{\text {vacuum }} Q_{a}\right)^{2}+0.005^{2} Q_{a}^{2}+0.007^{2}\left(Q_{\text {vacum }}+Q_{d m s}\right)^{2} Q_{a}^{2}}}{\left(Q_{\text {vacuum }}+Q_{\text {dms }}-Q_{\text {air }}\right)^{2}} \text { Equation A1.14 }
$$

The vacuum pump flow rates were set to 2.00 SCFM and 2.25 SCFM during the secondary tunnel test. The secondary dilution ratios and their uncertainty levels are shown in Figure 119. The secondary dilution ratios and their uncertainty levels were used to convert the particle size distribution measurements to the secondary tunnel inlet in the primary tunnel and to the engine stack. Further observations on the uncertainty curves in Figure 119 introduced a simplified analytical expression of the secondary dilution ratio uncertainty with adequate accuracy. The final equation for the secondary dilution ratio uncertainty was obtained by a second order regression with $R^{2}=1$ and was written as:

$$
u\left(D R_{2 n d}\right)=0.0096 D R_{2 n d}^{2}-0.0096 D R_{2 n d}
$$

Equation A1. 15

This simplified uncertainty level equation gave less than $1 \%$ error compared to values in Figure 119 and such an equation largely simplified uncertainty analysis of the total secondary dilution ratio, which was written as:

$$
D R_{2 n d_{-} \text {total }}=D R_{\text {primary }} D r_{2 n d}
$$

Equation A1. 16

The combined uncertainty of total secondary dilution ratio was then:

$$
u\left(D R_{2 n d_{-} \text {total }}\right)=D R_{2 n d_{-} \text {total }} \sqrt{0.0226^{2}+\left(0.0096 D R_{2 n d}-0.0096\right)^{2}}
$$




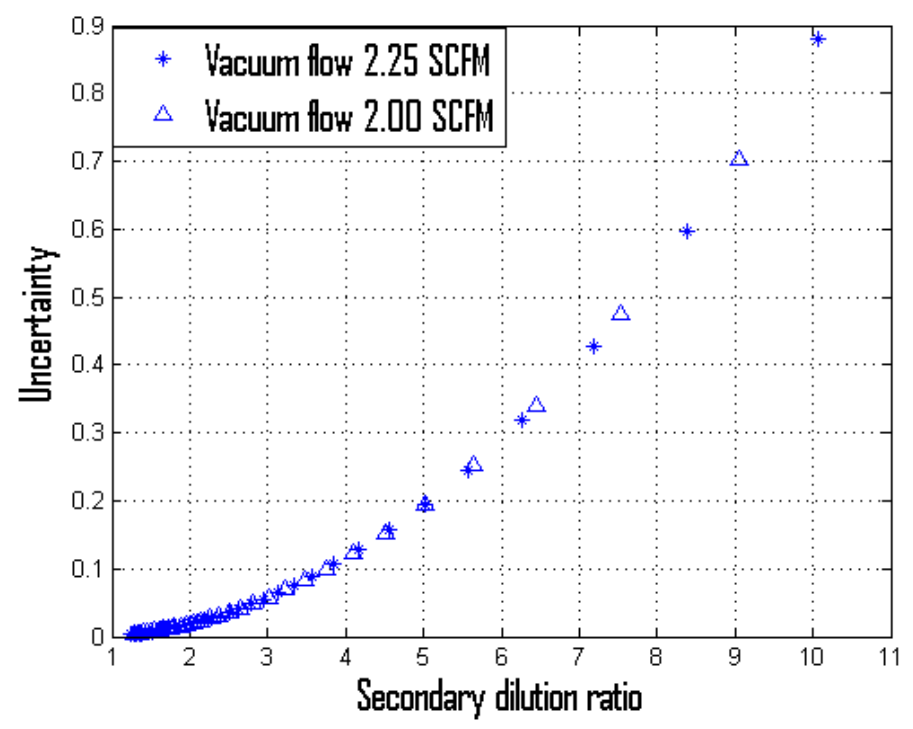

Figure 119 Secondary dilution ratios and corresponding uncertainty levels

Further analysis of equation A1.17 indicated when the secondary dilution ratio was less than 5 (including 5), the uncertainty level of the primary dilution ratio contributed major part to the total secondary dilution ratio uncertainty. For secondary dilution ratio greater than 5, the uncertainty level of the secondary dilution ratio contributed major part to the total secondary dilution ratio uncertainty. For a primary dilution ratio of 12.5 and secondary dilution ratio range from 1 to 10 , the total secondary dilution ratios and corresponding uncertainty levels can be plotted as Figure 120:

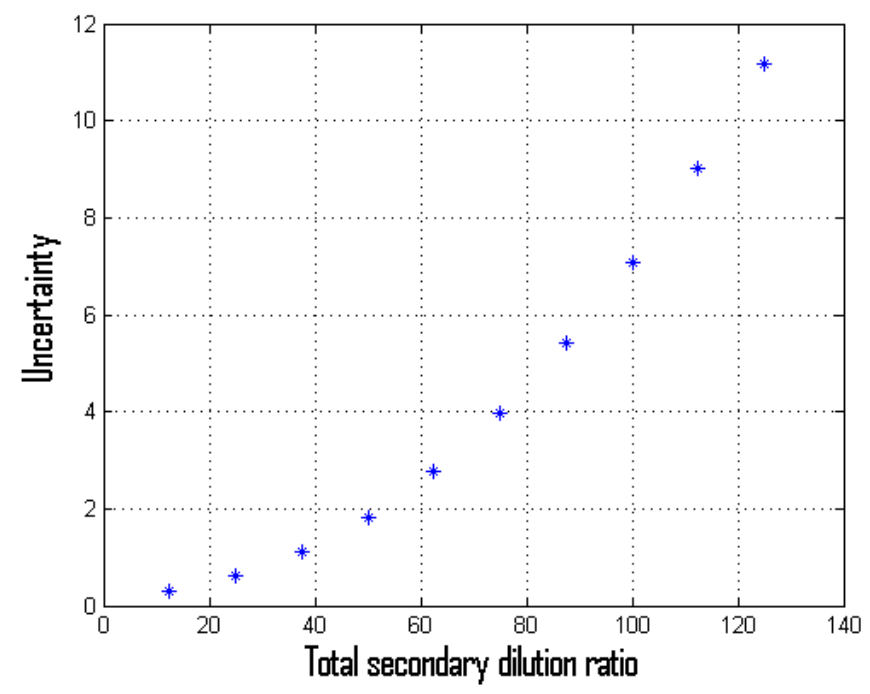

Figure 120 Total secondary dilution ratios and corresponding uncertainty levels 
The flow rates measured by the PPMD had uncertainty levels of $1 \%$ of reading. The uncertainty analysis for the dilution ratios of the PPMD was more straightforward than tunnel test and tailpipe test. Since the dilution ratios were calculated by dividing the total flow rate by the sample flow rate and both flow rates had uncertainty level of $1 \%$ of reading, the uncertainty level of the dilution ratio of the PPMD is:

$$
u\left(D R_{p p m d}\right)= \begin{cases}0.014 D R_{p p m d} & (\text { single dilution }) \\ 0.020 D R_{p p m d} & (\text { double dilution })\end{cases}
$$

\section{A.1.2.3 Particle Size Distribution}

The DMS500 fast particulate spectrometer was used to obtain the Diesel particle size distributions. This instrument was calibrated by the manufacturer Cambustion for particle size and number. Nucleated liquid sulfuric acid, atomised and dried solid sodium chloride aerosols were used with a TSI 3080 electrostatic classifier and a TSI 3081 DMA to perform DMS500 calibration for particle size less than $100 \mathrm{~nm}$. NIST traceable polystyrene latex (PSL) sphere of certified size were used to for calibration for particle size over $100 \mathrm{~nm}$. The DMS manufacturer certified that the size measured with DMS500 was within $5 \%$ of the standard reference in the region of 5-300 nm and within $10 \%$ of the standard for diameters $>300 \mathrm{~nm}$, and the number concentration measured with DMS500 was within $10 \%$ of the standard reference value. Since only sub-100nm particles were observed in this research, the particle number concentration uncertainty level measured by DMS500 in this study was considered to be $\pm 10 \%$ of measured values. The instrument sensitivity to noise was treated as negligible because the highest absolute noise level for measured size range was at the magnitude of $10^{3} \mathrm{dD} / \mathrm{d} \operatorname{logDP} / \mathrm{cc}$ and the minimum $\mathrm{dD} / \mathrm{d} \log \mathrm{DP} / \mathrm{cc}$ value measured was at the magnitude of $10^{7} \mathrm{dD} / \mathrm{d} \log \mathrm{DP} / \mathrm{cc}$.

Equation 5.18 was used to integrate over the DMS500 spectra data and establish the correlation between the particle size distribution and particle mass concentration. According to the application note issued by Cambustion [178], the integrated PM mass

concentration level has a uncertainty level of $\pm 1 \%$ of calculated value in $\mu \mathrm{g} / \mathrm{m}^{3}+2$ 
$\mu \mathrm{g} / \mathrm{m}^{3}$. When the total PM mass concentration level was corrected back to engine stack, this calculated value was multiplied by dilution ratio:

$$
M_{\text {stack }}=(M+2) \times D R
$$

Equation A1. 19

where $M_{\text {stack }}$ was the stack corrected PM mass concentration level, $M$ was the measured mass concentration level and $D R$ was the measured dilution ratio, which could be posttailpipe dilution ratio, PPMD dilution ratio, or tunnel dilution ratio. The uncertainty level of $M_{\text {stack }}$ was expressed as $\pm u\left(M_{\text {stack }}\right)$ where $u\left(M_{\text {stack }}\right)$ was:

$$
u\left(M_{\text {stack }}\right)=\sqrt{D R^{2} \times(0.01 M)^{2}+M^{2} u^{2}(D R)}
$$

The values of $u(D R)$ varied when the dilution ratio was measure at different locations and were expressed as equation A1.9, A1.12, A1.15, and A1.17.

When the total particle number concentration level was examined, it was obtained by adding all measured $38 \mathrm{dD} / \mathrm{d} \log D P$ values in 38 size range and multiplying the summation by the range interval 0.0625 .

$$
N_{\text {total }}=0.0625 \sum_{i=1}^{38} N_{i}
$$

Equation A1. 21

The uncertainty level of calculated $N_{\text {total }}$ was then:

$$
u\left(N_{\text {total }}\right)=0.00625 \sqrt{\sum_{i=1}^{38} N^{2}}
$$

Similar to the stack corrected mass concentration uncertainty, the stack corrected total particle number concentration was:

$$
U\left(N_{\text {total_stack }}\right)=\sqrt{D R^{2} u^{2}\left(N_{\text {total }}\right)+N_{\text {total }}^{2} u^{2}(D R)}
$$

The expression of $u(D R)$ also varies with different sample locations as in equation A1.19. 